\title{
Virtual worlds, real healing : the use of virtual reality for assessment and treatment of stress and anxiety
}

Citation for published version (APA):

Gorini, A. (2010). Virtual worlds, real healing : the use of virtual reality for assessment and treatment of stress and anxiety. [Doctoral Thesis, Maastricht University]. Maastricht University. https://doi.org/10.26481/dis.20100922ag

Document status and date:

Published: 01/01/2010

DOI:

10.26481/dis.20100922ag

Document Version:

Publisher's PDF, also known as Version of record

\section{Please check the document version of this publication:}

- A submitted manuscript is the version of the article upon submission and before peer-review. There can be important differences between the submitted version and the official published version of record.

People interested in the research are advised to contact the author for the final version of the publication, or visit the DOI to the publisher's website.

- The final author version and the galley proof are versions of the publication after peer review.

- The final published version features the final layout of the paper including the volume, issue and page numbers.

Link to publication

\footnotetext{
General rights rights.

- You may freely distribute the URL identifying the publication in the public portal. please follow below link for the End User Agreement:

www.umlib.nl/taverne-license

Take down policy

If you believe that this document breaches copyright please contact us at:

repository@maastrichtuniversity.nl

providing details and we will investigate your claim.
}

Copyright and moral rights for the publications made accessible in the public portal are retained by the authors and/or other copyright owners and it is a condition of accessing publications that users recognise and abide by the legal requirements associated with these

- Users may download and print one copy of any publication from the public portal for the purpose of private study or research.

- You may not further distribute the material or use it for any profit-making activity or commercial gain

If the publication is distributed under the terms of Article $25 \mathrm{fa}$ of the Dutch Copyright Act, indicated by the "Taverne" license above, 



\section{Virtual Worlds, Real Healing}

The Use of Virtual Reality for Assessment and Treatment of Stress and Anxiety

Alessandra Gorini 
Printed by: Tipografia Milanese

(C) Alessandra Gorini, Milano 2010

Cover:

Hieronymus Bosch. The Extraction of the Stone of Madness (1475-1480). Museo del Prado, Madrid, Spain.

The Gothic inscription reads:

Meester snijt die keye ras

mijne name is Lubbert Das

(Master, cut away the stone

my name is Lubbert Das)

Many years have gone since doctors wearing funnel hats tried to extract the stone of madness from the head of their patients, and nowadays the funnel hats have been replaced by head mounted display...but other many years will pass before we can fully understand the secrets of mind. 


\section{Virtual Worlds, Real Healing}

The Use of Virtual Reality for Assessment and Treatment of Stress and Anxiety

\section{PROEFSCHRIFT}

ter verkrijging van de graad van doctor aan de Universiteit Maastricht, op gezag van de Rector Magnificus, Prof. mr. G.P.M.F. Mols volgens het besluit van het College van Decanen, in het openbaar te verdedigen

op woensdag 22 september 2010 om 12.00 uur

door

Alessandra Gorini 


\section{Promotor:}

Prof. Dr. E.J.L. Griez

\section{Co-promotor:}

Dr. K.R.J. Schruers

Beoordelingscommissie:

Prof. dr. A. Jansen

Dr. N. Nicolson

Prof. dr. F. Verhey

Prof. dr. J. Vlaeyen

Prof. dr. B.K. Wiederhold (Virtual Reality Medical Center, San Diego, USA) 
Almio Nonno Angelo, che aurebbe tanto voluto esserci... 

"Reality is merely an illusion, albeit a very persistent one" ALBERT EINSTEIN 



\section{TABLE OF CONTENTS}

II The role of immersion and narrative in mediated presence: the Virtual Hospital Experience

III Assessment of the emotional responses produced by exposure to real food, virtual food and photographs of food in patients affected by eating disorders

IV Non homogeneous results in place learning among panic disorder patients with agoraphobia

Intermezzo Virtual worlds, real healing on obese patients with emotional eating treatment to reduce anxiety in minor surgical operations: a randomized controlled study

VII Emotional response to virtual reality exposure across different cultures: the role of the attribution process virtual worlds in clinical psychology

IX Summary and concluding remarks

Appendix A Virtual Reality for the treatment of anxiety disorders

Summary

Nederlandse Samenvatting

Dankwoord 

CHAPTER I

General introduction 



\section{Virtual reality: from technology to presence}

Virtual Reality (VR) is more than a fancy technology: it is an advanced form of human-computer interface that allows users to interact with and become immersed in a computer-generated environment in a naturalistic way. Using visual, aural or haptic devices, the human operator can move and interact with the virtual world, experiencing the environment as if it were an extension of the real world.

From a technological point of view, VR is made possible by the capability of computers to synthesize a $3 \mathrm{D}$ graphical environment from numerical data. Different input devices sense the subjects' reactions and motions, while the computer modifies the environment accordingly, giving subjects the illusion of interacting with, and being immersed in it.

From a psychological point of view, VR can be considered an advanced imaginative system: an experiential form of imagery that is as effective as reality in inducing a wide range of cognitive and emotional responses. As discussed later in this chapter, this feature makes VR an innovative instrument to assess and treat a wide range of mental conditions.

\section{The VR technology}

A typical VR system includes the following components:

Hardware:

- the computational device: a desktop or a laptop pc equipped with an advanced image graphic card;

- different peripheral devices (visual, aural or haptic devices);

- a non immersive or immersive image display system: a screen or a head mounted display (HMD);

- a motion sensor (or tracking device), usually integrated in the HMD, that tells the computer where the user is looking at on the basis on his/her head movement.

Software:

- the VR application

According to the hardware and software included in a VR system it is possible to distinguish between different kinds of virtual settings:

- desktop VR: it uses a computer monitor as display to provide graphical interface for users. Desktop VR is cost-effective when 
compared to the immersive VR as it does not require any expensive hardware and software and is also relatively easy to develop;

- fully immersive $V R$ : it completely immerses the user inside the computer generated world, and can be achieved by using either the technologies of Head-Mounted Display (HMD) or multiple projections. Immersive VR with HMD uses it to project virtual images just in front of the eyes and allows users to focus on display without distraction. A magnetic sensor inside the HMD detects the users' head motion and feeds that information to the attached processor. When the user turns his or her head the displayed graphics reflects the changing viewpoint (Fig. 1);

- CAVE: a CAVE is a small room where a computer-generated world is projected on the walls. The projection is made on both front and sidewalls. This solution is particularly suitable for collective VR experiences because it allows different people to share the same experience at the same time;

- augmented reality: it is based on the combination of real and virtual stimuli. This system blurs the line between what is real and what is computer-generated by enhancing what users see, hear, feel and smell adding graphics, haptics and smells to the natural world as it exists. Compared to immersive VR that completely immerses a user inside a synthetic environment, augmented reality superimposes computer-generated information upon or composed with the real world. Therefore, AR supplements reality, rather than completely replacing it.

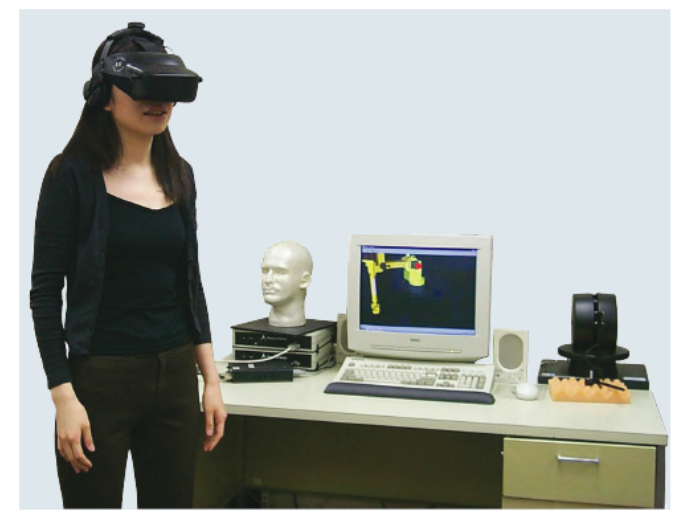

Fig. 1. A typical fully-immersive VR setting in which the user experiences the virtual environments wearing an HMD. 
The first commercial VR system was developed by Morton Heilig in $1956^{1}$ (Fig. 2), but the possibility to have a personalized virtual experience came only in 1968 when Ivan Sutherland developed the first HMD that allowed users to view 3D wire frame objects. In the early 1980s Krueger ${ }^{2}$, who was one of the first scientist to suggest a possible role of VR in the treatment of mental health disorders ${ }^{3}$, created the first program that allowed people to interact and change computer-generated images through their bodily movements. VR systems became available for the consumer market between 1985 and 1990 when several video games companies started to sell quite inexpensive HMD and data gloves to play and interact with virtual words.

Even if VR is still mainly known for games and simulation trainings industries, thanks to the incredible reduction of the hardware costs, in the last years its use in health care has become more and more widespread. Since the turn of the century VR healthcare applications have experienced a double-digit growth both worldwide and in the United States, and, according to a research report from Kalorama Information ${ }^{4}$ the 2010 US market for VR in surgery, medical education, therapy and other areas will grow to $\$ 290$ million. The growing interest in medical applications based on VR is also highlighted by the increasing number of scientific papers published each year on this topic: searching "virtual reality" as a keyword in Medline from 1995 to now, we found an average growth rate of nearly 15 per cent per year.

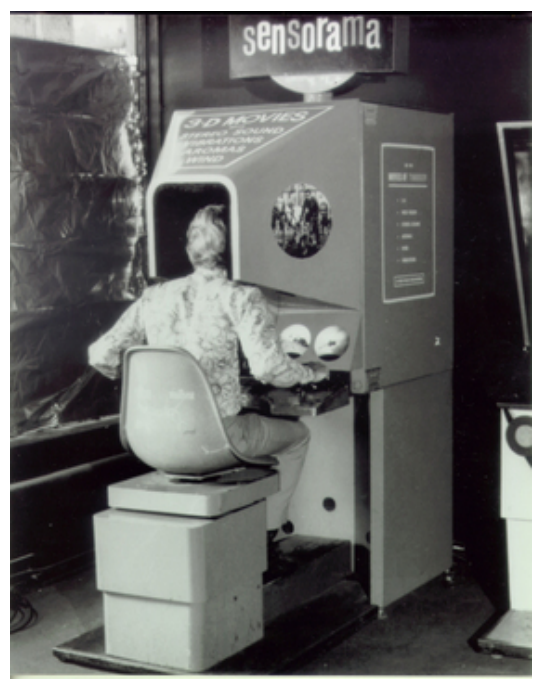

Fig. 2. The Morton Heilig, «Sensorama», 1962. 
Unfortunately, the huge advance in technology and the significant reduction of its cost does not apply to the VR software, that are still very expensive, not easily customizable by users, and usually based on not user-friendly interfaces that require continual maintenance and technical support. When used for clinical and therapeutic purposes, their weakness also include the limited possibility of tailoring the virtual environments to the specific requirements of the clinical or experimental setting, as well as the low availability of standardized protocols that can be shared by the community of researchers and clinicians.

A first significant attempt to address these challenges has been performed by Riva and coll. ${ }^{5}$ who have designed and developed NeuroVR (http://www.neurovr.org), a cost-free VR platform based on an open-source software, that allows non-expert users to easily modify a virtual environment and visualize it using either an immersive or non-immersive system. The NeuroVR Editor is realized through the customization of the User Interface of Blender, an integrated suite of 3D creation tools available on all major operating systems, under the GNU General Public License, that allows the free distribution of the complete source code of the program. Thanks to these features, clinicians and researchers around the world can run, copy, distribute, study, change and improve the NeuroVR software, to create the environments they need and to share them with the whole VR community (Fig. 3).

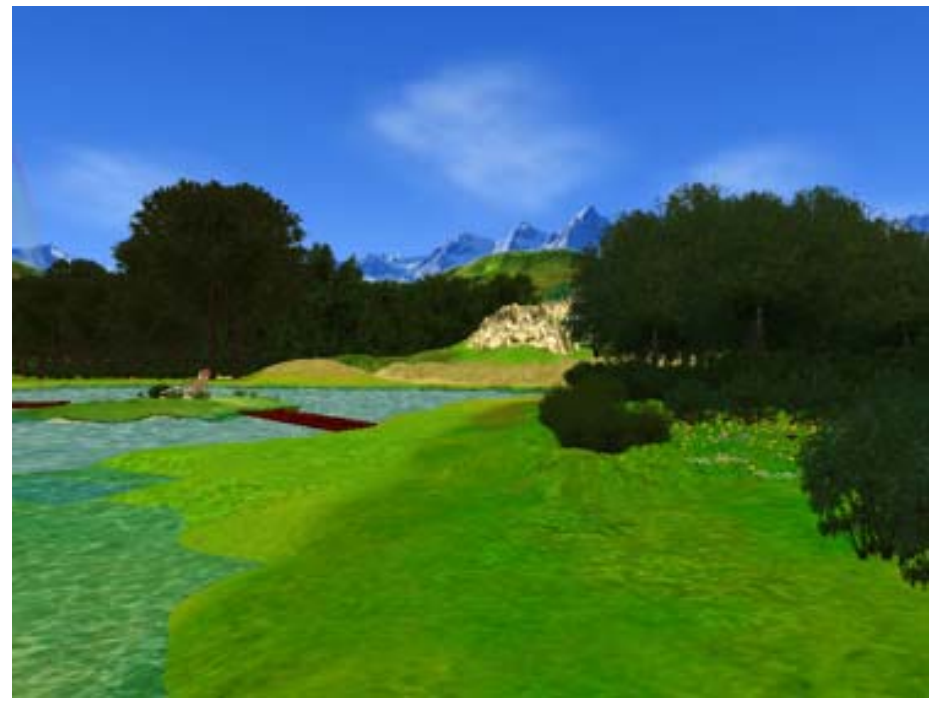

Fig. 3. A screenshot from NeuroVR. The Green Valley is one of the virtual environment included in this free, open-source software used to facilitate relaxation in anxious subjects. 
Finally, as we will discuss below, in the very last years, the diffusion of the Web 2.0 has further facilitated the creation of fully customizable virtual environments that can be used for many different purposes, including therapy.

\section{The VR experience}

As stated by Dourish ${ }^{6}$ VR is a technology that allows physical and virtual spaces to exist in parallel. But VR cannot be considered just as a pure technology ${ }^{7}$. VR is also an experiential interface that gives the users the impression of "being there", otherwise called "sense of presence" 8 , defined as a technology-induced illusion of being present in one (simulated) place when one is actually present in another (physical) place ${ }^{9}$. As shown by different researches, the level of presence is directly connected to the level of emotions and the quality of actions experienced in the virtual environment ${ }^{10}$.

Experiencing presence, VR users become not simply external observers of images provided by a computer screen, but active participants within a computer-generated 3D virtual world. This process is fundamental to make the subject able to transfer the knowledge acquired in the virtual environment to the real world. In a clinical perspective, after having identified an enriched environment that contains functional real-world demands, the technology is used to enhance the level of presence of the subject in the environment and to induce an optimal experience, that is defined as a positive, complex and rewarding state of consciousness ${ }^{11,12}$. Once subjects reache this optimal experience, they will be able to transfer this new knowledge in their behaviors, by linking the experience acquired in the virtual world to their actual experience in the real one. This is the key factor that makes VR a successful therapeutic instrument.

\section{Virtual reality in healthcare}

Although it is difficult to trace the origins of the application of VR to the treatment of mental health disorders, most people agree that Myrron Krueger was one of the first to propose it. In one of his books Krueger ${ }^{3}$ discussed the idea that patients may use an artificial experience in combination with a traditional psychotherapy to overcome the inhibitions usually present in real life. In particular, he suggested that, at least in some 
cases, patients are more comfortable relating to and interacting with computers, a finding that has been supported in a number of subsequent studies ${ }^{13-15}$ and particularly evident after the introduction of the on-line virtual worlds. Kruger can be also considered a pioneer in the use of VR for exposure therapy since he was the first to suggest that a virtual environment can be used to gradually introduce elements of change during a traditional therapeutic intervention.

On the basis of his observations, in the early to mid-1990s different clinicians started to use VR to treat patients. The virtual exposure therapy was immediately demonstrated to be effective in the treatment of specific phobias, such as acrophobia ${ }^{16}$, social phobia, and agoraphobia ${ }^{17}$. These studies pointed out that even though the VR environments were at that time primitive, the user could become immersed in the virtual world having a meaningful experience ${ }^{18}$. They also suggested that, in a virtual world, the therapist can easily accompany the patient to assist him/her in the desensitization process and to better understand how he/she processes and responds to information coming from specific threatening environments and situations. And most important, using virtual environments the therapist can control the speed and intensity of the therapeutic process, tailoring the virtual experience to each patient's specific needs.

Differently from what happens in other areas of medicine in which VR is mainly considered a simulation tool that offers the possibility to realize realistic body parts or entire avatars that interact with external devices such as surgical instruments as near as possible to their real models ${ }^{19,20}$, for clinical psychologists and psychiatrists the interaction focus of VR prevails on the simulation one: they use VR to provide a new human-computer interaction paradigm in which users are no longer simply external observers of images on a computer screen, but active participants within a computergenerated $3 \mathrm{D}$ virtual world ${ }^{21,22}$.

\section{Advantages of using VR in the treatment of anxiety disorders}

Virtual exposure therapy consists in exposing patients to virtual environments created ad hoc for specific disorders (for example, specific phobias) under the direct supervision of the therapist. The patient undergoes the therapeutic sessions in the therapist office where the virtual sessions substitute imaginative and in vivo exposure. 
Different meta-analyses ${ }^{23,24}$ have shown that psychological VR-based treatments are more effective than no treatment, but also slightly but significantly more effective than in vivo and imaginative exposure therapy. They also suggest that VR treatments have a statistically large effect on all affective domains and that these effects are significant ${ }^{25}$.

Compared to traditional exposure-based approaches, virtual therapy presents great advantages related to the ratio between costs and benefits. In a clinical setting costs refer not only to the expenditure in money and time, but also in emotional involvement requested to the patient. On the other side, benefits regard the effectiveness of the treatment, that can be defined as the achievement of the target set in the shortest time possible.

In particular, compared to in vivo and imaginative exposure, VR presents the following advantages:

- it guarantees the vividness of exposure: in the imaginative condition patients are trained to visualize the anxiety-provoking stimuli through mental images, but they often fail in doing that because they have difficulties in visualizing stressful scenes in a vivid way. VR provides a real-like experience that may be more emotionally engaging than imaginative exposure;

- it increases the controllability of experience: during in vivo exposure subjects experiences anxious situations and are exposed to phobic stimuli in semi-structured situations. The therapist cannot fully control the real-life events and the patient's reactions, with the high risk of provoking too much stress in the patient. On the contrary, VR gives the therapist the opportunity to recreate a structured and controlled hierarchy of real-like situations specific for the disorder that must be treated;

- during the virtual exposure, nothing the patients fear can "really" happen to them. With such assurance, they can freely explore, experiment, feel, live, and experience feelings and/or thoughts related to the anxious stimuli. Thus VR becomes a very useful intermediate step between the therapist's office and the real world;

- using the VR exposure the single fear components can be isolated more efficiently than in vivo exposure. For instance, treating the fear of flying, if landing is the most fearful part of the experience, it can be repeated as often as necessary without having to wait for the airplane to take-off several times.

Putting together, these observations highlight the effectiveness of VR exposure therapy in reducing costs and increasing benefits for both the 
therapist and the patient. In particular, the flexibility of the virtual environments allows the patient to be gradually exposed to critical stimuli, and to over practice in situations that are often much worse and more exaggerated than those that are likely to be encountered in real life. This allows patients to develop a sense of mastery and the confidence to carry out the task successfully.

Starting from 1995, different controlled and uncontrolled experimental studies have been conducted in order to investigate the effect of VR in the treatment of specific phobias (acrophobia, claustrophobia, fear of flying, fear of driving and spider phobia) and anxiety disorders (social phobia, panic disorders with agoraphobia and PTSD) ${ }^{5,26-30}$. A summary of these studies indicating the size of sample, the design, and their main clinical outcomes is reported in Appendix A ${ }^{30}$.

"Simulation technology has allowed clinicians to treat patients more effectively and efficiently, without concerns of excessive cost, loss of confidentiality and limited safety that arise with many conventional treatments," says Dr. Wiederhold, one of the pioneer in the use of VR to treat psychological problems. "The therapeutic benefits of using simulations are becoming increasingly well recognized and fully supported with results from controlled clinical trials" 31 .

\section{Outline of the present thesis}

The present thesis focuses on VR as a promising tool for assessment and treatment of stress and anxiety.

As we have seen in the previous paragraphs, in the last twenty years many researchers and clinicians have proposed to use VR to provide an innovative form of exposure therapy to patients suffering from different psychological disorders. The rationale behind the "virtual approach" is that real and virtual exposures elicit a comparable emotional reaction in subjects, even if, up to date, there are no experimental data that directly compare these two conditions.

The two following chapters (Chapter two and three) describe two experimental studies that investigate the theoretical premises of VR. In particular, the first one has been performed on a sample of healthy volunteers and regards the role played by immersion and context on the sense of presence. Knowing that the level of presence is a key feature for 
effective virtual experiences, this study is aimed to investigate which are the factors that can increase it.

On the other hand, in Chapter three we tested whether virtual stimuli are as effective as real ones, and eventually more effective than static photographs, in inducing anxiety in human subjects. To be sure that we were using really anxiety-provoking stimuli, we investigated the emotional reactions to real food, virtual foods, and photographs of food in two samples of patients affected, respectively, by anorexia and bulimia nervosa, compared to a group of healthy subjects. Even if preliminary, our results show that VR is more effective than photographs in eliciting emotional responses very similar to those expected in real life situations.

In a psychological perspective these findings support the use of VR to test the subjects' neuropsychological performance enhancing the ecological validity of the test and consequently increasing the validity of predictions about the patient's cognitive functioning in the real world. Chapter four shows how virtual environments can be effective to qualitative and quantitative assess neuropsychological deficits in a group of patients affected by panic disorder with agoraphobia, a psychological disorder whose etiology can be at least partially related to the presence of spatial orientation deficits that are difficult to be detected in real-life situations.

The second part of the thesis is dedicated to the use of VR to reduce clinical or situational anxiety in different medical conditions.

In particular, Chapter five investigates the use of a relaxing virtual environment to reduce stress and related emotional eating episodes in obese patients. Traditional psychological and behavioral interventions for the socalled "emotional eating disturbance" include stress management techniques, mainly based on imagination. Using a relaxing VR environment and a portable mp3 player, the aim of our study was to facilitate the relaxation training and to improve the perceived self-efficacy in eating control.

Still based on the hypothesis that immersion in a virtual environment providing specific visual and auditory relaxing stimuli can induce a significant reduction of stress and anxiety, in Chapter six and seven we described the use of VR as a distraction tool in patients who underwent minor surgical operations. In particular, Chapter seven presents a significant innovation in the study of VR effects investigating the role played by cultural and technological background of the users on their emotional responses. People from a small rural and isolated Mexican village characterized by a very traditional culture were exposed to a non-interactive, relaxing immersive environment during a surgical operation under local 
anesthesia while their level of pre-operative and operative anxiety was monitored.

Since to recent technological advances, many simulations can be now delivered over the Internet, as well as on hand-held portable devices (Fig. 4). This contributes to a wide healthcare dissemination and personalization in the areas of prevention, training, education, therapy, and rehabilitation. Chapter eight is focused on the role played by three-dimensional (3-D) online virtual worlds in eHealth applications, addressing some potential advantages and issues related to the use of the World Wide Web (WWW) in clinical practice. Due to the enormous diffusion of Web 2.0, telepsychology, and telehealth in general, have become accepted and validated methods for the treatment of many different health care concerns. This last chapter describes the development and implementation of a form of tailored immersive e-therapy called p-health (personalized health) whose key factor is interreality, that is the creation of a hybrid augmented experience merging physical and virtual worlds. Compared to conventional telehealth applications such as emails, chat, and videoconferences, the interaction between real and 3-D virtual worlds may convey greater feelings of presence, facilitate the clinical communication process, positively influence group processes and cohesiveness in group-based therapies, and foster higher levels of interpersonal trust between therapists and patients.

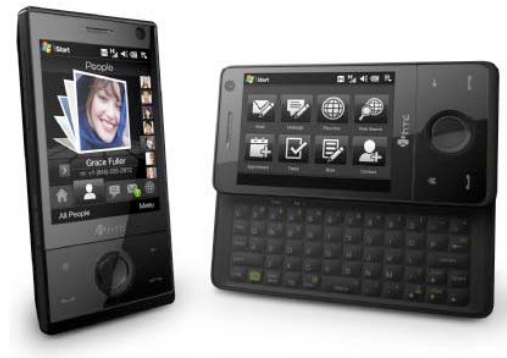

(a)

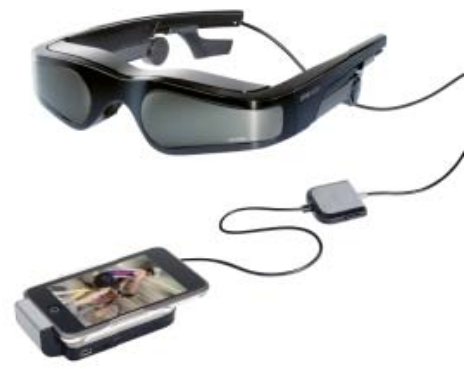

(b)

Fig. 4. (a) The HTC Touch Pro, one of the smartphone that can be used to provide mobile VR. (b) Some smartphones, like the Ipod, can be also connected to a HMD to increase the level of immersion of the user.

The use of on-line virtual worlds for therapeutic purposes is also discussed in the Intermezzo, a short letter testifying the growing interest of the entire scientific community for this innovative approach. 


\section{References}

1. Heilig M. (1962) Sensorama simulator.

2. Krueger M. (1983) Artificial Reality. Reading, MA: AddisonWesley.

3. Krueger M. (1991) Artificial Reality II. New York, NY: AddisonWesley.

4. Heffner S. (2007) Virtual reality in U.S. healthcare (markets for remote surgery, 3D modeling, pain distraction and other applications). Rockville, MD: Kalorama Information.

5. Riva G. Virtual reality in psychotherapy: review. Cyberpsychology \& Behavior 2005; 8(3):220-30.

6. Dourish P. (2001) Where the action is: The foundations of embodied interaction. Cambridge, MA: MIT Press.

7. Steuer J. Defining virtual reality: dimensions determining telepresence. Journal of Communication 1992; 42(4):73-93.

8. Riva G, Davide F, Ijsslsteijn WA (eds). Being there: Concepts, effects and measurements of user presence in synthetic environments. Amsterdam: IOS Press; 2003.

9. Waterworth JA, Waterworth EL, Mantovani F, Riva G. On feeling (the) present. Journal of Consciousness Studies 2010; 17(1-2).

10. Riva G, Mantovani F, Capideville CS, et al. Affective interactions using virtual reality: the link between presence and emotions. Cyberpsychol Behav 2007; Feb; 10(1):45-56.

11. Csikszentmihalyi M. (1975) Beyond Boredom and Anxiety. San Francisco: Jossey-Bass.

12. Csikszentmihalyi M. (1990) Flow: The psychology of optimal experience. New York: Harper Collins.

13. Joinson A. Social desirability, anonymity, and Internet-based questionnaires. Behav Res Methods Instrum Comput 1999; Aug; 31(3):433-8.

14. Joinson A. Self-disclosure in computer-mediated communication: the role of self awareness and visual anonimity. European Journal of Social Psychology 2001; 31(2):177-92.

15. Richman WL, Kiesler S, Weisband S, Drasgow F. A meta-analytic study of social desiderability distortionin computer-administered questionnaires, and interviews. Journal of Applied Psychology 1999; 84:754-75. 
16. Lamson RJ. (1997) Virtual therapy. Montreal, Canada: Polytechnic International Press.

17. Camara E. Virtual reality: applications in medicine and psychiatry. Hawaii Med J 1993; Dec; 52(12):332-3.

18. Bard SR. Virtual reality: an extension of perception. Noetic Sciences Review 1991; (Autumn):7-16.

19. Székely G, Satava RM. Virtual reality in medicine. British Medical Journal 1999; 319(7220):1305.

20. Satava RM, Jones SB. (2002) Medical applications of virtual reality. In: Stanney KM (ed). Handbook of Virtual Environments: Design, Implementation, and Applications. Mahwah, NJ: Lawrence Erlbaum Associates, Inc.

21. Riva G, Rizzo A, Alpini D. Virtual environments in the diagnosis, prevention, and intervention of age-related diseases: a review of VR scenarios proposed in the EC VETERAN project. Cyberpsychology \& Behavior 1999; 2(6):577-91.

22. Rizzo AA, Wiederhold B, Riva G, Van Der Zaag C. A bibliography of articles relevant to the application of virtual reality in the mental health field. Cyberpsychology \& Behavior 1998; 1(4):411-25.

23. Parsons TD, Rizzo AA. Affective outcomes of virtual reality exposure therapy for anxiety and specific phobias: a meta-analysis. J Behav Ther Exp Psychiatry 2008; Sep; 39(3):250-61.

24. Powers MB, Emmelkamp PM. Virtual reality exposure therapy for anxiety disorders: A meta-analysis. J Anxiety Disord 2008; 22(3):561-9.

25. Cohen J. A power primer. Psychological Bullettin 1992; 112:155-9.

26. Botella C, Quero S, Banos RM. Virtual reality and psychotherapy. Studies in Health Technology and Information 2004; 99:37-54.

27. Glantz K, Rizzo A, Graap K. Virtual reality for psychotherapy: current reality and future possibilities. Psychotherapy Theory Research Practice Training 2003; 40:55-67.

28. Krijn M, Emmelkamp P, Olafsson RP, Biedmond R. Virtual reality exposure therapy of anxiety disorders: a review. Clinical Psychological Review 2004; 24:259-81.

29. Pull CB. Current status of virtual reality exposure therapy in anxiety disorders. Current Opinion in Psychiatry 2005; 18:7-14.

30. Gorini A, Riva G. Virtual reality in anxiety disorders: the past and the future. Expert Review of Neurotherapeutics 2008; 8(2):215-33. 
General introduction

31. Wiederhold B, Wiederhold MD. (2004) Virtual Reality Therapy for Anxiety Disorders: Advances in Evaluation and Treatment. American Psychological Association (APA). 



\section{CHAPTER II}

\section{The role of immersion and narrative in mediated presence: the virtual hospital experience}

A. Gorini, C. S. Capideville, G. De Leo, F. Mantovani, G. Riva.

CyberPsychology, Behaviour and Social Network. 2010, Jul 22. 



\section{Abstract}

The "mediated sense of presence" is a technology-induced illusion of being present in one (simulated) place when one is actually present in another (physical) place. Typically experienced in virtual worlds, mediated presence is generated by different technological, cognitive and emotional factors. The aim of this study was to test how to optimise the virtual experience manipulating some of these factors. Specifically, we tested if an immersive technology and/or a meaningful narrative context influence the users' sense of presence providing a more compelling experience than a non-immersive and non-contextualized virtual space.

Eighty-four students, randomly divided in four groups, were asked to find a blood container inside a virtual hospital in an immersive or non-immersive condition and with or without an emotionally related narrative. Two presence questionnaires and heart rate variations were used to measure the effects of the four conditions on the users' sense of presence.

Results showed a significant effect of narrative $(F(8,73)=30.346$, $\mathrm{p}<0.001)$, and level of immersion $(\mathrm{F}(8,73)=10.913, \mathrm{p}<0.001)$, and a significant interaction between narrative and immersion $(\mathrm{F}$ $(8,73)=2.062, p=0.051)$ both on the presence questionnaires and on the heart rate variations. Moreover, considering the factors immersion (I) and narrative (N) together we found that the I-N condition generates the highest level of presence.

We argue that both immersion and narrative are important to create an effective virtual reality experience, because they differently contribute to increase the sense of presence. Immersion increases the place illusion, while the narrative contributes to generate an emotional response and to strengthen the subjects' sense of inner presence. 


\section{Introduction}

Virtual Reality (VR) is a computer-simulated environment where users can explore and interact with an artificial reality having the feeling of being in a place different from where they physically are. A VR system is made of hardware, software, a user interface, and human factors, such as perceptions, cognition, and emotions.

From a technological point of view, VR can be immersive, when the user interact with it using a Head Mounted Display (HMD) and a tracker position sensor, or non-immersive, when the HMD is substituted by an external monitor. The HMD is a display device that presents the computer-generated images to each eye separately giving the illusion of a three-dimensional space and depth of field. Worn on the head or as part of a helmet, it covers the users' eyes, isolating them from the real world. When coupled with a tracker position sensor, the HMD becomes also an input device that transmits the user's movements to the computer that continuously update the virtual environments according to the user's point-of-view. Being the key element that distinguishes VR from the other existing media ${ }^{1}$, immersion provided by the HMD is supposed to play an important role in creating a successful virtual experience ${ }^{2}$.

As stated by Dourish ${ }^{3}$ VR is a technology that allows physical and virtual spaces to exist in parallel. But VR cannot be considered just as a pure technology ${ }^{4}$. VR is also an experiential interface whose contents are threedimensional environments (virtual worlds) where subjects interact between themselves and with the environment as if they were really inside it ${ }^{5}$. From a psychological point of view, the impression of "being there" has been defined "sense of presence" 6,7 . When experienced in a virtual reality setting, it is more precisely termed "mediated presence", indicating a technology-induced illusion of being present in one (simulated) place when one is actually present in another (physical) place ${ }^{8}$.

Even if many researches have focused their attention on the concept of mediated presence, we still lack a clear definition of the factors that contribute to generate it. In particular, there are two main theoretical approaches to the definition of presence. The first one defines it as a "perceptual illusion of non-mediation" 9 produced by means of the disappearance of the medium from the conscious attention of the subject. In this vision, the "Place Illusion", that is the sensation of being in a real place, is considered the main component that contributes to the realistic response during a virtual experience. Place illusion is constrained by the sensorimotor 
contingencies afforded by the virtual reality system and is strictly related to its level of immersion ${ }^{2}$.

The alternative approach in the definition of presence considers it as "Inner Presence", a broad psychological phenomenon, not necessarily linked to the experience of a medium, whose goal is the control of the individual and social activity ${ }^{7,10-23}$. This approach considers presence as the result of an evolved neuropsychological process ${ }^{8}$, influenced by cognitive and emotional factors, that play a crucial role in the way we differentiate between the internal and the external, make our subjective judgments, react to the surrounding world, attribute a meaning to our experience, and learn things about it. Technical details, such as system resolution, immersion, speed and kind of interface, as well as the ability of the designer contribute to the quality of the virtual representation, but the real author of the virtual experience is the user, who generates it driving its narrative flow through his/her own cognitive and emotional interpretation of the events ${ }^{1}$. When the user is emotionally and intellectually engaged in the experience and perceives a significant sense of control over the computer, his/her attention will focus on the interaction with the environment that generates an illusion of non-mediation increasing his/her sense of presence ${ }^{24}$. A key element of this process that combines form and content, is given by the introduction of narratives in the virtual contexts. Narratives are stories that users can inhabit from a first person perspective. They create meaning for the individual's experiences influencing the way people will appraise them, and change the individual's emotional states, modifying the way people evaluate the experience. According to Sherman and Craig ${ }^{1}$, narratives are responsible for mental immersion, through which users can be deeply engaged and involved in the experience, increasing their sense of mediated presence. In fact, when users identify themselves with the characters of the story, their suspension of disbelief makes the content of the virtual experience seem real. Because emotions have a very peculiar way of controlling minds and influencing people's actions, motivating learning and exploration behaviours, narratives that attribute an emotional content to the virtual experience are supposed to contribute to a higher sense of presence and a better interaction with the environment than neutral ones ${ }^{25,26}$.

Starting from these considerations, the aim of this study was to experimentally test how to optimise the virtual experience manipulating its technological (level of immersion), and psychological factors (narratives). In particular, we tested if an immersive technology and/or a meaningful narrative context influence the users' sense of presence providing a more compelling experience than a non-immersive and non-contextualized virtual 
space. In order to test our hypotheses, subjects were presented a virtual hospital and asked to find some blood containers in an immersive or nonimmersive modality associated or not to an emotionally narrative context. In particular, we investigated if:

1. The immersive condition provides a higher sense of presence than the non-immersive condition;

2. The immersive condition provides a higher emotional response (in terms of physiological activation) than the non-immersive condition;

3. The narrative context increases the sense of presence, in contrast with the non-narrative condition;

4. The narrative context increases the emotional response (in terms of physiological activation) experienced in the environment, in contrast with the non-narrative condition.

\section{Materials and Methods}

\section{Subjects}

Eighty-four undergraduate students (42 males and 42 females) between 19 and 25 (mean $=21,45$ years; $\mathrm{SD}=2,91$ ) years old participated in the study. All of them were familiar with technology and were used to using computer at least 5 days a week. They were randomly assigned to one of the following experimental groups (see tab. 1):

- Group 1: A virtual hospital was presented to the subjects in an immersive condition associated to an emotionally related narrative (I-N group);

- Group 2: A virtual hospital was presented to the subjects in an immersive condition without the narrative (I-NN group);

- Group 3: A virtual hospital was presented to the subjects in a nonimmersive condition associated to an emotionally related narrative (NI-N group);

- Group 4: A virtual hospital was presented to the subjects in a nonimmersive condition and without the narrative (NI-NN group).

Students with a history of neurological disease, head injury, learning disability, psychological disorders, and those who used psychotropic medications were excluded from the study. All subjects were volunteers and did not receive any payment or credit for their participations. An informed consent was obtained before starting the experiment. 
Immersion and narrative in mediated presence

\begin{tabular}{|l|l|l|}
\hline & Narrative (N) & Non-Narrative (NN) \\
\hline Immersive (I) & 21 participants (IN) & 21 participants (I-NN) \\
\hline Non-Immersive (NI) & 21 participants (NI-N) & 21 participants (NI-NN) \\
\hline
\end{tabular}

Tab. 1. Participants' distribution among the four experimental conditions.

\section{The virtual hospital}

The virtual hospital, developed using the 3D Game Studio 6 software, run on a portable computer (Sony Vaio Notebook PCG-GRT 996ZP, Pentium 4 $3,20 \mathrm{GHz}$ ) equipped with a graphic card NVIDIA GeForce FX Go5600 with $3 \mathrm{D}$ performance and $64 \mathrm{MB}$ of VRAM. The virtual environment represents a two-floor hospital connected by a lift (fig. 1). Many corridors and several rooms were located on each of the two floors. A big park and an ambulance parking surrounded the hospital. All subjects explored the same environment, but those included in the narrative conditions performed the task in the role of a doctor who had to find and bring a container with a rare type of blood back to the main hospital where a child was waiting for a lifesaving transfusion. Moreover, subjects in the narrative conditions were told that a mad murderer was wandering around the hospital trying to kill them so that they could not reach the blood containers needed to save the child (see the Experimental procedure section). Differently, subjects in the nonnarrative conditions were just asked to find the blood-containers exploring the virtual hospital, without any other specification that contextualized their task. In order to make the environment in the two conditions (narrative and non-narrative) as similar as possible they also met the virtual character representing the mad murderer, but without knowing anything about him.

The environment was presented on an external computer screen (Onyxblack LCD 16,1" UXGA) with a resolution of $1600 \times 1200$ in the nonimmersive condition, and through a Head Mounted Display (V-Real Viewer PC) with a resolution of $640 \times 480$ in the immersive condition. Participants navigated the environment using the joystick (non-immersive condition) or the joystick and the motion tracker integrated in the HMD (immersive condition). 


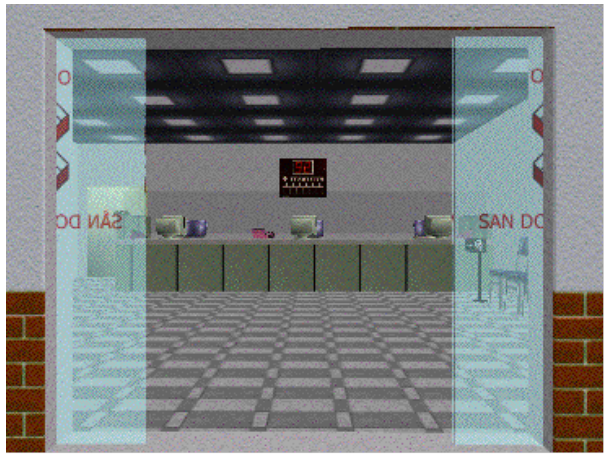

(a)

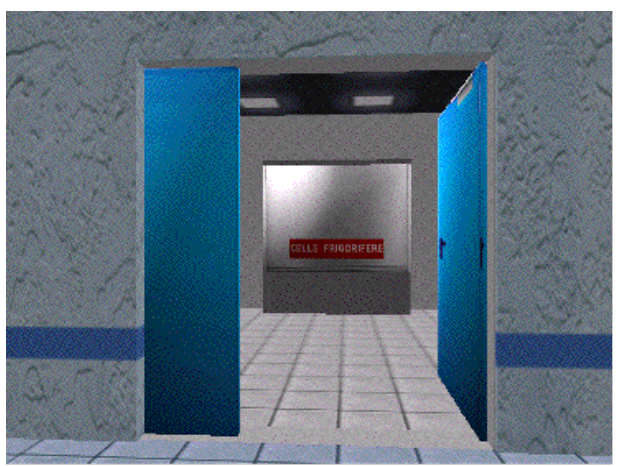

(c)

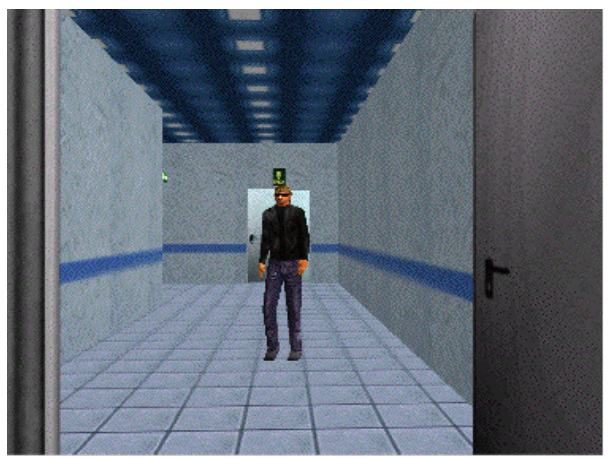

(b)

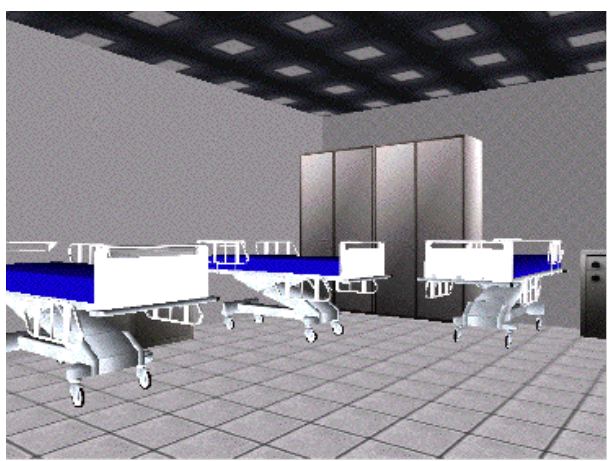

(d)

Fig. 1. The virtual hospital. (a) The hospital entrance; (b) The mad murderer wandering about the hospital; (c) The room with the blood container refrigerator; (d) One of the hospital room.

\section{Experimental design}

The independent variables of the study were: Narrative (exploration with a narrative context vs exploration without a narrative context) and Immersion (HMD - high degree of immersion vs laptop screen - low degree of immersion). The measured dependent variables were:

- the sense of presence (in terms of level of immersion): measured with the ITC-SOPI and the UCL self reported questionnaires;

- the physiological emotional response: assessed by the heart rate (HR) recording (see below for details).

\section{Presence evaluation}

The dependent variables were assessed using the following questionnaires:

- UCL Presence Questionnaire ${ }^{27}$ : a post-experience subjective measure of presence. Participants were required to provide 
ratings on a 1-7 Likert scale to the following questions: Q1"...rate your sense of being in the virtual environment; Q2- "To what extent were there times during the experience when the virtual environment was the reality for you?"; and Q3- "When you think back to the experience, do you think about the virtual environment more as images that you saw or as places that you visited?"

- Independent Television Company Sense of Presence Inventory (ITC-SOPI) ${ }^{28}$ : this post-exposure presence measure is divided in two parts. Part A is composed of 6 items and refers to the participants' impressions/feelings that follow the virtual experience. Part B consists of 38 items and refers to the subjects' impressions/feelings during the virtual experience. A consistent scoring mechanism (1-5 point Likert scale, from strongly disagree to strongly agree) is used for both parts. Factor analysis of this 44-item questionnaire showed that it measures the following dimensions of presence: a) Spatial presence - how physically present users feel in the virtual environment, b) Engagement how involved users would feel toward the content of the virtual environment, c) Ecological validity - the level of realism and naturalness of the environment.

As stated by Slater ${ }^{2}$ mediated presence is a qualia and there is no way to directly measure it. However, indirect assessments based on questionnaires that compare responses with those that would have been expected in real experiences can be considered the most acceptable indirect measurement of it.

\section{Psychophysiological evaluation}

The Procomp Infiniti Biofeedback system was used to record the heart rate at the baseline and during the exploration of the virtual hospital.

\section{Experimental procedure}

Participants were asked to seat in a swivel armchair in front of a laptop screen. At the beginning of the session they received one of the two sets of instructions according to the assigned group. Subjects in the non-narrative (NN) condition were simply told their goal was to find a blood container kept in the hospital refrigerator heedless of the other characters eventually present in the environment. Subjects in the narrative $(N)$ condition had the same goal, with the difference that it was contextualized in the following 
narrative: "A child has been involved in a car accident in front of the hospital, and if she does not receive an urgent blood transfusion, she would die. You are a doctor and you have to find the blood containers as soon as possible in order to save the child's life. Be careful, because a very dangerous mad murderer is wandering around the hospital trying to kill you before you reach the blood containers needed to save the child. When you find him, run away to prevent him to stop you before you find the blood". Due to its emotional significance, the encounter with the mad murderer was considered a significant moment of the virtual exploration, and marked during the HR registration (HR-ENC).

The experiment was divided into 2 parts:

Baseline: In this phase participants were requested to stay completely relaxed while the HR was recorded for 2 minutes ${ }^{29,30}$. Immediately after that, participants read the experiment instructions and were instructed about the use of the HMD equipment (if included in the I-N or I-NN groups) and on how to explore the virtual environment.

Navigation: In this phase, participants explored the virtual hospital until they found the blood containers, while their heart rate variations were recorded. At the end of the task, participants completed the UCL and the ITC-SOPI questionnaires in order to assess the sense of presence elicited by the navigation in the virtual environment. Finally, a debriefing phase concluded the session.

\section{Results}

We performed a multivariate analysis of variance (MANOVA) with immersion and narrative as independent factors, and the 3 UCL items, the 3 ITC-SOPI items, the HR variation (HR mean during the exploration of the environment minus HR mean at baseline), and the HR values recorded when the subjects encountered the mad murderer (HR-ENC) as dependent variables. Results showed a significant effect of narrative $(F(8,73)=$ $30.346, p<0.001)$, and level of immersion $(\mathrm{F}(8,73)=10.913, \mathrm{p}<0.001)$, and a significant interaction between narrative and immersion $(\mathrm{F}(8,73)=$ 2.062, $\mathrm{p}=0.051)$. Analyses of each individual dependent variable are reported in tab. 2. They show that the two Narrative groups $(\mathrm{N})$ and the two Immersion (I) groups significantly differed in almost all the analyzed dependent variables except in the ITC-SOPI item "Ecological validity" (in the $\mathrm{N}$ groups) and in the HR when subjects encountered the mad murderer 
(in the I groups). The ITC-SOPI and the HR values were also significant in the interaction analysis (Narrative * Immersion).

Looking at the mean values calculated in the four experimental groups and reported in tab. 3 we notice that:

- In all the presence questionnaires, the means recorded in the I-N condition are the highest values, while the means in the NI-NN condition are the lowest, except in the ITC-SOPI Ecological validity item in which the I-N condition is not significantly different from the I-NN group;

- Regarding the HR values, the I-N condition is not significantly different from the NI-N condition, but it is higher than the I-NN and NI-NN both in HR and HR-ENC.

\begin{tabular}{|l|l|r|r|r|}
\hline Condition & \multicolumn{1}{|c|}{ Dependent Variable } & \multicolumn{1}{c|}{ df } & \multicolumn{1}{c|}{ F } & \multicolumn{1}{c|}{ p } \\
\hline Narrative & UCL Q1 & 1,80 & 14,222 &, $000^{*}$ \\
\cline { 2 - 5 } & UCL Q2 & 1,80 & 12,738 &, $001^{*}$ \\
\cline { 2 - 5 } & UCL Q3 & 1,80 & 21,878 &, $000^{*}$ \\
\cline { 2 - 5 } & ITC-SOPI Spatial presence & 1,80 & 15,563 &, $000^{*}$ \\
\cline { 2 - 5 } & ITC-SOPI Ecological validity & 1,80 & 3,212 &, 077 \\
\cline { 2 - 5 } & ITC-SOPI Engagement & 1,80 & 17,464 &, $000^{*}$ \\
\cline { 2 - 5 } & HR (exploration - baseline) & 1,80 & 20,341 &, $000^{*}$ \\
\cline { 2 - 5 } & HR-ENC & 1,80 & 173,769 &, $000^{*}$ \\
\hline \multirow{5}{*}{ Immersion } & UCL Q1 & 1,80 & 14,349 &, $000^{*}$ \\
\cline { 2 - 5 } & UCL Q2 & 1,80 & 16,001 &, $000^{*}$ \\
\cline { 2 - 5 } & UCL Q3 & 1,80 & 9,885 &, $002^{*}$ \\
\cline { 2 - 5 } & ITC-SOPI Spatial presence & 1,80 & 19,501 &, $000^{*}$ \\
\cline { 2 - 5 } & ITC-SOPI Engagement & 1,80 & 20,075 &, $000^{*}$ \\
\cline { 2 - 5 } & ITC-SOPI Ecological validity & 1,80 & 17,915 &, $000^{*}$ \\
\cline { 2 - 5 } & HR (exploration - baseline) & 1,80 & 4,187 &, $044^{*}$ \\
\cline { 2 - 5 } & HR-ENC & 1,80 &, 040 &, 841 \\
\hline Narrative * & UCL Q1 & 1,80 &, 967 &, 328 \\
\cline { 2 - 5 } & UCL Q2 & 1,80 &, 347 &, 557 \\
\cline { 2 - 5 } & UCL Q3 & 1,80 & 2,612 &, 110 \\
\cline { 2 - 5 } & ITC-SOPI Spatial presence & 1,80 & 1,199 &, 277 \\
\cline { 2 - 5 } & ITC-SOPI Engagement & 1,80 &, 688 &, 409 \\
\cline { 2 - 5 } & ITC-SOPI Ecological validity & 1,80 & 5,327 &, $024 *$ \\
\cline { 2 - 5 } & HR (exploration - baseline) & 1,80 &, 007 &, 933 \\
\cline { 2 - 5 } & HR-ENC & 1,80 & 6,491 &, $013^{*}$ \\
\hline
\end{tabular}

Tab. 2. Df, F, and p values obtained from the MANOVA using Narrative and Immersion as independent variables. 
Chapter II

\begin{tabular}{|c|c|c|c|c|c|}
\hline Dependent Variable & $\begin{array}{c}\text { Group } \\
\text { (I) }\end{array}$ & $\begin{array}{c}\text { Group } \\
\text { (J) }\end{array}$ & Mean & SD & Sig. \\
\hline \multicolumn{6}{|l|}{ Presence evaluation } \\
\hline \multirow[t]{4}{*}{ UCL Q1 } & \multirow[t]{4}{*}{$\mathrm{I}-\mathrm{N}$} & $\mathrm{I}-\mathrm{N}$ & 5,48 & 1,17 & - \\
\hline & & $\mathrm{I}-\mathrm{NN}$ & 4,76 & 1,51 &, $043^{*}$ \\
\hline & & NI-N & 4,48 & 1,33 &, $045^{*}$ \\
\hline & & NI-NN & 3,38 & 1,28 &, $000^{*}$ \\
\hline \multirow[t]{4}{*}{ UCL Q2 } & \multirow[t]{4}{*}{ I-N } & $\mathrm{I}-\mathrm{N}$ & 5,57 & 1,12 & - \\
\hline & & $\mathrm{I}-\mathrm{NN}$ & 4,14 & 1,65 &, $022^{*}$ \\
\hline & & NI-N & 3,57 & 1,50 &, $009^{*}$ \\
\hline & & NI-NN & 2,95 & 1,32 &, $000^{*}$ \\
\hline \multirow[t]{4}{*}{ UCL Q3 } & \multirow[t]{4}{*}{ I-N } & $\mathrm{I}-\mathrm{N}$ & 5,81 & 1,44 & - \\
\hline & & I-NN & 4,19 & 1,46 &, $000^{*}$ \\
\hline & & $\mathrm{NI}-\mathrm{N}$ & 3,86 & 1,99 &, $006^{*}$ \\
\hline & & NI-NN & 2,67 & 1,24 &, $000^{*}$ \\
\hline \multirow[t]{4}{*}{ ITC-SOPI Spatial presence } & \multirow[t]{4}{*}{ I-N } & $\mathrm{I}-\mathrm{N}$ & 64,09 & 5,18 & - \\
\hline & & I-NN & 55,57 & 9,32 & ,003* \\
\hline & & NI-N & 52,52 & 11,60 &, $001^{*}$ \\
\hline & & NI-NN & 46,42 & 8,55 &, $000^{*}$ \\
\hline \multirow[t]{4}{*}{ ITC-SOPI Ecological validity } & \multirow[t]{4}{*}{ I-N } & $\mathrm{I}-\mathrm{N}$ & 52,04 & 3,40 & - \\
\hline & & $\mathrm{I}-\mathrm{NN}$ & 47,95 & 6,93 & 256 \\
\hline & & NI-N & 47,23 & 7,00 &, $002 *$ \\
\hline & & NI-NN & 43,33 & 8,83 &, $000^{*}$ \\
\hline \multirow[t]{4}{*}{ ITC-SOPI Engagement } & \multirow[t]{4}{*}{$\mathrm{I}-\mathrm{N}$} & $\mathrm{I}-\mathrm{N}$ & 22,47 & 3,65 & - \\
\hline & & I-NN & 18,71 & 3,71 &, $000^{*}$ \\
\hline & & NI-N & 17,76 & 4,78 &, $000^{*}$ \\
\hline & & NI-NN & 15,42 & 4,69 &, $000^{*}$ \\
\hline \multicolumn{6}{|l|}{ Psychophysiological evaluation } \\
\hline \multirow[t]{4}{*}{ HR (exploration - baseline) } & \multirow[t]{4}{*}{$\mathrm{I}-\mathrm{N}$} & $\mathrm{I}-\mathrm{N}$ & 13,18 & 10,66 & - \\
\hline & & I-NN & 2,95 & 6,23 &, $009^{*}$ \\
\hline & & NI-N & 9,19 & 8,62 & 438 \\
\hline & & NI-NN & 2,08 & 2,66 &, $000^{*}$ \\
\hline \multirow[t]{4}{*}{ HR-ENC } & \multirow[t]{4}{*}{ I-N } & I-N & 114,64 & 10,78 & - \\
\hline & & I-NN & 83,57 & 8,48 & ,000* \\
\hline & & NI-N & 107,41 & 12,73 &, 352 \\
\hline & & NI-NN & 86,84 & 8,25 &, $000^{*}$ \\
\hline
\end{tabular}

Tab. 3. Mean values and Tukey HSD post hoc tests calculated for each dependent variable.

\section{Discussion}

The main goal of the present study was to investigate which elements enhance the users' sense of mediated presence during a virtual reality experience. To do it, we analysed the contribution of technological (i.e. the 
physical immersion through the use of the HMD) and cognitive factors (i.e. the narrative) during the exploration of a virtual hospital.

\section{The role of immersion}

Consistently with our hypotheses, the results show a significant influence of physical immersion on all the presence self reported questionnaires suggesting its role in increasing one of the aspect of presence, defined by Slater ${ }^{2}$ as place illusion. Compared to those who experienced the virtual environment in a non-immersive condition, participants who used the HMD showed higher mean scores in all the UCL and ITC-SOPI items. In the immersive condition, characters and objects, as well as the environment itself, were perceived as more real, and the experience was judged more interesting and involving than in the non-immersive condition. Wearing the HMD participants felt to be more present inside the environment and believed they could change the events interacting with characters and objects more than those in the non-immersive condition. Nevertheless, the level of immersion seems to be not sufficient to alter the subjects' physiological response, probably because it does not really contribute to the contextualization of the events, that is a key factor of any vivid emotional experience.

\section{The role of the narrative}

Providing a context to the virtual hospital navigation, the presence of the narrative had a significant effect both on the presence self reported questionnaires, and on the heart rate variations, increasing what has been defined by Waterworth, the users' sense of inner presence ${ }^{8}$. In particular, compared to those in the non-narrative groups, and regardless the level of immersion, participants in the narrative conditions showed a higher HR variation between the baseline and the virtual exploration, and a higher heart rate activation when they encountered the virtual character representing the mad murderer. These results are consistent with the Healey's theory on emotions ${ }^{31}$ affirming that an increasing in the heart rate response is an important physiological indicator of arousal and valence activation. In fact, giving a specific role to the participants - being a doctor that must save a little child finding the blood containers - and attributing negative characteristics to the character found inside the hospital - he was described as a dangerous mad murderer - significantly increased their emotional states. The narrative context leaded the two groups of participants $(\mathrm{N}$ vs $\mathrm{NN}$ ) to a different evaluation of the experience and modified their appraisal accordingly ${ }^{32}$. In fact, even if all subjects were asked to perform exactly the 
same task (to find the blood containers), only those in the narrative conditions had a motivation to find them that gave more relevance to the experience. Similarly, even if all participants found a man inside the hospital, only those in the narrative condition knew that he was a murderer that could impede them to reach their goal. This information caused a significant alteration in their emotional response, according to the Fridja's theory ${ }^{33}$ affirming that the emotion elicitation depends on the way people appraise situations.

The only measured variable on which we did not find a significant effect of the narrative was the ICT-SOPI Ecological validity item that measures the level of realism and naturalness of the environment. This result can be reasonably explained by the fact that the realism and naturalness of a virtual environment are physical characteristics more related to the way in which we perceive the environment than to the way in which we contextualize it. Considering the factors immersion and narrative together and combining the four conditions (immersion, non-immersion, narrative, non-narrative) we found that the I-N condition is the one that generates the maximum level of mediated presence, both in terms of place illusion and inner presence. On the contrary, the condition that produces the minimum level of presence is the NI-NN. Given these results, we argue that both immersion and narrative are important features that must be taken into account in order to create an effective virtual reality experience, because they differently contribute to the theoretical construct defined "sense of presence". Immersion increases the place illusion, that is the strong illusion of being in a place in spite of the sure knowledge to be not there, while a narrative that contextualizes the virtual experience generates an action based and controlled by the subject's needs, motives and goals that significantly contributes to generate an emotional response and to strengthen the sense of inner presence. Both the place illusion and the inner presence participate in the generation of the mediated presence, that requires adequate form to be directly perceived, conscious attention to that form, and content that will sustain such attention. Previous studies had already investigated the impact of specific elements, such as the width of the field of view ${ }^{34}$ or the emotional content provided by the virtual environment ${ }^{35}$ on the users' sense of presence, but this is the first attempt to simultaneously addresses the combination of technical and psychological aspects. Nevertheless, a possible fragility of this study that should be addressed in future researches is that we did not evaluate the presence, in our sample, of Blood-Injection-Injuries phobia, a subtype of specific phobias that includes, among the others, the fear of blood (hemophobia). Since participants were asked to look for blood containers, 
the possible presence of this kind of specific fear could be a confounding variable, because high levels of fear are known to also increase the sense of presence during VR stimulation ${ }^{36,37}$. Anyway, since this pathological condition is rare, we suppose that our results have not been altered by it.

In conclusion, to obtain a high level of mediated presence, the virtual environment should be presented in immersive condition and associated with a contextualized narrative, creating equilibrium between technological and cognitive elements. Nevertheless, our data suggest that, while immersion itself produces an increased presence as measured with the self reported questionnaires, but does not alter the physiological response, the narrative affects both the responses to the questionnaires and the subjects' physiological activation. Other than increasing the proto (proprioceptive) presence and the core (perceptual) presence, the emotional content transmitted by the narrative also increases the extended (reflective) presence producing a significant sense of inner presence ${ }^{8}$. 


\section{References}

1. Sherman WR, Craig AB. (2003) Understanding virtual reality: Interface, application, and design. San Francisco, CA: Morgan Kaufmann Publishers.

2. Slater M. Place illusion and plausibility can lead to realistic behaviour in immersive virtual environments. Philos Trans R Soc Lond B Biol Sci 2009; Dec 12; 364(1535):3549-57.

3. Dourish P. (2001) Where the action is: The foundations of embodied interaction. Cambridge, MA: MIT Press.

4. Steuer J. Defining virtual reality: dimensions determining telepresence. Journal of Communication 1992; 42(4):73-93.

5. Riva G, Waterworth JA, Waterworth EL. The layers of presence: a bio-cultural approach to understanding presence in natural and mediated environments. Cyberpsychology \& Behaviour 2004; 7(2):405-19.

6. Coelho CM, Tichon JG, Hine TJ, Wallis G, Riva G. (2006) Media presence and inner presence: The sense of presence in virtual reality technologies. In: Riva G, Anguera MT, Wiederhold BK, Mantovani F (eds). From Communication to Presence Amsterdam: IOS Press, 25-45.

7. Riva G, Davide F, Ijsslsteijn WA (eds). Being there: Concepts, effects and measurements of user presence in synthetic environments. Amsterdam: IOS Press; 2003.

8. Waterworth JA, Waterworth EL, Mantovani F, Riva G. On feeling (the) present. Journal of Consciousness Studies 2010; 17(1-2).

9. Lombard M, Ditton T. At the heart of it all: the concept of presence. Journal of Computer Mediated-Communication 1997; 3(2).

10. Banos RM, Botella C, Garcia-Palacios A, Villa H, Perpina C, Alcaniz M. Presence and reality judgment in virtual environments: a unitary construct? Cyberpsychology \& Behaviour 2000; 3(3):32755 .

11. Banos RM, Botella C, Perpina C. Virtual reality and psychopathology. Cyberpsychol Behav 1999; 2(4):283-92.

12. Lee KM. Presence, explicated. Journal of Communication 2004; 14:27-50.

13. Lee KM. Why presence occurs: evolutionary psychology, media equation, and presence. Presence 2004; 13(4):494-505. 
14. Mantovani F, Riva G. "Real" presence: how different ontologies generate different criteria for presence, telepresence, and virtual presence. Presence, Teleoperators, and Virtual Environments 1999; 8(5):538-48.

15. Marsh T, Wright P, Smith S. Evaluation for the design of experience in virtual environments: modeling breakdown of interaction and illusion. Cyberpsychology \& Behaviour 2001; 4(2):225-38.

16. Moore K, Wiederhold BK, Wiederhold M, Riva G. Panic and agoraphobia in a virtual world. Cyberpsychology \& Behaviour 2002; 5(3):197-202.

17. Riva G, Davide F (eds). Communications through virtual technologies: Identity, community and technology in the communication age. Amsterdam: IOS Press; 2001.

18. Riva G, Waterworth JA, Waterworth EL, Mantovani F. From intention to action: The role of presence. New ideas in psychology In press.

19. Schubert $T$, Friedman F, Regenbrecht H. The experience of presence: factor analytic insights. Presence: Teleoperators, and Virtual Environments 2001; 10(3):266-81.

20. Spagnolli A, Gamberini L, Gasparini D. Breakdown analysis in virtual reality usability evaluation. PsychNology Journal 2003; 1(1).

21. Waterworth EL, Ha"ggkvist M, Jalkanen K, Olsson S, Waterworth JA, Wimelius $\mathrm{H}$. The exploratorium: an environment to explore your feelings. PsychNology Journal 2003; 1(3):189-201.

22. Waterworth JA, Waterworth EL. Focus, locus, and sensus: the three dimensions of virtual experience. Cyberpsychology \& Behaviour 2001; 4(2):203-13.

23. Zahoric P, Jenison RL. Presence as being-in-the-world. Presence, Teleoperators, and Virtual Environments 1998; 7(1):78-89.

24. Waterworth JA, Waterworth EL, Westling J. Presence as performance: The mystique of digital participation. Presence 2002: Fifth Annual International Workshop. Porto, Portugal, 2002.

25. Zajonc RB. Feeling and thinking: Preferences need no interferences. American Psychologist 1980; 35:151-75.

26. Isen AM. (2000) Positive Affect and Decision Making. In: Lewis M, Haviland-Jones JM (eds). Handbook of Emotions. New York: Guilford Press.

27. Slater M, Usoh M, Steed A. Depth of presence in virtual environments. Presence: Teleoperators and Virtual Environments 1994; 1:130-44. 
28. Lessiter J, Freeman J, Keogh E. Acrossmedia presence questionnaire: the ITC Sense of Presence Inventory. Presence: Teleoperators, and Virtual Environments 2001; 10:282-97.

29. Linden W. What do arithmetic stress tests measure? Protocol variations and cardiovascular responses. Psychophysiology 1991; 28:91-102.

30. Matthews KA, Stoney CM. Influences of sex and age on cardiovascular responses during stress. Psychosom Med 1988; 50:46-56.

31. Healey JA. (2000) Wearable and automative system for affect recognition from physiology. Boston, MA: MIT Press.

32. Scherer KR, Shorr A, Johnstone T (eds). Appraisal processes in emotion: theory, methods, research. Canary, NC: Oxford University Press; 2001.

33. Frijda NH. (1987) The emotions. Cambridge University Press.

34. Prothero J, Hoffman H. Widening the field of view increases the sense of presence. Human Interface Technology Lab, 1995.

35. Riva G, Mantovani F, Capideville CS, et al. Affective Interactions Using Virtual Reality: The Link between Presence and Emotions. Cyberpsychol Behav 2007; 10(1):45-56.

36. Regenbrecht HT, Schubert TW, Friedman F. Measuring the sense of presence and its relations to fear if heights in virtual environments. International Journal of Human-Computer Interaction 1998; 10:23349.

37. Hoffman HG, Prothero J, Wells MJ, Groen J. Virtual chess: meaning enhances user's sense of presence in virtual environments. International Journal of Human-Computer Interaction 1998; 10:25163. 


\section{CHAPTER III}

\section{Assessment of the emotional}

responses produced by exposure to real food, virtual food and photographs of food in patients affected by eating disorders

A. Gorini, E. Griez, A. Petrova, G. Riva.

Annals of General Psychiatry. 2010, Jul 5; 9(1):30. 



\section{Abstract}

\section{Background}

Many researchers and clinicians have proposed using virtual reality (VR) in adjunct to in vivo exposure therapy to provide an innovative form of exposure to patients suffering from different psychological disorders. The rationale behind the 'virtual approach' is that real and virtual exposures elicit a comparable emotional reaction in subjects, even if, to date, there are no experimental data that directly compare these two conditions. To test whether virtual stimuli are as effective as real stimuli, and more effective than photographs in the anxiety induction process, we tested the emotional reactions to real food (RF), virtual reality (VR) food and photographs $(\mathrm{PH})$ of food in two samples of patients affected, respectively, by anorexia (AN) and bulimia nervosa (BN) compared to a group of healthy subjects. The two main hypotheses were the following: (a) the virtual exposure elicits emotional responses comparable to those produced by the real exposure; (b) the sense of presence induced by the VR immersion makes the virtual experience more ecological, and consequently more effective than static pictures in producing emotional responses in humans.

\section{Methods}

In total, $10 \mathrm{AN}, 10 \mathrm{BN}$ and 10 healthy control subjects (CTR) were randomly exposed to three experimental conditions: $\mathrm{RF}, \mathrm{PH}$, and VR while their psychological (State Anxiety Inventory (STAI-S) and visual analogue scale for anxiety (VAS-A)) and physiological (heart rate, respiration rate, and skin conductance) responses were recorded.

\section{Results}

$\mathrm{RF}$ and VR induced a comparable emotional reaction in patients higher than the one elicited by the PH condition. We also found a significant effect in the subjects' degree of presence experienced in the VR condition about their level of perceived anxiety (STAI-S and VAS-A): the higher the sense of presence, the stronger the level of anxiety. 


\section{Conclusions}

Even though preliminary, the present data show that VR is more effective than PH in eliciting emotional responses similar to those expected in real life situations. More generally, the present study suggests the potential of VR in a variety of experimental, training and clinical contexts, being its range of possibilities extremely wide and customizable. In particular, in a psychological perspective based on a cognitive behavioral approach, the use of VR enables the provision of specific contexts to help patients to cope with their diseases thanks to an easily controlled stimulation. 


\section{Background}

In the last few years there have been many attempts to treat mental disorders using virtual reality (VR), an innovative technique that allows patients to virtually experience critical situations (for example, exposure to a phobic stimulus) in a very safe environment while under the direct supervision of their therapists (for recent reviews see [1-3]). Following a cognitive behavioral-based approach, therapists can take advantage of interactivity and flexibility offered by virtual environments to measure and monitor a wide variety of patients' responses in real time, overcoming the limitations usually encountered during the in vivo exposure. Differently from what happens in real life settings, virtual environments can be tailored to the patients' needs and/or to therapeutic scopes in order to create specific and highly controller exposure settings. Moreover, compared to the most used therapeutic approaches, such as guided imagination or exposure to photographic materials, VR allows subjects to interact and manipulate 3D environments, mimicking interaction with objects in the real world. This experience increases the ecological validity of the simulated environment and enhances the 'sense of presence', defined as 'the user's sense of "being there" in the virtual environment' [4], or 'a perceptual illusion of nonmediation' [5]. In other words, the sense of presence is what happens when users 'forget' that their perceptions are mediated by technologies, feeling part of the virtual world 'as it was real' [6]. Through the increasing of the sense of presence, patients experience vivid real-life recreations that offer them contextual cues and facilitate generalization [7-9].

Today there is a growing recognition that VR may play an important role in clinical psychology, being a valid alternative to real-life exposure. However, the 'virtual approach' can be accepted only if real and virtual exposures elicit a comparable emotional response in subjects [10]. In order to verify whether virtual stimuli are as effective as real ones, and more powerful than static photographs, we assessed the emotional responses to real food (RF), virtual reality (VR) foods and photographs of food $(\mathrm{PH})$ in two samples of patients affected, respectively, by anorexia (AN) and bulimia nervosa (BN), and in a sample of healthy controls (CTR). The reason why we chose food exposure is that, in addition to other situations of equal or more importance, it is one of the most typical conditions that provokes an emotional response in patients affected by eating disorders (ED) [11-14].

Various studies have used virtual stimuli instead of real ones to assess and treat eating behaviors in ED patients $[15,16]$, but the first systematic 
attempts to evaluate the usefulness of virtual environments in provoking emotional reactions in such patients were carried out by Ferrer-Garcia et al. and Gutierrez-Maldonado et al. [17, 18]. They created six virtual environments representing situations that are emotionally significant to subjects with eating disorders, and measured the level of state anxiety and depression in participants after exposure to each of them concluding that, upon simulation of real-life stressful situations, these environments are effective in producing significant emotional reactions in their users. Using a similar approach, but comparing the virtual stimuli directly with the real ones, and with their correspondent pictures, we wanted to test the psychological and physiological reactions to food in a sample of ED patients (half anorexic and half bulimic) and healthy controls. The two main hypotheses of the study were the following: (1) that the virtual exposure elicits emotional responses comparable to those produced by the real exposure, and (2) the sense of presence induced by the VR immersion makes the virtual experience more ecological, and consequently more effective than static pictures in producing emotional responses in humans.

\section{Methods}

\section{Subjects}

The experimental sample included 20 female patients affected by eating disorders (10 $\mathrm{AN}$ and $10 \mathrm{BN}$ ) and a control group of 10 healthy females (CTR) matched for age with the experimental groups. The mean body mass index (BMI) was $17.05 \pm 1.09$ in the AN group, $24.40 \pm 4.05$ in the BN group, and $21.82 \pm 2.50$ in the CTR group (see Table 1 for details).

Patients were randomly recruited from the outpatient units of two public Italian hospitals in Milan, Italy, while CTR subjects were recruited through local advertisements among college students, administrative and workers' staff of the hospitals. Exclusion criteria for the AN and BN groups were the presence of lifetime psychiatric diseases other than eating disorders, major medical diseases, neurological syndromes, and brain injury or trauma. Consensus diagnoses, according to the Diagnostic and Statistical Manual of Mental Disorders, fourth edition (DSM-IV) criteria, were obtained by two clinicians who independently assessed all patients using a clinical interview and the Mini International Neuropsychiatric Interview Plus (MINI) [19], a diagnostic instrument designed to meet the need for a short but accurate structured psychiatric interview for DSM-IV and ICD-10 disorders. The 
severity of eating symptoms was then assessed with the Eating Disorders Inventory 2 (EDI-2) [20] (see Table 2 and the section on Psychological assessment for details). The MINI was also administered to the CTR group in order to exclude the presence of any psychiatric diseases, including actual or past eating disorders. Control subjects who were following a diet at the moment of the experiment were also excluded from the study.

Subjects who gave their written informed consent to participate were included in the study. When participants were under 18, informed consent was obtained from their parents.

\begin{tabular}{lcccc}
\hline GROUP & MINIMUM & MAXIMUM & MEAN & SD \\
\hline Control $(\mathbf{N}=\mathbf{1 0})$ & & & & \\
\hline Age & 19 & 34 & 26.20 & 5.14 \\
\hline BMI & 18.01 & 25.80 & 21.82 & 2.50 \\
\hline ED $(\mathbf{A N})(\mathbf{N}=\mathbf{1 0})$ & & & & \\
\hline Age & 16 & 31 & 22.30 & 5.62 \\
\hline BMI & 15 & 18.1 & 17.05 & 1.08 \\
\hline ED (BN) $(\mathbf{N}=\mathbf{1 0})$ & & & & \\
\hline Age & 17 & 32 & 23.90 & 5.26 \\
\hline BMI & 18.45 & 30.60 & 24.40 & 4.05 \\
\hline
\end{tabular}

Tab. 1. Age and BMI averages of CTR and ED groups.

\begin{tabular}{lcc}
\hline & $\begin{array}{c}\text { AN } \\
\text { Mean (SD) }\end{array}$ & $\begin{array}{c}\text { BN } \\
\text { Mean (SD) }\end{array}$ \\
\hline EDI-2 & & \\
\hline Drive for thinness & $9.13(4.11)$ & $12.15(6.03)$ \\
\hline Bulimia & $3.01(3.49)$ & $9.13(7.01)$ \\
\hline Body dissatisfaction & $13.05(7.14)$ & $18.41(6.22)$ \\
\hline Ineffectiveness & $6.57(5.09)$ & $10.34(5.67)$ \\
\hline Perfection & $5.66(2.34)$ & $3.1(3.45)$ \\
\hline Interpersonal distrust & $6.70(4.56)$ & $5.50(3.9)$ \\
\hline Interceptive awareness & $8.03(5.67)$ & $11.34(8.43)$ \\
\hline Maturity fears & $4.23(3.98)$ & $6.23(4.53)$ \\
\hline Asceticism & $3.56(3.45)$ & $5.89(3.89)$ \\
\hline Impulse regulation & $4.34(4.49)$ & $7.03(5.79)$ \\
\hline Social insecurity & $8.09(5.89)$ & $7.98(6.35)$ \\
\hline
\end{tabular}

Tab. 2. EDI-2 averages of AN and BN groups. 
Assessment

Psychological assessment

The following questionnaires were administered to the participants before the experiment.

- EDI-2

EDI-2 [20], a self-report questionnaire that provides clinical information regarding the psychological and behavioral dimensions usually associated with anorexia and bulimia nervosa.

- State Anxiety Inventory (STAI-S)

The STAI-S was initially conceived as a research instrument for the study of anxiety in adults. According to the author, state anxiety reflects a 'transitory emotional state or condition of the human organism that is characterized by subjective, consciously perceived feelings of tension and apprehension, and heightened autonomic nervous system activity'. State anxiety may fluctuate over time and can vary in intensity, in contrast with the trait anxiety that denotes 'relatively stable individual differences in anxiety proneness...' and refers to a general tendency to respond with anxiety to perceived threats in the environment [21]. Scores on the STAI-S have a direct interpretation: high scores mean more state anxiety and low scores mean less.

\section{- Visual analogue scale for anxiety (VAS-A)}

The VAS-A [22] is a $100 \mathrm{~mm}$ vertical line with end points anchored as no anxiety at the bottom of the scale and anxiety as bad as it could possibly be at the top; scores range from 0 to 10 . Among the numerous tools available for assessing anxiety, direct scaling procedures, such as the VAS, are popular because of their simplicity, versatility, relative insensitivity to bias effects, and the assumption that the procedures yield numerical values that are valid, reliable, and on a ratio scale [23-25].

\section{- ITC-Sense of Presence Inventory (ITC-SOPI)}

The ITC-SOPI [26] is a validated questionnaire focusing on users' experiences of virtual reality (and media, in general) that evaluates the degree to which the subject experienced the 'sense of being in the virtual environment', how far the virtual environment was the dominant reality, and how far it is recalled as a 'place'.

Psychophysiological assessment

The Biograph Infiniti (Thought Technology Ltd, New York, USA) 
biofeedback equipment was used to measure the heart rate (HR) and respiration rate (RESP), and the skin conductance (SCR) of subjects before (baseline) and during exposure to food.

Experimental procedures

All subjects were presented to the following three conditions, outlined below.

Condition 1: real food view (RF)

Six real high-calorie foods (three savory and three sweet) (Figure 1) were presented for $30 \mathrm{~s}$ each with a pause of other $30 \mathrm{~s}$ between each other on a table in front of the subject. During the pause, all foods were covered with six red plastic lids so that subjects could not see them.

Condition 2: photograph slide show (PH)

A slideshow presentation including the photographs of the same six foods presented in the RF condition was presented on a computer screen. The presentation time and the interval between the different pictures were the same used in the RF condition. During the $30 \mathrm{~s}$ pauses a picture of the red lid covering a hidden food appeared on the screen.

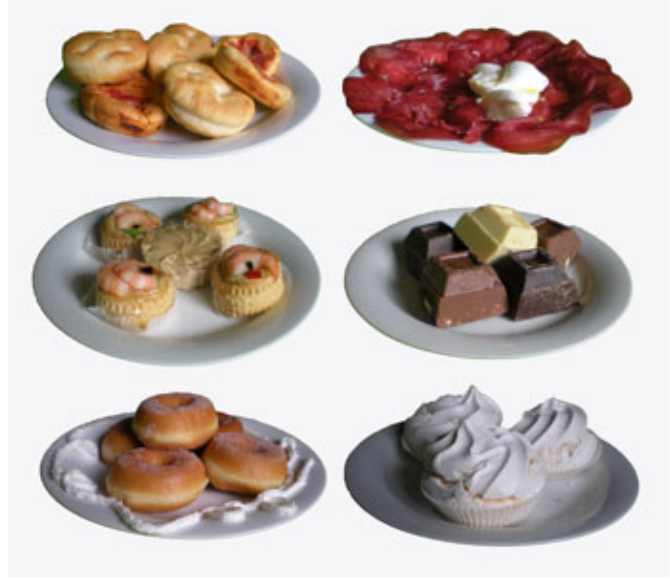

Fig. 1. The six high-calorie foods (three savory and three sweet foods) presented to the subjects in the three experimental conditions.

Condition 3: virtual reality (VR) immersive condition

In the VR condition subjects were asked to wear a head mounted display 
(HMD) in order to have a 3D view of the virtual environment. The motion tracker included in the HMD and a joystick allowed them to explore the environment and to interact with the virtual food. The environment represented a small restaurant with a buffet table in it (the virtual restaurant is included in NeuroVR [27], free open source software available at http://www.neurovr.org). A virtual representation of the same six foods presented in the RF and PH conditions appeared on the restaurant table and subjects were asked to explore the environment and to virtually open the lids one by one observing the food for $30 \mathrm{~s}$, as happened in the two other conditions (Figure 2).

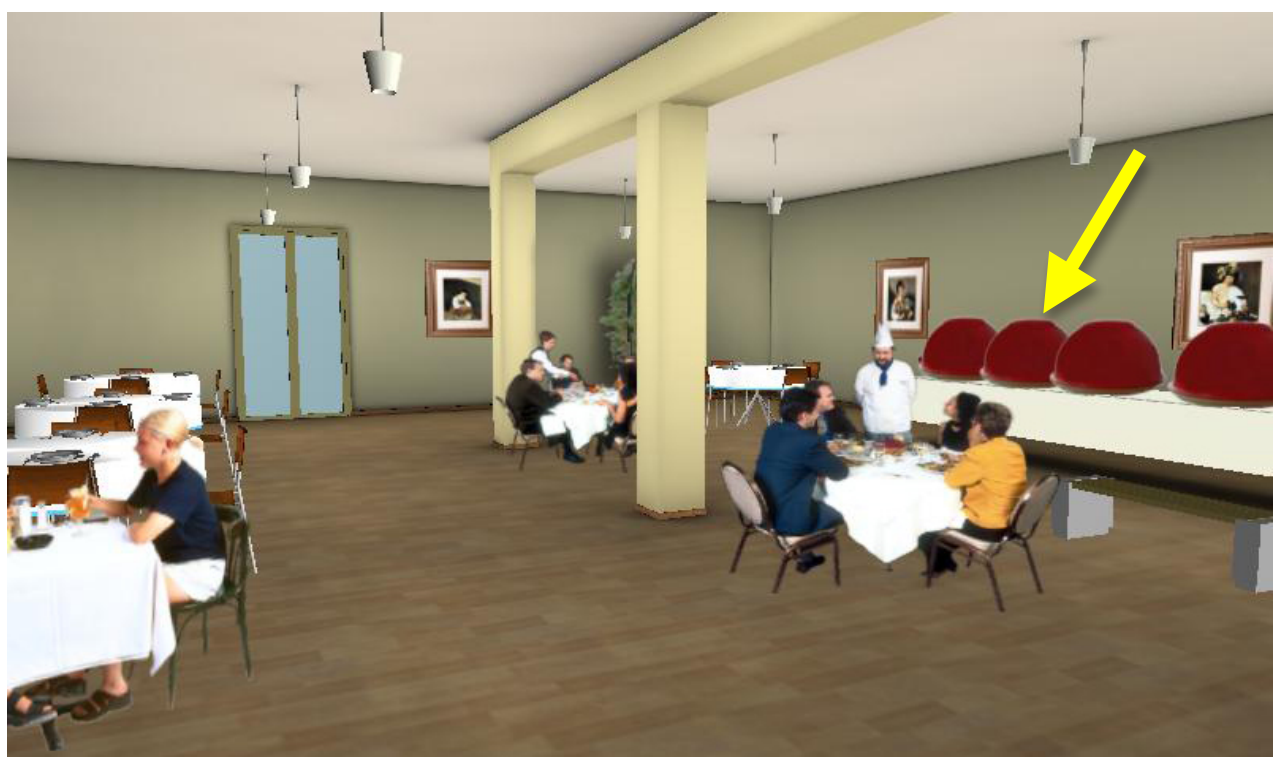

(a) 


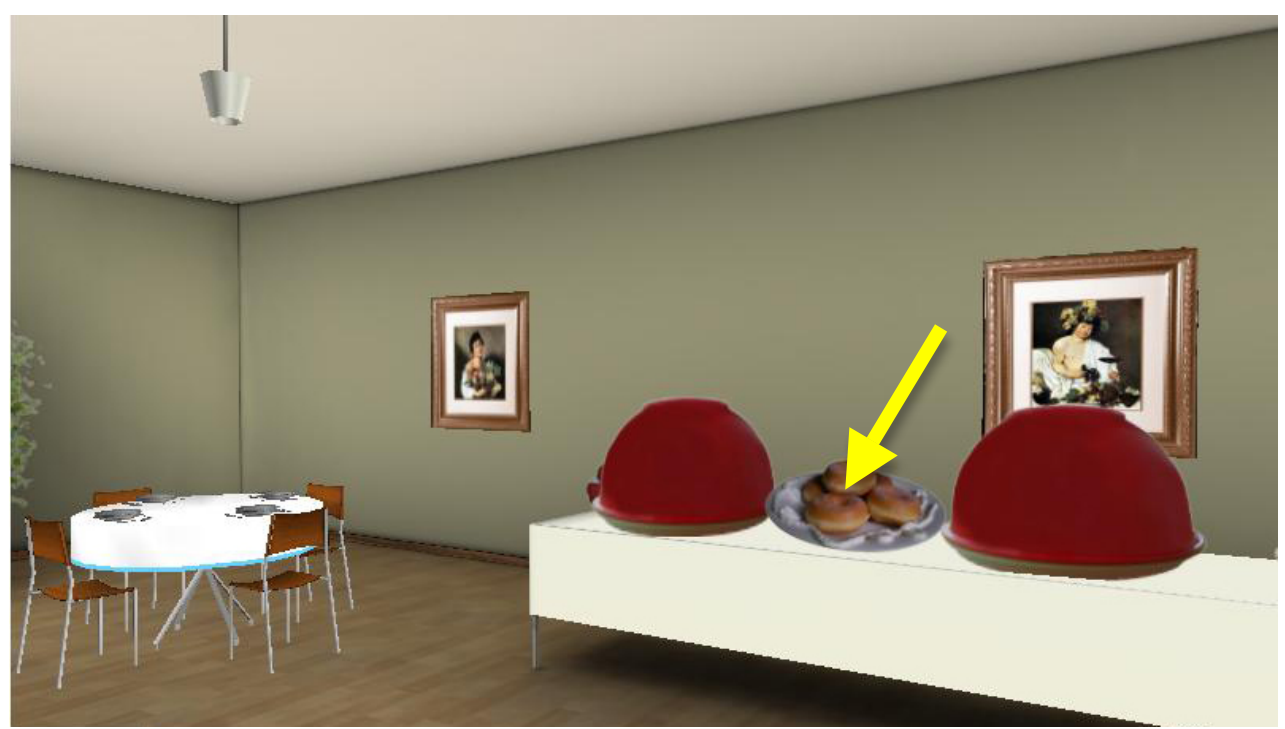

(b)

Fig. 2. The virtual restaurant (a) In the VR condition, subjects were asked to move around the room and to stand in front of the six plates covered by the red lids (the same used in the $\mathrm{RF}$ and $\mathrm{PH}$ conditions) indicated by the yellow arrow on the right side of the figure. (b) Standing in front of the plates, subjects were asked to select them one by one, to virtually remove the lid and to observe the food for $30 \mathrm{sec}$. After this time, the lid was automatically put back on the plate and the subject could do the second selection.

The order of presentation of each experimental condition, as well as the order of appearance of each food within the different conditions, was counterbalanced for each participant following a previously established randomization schema obtained from http://www.randomizer.org/.

All subjects were tested at least $2 \mathrm{~h}$ after a meal in order to avoid effects related to excessive hunger or overeating.

Before the RF and $\mathrm{PH}$ conditions there was a 3 min baseline during which subjects were asked to stay completely relaxed, while their physiological parameters were recorded. Because in the VR condition subjects used their right hand (all participants were right handed) to move inside the environment using a joystick, in order to control the hand movement, the baseline for the VR condition was recorded during a virtual navigation through an empty neutral space.

Once the physiological baselines were recorded, subjects were also asked to complete the STAI-S and the VAS-A. After that, the experimental session started, and heart rate, skin conductance and respiration rate were continuously recorded until the end of the task. Then, in order to measure 
the psychological variations occurred during the three different exposure conditions, subjects completed the STAI-S and the VAS-A again immediately after each session. The Presence Questionnaire was also administered at the end of the VR exposure. A pause of 5 min was planned between the sessions (Figure 3).

\begin{tabular}{|c|c|c|c|c|}
\hline $\begin{array}{l}\text { Food } 1 \\
(30 \mathrm{~s})\end{array}$ & $\begin{array}{l}\text { Pause } \\
(30 \mathrm{~s})\end{array}$ & $\begin{array}{l}\text { Food } 2 \\
(30 \mathrm{~s})\end{array}$ & $\begin{array}{l}\text { Pause } \\
(30 \mathrm{~s})\end{array}$ & $\begin{array}{l}\text { Food } 3 \\
(30 \mathrm{~s})\end{array}$ \\
\hline $\begin{array}{l}\text { Food } 4 \\
(30 s)\end{array}$ & $\begin{array}{l}\text { Pause } \\
(30 \mathrm{~s})\end{array}$ & $\begin{array}{l}\text { Food } 5 \\
(30 \mathrm{~s})\end{array}$ & $\begin{array}{l}\text { Pause } \\
(30 \mathrm{~s})\end{array}$ & $\begin{array}{l}\text { Food } 6 \\
(30 s)\end{array}$ \\
\hline
\end{tabular}

Questionnaires (STAI-S and VAS-A) + ITC-SOPI (only in the VR condition)

Five-minute rest

Fig. 3. Time-schedule of the experiment (repeated for all the three conditions).

\section{Statistical analysis}

Several within-subject repeated measure analysis of variance (ANOVA) tests were performed separately in each of the three groups of subjects to calculate the effects of exposure to the different kinds of food (real, photograph, and virtual) compared to the baseline. Then, the differences between each dependent variable measured after the exposure to food and the correspondent baseline were calculated. In the case of physiological measurements we calculated the differences between the mean values of $\mathrm{HR}$, SCR and RESP recorded during the exposure and the mean values obtained from the correspondent 3 min baselines. These values were used to conduct several $3 \times 3$ repeated measure ANOVA tests in order to test whether participants' psychological and physiological responses changed depending on the kind of exposure (real food, pictures of food or virtual 
food), and the group (AN, BN or CTR).

Finally, we calculated if symptoms severity, and the degree of presence experienced in the VR condition influenced the subjects' responses.

\section{Results}

Within-subject repeated measure ANOVA tests showed that exposure to real food, photographs of food and virtual food caused a significant increase in the STAI-S questionnaire, VAS-A, HR and SCR in both AN and BN patients, but not in the respiration rate, compared to the baseline. However, no differences were found between the baseline and the three experimental conditions in the CTR group (Table 3).

\begin{tabular}{|c|c|c|c|c|c|c|c|c|c|c|c|c|}
\hline & \multicolumn{9}{|c|}{ STAI-S } & \multicolumn{6}{c|}{ VAS-A } \\
\hline & \multicolumn{2}{|c|}{$R F$} & \multicolumn{2}{|c|}{$P H$} & \multicolumn{2}{|c|}{$V R$} & \multicolumn{2}{|c|}{$R F$} & \multicolumn{2}{c|}{$P H$} & \multicolumn{2}{c|}{$V R$} \\
\hline & $\mathrm{F}$ & $\mathrm{p}$ & $\mathrm{F}$ & $\mathrm{p}$ & $\mathrm{F}$ & $\mathrm{p}$ & $\mathrm{F}$ & $\mathrm{p}$ & $\mathrm{F}$ & $\mathrm{p}$ & $\mathrm{F}$ & $\mathrm{p}$ \\
\hline AN & 5.82 & 0.012 & 4.01 & 0.048 & 5.78 & 0.02 & 5.01 & 0.018 & 4.10 & 0.045 & 4.98 & 0.030 \\
\hline BN & 5.12 & 0.025 & 3.52 & 0.04 & 5.01 & 0.029 & 5.09 & 0.026 & 3.70 & 0.037 & 5.01 & 0.029 \\
\hline
\end{tabular}

(a)

\begin{tabular}{|c|c|c|c|c|c|c|c|c|c|c|c|c|}
\hline & \multicolumn{9}{|c|}{ HR } & \multicolumn{6}{c|}{ SCR } \\
\hline & \multicolumn{2}{|c|}{$R F$} & \multicolumn{2}{|c|}{$P H$} & \multicolumn{2}{|c|}{$V R$} & \multicolumn{2}{c|}{$R F$} & \multicolumn{2}{|c|}{$P H$} & \multicolumn{2}{c|}{$V R$} \\
\hline & $\mathrm{F}$ & $\mathrm{p}$ & $\mathrm{F}$ & $\mathrm{p}$ & $\mathrm{F}$ & $\mathrm{p}$ & $\mathrm{F}$ & $\mathrm{p}$ & $\mathrm{F}$ & $\mathrm{p}$ & $\mathrm{F}$ & $\mathrm{p}$ \\
\hline AN & 4.49 & 0.031 & 4.20 & 0.043 & 5.01 & 0.029 & 5.98 & 0.090 & 4.05 & 0.045 & 4.90 & 0.030 \\
\hline BN & 5.00 & 0.027 & 2.99 & 0.045 & 4.99 & 0.030 & 3.20 & 0.038 & 2.28 & 0.048 & 4.80 & 0.033 \\
\hline
\end{tabular}

(b)

Tab. 3. Within-subjects repeated-measure ANOVAs comparing the effects of different types of food presentation (RF, PH, VR) on psychological (a) and physiological (b) responses of $\mathrm{AN}, \mathrm{BN}$ and $\mathrm{CTR}$ subjects compared to the baseline (only significant values are reported).

Variations in psychological responses depending on the kind of exposure in patients and controls

Repeated measures ANOVA tests were conducted in order to test whether the responses to the STAI-S and the VAS-A changed depending on the presentation condition (RF, PH, VR), and the group (AN, BN or CTR).

Results regarding the STAI-S showed a significant effect of the variables 'condition' $\left(\mathrm{F}(2,54)=2.592 ; P<0.05\right.$; partial eta $\left.{ }^{2}=0.102\right)$ and 'group' (F $(2,27)=1.89 ; P<0.05$; partial eta $2=0.099)$, and a significant interaction between them $\left(\mathrm{F}(4,54)=2.986 ; P<0.05\right.$; partial eta $\left.{ }^{2}=0.087\right)$. Similar results were obtained analyzing the VAS-A scores: the effect of the 
variables condition $\left(\mathrm{F}(2,54)=3.097 ; P<0.05\right.$; partial eta $\left.{ }^{2}=0.089\right)$ and group $\left(\mathrm{F}(2,27)=1.98 ; P<0.05\right.$; partial eta $\left.^{2}=0.107\right)$, and the interaction between the variables condition and group were significant $(\mathrm{F}(4,54)=$ 1.85; $P<0.05$; partial eta ${ }^{2}=0.076$ ). Post hoc analysis and contrasts showed that both AN and BN groups experienced higher level of subjective anxiety compared to the CTR subjects $(P<0.001)$, and that they felt significantly more anxious when exposed to real and virtual food than when they were exposed to the pictures of food $(P<0.05)$. No significant differences were found between the STAI-S and the VAS-A values recorded during real and virtual exposure in the two groups of eating disorder patients. CTR subjects showed similar STAI-S and VAS-A scores in all conditions (Figures 4 and $5)$.

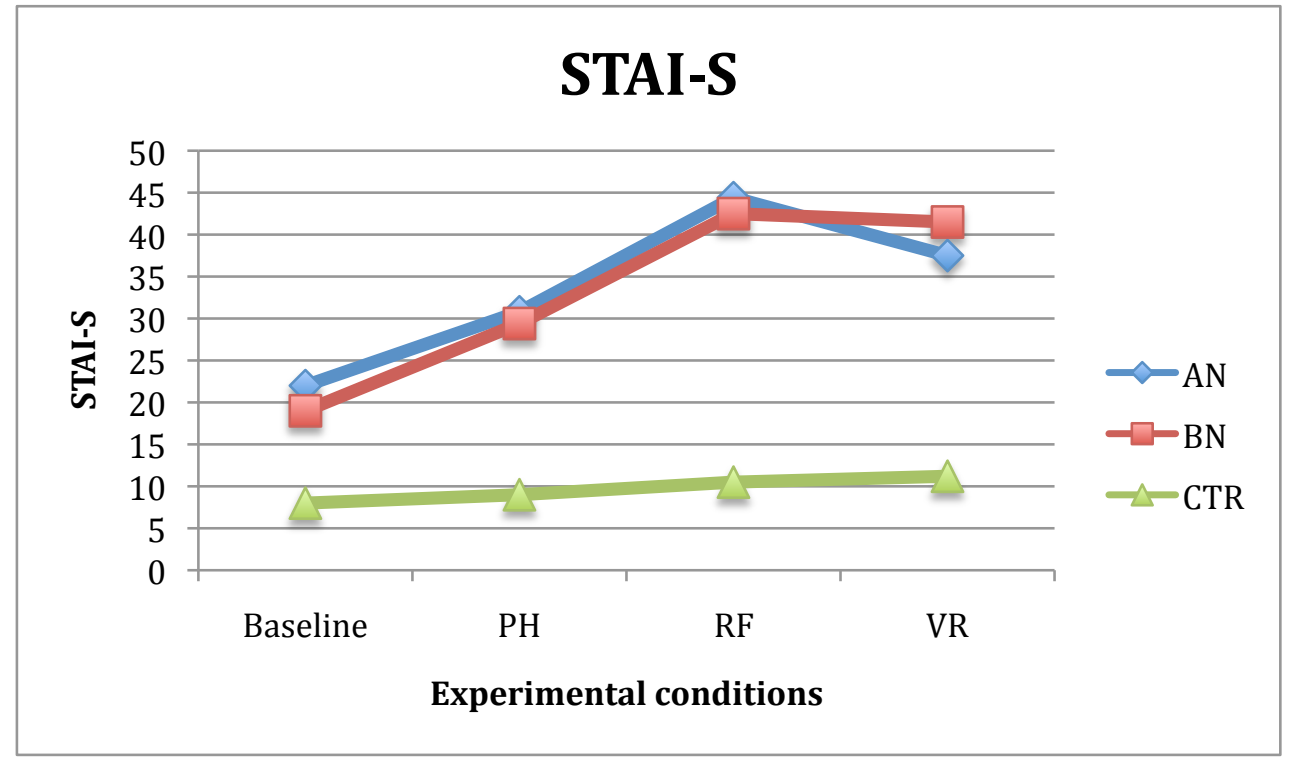

Fig. 4. STAI-S mean scores in AN, BN and CTR groups after the 3 different food exposures. 


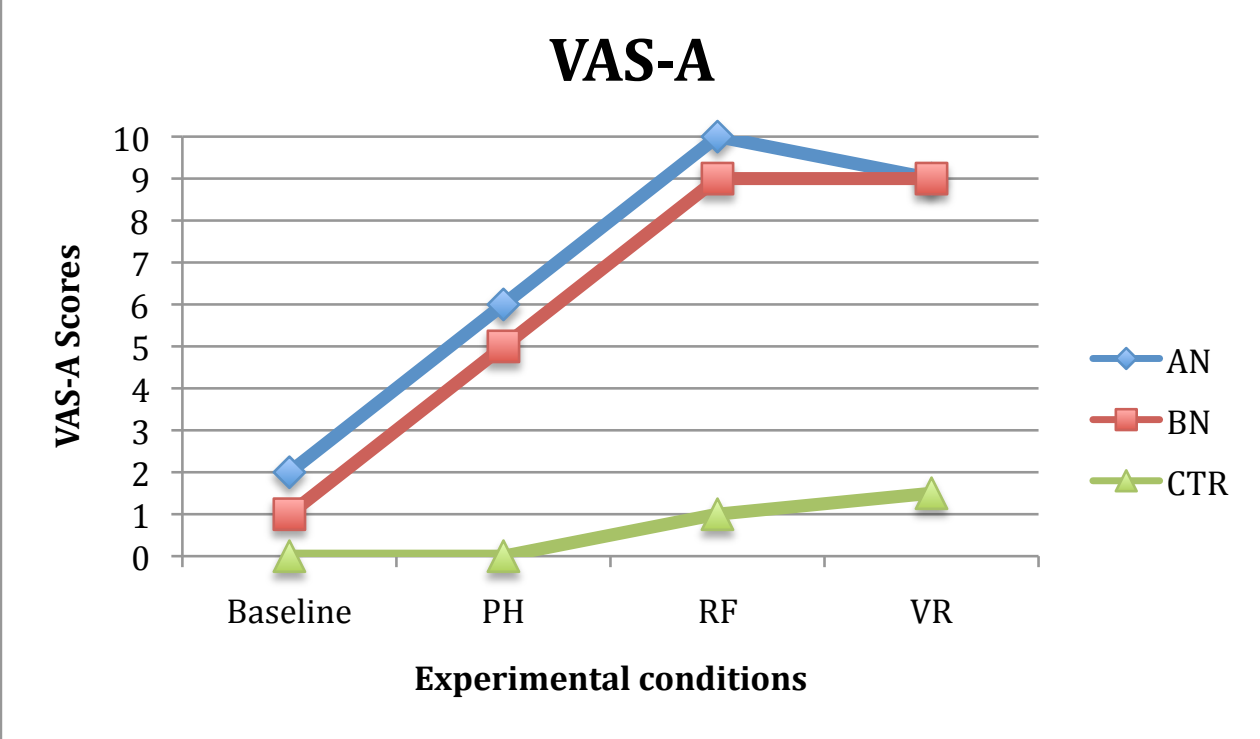

Fig. 5. VAS-A mean scores in $\mathrm{AN}, \mathrm{BN}$ and CTR groups after the 3 different food exposures.

Variations in physiological responses depending on the kind of exposure in patients and controls

Repeated measures ANOVA tests (3 (conditions) X (groups)) were also conducted in order to test whether HR, SCR, and RESP changed depending on the presentation condition (RF, PH, VR), and the group (AN, BN or CTR without ED).

Results regarding the HR showed a significant effect of the variables condition $\left(\mathrm{F}(2,54)=1.245 ; P<0.05 ;\right.$ partial eta $\left.^{2}=0.108\right)$ and group $(\mathrm{F}(2$, $27)=1.042 ; P<0.05 ;$ partial eta $\left.^{2}=0.112\right)$, and a significant interaction between them $\left(\mathrm{F}(4,54)=2.002 ; P<0.05\right.$; partial eta $\left.{ }^{2}=0.083\right)$. Similar results were also obtained analyzing the SCR values. Once again, the effect of the variables condition $\left(\mathrm{F}(2,54)=2.438 ; P<0.05\right.$; partial eta $\left.^{2}=0.065\right)$ and group $\left(\mathrm{F}(2,27)=1.98 ; P<0.05 ;\right.$ partial eta $\left.^{2}=0.086\right)$, and the interaction between the variables condition and group were significant (F (4, $54)=1.322 ; P<0.05$; partial eta $\left.{ }^{2}=0.075\right)$. Post hoc analysis and contrasts showed higher HR $(P<0.05)$ and SCR $(P<0.05)$ in AN and BN groups compared to CTR subjects. In both groups of patients, the level of physiological anxiety was higher in the RF and VR condition, than in the $\mathrm{PH}$ condition $(P<0.05)$. No significant differences were found between HR 
and SCR values recorded during real and virtual exposure in the two groups of eating disorder patients. CTR subjects showed similar scores in all conditions (Figures 6 and 7). No significant effects were found analyzing the RESP responses in any of the experimental group.

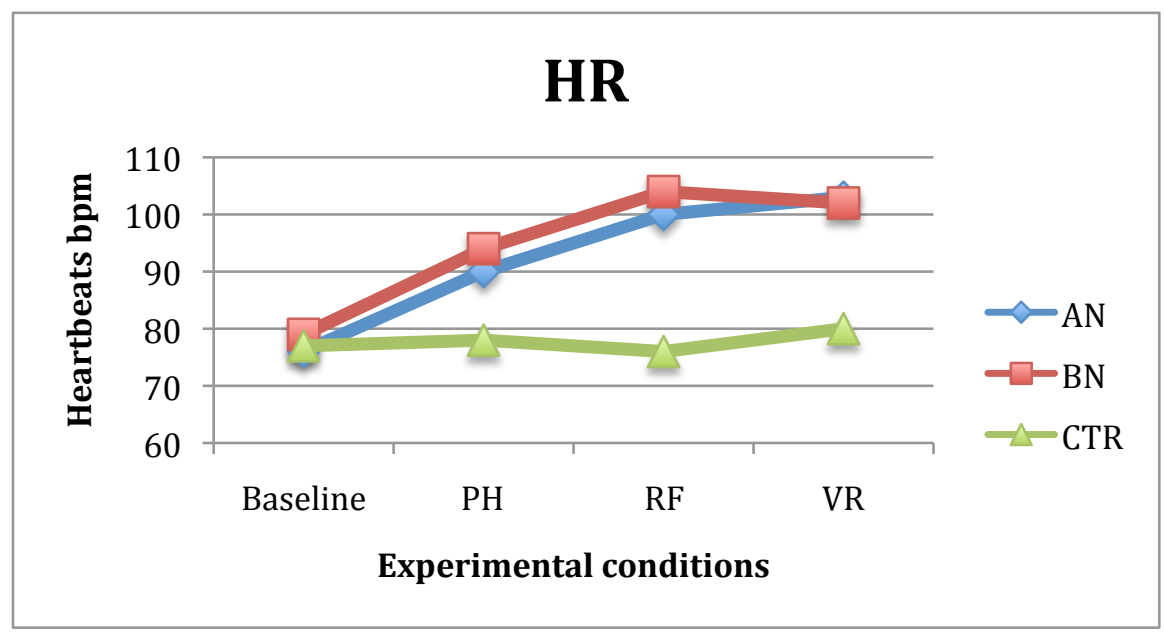

Fig. 6. HR mean scores in AN, BN and CTR groups recorded during the 3 different food exposures.

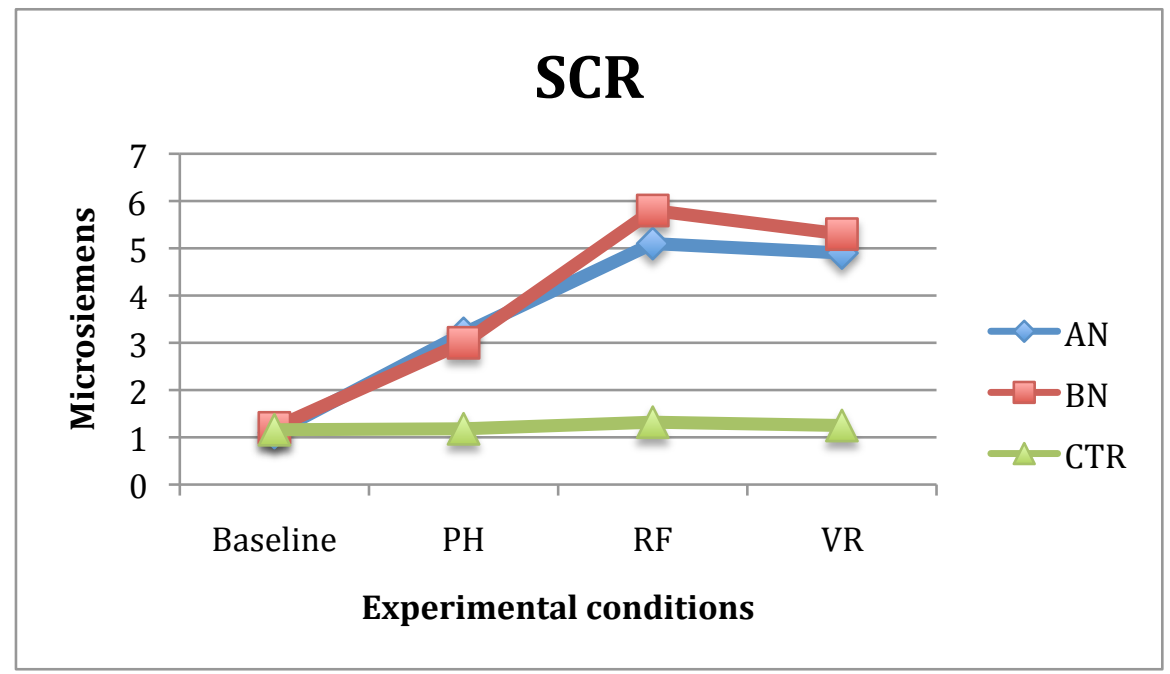

Fig. 7. SCR mean scores in AN, BN and CTR groups recorded during the 3 different food exposures. 
Finally, we investigated if the degree of presence experienced in the VR condition and measured with the ITC-SOPI questionnaire, and symptoms severity, assessed with the EDI-2 influenced the patients' emotional responses. As suggested by Gutierrez-Maldonado et al. [17], we divided the ED samples (AN and $\mathrm{BN}$ ) into quartiles and selected the first $(25 \%$ with the lowest scores on the ITC-SOPI) and the fourth $(25 \%$ with the highest scores). A simple effect of the degree of presence on the STAI-S $(F=2.80$, $P<0.05)$ and the VAS-A $(\mathrm{F}=2.51, P<0.05)$ was found. However, we did not find any significant effect of the EDI-2 score on patient emotional reactivity.

\section{Discussion}

This preliminary study was aimed at testing the theoretical assumption that a virtual experience elicits emotional responses comparable to those produced by real exposure. In addition, we also assumed that the sense of presence induced by the VR immersion makes the virtual experience more realistic, and consequently more effective than static pictures, in producing emotional responses in humans. In accordance with the first hypothesis, our data show that virtual food is as effective as real food, and more effective than photographs of food, in producing psychological and physiological responses in patients with ED, suggesting a possible advantage of using virtual stimuli instead of static pictures as an alternative to real stimuli to induce emotional reactions in subjects. This finding appears to be not specifically related to the diagnosis ( $\mathrm{AN}$ or $\mathrm{BN})$, as suggested by the fact that there were no significant differences in the emotional response recorded between the two groups of patients.

Not even the severity of illness seems to influence the patients' reactions, as subjects with a mild, moderate or severe eating disorder did not significantly differ in their emotional responses to real or virtual food. However, we did not find any significant variation in the controls' emotional reactions in any of the experimental conditions. This is not surprising because, as happens in real life, food does not represent a stressful stimulus for healthy people.

Regarding our second hypothesis, we found an effect of subjects' degree of presence experienced in the VR condition on their level of perceived anxiety (STAI-S and VAS-A): the higher the sense of presence, the higher the level of anxiety. The sense of presence in virtual reality is defined as 'the participant's sense of "being there" in the virtual environment' [4] and it is 
obtained through two factors: immersion and interaction. Immersion is provided by the use of technological devices such as HMDs that permit a 3D experience, while interaction is the possibility given to the users to interact in real time with the virtual environment. The higher the sense of presence, the more realistic the virtual experience, and more intense the emotional involvement.

Immersion and interaction are the key distinctive factors that make the difference between the VR and the PH conditions. In the latter, subjects can only passively observe static pictures, while in the VR condition they can actively explore the environment, approach the food and virtually touch it, as they would do in real-life situations. We argue that the effectiveness of virtual and real stimulations is the reason why both psychological (STAI-S, and VAS-A) and physiological (HR and SCR) responses appear to be consistently higher in the RF and VR than in the PH condition.

Thus, this result showing a similar pattern of psychological and physiological responses is rather new considering that, to date, there have been many studies that separately investigated psychological or physiological responses during VR exposure, but only few assessing the effects of stressors presented in a virtual environment on the subjective and objective response of anxiety [28, 29]. Regarding the general lack of significant variations on respiration, we hypothesize that it may be due to the fact that only respiration rate was assessed and not tidal volume, and anxiety mainly affects tidal volume rather than rate [30].

To date, despite the large amount of data demonstrating the efficacy of VRbased approaches for the treatment of different psychological disorders [2], none of the previous work had directly investigated if the exposure to virtual stimuli is able to elicit emotional reactions similar to those elicited by reallife exposure, which is the added value of using VR instead of simple static pictures. Even though it was accomplished on only two small samples of ED patients, these preliminary data encourage the use of VR in clinical (exposure therapy) and even non-clinical (task learning) settings in which a highly customizable and controllable stimulation is preferred to a real-life one. Additionally, our data emphasize the role of presence in the emotional processes, proving that, even if definitively more expensive, VR is preferable to static images for generating affective responses in humans. So, in accord with the previous studies [17, 18], the present research adds some evidence that virtual stimuli can be used instead of the real ones to elicit patients' emotions.

Despite the clearness of the present findings, this study has some important limitations. First, the small number of subjects per group makes us cautious 
about the generalization of the results. A future randomized controlled study including a larger sample will address this issue. Second, in the VR condition subjects were exposed to virtual food in a virtual restaurant, while in the other two conditions they were exposed to food only. A restaurant is a broader stimulus than food because it elicits a complex context possibly inducing a greater level of anxiety than food alone, and also other fears, not strictly or necessarily related to food (for example, agoraphobia). In order to control this aspect in future studies, virtual food could be presented in neutral virtual environments not specifically related to eating contexts. Thus, even if considered a limitation in the present study, the possibility to measure subjects' reactions in a complex virtual environment is a great advantage offered by virtual reality, with poor feasibility for testing the subjects' responses in a real complex environment such as a restaurant.

\section{Conclusions}

In conclusion, though preliminary, the present data show that virtual stimuli are as effective as real ones, and more effective than static pictures, in generating emotional responses in ED patients. Unlike exposure to photographs, in vivo exposure and guided imagination, VR offers a good ecological validity, and also a fair internal validity, while allowing strict control over the variables. More generally, the present results provide initial evidence of the potential of VR in a variety of experimental, training and clinical contexts, its range of possibilities being extremely wide and customizable. In particular, in a therapeutic perspective based on a cognitive behavioral approach, the use of VR instead of real stimuli facilitates the provision of very specific contexts to help patients to cope with their conditions through a very controlled stimulation. At the same time, the results of the present study indicate that even very low cost VR software like NeuroVR can be used to screen, evaluate, and eventually treat the emotional reactions provoked by specific stimuli in patients affected by psychological conditions. 
Chapter III

\section{Acknowledgements}

The authors would like to thank Professor Laura Bellodi from Università

Vita-Salute San Raffaele (Milan, Italy) for her help in the protocol preparation. 


\section{References}

1. Pull CB: Recent trends in the study of specific phobias. Curr Opin Psychiatry 2008, 21:43-50.

2. Gorini A, Riva G: Virtual reality in anxiety disorders: the past and the future. Expert Rev Neurother 2008, 8:215-233.

3. Gregg L, Tarrier N: Virtual reality in mental health: a review of the literature. Soc Psychiatry Psychiatr Epidemiol 2007, 42:343-354.

4. Barfield W, Zeltzer D, Sheridan TB, Slater M: Virtual environments and advance interface design. In Presence and performance within virtual environments. Oxford, UK: Oxford University Press; 1995: 473-541.

5. Lombard M, Ditton T: At the heart of it all: the concept of presence. J Comp Mediat Comm, 1997, 3.

6. Slater M, Steed A, McCarthy J, Maringelli F: The influence of body movement on subjective presence in virtual environments. Human Factors 1998, 40:469-477.

7. Botella C, Quero S, Banos RM, Perpina C, Garcia Palacios A, Riva G: Virtual reality and psychotherapy. Stud Health Technol Inform 2004, 99:37-54.

8. Vincelli F: From imagination to virtual reality: the future of clinical psychology. Cyberpsychol Behav 1999, 2:241-248.

9. Bordnick PS, Graap KM, Copp H, Brooks J, Ferrer M, Logue B: Utilizing virtual reality to standardize nicotine craving research: a pilot study. Addict Behav 2004, 29:1889-1894.

10. Slater M, Pertaub DP, Barker C, Clark DM: An experimental study on fear of public speaking using a virtual environment. Cyberpsychol Behav 2006, 9:627-633.

11. Carter FA, Bulik CM, McIntosh VV, Joyce PR: Cue reactivity as a predictor of outcome with bulimia nervosa. Int J Eat Disord 2002, 31:240-250.

12. Toro J, Cervera M, Feliu MH, Garriga N, Jou M, Martinez E, Toro $\mathrm{E}$ : Cue exposure in the treatment of resistant bulimia nervosa. Int $\mathrm{J}$ Eat Disord 2003, 34:227-234.

13. Gordon CM, Dougherty DD, Fischman AJ, Emans SJ, Grace E, Lamm R, Alpert NM, Majzoub JA, Rauch SL: Neural substrates of anorexia nervosa: a behavioral challenge study with positron emission tomography. J Pediatr 2001, 139:51-57.

14. Legenbauer T, Vogele C, Ruddel H: Anticipatory effects of food 
exposure in women diagnosed with bulimia nervosa. Appetite 2004, 42:33-40.

15. Nederkoorn C, Guerrieri R, Havermans RC, Roefs A, Jansen A: The interactive effect of hunger and impulsivity on food intake and purchase in a virtual supermarket. Int J Obes (Lond) 2009, 33:905912.

16. Lozano JA, Alcaniz M, Gil JA, Moserrat C, Juan MC, Grau V, Varvaro H: Virtual food in virtual environments for the treatment of eating disorders. Stud Health Technol Inform 2002, 85:268-273.

17. Gutierrez-Maldonado J, Ferrer-Garcia M, Caqueo-Urizar A, LetosaPorta A: Assessment of emotional reactivity produced by exposure to virtual environments in patients with eating disorders. Cyberpsychol Behav 2006, 9:507-513.

18. Ferrer-Garcia M, Gutierrez-Maldonado J, Caqueo-Urizar A, Moreno E: The validity of virtual environments for eliciting emotional responses in patients with eating disorders and in controls. Behav Mod 2009, 33:830-854.

19. Sheehan DV, Lecrubier Y, Sheehan KH, Amorim P, Janavs J, Weiller E, Hergueta T, Baker R, Dunbar GC: The Mini-International Neuropsychiatric Interview (M.I.N.I.): the development and validation of a structured diagnostic psychiatric interview for DSMIV and ICD-10. J Clin Psychiatry 1998, 59:22-33.

20. Garner DM: Eating Disorder Inventory-II. Adattamento italiano a cura di Rizzardi, M., Trombini E., Trombini, G. Firenze, Italy: Organizzazioni Speciali; 1995.

21. Spielberger CD, Gorsuch RL, Lushene RE: Manual for the state-trait anxiety inventory. Palo Alto, CA, USA: Consulting Psychologists Press; 1970.

22. Gross J, Levenson RW: Emotion elicitation using films. Cognition Emotion 1995, 9:87-108.

23. Abu-Saad H, Holzemer WL: Measuring children's selfassessment of pain. Iss Comp Pediatr Nurs 1981, 5:337-349.

24. Price DD, McGrath PA, Rafii A: The validation of visual analogue scales as ratio scale measures for chronic and experimental pain. Pain 1983, 17:45-56.

25. Reading AE: A comparison of pain rating scales. J Psychosom Res 1980, 24:119-124.

26. Lessiter J, Freeman J, Keogh E, Davidoff JD: A cross-media presence questionnaire: the ITC sense of presence inventory. Presence Teleoperators Virtual Environments 2001, 10:282-297. 
27. Riva G, Gaggioli A, Villani D, Preziosa A, Morganti F, Corsi R, Faletti G, Vezzadini L: NeuroVR: an open source virtual reality platform for clinical psychology and behavioral neurosciences. Stud Health Technol Inform 2007, 125:394-399.

28. Côté S, Bouchard S: Documenting the efficacy of virtual reality exposure with psychophysiological and information processing measures. Appl Psychophysiol Biofeedback 2005, 30:217-232.

29. Walshe D, Lewis E, O'Sullivan K, Kim SI: Virtually driving: are the driving environments "real enough" for exposure therapy with accident victims? An explorative study. Cyberpsychol Behav 2005, 8:532-537.

30. Martinez JM, Papp LA, Coplan JD, Anderson DE, Mueller CM, Klein DF, Gorman JM: Ambulatory monitoring of respiration in anxiety. Anxiety 1996, 2:296-302. 



\section{CHAPTER IV}

\section{Nonhomogeneous results in place learning among panic disorder patients with agoraphobia}

A. Gorini, K. Schruers, G. Riva, E. Griez.

Psychiatry Research. 2010, May 28. 



\section{Abstract}

Patients affected by panic disorder with agoraphobia (PDA) often suffer of visuo-spatial disturbances. In the present study, we tested the place learning abilities in a sample of 31 PDA patients compared to 31 healthy controls (CTR) using the ComputerGenerated Arena (C-G arena), a desktop-based computer program developed at the University of Arizona (Jacobs et al 1997, for further detail about the program, see http://web.arizona.edu/ arg/data.html). Subjects were asked to search the computer-generated space, over several trials, for the location of a hidden target. Results showed that control subjects rapidly learned to locate the invisible target and consistently returned to it, while PDA patients were divided in two subgroups: some of them (PDA-A) were as good as controls in place learning, while some others (PDA-B) were unable to learn the correct strategies to find the target. Further analyses revealed that PDA-A patients were significantly younger and affected by panic disorder from less time than PDA-B, indicating that age and duration of illness can be critical factors that influence the place learning abilities. The existence of two different subgroups of PDA patients who differ in their spatial orientation abilities could provide new insight into the mechanisms of panic and open new perspectives in the cognitive-behavioural treatment of this diffuse and disabling disorder. 


\section{Introduction}

Anxiety disorders are often characterized by automatic attentional biases for selective processing of information or stimulation related to cues perceived as threatening in the anxious people's environment (Keogh and French, 1999; Wittchen and Hoyer, 2001). In particular, visuo-spatial cognition biases are common in patients affected by panic disorder with agoraphobia (PDA), who are often so worried about their own physical reactions that they become unable to be attentive to changes occurring in the surrounding environment (Kallai et al., 1995). These observations are supported by different experimental studies showing that patients with PDA have scarce abilities in orientating in a maze and in performing blind orientation tasks as compared with patients affected by other anxiety disorders and healthy controls (Kallai et al., 1995; Kallai et al., 1996). Recent data obtained by Kallai et al. (Kallai et al., 2007a) have also shown a correlation between altered physiological parameters and the PDA patients' inability to detect the navigation signals indicating the right route to exit from a labyrinth.

Disorders of visuo-spatial attention have been found in PDA patients during a computerized visual target discrimination task (Dupont et al., 2000) and in a distance estimation task, indicating the presence of a possible distortion in the patients' representational mechanisms of the extrapersonal space (Iavarone et al., 2005). PDA patients also present deficit in spatial memory and learning, as shown by Boldrini et al. (Boldrini et al., 2005). Moreover, the positive effects of an attentional fixation training in reducing panic related symptoms (Kallai et al., 1999) strengthen the hypothesis that spatial disturbances are often associated with PDA.

Besides these experimental data supporting the hypothesis that panic disorder with agoraphobia should at least partially depend on the ways in which the cognitive structures interact with the situational variables (Taylor et al., 1986), there are a series of neuropsychological studies that have failed to find any spatial deficit in PDA patients compared to control subjects (Gladsjo et al., 1998; Purcell et al., 1998). These discordant results make the role of visuo-spatial abilities in PDA still unclear. However, as shown by Kallai and coll. (Kallai et al., 1999), understanding the role of orientation abilities in PDA is crucial to gain new insight into the mechanisms of panic disorder and to find an efficient therapeutic approach.

In the present study, we propose to test spatial orientation and place learning abilities in a sample of severe PDA patients using the Computer-Generated Arena (CGA), a desktop-based computer program developed at the 
University of Arizona ((Jacobs et al., 1997; Jacobs et al., 1998); for further detail about the program, see http://web.arizona.edu/ arg/data.html) representing a virtual adaptation of the original water maze task (Morris, 1981). The main advantage of using a virtual space instead of a real one, is the possibility to test patients in a safe and controlled environment reducing the risk, usually associated with in-vivo exposure, of inducing panic related symptoms and altering their physiological, emotional and cognitive functioning during the experimental session. Moreover, compared to the traditional neuropsychological tests, a virtual space has the advantage of enhancing the ecological validity of the test, increasing predictions about the patient' s functioning in the real world (Parsons et al., 2008). Up to date, several researches using such technology have been conducted (Gillner and Mallot, 1998; Jacobs et al., 1997; Jacobs et al., 1998; May et al., 1995; Nadel et al., 1998; Ruddle et al., 1997). The main findings emerged from these studies are that: (a) subjects can make accurate judgments about metrics in real space after learning in a virtual environment (Péruch et al., 1995), (b) there is good transfer of spatial information from virtual to real environments (Wilson et al., 1997), (c) different spatial performances in the virtual spaces are predicted by different search strategies that reproduce the strategies used in real spaces (Kallai et al., 2007b; Kallai et al., 2005), (d) virtual environments are suitable to explore the neural substrate of place learning and spatial navigation in humans (Thomas et al., 2001), and (e) virtual environments, and virtual reality technology in general, show promise in aiding neuropsychological evaluation and rehabilitation (Rizzo et al., 1998; Rose et al., 1996; Thomas et al., 2001). Additionally, thanks to their programmable flexibility, data-handling capabilities, and their psychometric properties, virtual environments reproducing classical navigation tasks have been also used to explore the issue of gender (Astur et al., 1998; Astur et al., 2004) and age-related (Thomas et al., 1999) differences showing robust sex differences in virtual place learning, as well as the presence of age-related changes in the human cognitive mapping system.

The C-G Arena consists in a computer-generated three-dimensional virtual space in which subjects are asked to find a hidden platform using a number of distal cues on the walls. This kind of place learning task requires distal spatial orientation abilities (Morris, 1981): to complete it subjects use only localized distal cues coming from fixed places at some distance from the target objects, learning and remembering location of the target relative to them. In order to successfully perform the task, organisms use a spatial map consisting of information about specific objects and relations among them, 
formed when they enter and observe a new environment for the first times (Jacobs et al., 1997). As demonstrated by Jacobs and coll. (Jacobs et al., 1998), the place learning in C-G space is comparable to both rat and human place learning in real space.

Using the C-G Arena we wanted to investigate if place learning based on distal cues occurs in PDA patients as it occurs in healthy subjects and if it generalizes from familiar to novel start locations. To answer these questions we used a version of the C-G Arena in which only distal cues existed and trained participants to find an invisible target entering in the virtual space from different start locations. Our hypothesis is that the ecological characteristics of the C-G Arena could be useful to discriminate the spatial abilities of subjects, eventually indicating difference between PDA patients and healthy controls, or within the PDA group itself, allowing the therapist to decide to integrate the traditional therapeutic approach with a spatial orientation training.

\section{Methods}

\section{Subjects}

Thirty-one patients with panic disorder and agoraphobia ( 7 males and 24 females; mean age: 35.52 years, S.D. $=14.30$; years of education: 16.54 , S.D. = 3.32) who applied for the cognitive-behavioral therapy (CBT) program at the Academic Anxiety Center (AAC) in Maastricht, NL, were included in the study. The mean duration of PDA was 8.77 years, S.D. = 8.28 years. Fifteen out of 31 patients who took psychotropic medications were asked to suspend them at least one week before their participation to the study (two weeks in case of antidepressant treatments). Psychiatric diagnosis was made according to the DSM-IV TR criteria by two experienced psychiatrists working at the AAC and not directly involved in the study. The Mini International Neuropsychiatric Interview (MINI) (Sheehan et al., 1998) was administered to support the diagnosis. Patients who received a different primary psychiatric diagnosis or affected by neurological illnesses that would interfere with completing the computerbased spatial task were excluded from the study. PDA was also investigated using the Panic and Agoraphobia Scale (P \& A) (Bandelow, 1995) (mean value $=25.87$, S.D. $=4.42)$ and the Agoraphobic Cognitions Questionnaire $($ ACQ) (Chambless et al., 1984) (mean value $=21.48$, S.D. $=13.45)$. 
In addition, 31 healthy volunteers (CTR), matched with patients on gender, age and educational level (12 males and 19 females; mean age: 30.23 years, S.D. $=12.02$; years of education: 14.13 , S.D. $=4.87$ ) and recruited by advertisement in local newspapers, were included in the study. They were also evaluated with the MINI in order to exclude any current or past psychiatric illness.

Participants meeting the inclusion criteria and having agreed in signing the informed consent were informed in advance about the aims and procedures of the experiment and recruited for the study.

In order to anticipate a possible distortion coming from individual differences of computer game playing practice (Waller, 2000) participants were pre-selected, during recruitment, on the basis of a questionnaire about their computer using habits. Only those subjects whose computer game playing did not exceed a half an hour per week were eligible for the study. Following the administration of this questionnaire, 3 healthy controls were excluded from the study.

\section{The C-G Arena software}

The Water Maze Task (Morris, 1981), a place-learning test originally developed by Morris to investigate the spatial abilities in rats, served as a model for developing the C-G Arena, a desktop-based computer-generated virtual space created to investigate the place learning abilities in humans (Jacobs et al., 1997). The C-G Arena is a three-dimensional circular virtual environment housed within a large experimental room (arena). The subjects' task is to explore it using a joystick, in order to find a platform (target) hidden on the floor. The experimental room consists of a computergenerated display of a 1500 X 1500 X 475 unit room (10 units corresponds to 1 virtual meter). The ceiling of the room is a light gray and the floor a dark gray. The walls of the room, programmed to appear at some distance from the arena wall, are arbitrarily designated the North, East, South, and West walls. The North wall is gray and displays a door flanked by two windows; the East wall displays six and one half arches; the South wall is gray and displays three centered windows; the West wall displays red bricks. A featureless purple wall, 460 units in radius and 30 units high, encloses the central portion of the floor of the experimental room, defining the arena (see Fig. 1). The actual viewpoint of the participants is a firstperson perspective, so they look at the scene as though standing on the floor of the arena.

The hidden target is a $142 \times 142$-unit square located on the floor of the experimental room. Its color is identical to the surrounding arena floor, but 
becomes red when subjects reach it and stand on it. Finally, a beep sounds each time the subject moves on it. The target is level with the arena floor. Another room of the same shape and size of the experimental room, but without any texture on the walls serves as training room (also called waiting room) and is located immediately before the entrance of the experimental room. No target is contained in it.

The arena is divided into four imaginary quadrants. Moving clockwise, the first is named Northwest (NW), the second is named Northeast (NE), the third is named Southeast (SE) and the fourth is named Southwest (SW). Lines delineating the quadrants are not visible. The invisible target is located in the NE quadrant for the entire duration of the experiment.

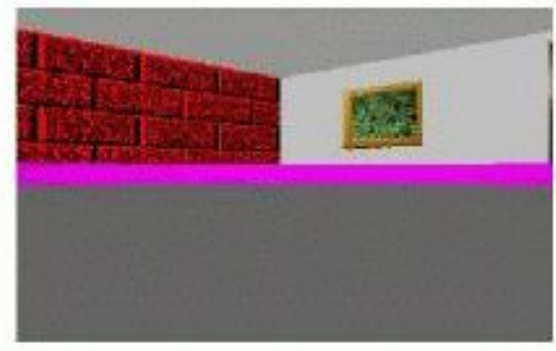

Northwest Quadrant

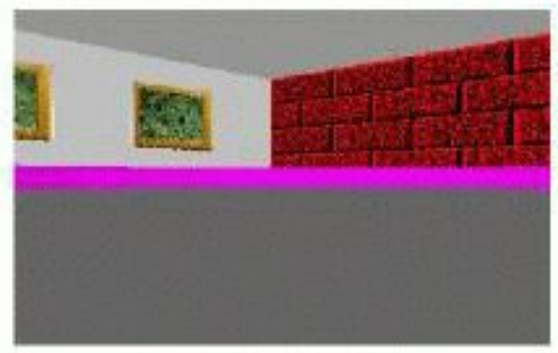

Southwest Quadrant

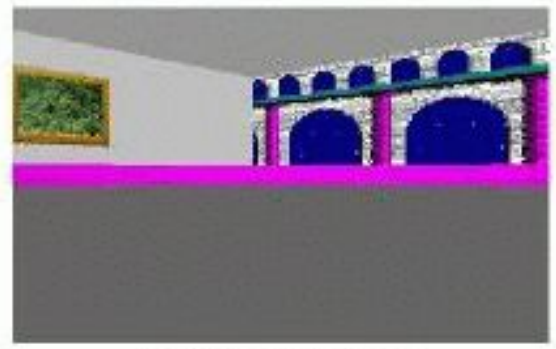

Northeast Quadrant

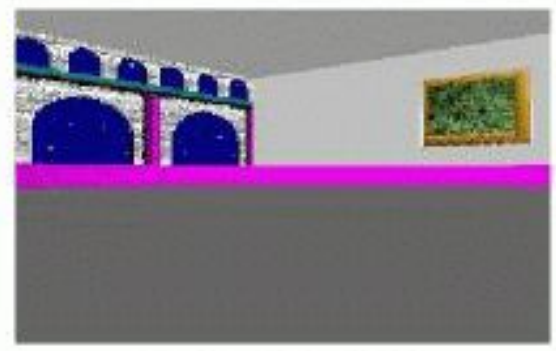

Southeast Quadrant

Fig. 1. Four screenshots of the virtual C-G arena showing the patterns placed on the room walls (the distal cues) as seen from inside the arena. Source:

http://web.arizona.edu/ arg/data.html.

\section{Procedure}

Participants were seated in front of a standard PC with a 17 in. SVGA screen, equipped with stereo speakers and a joystick. Each experimental session started in the waiting room, in which subjects became familiar with the virtual space and practiced virtual locomotion using the joystick. They 
could stay in this room all the time they needed. When ready, they were asked to press the space bar on the computer keyboard to be moved ("teleported") to the experimental room. At each trial subjects entered in the experimental room from a different starting position (randomly determined by the computer) facing and within 2 units of the arena wall, and were asked to turn around, search for, find and stand on the hidden platform located on the arena floor under the shortest time possible. The invisible target was centered in the NE quadrant, approximately 234 units from the closest part of the arena wall, in each acquisition trial. Once subjects found and stood on it, the target became visible and they had as much time as they wanted to stay on it trying to remember its position, using the distal cues on the walls. The position of the distal cues and the target remained the same during all the trials.

Subjects had a maximum of 4 minutes to find the platform and complete each trial. If they failed, the trial terminated and they were automatically teleported in the waiting room.

The entire experiment consisted in nine trials (acquisition trials) plus one (probe trial). The last probe trial was identical to the others except for the following: the target, unknown to the participant, was removed from the arena. Under these experimental conditions, appropriate performance requires sophisticated spatial computations involving the learned (and remembered) location of the object relative to the different distal cues.

The C-G arena software collected quantitative and qualitative data of the subjects' navigations. Quantitative data consisted in: path length and latency to find the target; time spent on the target during the acquisition trials; and time spent in the appropriate quadrant relative to the distal cues during the probe trial. Qualitative data consisted in path maps representing the subjects' spatial navigation strategies. In these bitmap images the arena is showed in a plan-view, divided into four quadrants, while the navigation path is drawn as a continuous line. The target platform is included in the NE quadrant.

All the participants completed the experimental procedures within $30-60$ minutes. 


\begin{tabular}{ll}
\hline $\begin{array}{l}\text { Parameter } \\
\text { Acquisition phase }\end{array}$ & \\
Number of trials & 9 \\
Experimental room start location & Randomized \\
Target condition & Invisible \\
Target location & NE quadrant \\
Time limit & $240 \mathrm{sec}$ \\
\hline Probe trial & \\
Number of trials & 1 \\
Experimental room start location & Randomized \\
Target condition & Absent \\
Time limit & $240 \mathrm{sec}$ \\
\hline
\end{tabular}

Tab. 1. Trial parameters for the C-G arena.

Psychological assessment

In order to verify their level of anxiety immediately before the test, participants were asked to fill out the $0-100$ visual analogue scale for anxiety (VAS-A). No significant differences were found in the subjective anxiety perceived immediately before the experiment between PDA (mean $=15.68$, S.D. $=7.26)$ and CTR subjects $($ mean $=13.90$, S.D. $=4.91),(\mathrm{t}=-$ $1.126(60), \mathrm{p}=0.265)$.

\section{Data analyses}

The $\mathrm{C}-\mathrm{G}$ arena software generates two separate data files. The first one contains information about: (1) latency, that is the time required to find the target; (2) path length, the distance travelled from the start point to the target; (3) dwell time, the time spent in each of the arena quadrant during the probe trial looking for the target; and (4) time spent on the target, used by subjects to learn the target position in relation to the distal cues. The second data file contains a pixel-by-pixel recording of the participant's experience in the arena. From this data file it is possible to generate an image of the search path taken on each trial. Latency, path length, dwell time, and time spent of the target were the dependent variables of our study. The Type I error rate $(\alpha)$ was set at 0.05 for all statistical decisions.

\section{Results}

All subjects involved in the study completed the computer task. Because in the first trial they had to guess where the target was, we decided to include 
in the analyses only data obtained from trial 2 to trial 9, while the last trial (probe trial), that did not contain the target, was analyzed separately.

\section{Place learning}

From trial 2 to trial 9, CTR subjects located the target more often than PDA patients did. On average, the CTR subjects located the target 7.6 times during the series of 8 acquisition trials, whereas PDA patients located it 6.8 times. The difference between these means is statistically significant $(\mathrm{t}=-$ $2.61(60), \mathrm{p}<0.05)$.

\section{Latency}

Figure 2 illustrates the mean time required to locate the invisible target during the place learning task for the PDA and the CTR groups. A split-plot analysis of variance (ANOVA) conducted on these data detected a significant main effect of group $(\mathrm{F}(1,60)=4,82)$, a significant main effect of trials $(F(7,60)=2.06)$, and no significant trials $x$ group interaction effect. Separate within-groups repeated measures ANOVAs detected no significant main effect of trials for the PDA group, but a significant main effect of trials for the CTR group $(\mathrm{F}(7,30)=2.46)$. Orthogonal post-hoc comparisons conducted on the data obtained from the CTR group showed the time they required to locate the invisible target decreased from the first to the last acquisition trial. 


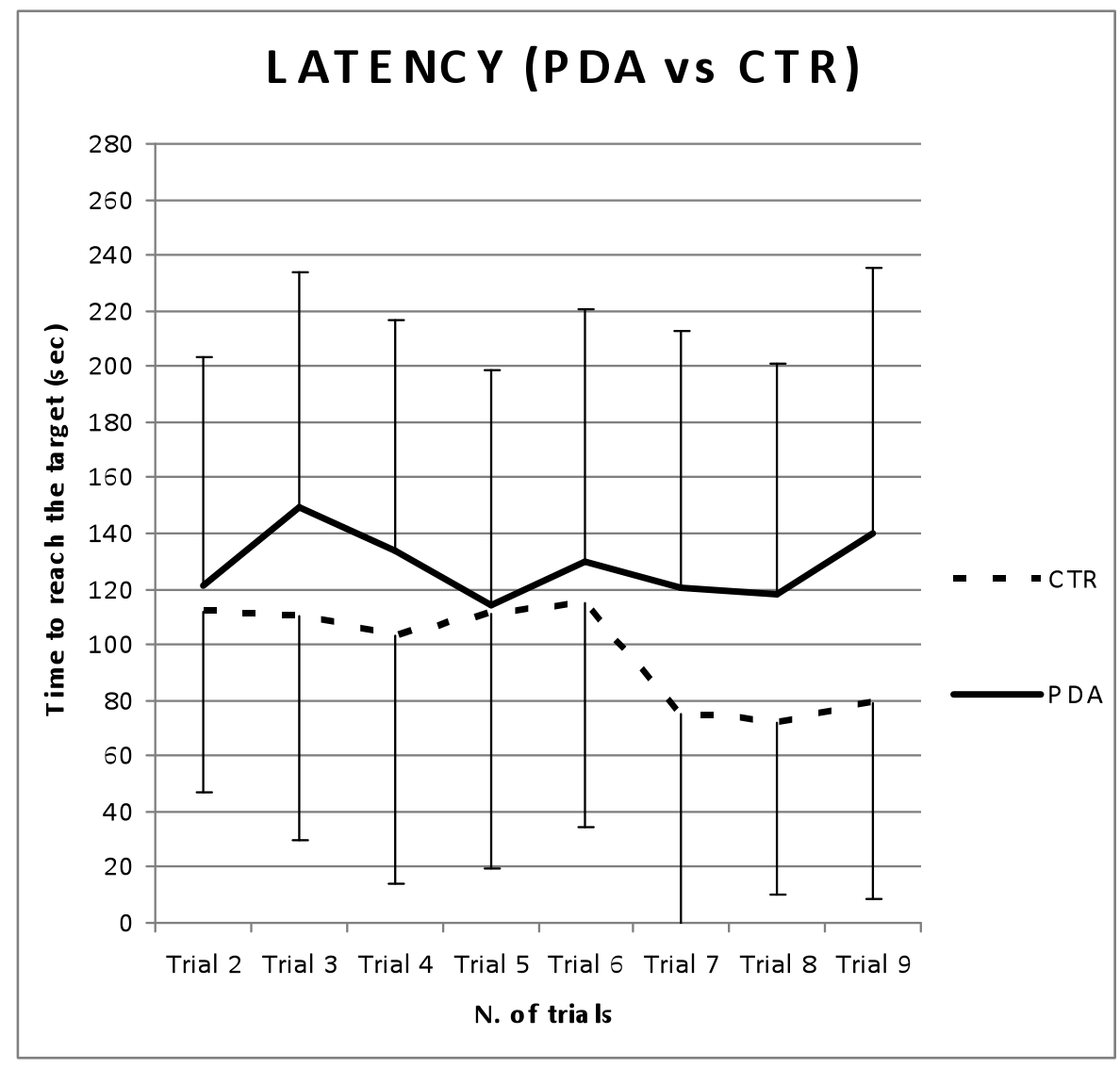

Fig. 2. The time in seconds (means and standard deviations of the mean) the 2 groups of participants required to locate the invisible target on each of the 8 acquisition trials.

\section{Path length}

Figure 3 illustrates the mean path length to the invisible target during the place learning task for the PDA and CTR groups. A split-plot ANOVA conducted on these data detected a significant main effect of group $(\mathrm{F}(1,60)$ $=8.50)$, no significant main effect of trials, and a significant trials $\mathrm{x}$ group interaction effect $(F(7,60)=1.98)$. Separate within-groups repeated measures ANOVAs detected no significant main effect of trials for the PDA group, but a significant main effect of trials for the CTR group $(\mathrm{F}(7,30)=$ 4.85). Orthogonal post-hoc comparisons conducted on the data obtained from the CTR group showed the path length they took to the invisible target decreased from the first to the last acquisition trial. 


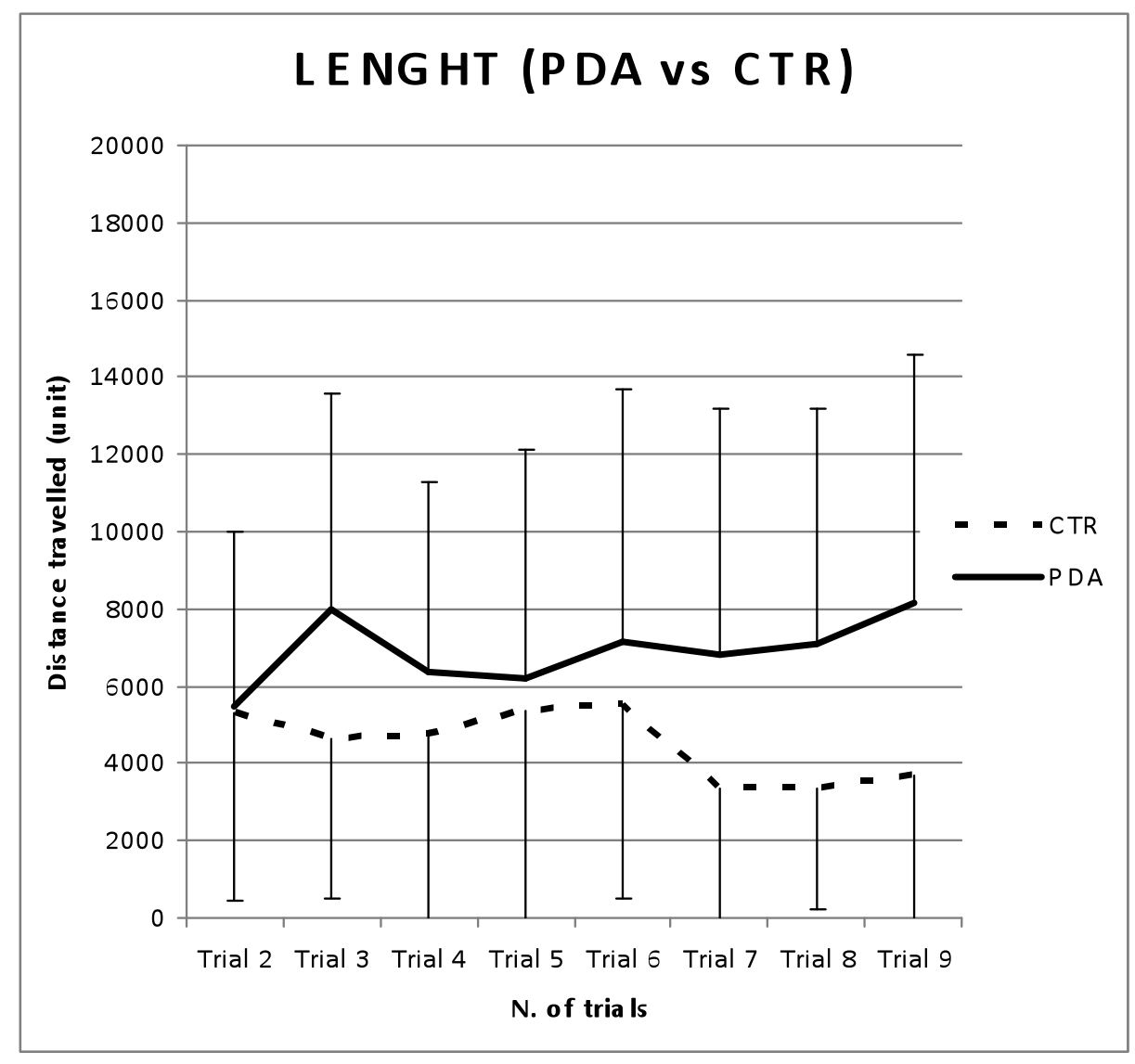

Fig. 3. The path length in units (means and standard deviations of the mean) the 2 groups of participants required to locate the invisible target on each of the 8 acquisition trials.

Dwell time (probe trial)

Figure 4 illustrates the mean time participants in each group spent searching each quadrant during the probe trial. Two independent within-subjects repeated measures ANOVAs detected a significant main effect of quadrant in the PDA group $(\mathrm{F}(3,30)=10.03)$, and a significant main effect of quadrant in the CTR group $(\mathrm{F}(3,30)=11.43)$. In particular, orthogonal posthoc contrasts conducted on the data obtained from the PDA patients revealed: (a) no significant difference in mean dwell time between the NE and SE quadrants, (b) a significant difference in mean dwell time between the NE and NW quadrants, and (c) a significant difference in mean dwell time between the NE and SW (mean NE $=84.41$, S.D. $=42.91$; mean $\mathrm{SE}=$ 72.31 , S.D. $=37.96$; mean $\mathrm{NW}=41.24$, S.D. $=28.85$; mean $\mathrm{SW}=36.84$, 
S.D. $=33.56)$. On the contrary, orthogonal post-hoc contrasts conducted on the data obtained from the CTR subjects revealed: (a) a significant difference in mean dwell time between the NE and SE quadrants, (b) a significant difference in mean dwell time between the NE and NW quadrants, and (c) a significant difference in mean dwell time between the $\mathrm{NE}$ and $\mathrm{SW}$ (mean NE $=$ 93.56, S.D. $=$ 50.94; mean $\mathrm{SE}=49.18$, S.D. $=$ 35.40; mean NW = 56.33, S.D. $=30.97$; mean $\mathrm{SW}=31.19$, S.D. $=30.50)$. Data illustrated in Figure 4, and the data analyses presented above suggest that, in the probe trial, CTR subjects searched the target quadrant (NE) more intensively than they did the other quadrants, whereas PDA patients distributed their search of the arena more evenly.

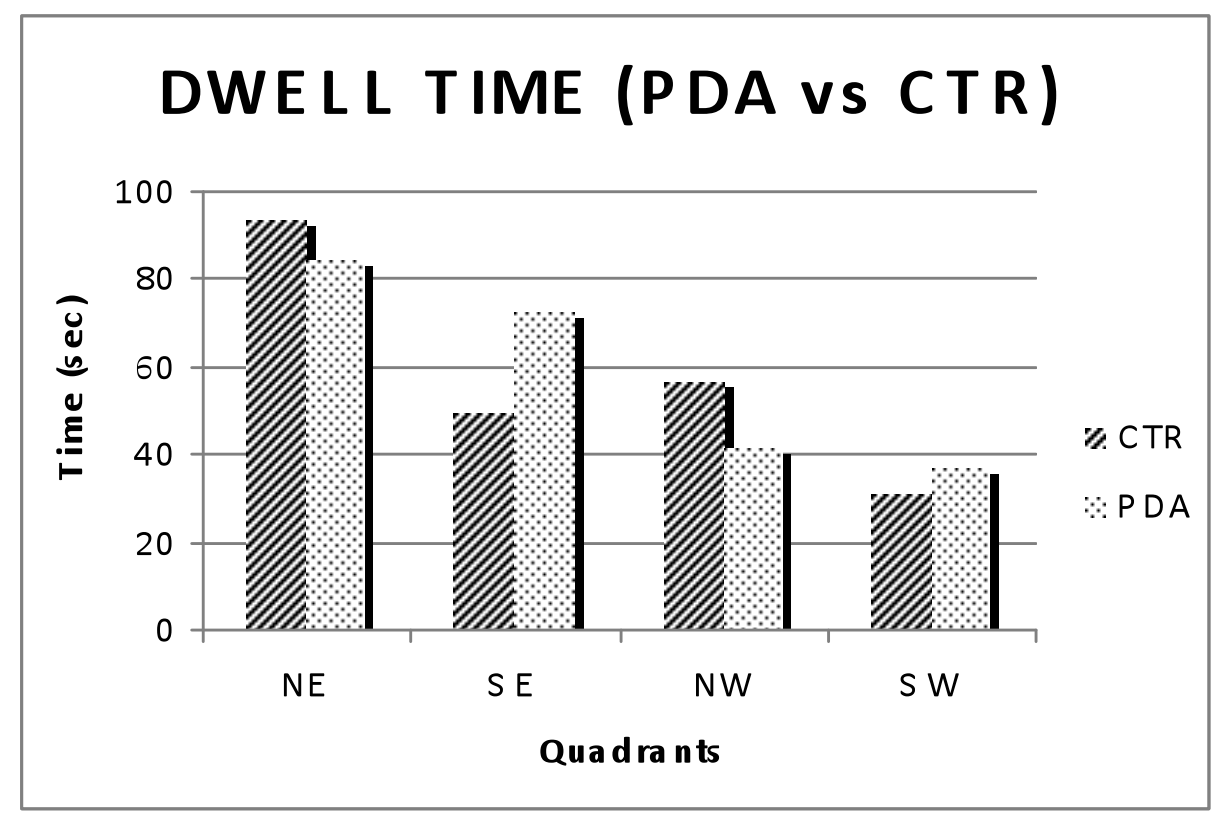

Fig. 4. The time in seconds (means and standard deviations of the mean) the 2 groups of participants searched each quadrant of the arena during the probe trial. The NE is the quadrant that contained the target from trial 1 to trial 9.

Time spent on the target

Once participants found the invisible target, they could stay on it all the time they needed in order to look around and learn its position in relation to the distal cues. An independent sample t-test revealed a significant difference $(\mathrm{t}(60)=-2.47, \mathrm{p}<0.05)$ between the mean time spent on the target by CTR $($ mean $=84.63$, S.D. $=44.14)$ and PDA $($ mean $=117.40$, S.D. $=59.14)$. 
Sex differences

A multifactorial (trials $\mathrm{x}$ groups $\mathrm{x}$ sex) repeated measures ANOVA performed on data from the acquisition trials with latency and path length as dependent variables found a significant main effect of group (latency: $F(1$, $58)=4.05$; path length: $F(1,58)=5.83)$, while the main effect of sex was not significant (latency: $\mathrm{F}(1,58)=0.62$; path length: $\mathrm{F}(1,58)=0.35)$.

Searching strategies

An examination of the individual acquisition data revealed that the means illustrated in Figures 2 and 3, and the data analyses presented above, fairly represent individual performance. In fact, observing the path maps that visually represent the single subjects' spatial navigation strategies between the 9 acquisition trials (figures 5-7), we noticed that PDA patients could be divided in two groups: some of them performed the task as the HC did, while the others had great difficulties in finding an efficient strategy to locate the target. In particular, once almost all the CTR subjects and some PDA patients located the target, they returned to it rapidly and efficiently on each subsequent trial (i.e., they showed efficient place performance); in contrast, the other PDA patients who located the target on one trial tended not to learn its position for the subsequent trials. 


\section{Chapter IV}

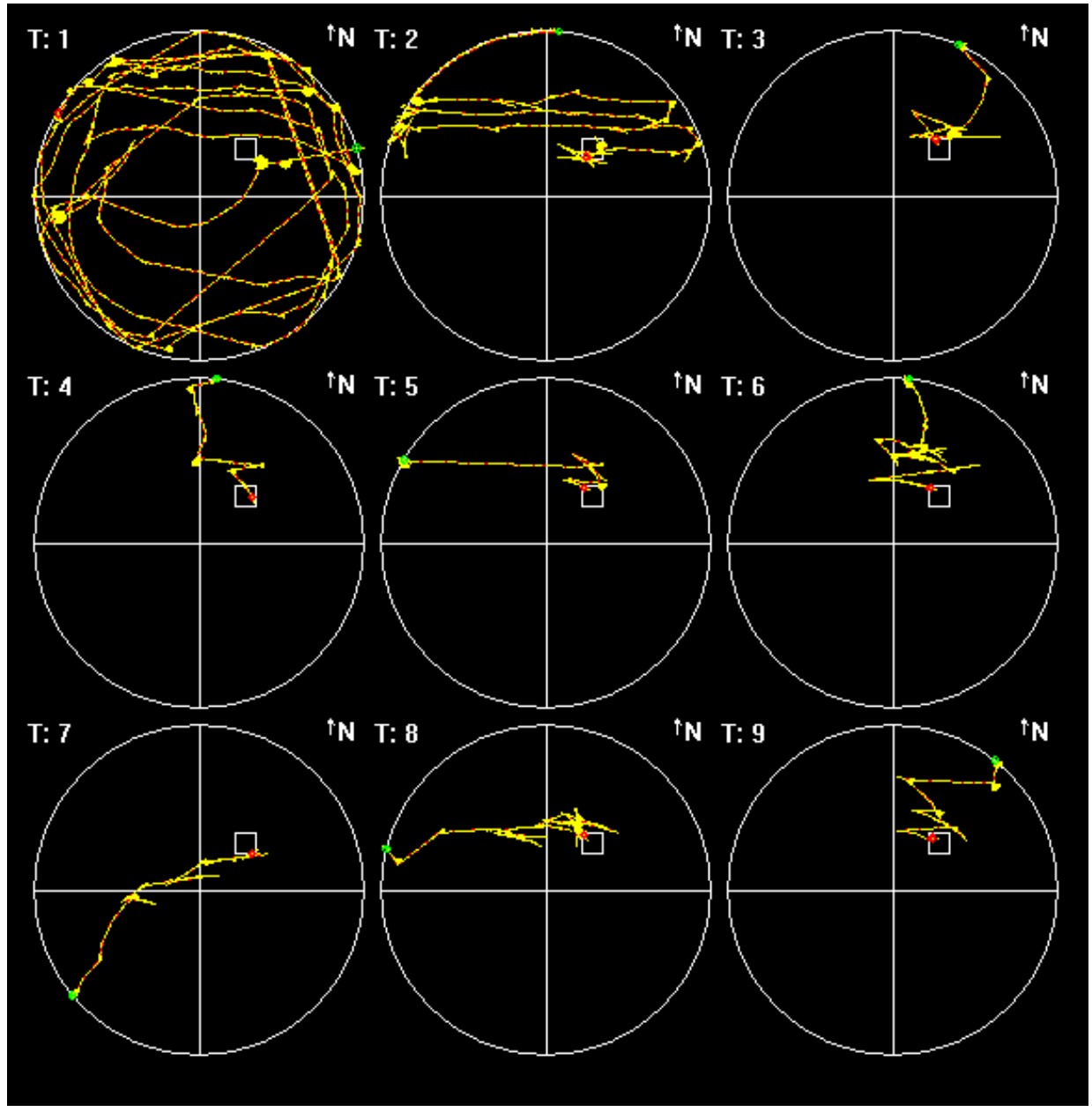

Fig. 5. Representative search paths on acquisition trials for one of the CTR subjects. Each of the trials started from a new location, while the position of the target was always the same. 
Place learning in panic disorder

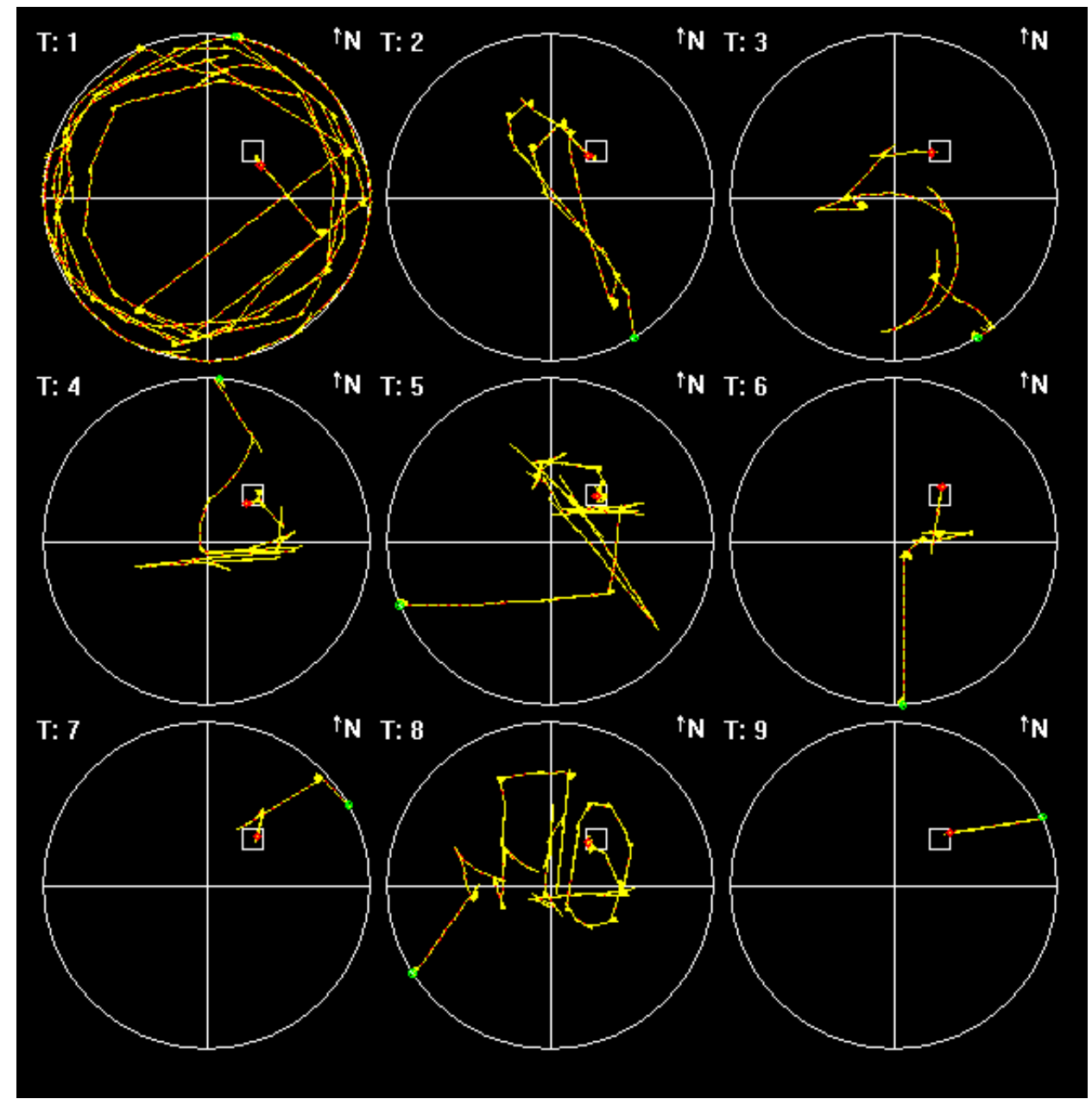

Fig. 6. Representative search paths on acquisition trials for one of the PDA patients classified as a good performer (PDA-A). Each of the trials started from a new location, while the position of the target was always the same. 


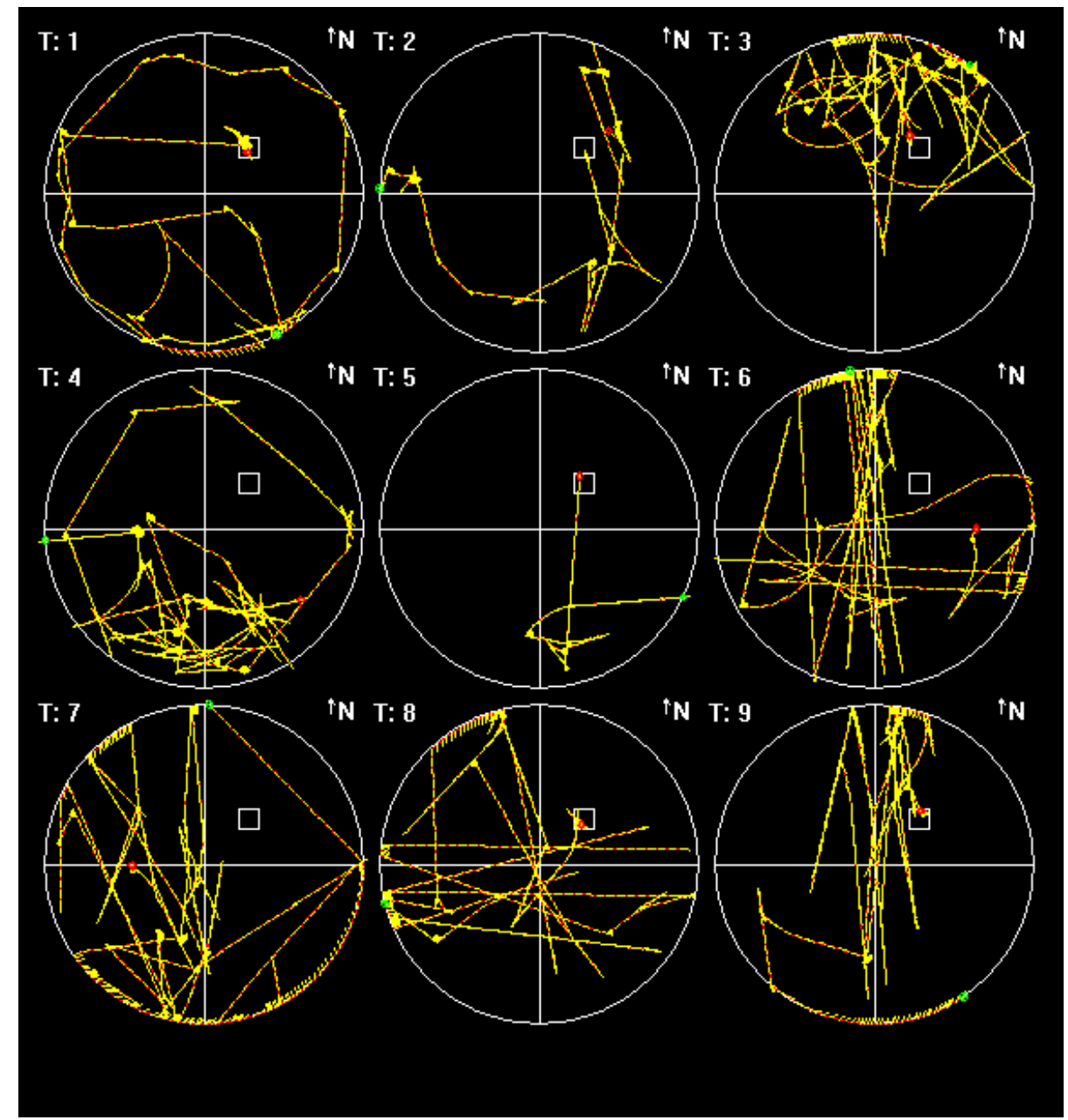

Fig. 7. Representative search paths on acquisition trials for one of the PDA patients classified as a bad performer (PDA-B). Each of the trials started from a new location, while the position of the target was always the same.

The cluster analysis performed on the PDA group for the two dependent variables (path length and latency) confirmed our impressions and allowed us to identify two subgroups of patients (PDA-A - or good performers: 17 subjects and PDA-B - or bad performers: 14 subjects). A following discriminant analysis was performed with path length and latency during the nine trials as predictor variables. Univariate ANOVAs revealed that the PDA-A and PDA-B differed significantly on latency and path length. The value of this function was significantly different for PDA-A and PDA-B patients (latency: chi-square $=64.538, \mathrm{df}=9, \mathrm{p}<0.05$; path length: chi- 
square $=29.768, \mathrm{df}=9, \mathrm{p}<0.05)$. Overall, the discriminant function successfully predicted outcome for $100.00 \%$ of cases regarding the latency and for 93.5 of cases regarding the path length.

Epidemiological and clinical variables in $P D A-A$ vs $P D A-B$ patients

No significant differences were found in education (PDA-A: mean $=11.00$ years; S.D. $=4.24$; PDA-B: mean $=11.79$; S.D. $=4.30, \mathrm{t}=-0.51(29), \mathrm{p}=$ $0.61)$, in the VAS-A (PDA-A: mean $=15.41$; S.D. $=8.10$; PDA-B: mean $=$ 16.00; S.D. $=6.38, \mathrm{t}=-0.22(29), \mathrm{p}=0.83)$, in the P\&A (PDA-A: mean $=$ 28.24; S.D. $=10.48$; PDA-B: mean $=22.77$; S.D. $=12.07, \mathrm{t}=1.32(28), \mathrm{p}=$ $0.20)$ and in the ACQ (PDA-A: mean $=25.88$; S.D. $=11.67$; PDA-B: mean $=16.14$; S.D. $=13.91, \mathrm{t}=-2.12(29), \mathrm{p}=0.06)$.

On the contrary, PDA-A and PDA-B differed in age (PDA-A: mean years $=$ 30.41; S.D. $=10.65$; PDA-B: mean $=41.71$; S.D. $=16.03, \mathrm{t}=-2.35(29), \mathrm{p}$ $<0.05)$ and in the duration of illness (PDA-A: mean = 5.18; S.D. $=5.54$; PDA-B: mean $=13.41 ;$ S.D. $=9.11, \mathrm{t}=-3.00(29), \mathrm{p}<0.05)$.

\section{Place learning}

During the 8 analyzed trials, CTR subjects and PDA-A patients tended to relocate the target more frequently than PDA-B patients did. On average, the group of CTR subjects located the target 7.6 times, and the group of PDA-A patients located it 7.7 times. In contrast, PDA-B patients, on average, located the target 5.5 times. The difference between these means is statistically significant $(\mathrm{F}(2,61)=28.39)$. Post-hoc comparisons using Fisher's LSD test confirmed that PDA-B patients located the target significantly fewer times than PDA-A and CTR subjects did.

\section{Latency}

Figure 8 illustrates the mean latency to the invisible target for each group over the series of acquisition trials. A split-plot ANOVA conducted on these data detected a significant main effect of group $(F(2,59)=28.04)$, a significant trials $\mathrm{x}$ group interaction effect $(\mathrm{F}(14,59)=2.52)$, and no significant main effect of trials. Post-hoc comparisons using Fisher's LSD test detected significant differences between the average latency of CTR subjects and PDA-B patients, and between the average latency of PDA-A and PDA-B patients, but not between CTR and PDA-A. 


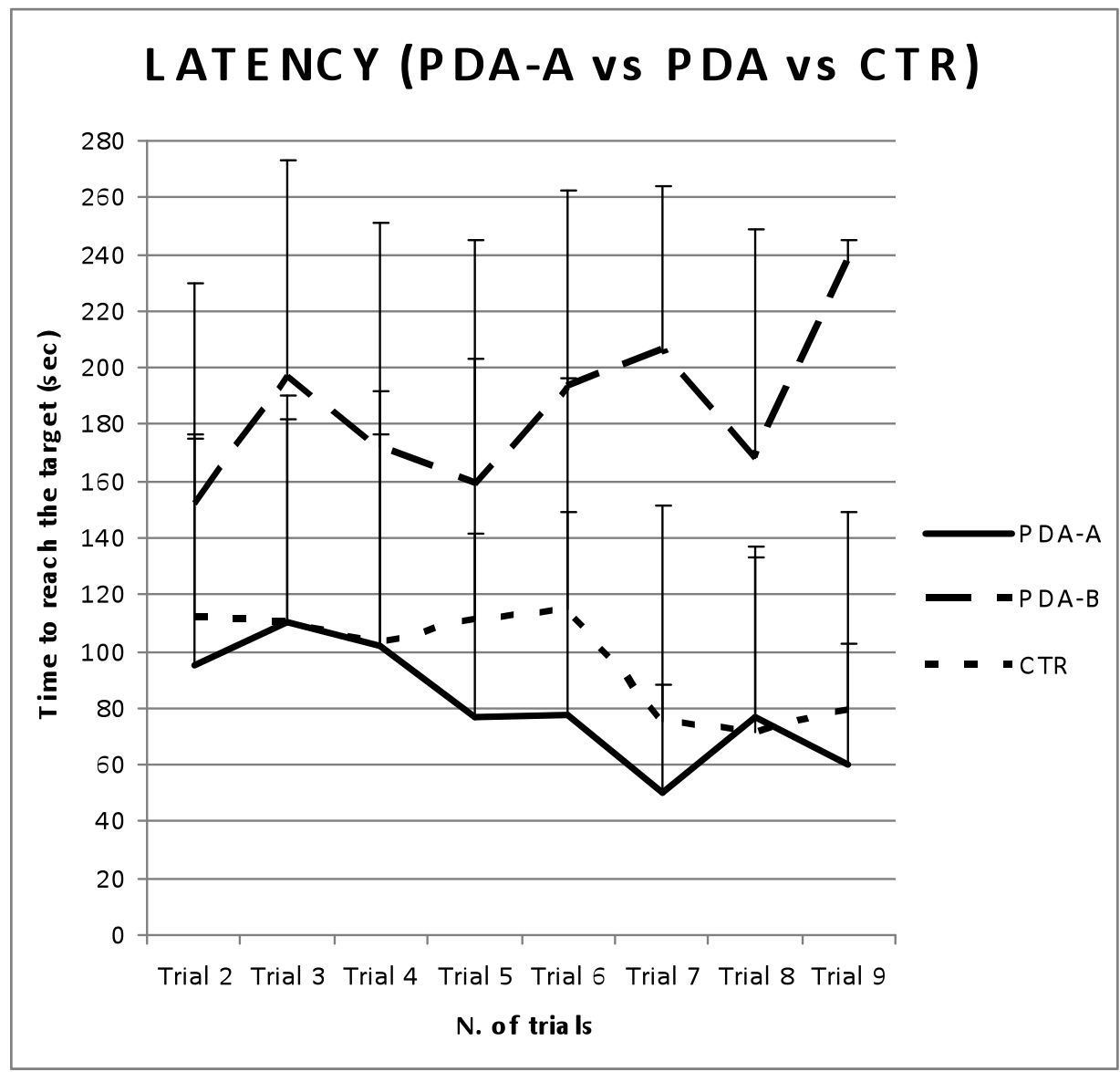

Fig. 8. The figure illustrates the time in seconds (means and standard deviations of the mean) required by the CTR and the two subgroups of PDA patients to reach the invisible target on each of the 8 acquisition trials.

\section{Path length}

Figure 9 illustrates the mean path length to the invisible target for each group over the series of acquisition trials. A split-plot ANOVA conducted on these data detected a significant main effect of group, $(\mathrm{F}(2,59)=16.87)$, a significant trials $\mathrm{x}$ group interaction effect, $(\mathrm{F}(2,59)=11.30)$, and no significant main effect of trials. Post-hoc comparisons using Fisher's LSD test detected significant differences between the average path lengths of CTR subjects and PDA-B patients, and between the average latency of PDA-A and PDA-B patients, but not between CTR and PDA-A. 


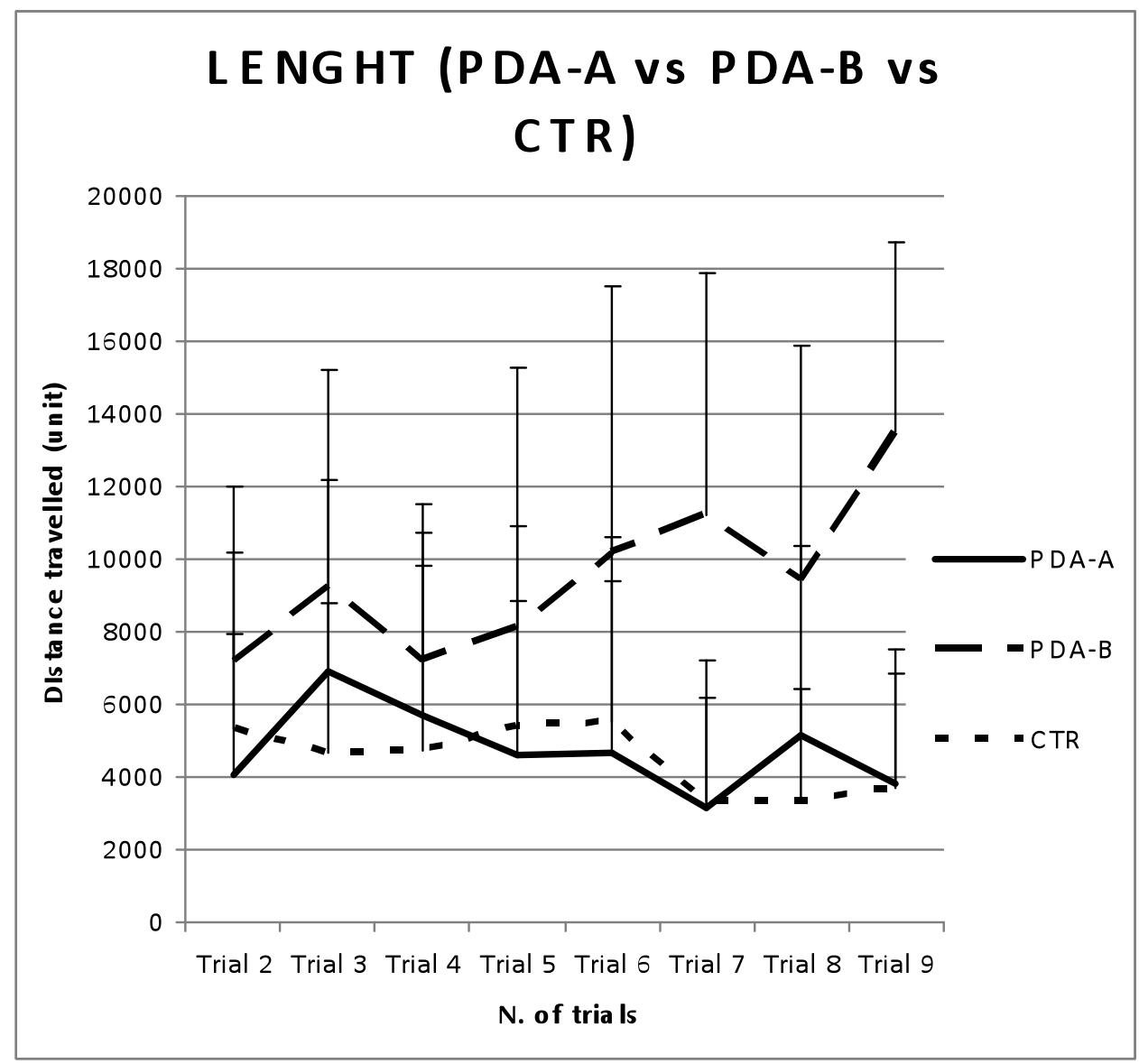

Fig. 9. The path length in units (means and standard deviations of the mean) required by the CTR and the two subgroups of PDA patients to reach the invisible target on each of the 8 acquisition trials.

Dwell time (probe trial)

Figure 10 illustrates the mean time participants in each group spent searching each quadrant during the probe trial. A split-plot ANOVA detected a significant main effect of quadrant $(\mathrm{F}(3,59)=16.60)$, a significant quadrant $\mathrm{x}$ group interaction effect $(\mathrm{F}(6,59)=2.68)$, and no significant main effect of group. Post-hoc comparisons using Fisher's LSD test detected a significant difference between the mean time CTR subjects and PDA-B patients spent in the NE quadrant (the quadrant in which the invisible target was located) and between the mean time PDA-A and PDA-B patients spent in the NE quadrant. Orthogonal post-hoc contrasts conducted on the data obtained from the CTR and the PDA-A participants revealed: (a) 
a significant difference in mean dwell time between the NE and SE quadrants, (b) a significant difference in mean dwell time between the NE and NW quadrants, and (c) a significant difference in mean dwell time between the NE and SW (CTR: mean NE = 93.57, S.D. $=50.94$; mean SE $=$ 49.18, S.D. $=35.40$; mean $\mathrm{NW}=56.33$, S.D. $=30.97$; mean $\mathrm{SW}=31.19$, S.D. $=30.52$. PDA-A: mean $\mathrm{NE}=94.69$, S.D. $=42.16$; mean $\mathrm{SE}=56.99$, S.D. $=31.03$; mean NW $=46.05$, S.D. $=26.30$; mean $\mathrm{SW}=32.81$, S.D. $=$ 23.08). On the contrary, orthogonal post-hoc contrasts conducted on the data obtained from the PDA-B subjects revealed: (a) no significant difference in mean dwell time between the NE and SE quadrants, (b) a significant difference in mean dwell time between the NE and NW quadrants, and (c) a significant difference in mean dwell time between the NE and SW (mean $\mathrm{NE}=71.94$, S.D. $=41.91$; mean $\mathrm{SE}=90.91$, S.D. $=38.22$; mean $\mathrm{NW}=$ 35.39 , S.D. $=31.68$; mean $\mathrm{SW}=41.74$, S.D. $=43.56)$. The data illustrated in Figure 10, and the data analyses presented above suggest that, on the probe trial, CTR and PDA-A subjects searched the target quadrant (NE) more intensively than they did the other quadrants, whereas PDA-B patients distributed their search of the arena more uniformly.

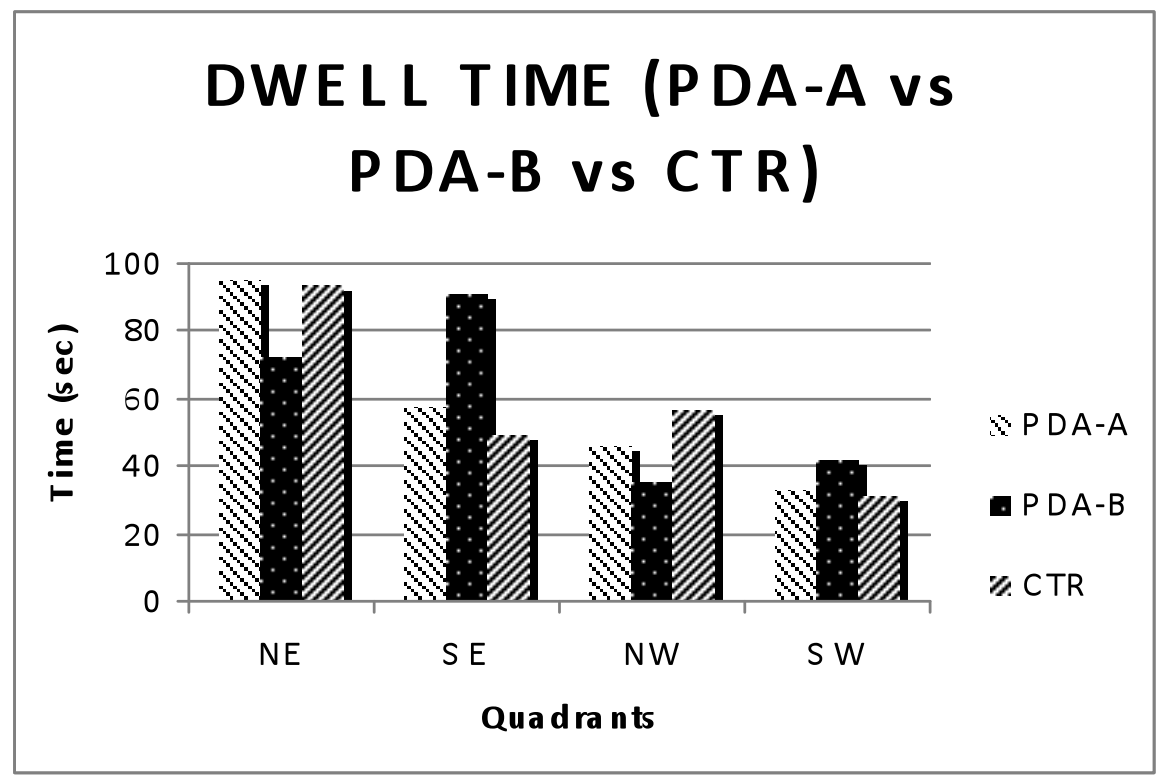

Fig. 10. The time in seconds (means and standard deviations of the mean) the CTR and the two subgroups of PDA patients searched each quadrant of the arena during the probe trial. The NE is the quadrant that contained the target from trial 1 to trial 9. 
Time spent on the target

A one-way ANOVA showed that the mean time spent on the target was significantly different in the three groups of subjects $(F(2,61)=30.48)$. In particular, post-hoc test indicated that PDA-B significantly differed from PDA-A and CTR, while PDA-A and CTR did not differed one from the other.

\section{Discussion}

The aim of this study was to investigate the place learning abilities in a sample of PDA patients compared to healthy volunteers using a virtual space. At the moment of the test, PDA patients did not present any kind of panic-related symptoms, as assessed by a brief clinical interview and the VAS-A scores. Comparing the two groups of subjects we found significant differences in latency and path length to find the target, as well as in the time spent in the NE quadrant during the probe trial. As shown by previous studies (Jacobs et al., 1997; Jacobs et al., 1998), the time and the distance required by healthy individuals to find the invisible target hidden on the $\mathrm{C}$ $\mathrm{G}$ arena floor significantly decreased across acquisition trials. Apparently this did not happen in the PDA group, in which time and distance to find the invisible target remained almost the same over the trials. Assuming a direct correspondence between the time required to find the target and the knowledge of its location, decreasing latencies to find the invisible target indicate that healthy subjects, but not patients, formulated efficient strategies to locate it over trials. This assumption was confirmed analyzing the time spent searching the hidden target when it was removed from the floor: time spent in the appropriate quadrant was greater than time spent in other quadrants in the CTR group, but not in the PDA group.

Nevertheless, an accurate examination of the individual acquisition data revealed that the means illustrated in Figures 2 and 3, fairly represent individual performance. Observing the path maps representing the single subjects' spatial navigation strategies, we unexpectedly noticed that, while there were no relevant differences within the CTR group, some of the PDA patients were "good performers", and some others were "bad performers". The statistical analysis confirmed our impressions and allowed us to divide the patients in two separate subgroups. Patients in the PDA-A group showed place learning abilities comparable to those found in the CTR subjects, while patients in the PDA-B group were unable to learn the correct 
strategies to find the invisible target. We can conclude that place learning occurred in the virtual arena only for healthy subjects and for a subgroup of patients affected by panic disorder with agoraphobia.

These data suggest that CTR subjects and some of the examined patients used distal cues and the relations among them to find the target quickly and efficiently and to remember its location, while the other patients did not. This resulted in a lack of efficient searching strategies in the patients group, who did not learn the location of the invisible target and thus performed the task using a trial-and-error approach. The fact that CTR and PDA-A subjects were faster than the others in developing the strategies necessary to find the target is also evident observing the time spent on the target once they found it: CTR and PDA-A subjects spent less time than PDA-B analyzing the environment and remembering the target position in relationship with the surrounding distal cues. Once acquired, these spatial relations are used to form a cognitive map of the external environment, and to specify locations within that map (O'Keefe and Nadel, 1978). Moreover, the use of such a map permits behavioural flexibility: when teleported to the arena, good performers became able to establish their initial location relative to the invisible target, and to use this knowledge to move directly toward its location. Apparently, these abilities were impaired in the PDA-B patients that seemed unable to create the maps useful to rapidly find the target.

The analyses of the epidemiological and clinical variables revealed interesting differences between PDA-A and PDA-B patients: the first ones were significantly younger and affected by panic disorder from less time than the latter. These differences can represent an explanation of our results. First, as specified by O'Keefe and Nadel (O'Keefe and Nadel, 1978), as well as by Thomas et al. (Thomas et al., 1999), the human cognitive mapping system changes over the lifespan, as happens in rats (Gallagher and Rapp, 1997). Second, the fact that the duration of illness is related to the subjects' performance is coherent with previous studies showing that panic disorder is associated to dysfunctional exploratory patterns (Jacobs and Nadel, 1985; Kallai et al., 2007a; Kallai et al., 1995; Kallai et al., 1999). Thus, a possible interpretation of our results is that the higher the age of patients and the longer the duration of the illness, the more likely patients develop dysfunctional cognitive and behavioral strategies that worsens their performance.

Regarding the sex differences issue, we did not find any effect of gender in our sample. This is quite surprising considering some previous studies that claim strong sex differences in navigation tasks (Astur et al., 1998; Astur et 
al., 2004), but it is consistent with Thomas et al. (Thomas et al., 1999), who failed to find an effect of gender in the $\mathrm{C}-\mathrm{G}$ arena in some 1800 participants. In conclusion, we argue that the existence of two different subgroups of patients with panic disorder and agoraphobia who differ in their spatial orientation abilities could open new perspectives in the cognitivebehavioural treatment of this diffuse and disabling disorder. As shown by Thomas and colleagues (Thomas et al., 1999), with sufficient training, bad performers (older adults in their study) can perform comparably to good performers (younger adults) on "cue learning" tasks (i.e., tasks on which they are required to navigate to a visible target). Similarly, Kallai and coll. (Kallai et al., 1999), assuming that all the PDA patients suffer of spatial problems, proposed to integrate the traditional behavioral therapy with a specific training that helps them to efficiently direct their attention to the external environment. Our data show that the C-G arena can be used as a screening tool to discriminate PDA patients with and without spatial orientation disorders, and to select those patients who need a spatial rehabilitation training. Moreover, beside the already mentioned therapeutic interest, our findings have a potential interest for a better theoretical insight into the mechanisms of panic and agoraphobia.

Finally, differently from exploration of real, poorly controlled environments, the use of a virtual space such as the C-G arena allows to deeply assess spatial abilities in PDA patients without inducing significant panic related symptoms during the test session. This represents a remarkable advantage for those who are interested in investigating patients' spatial abilities without significantly alter their neurophysiological conditions and avoiding the risk to provoke a panic attack during the assessment.

\section{Acknowledgments}

The authors would like to thank the team who developed the C-G Arena making it available for free to all researches and clinicians who want to investigate the place learning abilities in humans. 


\section{References}

1. Astur, R.S., Ortiz, M.L., Sutherland, R.J., 1998. A characterization of performance by men and women in a virtual Morris water task: a large and reliable sex difference. Behavioral Brain Research 93, 185190.

2. Astur, R.S., Tropp, J., Sava, S., Constable, R.T., Markus, E.J., 2004. Sex differences and correlations in a virtual Morris water task, a virtual radial arm maze, and mental rotation. Behavioural Brain Research 151, 103-115.

3. Bandelow, B., 1995. Assessing the efficacy of treatments for panic disorder and agoraphobia. II: The panic and agoraphobia scale. International Clinical Psychopharmacology 10, 73-81.

4. Boldrini, M., Del Pace, L., Placidi, G.P.A., Keilp, J., Ellis, S.P., Signori, S., Placidi, G.F., Cappa, S.F., 2005. Selective cognitive deficits in obsessive-compulsive disorder compared to panic disorder with agoraphobia. Acta Psychiatrica Scandinavica 111, 150-158.

5. Chambless, D.L., Caputo, G.C., Bright, P., Gallagher, R., 1984. Assessment of fear in agoraphobics: The Body Sensations Questionnaire and the Agoraphobic Cognitions Questionnaire. Journal of Consulting and Clinical Psychology 52, 1090-1097.

6. Dupont, H., Mollard, E., Cottraux, J., 2000. Visuo-spatial attention processes in panic disorder with agoraphobia: a pilot study using a visual target discrimination task. European Psychiatry 15, 254-260.

7. Gallagher, M., Rapp, P.R., 1997. The use of animal models to study the effects of aging on cognition. Annual Review of Psychology 48, 339-370.

8. Gillner, S., Mallot, H.A., 1998. Navigation and acquisition of spatial knowledge in a virtual maze. Journal of Cognitive Neuroscience 10, 445-463.

9. Gladsjo, J.A., Rapaport , M.H., McKinney, R., Lucas, J.A., Rabin, D.A., Oliver, T., Davis, J., Auerbach, M., Judd, L.L., 1998. A neuropsychological study of panic disorder: Negative findings. Journal of Affective Disorders 49, 123-131.

10. Iavarone, A., Del Castello, E., Ruggiero, G., Iachini, T., 2005. Do panic-agoraphobics overestimate distances? . The World Journal of Biological Psychiatry 6, 242-246. 
11. Jacobs, W.J., Laurance, H.E., Thomas, K.G., 1997. Place Learning in Virtual Space I: Acquisition, Overshadowing, and Transfer. Learning and Motivation 28, 521-541.

12. Jacobs, W.J., Nadel, L., 1985. Stress-induced recovery of fear and phobias. Psychological Review 92, 512-531.

13. Jacobs, W.J., Thomas, K.G., Laurance, H.E., Nadel, L., 1998. Place Learning in Virtual Space II. Topographical Relations as One Dimension of Stimulus Control. Learning and Motivation 29, 288308.

14. Kallai, J., Karadi, K., Bereczkei, T., Rozsa, S., Jacobs, W.J., Nadel, L., 2007a. Spatial exploration behaviour in an extended labyrinth in patients with panic disorder and agoraphobia. Psychiatry Research 149, 223-230.

15. Kallai, J., Koczan, G., Szabo, I., Molnar, P., Varga, J., 1995. An experimental study to operationally define and measure spatial orientation in panic agoraphobic subjects, generalised anxiety and healthy control groups. Behavioural and Cognitive Psychotherapy 23, 145-152.

16. Kallai, J., Kosztolanyi, P., Osvath, A., Jacobs, W.J., 1999. Attention fixation training: Training people to form cognitive maps help to control symptoms of panic disorder with agoraphobia. Journal of Behavioural Therapy and Experimental Psychiatry 30, 273-288.

17. Kallai, J., Makany, T., Csatho, A., Karadi, K., Horvath, D., KovacsLabadi, B., Jarai, R., Nadel, L., Jacobs, J.W., 2007b. Cognitive and affective aspects of thigmotaxis strategy in humans. Behavioural Neuroscience 121, 21-30.

18. Kallai, J., Makany, T., Karadi, K., Jacobs, W.J., 2005. Spatial orientation strategies in Morris-type virtual water task for humans. Behavioral Brain Research 159, 187-196.

19. Kallai, J., Szabados, Z., Varga, J., Ozsvath, K., Molnar, P., Koczan, G., 1996. Opposite asymmetries in blind locomotor orientation of patients with panic agoraphobia compared to those with generalized anxiety. International Journal of Psychophysiology 23, 155-161.

20 Keogh, E., French, C.C., 1999. The effect of trait anxiety and mood manipulation on the breadth of attention. European Journal of Personality 13, 209-223.

21. May, M., Pèruch, P., Savoyant, A., 1995. Navigating in a virtual environment with map-acquired knowledge: Encoding and alignment effects. Ecological Psychology 7, 21-36. 
23. Morris, R.G.M., 1981. Spatial localization does not require the presence of local cues. Learning and Motivation 12, 239-260.

24. Nadel, L., Thomas, K.G.F., Laurance, H.E., Skelton, R., Tal, T., Jacobs, W.J. 1998. Human place learning in a computer generated arena. In: C. Freksa, C. Habel, \& K. F. Wender (Eds.), An interdisciplinary approach to representing and processing spatial knowledge, Springer, Berlin, pp. 399-427.

25. O'Keefe, J., Nadel, L. 1978. The Hippocampus as a cognitive map. Clarendon Press. Oxford, UK.

26. Parsons, T.D., Silva, T.M., Pair, J., Rizzo, A.A., 2008. Virtual environment for assessment of neurocognitive functioning: virtual reality cognitive performance assessment test. Studies in Health Technology and Information 135, 351-356.

27. Péruch, P., Vercher, J., Gauthier, G.M., 1995. Acquisition of spatial knowledge through visual exploration of simulated environments. Ecological Psychology 7, 1-20.

28. Purcell, R., Maruff, P., Kyrios, M., Pantelis, C., 1998. Neuropsychological deficits in obsessive-compulsive disorder: a comparison with unipolar depression, panic disorder, and normal controls. Archives of General Psychiatry 55, 415-442.

29. Rizzo, A.A., Buckwalter, J.G., Neumann, U., Kesselman, C., Thiebaux, M., 1998. Basic issues in the application of virtual reality for the assessment and rehabilitation of cognitive impairments and functional disabilities. Cyberpsychology and Behavior 1, 59-78.

30. Rose, F.D., Attree, E.A., Johnson, D.A., 1996. Virtual reality: An assistive technology in neurological rehabilitation. Current Opinion in Neurology 9, 461-467.

31. Ruddle, R.A., Payne, S.J., Jones, D.M., 1997. Navigating buildings in "desk-top" virtual environments: Experimental investigations using extended navigational experience. Journal of Experimental Psychology: Applied 3, 143-159.

32. Sheehan, D.V., Lecrubier, Y., Sheehan, K.H., Amorim, P., Janavs, J., Weiler, E., 1998. The Mini-International Neuropsychiatric Interview (M.I.N.I.): the development and validation of a structured diagnostic psychiatric interview for DSM-IV and ICD-10. Journal of Clinical Psychiatry 59 (Suppl), 22-33.

33. Taylor, C.B., Sheikh, J., Agras, W.S., Roth, W.T., Margraft, J., Ehlers, A., Maddock, R.J., Grossard, D., 1986. Ambulatory heart rate changes in patients with panic attacks. American Journal of Psychiatry 143, 478-482. 
34. Thomas, K.G., Hsu, M., Laurance, H.E., Nadel, L., Jacobs, W.J., 2001. Place learning in virtual space. III: Investigation of spatial navigation training procedures and their application to fMRI and clinical neuropsychology. Behavior Research Methods, Instruments \& Computers 33, 21-37.

35. Thomas, K.G., Laurance, H.E., Luczak, S.E., Jacobs, W.J., 1999. 36. Age-related changes in a human cognitive mapping system: data from a computer-generated environment. Cyberpsychology and Behavior 2, 545-566.

36. Viaud-Delmon, I., Berthoz, A., Jouvent, R., 2002. Multisensory integration for spatial orientation in trait anxiety subjects: absence of visual dependence. European Psychiatry 17, 194-199.

37. Viaud-Delmon, I., Siegler, I., Israel, I., Jouvent, R., Berthoz, A., 2000. Eye deviation during rotation in darkness in trait anxiety: an early expression of perceptual avoidance? Biological Psychiatry 47, $112-118$

38. Waller, D., 2000. Individual differences in spatial learning from computer simulated environments. Journal of Experimental Psychology: Applied 6, 307-321.

39. Wang, R.F., 1999. Representing a stable environment by egocentric updating and invariant representations. Spatial Cognition and Computation 1, 431-445.

40. Wilson, P., Foreman, N., Tlauka, M., 1997. Transfer of spatial information from a virtual to a real environment. Human Factors 39, 526-531.

41. Wittchen, H.U., Hoyer, J., 2001. Generalized anxiety disorder: Nature and course. Journal of Clinical Psychiatry 62, 15-19. 



\section{INTERMEZZO}

\section{Virtual worlds, real healing}

A. Gorini, A. Gaggioli, G. Riva.

Science. 2007, Dec 21; 318(5856):1549. 



\section{LETTERS}

edited by Jennifer Sills

\section{Virtual Worlds, Real Healing}

IN THE SOCIAL COGNITION SPECIAL SECTION, G. MILLER EXPLAINED how virtual worlds such as Second Life have become a fertile ground for psychologists exploring human behavior ("The promise of parallel universes," 7 September, p. 1341). In addition to the important social applications mentioned in Miller's article, online communities are playing an emerging role in health services.

Compared with the traditional telehealth systems (i.e., chat, e-mail, and videoconference), online virtual worlds provide the remote user, or patient, with a feeling of embodiment that has the potential to facilitate the clinical communication process and to positively influence group cohesiveness in group-based therapies. It may also create higher levels of interpersonal trust (1), which is a fundamental requirement for establishing a successful therapeutic alliance.

Recent evidence has shown that virtual reality-based treatments effectively combat anxiety disorders (2) and allow subjects to develop real-world skills starting from virtual experiences (3). These successes raise the possibility of creating online immersive therapeutic environments for specific disorders. Imagine, for example, a patient with a social phobia who avoids any interaction with other people. After a number of face-to-face sessions with a therapist, the patient can use his personal avatar to explore a virtual environment, such as a virtual pub in which he can ask the barman for a drink. In the following sessions, other people progressively enter the same virtual pub (they can be other patients, for example) and interact with the patient until he can develop efficient social contacts. The therapist can remotely monitor

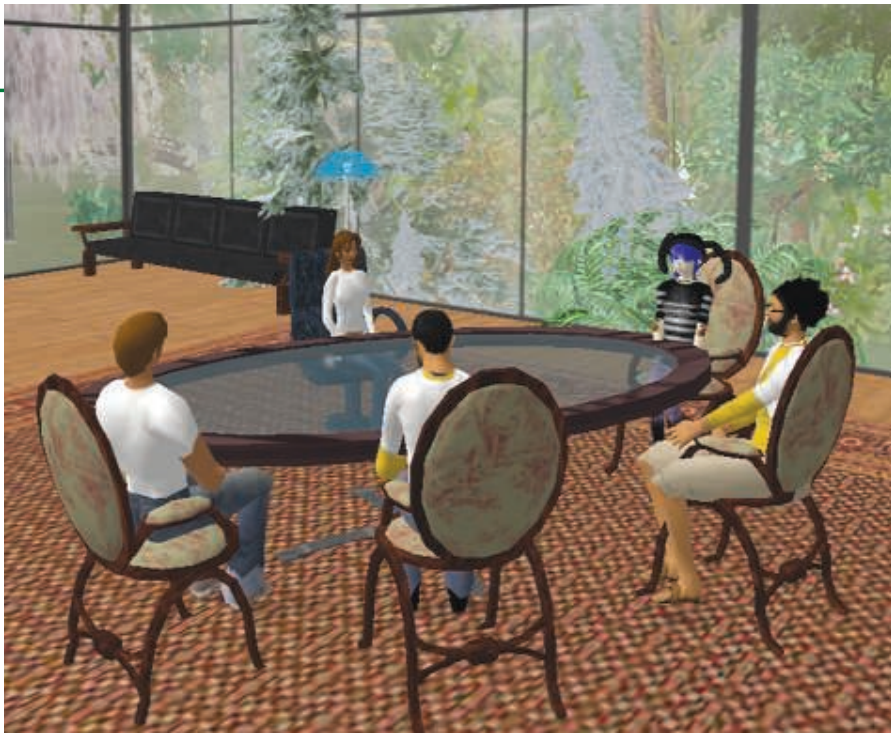

Virtual therapy. An example of a group support therapy scenario in Second Life.

the patient's psychological, physiological, and emotional responses with the use of biomonitoring systems and can modify the intervention on the basis of the therapeutic needs. This is just one example of the promise of virtual worlds in clinical settings.

\section{ALESSANDRA GORINI}

Applied Technology for Neuro-Psychology Laboratory, Istituto Auxologico Italiano, 20146 Milan, Italy.

\section{References}

1. G. Bente, S. Rüggenberg, N. C. Krämer, paper presented at the 8th International Workshop on Presence, London, 21 to 23 September 2005.

2. L. Gregg, N. Tarrier, Soc. Psychiatry Psychiatr. Epidemiol. 42,343 (2007)

3. G. Riva, CyberPsychol. Behav. 8, 220 (2005).

\section{The Age-Old Question of Researcher Innovation}

UNFORTUNATELY, BOTH Y. BHATTACHARJEE ("The young and the innovative," ScienceScope, 21 September, p. 1663) and Jeremy Berg, director of the National Institute of General Medical Sciences, perpetuate the myth that "[e]arly-career types are historically the ones who come up with the most innovative ideas." Though this myth remains popular, the available empirical evidence suggests that middle-aged scientists are (i) more apt than young scientists to make revolutionary discoveries $(1,2)$ and (ii) more productive than young scientists (3). In fact, young scientists are not even especially prone to accept a new theory before older scientists $(4,5)$. It is distressing that funding agencies are making important decisions on the basis of a popular myth that has been examined empirically.

\section{K. BRAD WRAY}

Department of Philosophy, State University of New York, Oswego, NY 13126, USA.

\section{References}

1. K. B. Wray, Soc. Stud. Sci. 33, 1 (2003).

2. H. Zuckerman, Scientific Elite: Nobel Laureates in the United States (Transaction Publishers, New Brunswick, N], 1996).

3. S. Cole, Am. J. Soc. 84, 4 (1979).

4. D. L. Hull, P. D. Tessner, A. M. Diamond, Science 202, 717 (1978).

5. P. Messeri, Soc. Stud. Sci. 18, 1 (1988).

\section{Response}

IN HIS LETTER, WRAY POINTS OUT THAT EMPIRIcal data suggests that many revolutionary discoveries are made by "middle-aged scientists." He cites studies that reveal that 24 scientists discussed in Thomas Kuhn's “The Structure of Scientific Revolutions" had a mean age of 37.4 when they made their revolutionary contributions (1) and that a large collection of Nobel laureates had a mean age of 38.7 at the time of their prize-winning discoveries (2). The mean age of the NIH Director's New Innovator Awardees who have just received their first substantial independent research funding from the NIH is approximately 37 , somewhat younger than the mean age for new NIH R01 grantees of 41. One of the 



\section{CHAPTER V}

\section{New technologies and relaxation: an explorative study on obese patients with emotional eating}

G. M. Manzoni, A. Gorini, A. Preziosa, F. Pagnini, G. Castelnuovo, E. Molinari, G. Riva.

Journal of CyberTherapy and Rehabilitation. 2008, 1(2): 182-192. 



\section{Abstract}

Since stress and negative emotions are critical factors in inducing overeating in obese patients, psychological and behavioral interventions for obesity should include stress management techniques. A three weeks relaxation protocol supported by the use of new technologies, including virtual reality (VR) and portable mp3 players, was developed in order to reduce stress and related emotional eating episodes in obese patients. Sixty female obese inpatients reporting emotional eating were included in the study and divided in three experimental groups (virtual reality-VR, imaginative-IM and waiting list). Psychometric and physiological variables were collected. Results show that relaxation training was effective in improving perceived self efficacy in eating control, as well as in decreasing depressive symptoms, anxiety and physiological arousal both in the VR and IM conditions.

This study suggests that relaxation training for obese patients with emotional eating is effective, even if the lack of differences between the two conditions suggests some important critical considerations. 


\section{Introduction}

The interest in eating-related issues reflects worldwide statistics showing that approximately 1.6 billion adults (age 15+) are overweight and at least 400 million are obese. The World Health Organization (WHO) further projects that by 2015, approximately 2.3 billion adults will be overweight and more than 700 million will be obese (WHO, 2006). Given that overweight and obesity lead to serious health consequences, developing and implementing effective interventions for substantially reduce weight and the associated risks for health is compelling.

Beside some important and well established etiological factors such as excessive food intake and lack of physical activity, that are the principal targets in the majority of traditional weight reduction programs based on diet and exercise training, an important variable that must be taken into account for the treatment of obesity regards the way in which food intake relates with bio-psychological stress (Gluck, 2006; Volkow \& Wise, 2005). This relationship is well illustrated in the behavioral phenomenon of emotional eating, that is defined as eating in response to one's mood or emotion (Bekker, van de Meerendonk, \& Mollerus, 2004), as opposed to eating in response to physiological cues of hunger, eating on a schedule, or eating socially.

Typically, emotional eaters eat in response to negative emotions (Lindeman \& Stark, 2001), as eating in response to positive emotions has not been demonstrated to be as damaging to efforts to maintain a healthy weight. For emotional eaters, the emotion related eating behavior may be a form of inappropriate coping mechanism for alleviating and dealing with stress and negative emotions (Carver, Scheier, \& Weintraub, 1989; Popkess Vawter, Wendel, Schmoll, \& O'Connell, 1998; Solomon, 2001; Timmerman \& Acton, 2001; Troop, 1998). A recent study conducted by Ozier and coll. (Ozier et al., 2008) has found that individuals who eat in response to emotions and stress are more likely to be overweight or obese. This finding is in accord with similar data (Blair, Lewis, \& Booth, 1990) showing that patients who decreased their emotional eating lost substantially more weight than those who did not. Similarly, other two studies have demonstrated that there is a relationship between emotional eating and binge eating with higher caloric intake (Braet \& Van Strien, 1997; Waters, Hill, \& Waller, 2001). Furthermore, Geliebter and Aversa (Geliebter \& Aversa, 2003) has found that overweight individuals have substantially greater eating ratings, indicating a greater urge to eat in response to negative emotions and 
situations, than normal weight subjects. These studies support the hypothesis that overweight and obese individuals might lack appropriate strategies to cope with daily stressors and/or that their existing coping mechanisms are ineffective (Crowther, Sanftner, Bonifazi, \& Shepherd, 2001), since they lead them to use eating or overeating as a maladaptive way of coping.

If stress and negative emotions are critical factors that may induce eating, overeating and choice of palatable and thus high caloric food, then psychological and behavioral interventions for obese patients with emotional eating should also include stress management and emotionshifting strategies. Together with the traditional cognitive behavioral techniques like cognitive restructuring, self-monitoring and social support (Forety \& Carlos Poston II, 1998). Ong et al. (Ong, Linden, \& Young, 2004) found that one of the most common treatment for stress management is cognitive-behavioral therapy (CBT) associated to relaxation-oriented techniques. However, relaxation is difficult to be achieved in real life situations and the traditional relaxation methods usually take a very long time to be learned. Furthermore, given that all the main relaxation techniques resort to the use of positive mental images to facilitate the induction of the response of psycho-physical calm. The effectiveness of the interventions depends, to a large extent, on the ability of individuals to produce the relaxing images proposed by the therapist or by specific audionarratives (Vincelli, 1999). Regarding this issue, Freeman et al. (J. Freeman, Lessiter, Keogh, Bond, \& Chapman, 2004) explored whether the effects on mood and anxiety of a therapeutic narrative based on standard controlled breathing techniques were enhanced through its presentation within an audio-visual virtual environment. The results show that the presentation of the narrative within the virtual "relaxation island" in a one session experience resulted in significantly greater increase in relaxation compared to presentation of the narrative alone.

In a more recent pilot study Riva et al. (Riva, Manzoni, Villani, Gaggioli, \& Molinari, 2008), starting from these promising results, found that a brief relaxation training protocol ( 2 sessions) performed using relaxing narratives and virtual reality (VR) immersion was more effective than the same protocol provided through a DVD video in reducing anxiety in a sample of obese female inpatients with a history of emotional eating. Riva et al. (Riva, Preziosa, Grassi, \& Villani, 2006) also explored the effects on stress of mobile phones playing audio-visual relaxing narratives in a sample of Italian commuters. Once again, they showed that the use of new technologies (a combination of VR environments and mobile phones) was 
more effective in reducing stress level than commercial relaxing videos. In particular, commuters who experienced the mobile relaxing narratives virtual audio-visual experiences implemented on UMTS/3G phones - were able to obtain a significant reduction of their stress levels that the other participants did not achieve.

These results, although preliminary, suggest that new technologies, in particular VR and portable devices playing mobile narratives, may be an effective mood induction media, which can play a significant part in dealing with stress and common psychiatric disorders such as anxiety and depression (Waterworth \& Waterworth, 2004). Starting from these observations and exploratory study to observe the effect of a three-week relaxation training protocol partially provided through a relaxing VR environment and supported by portable $\mathrm{mp} 3$ players on stress and negative emotions in a sample of obese female patients with emotional eating. Furthermore, at the end of the treatment, different virtual environments representing critical situations were developed to expose the patients to the stimuli that usually produce negative emotional reactions, in order to test their ability to put in practice the acquired knowledge about relaxation. The effects of this treatment were compared to an eyes closed, imaginative, narrative-only condition and with a waiting list condition. In particular, we wanted to investigate if VR and $\mathrm{mp} 3$ players can facilitate relaxation training in a sample of obese female patients with emotional eating.

\section{Materials and Method}

\section{Participants}

Sixty female obese inpatients with emotional eating were consecutively recruited from a reference population admitted for clinical and rehabilitative residential treatment at San Giuseppe Hospital, Istituto Auxologico Italiano, Verbania, Italy, during a seven-months period. Obesity status was ascertained by a Body Mass Index (BMI) $\geq 30$ (WHO, 2006). Height was measured before treatment with a stadiometer and weight was assessed with the participant in lightweight clothing with shoes removed, on a balance beam scale.

In order to be included in the study, patients had to meet the following inclusion criteria:

- gender: female

- age: 18-60 
- primary diagnosis: obesity, in accord to the WHO criteria.

- presence of recurrent episodes of emotional eating, as assessed through the Emotional Overeating Questionnaire (EOQ) (Masheb \& Grilo, 2006).

Diagnostic interviews were conducted by an independent clinical psychologist as part of his clinical work. Patients affected by other psychiatric, psychological or neurological disorders were excluded from the study. No patients dropped-out the study.

Patients who met the inclusion criteria and gave their written informed consent to participate were randomly assigned to 3 conditions: (a) virtual reality (VR) condition; (b) imaginative condition (IM); (c) waiting list condition (WL). The randomization scheme was generated by using the Web site Randomization.com (www. randomization.com). Detailed epidemiological, clinical and demographic characteristics of the sample are summarized in table 1. No significant differences were found in all these variables between the three groups of patients. The study received ethical approval by Ethical Committee of the Istituto Auxologico Italiano.

\section{Experimental procedure and clinical protocol}

The experimental design consisted of three independent factors (three groups) and multiple repeated measures (pre-post sessions and pre-post treatment measurements).

During the initial diagnostic interview, participants were provided with detailed information about the study and the treatments. The inpatient program (IP) lasted 5 weeks. The additional treatments (VR- and IM-based relaxation training) were administered by two chartered clinical psychologists and one chartered psychotherapist under the supervision of a senior psychotherapist. The three therapists were randomized between the two conditions. 
Chapter V

\begin{tabular}{|l|l|l|l|l|l|l|}
\hline \multirow{2}{*}{ Group } & \multicolumn{2}{|c|}{$\begin{array}{c}\text { VR } \\
(\mathrm{n}=20)\end{array}$} & \multicolumn{2}{c|}{$\begin{array}{c}\text { WL } \\
(\mathrm{n}=20)\end{array}$} \\
& Mean & SD & Mean & SD & Mean & SD \\
\hline BMI & 41.74 & 3.94 & 41.82 & 4.72 & 43.21 & 6.48 \\
\hline Age & 42.80 & 11.44 & 48.55 & 7.96 & 39.65 & 14.52 \\
\hline & & & & & & \\
\hline Marital Status & Frequency & $\%$ & Frequency & $\%$ & Frequency & $\%$ \\
\hline Single & & & & & & \\
\hline Married & 8 & 40 & 4 & 20 & 10 & 50 \\
\hline Divorced & 10 & 50 & 14 & 70 & 9 & 45 \\
\hline Education & 2 & 10 & 2 & 10 & 1 & 5 \\
\hline Less than 5 years & 0 & & & & & \\
\hline Between 5 and 8 years & 7 & 35 & 4 & 20 & 6 & 30 \\
\hline Between 8 and 13 years & 9 & 45 & 11 & 55 & 8 & 40 \\
\hline More than 18 years & 4 & 20 & 3 & 15 & 4 & 20 \\
\hline Job Activity & & & & & & \\
\hline Student & 0 & 0 & 1 & 5 & 3 & 15 \\
\hline Housewife & 6 & 30 & 6 & 30 & 4 & 20 \\
\hline Employed & 11 & 55 & 10 & 50 & 11 & 55 \\
\hline Unemployed & 3 & 15 & 3 & 15 & 2 & 10 \\
\hline & & & 2 & 10 & 2 & 10 \\
\hline & & & & & \\
\hline
\end{tabular}

Tab. 1. Epidemiological, clinical and demographic characteristics of the sample

Integrated multimodal medically-managed inpatient program

All patients underwent a 5-week medically-managed, residential program consisting in a moderately low-calorie diet $(80 \%$ of the basal energy consumption estimated according to the Harris-Benedict equation), physical training, psychological support and participation in nutritional groups. The individual psychological sessions, lasting 45 minutes each, were administered once a week for five weeks and were cognitive-behavioral oriented. Contents were mainly based upon: stimulus-controlling techniques, drawing up a list of activities to dysfunctional eating behavior, problem-solving techniques aimed at coping with interpersonal situations capable of triggering emotional eating episodes, analysis and modification of dysfunctional thinking and cognitive distortions, and self-empowerment. 


\section{Relaxation training}

The experimental protocol, consisting in 4 sessions per week (12 sessions in total), lasted 3 weeks. It usually started at the beginning of the second week of the inpatient program and ended the week before the last one. The two experimental conditions (VR and IM) differed only in some sessions and the protocol was organized as following:

- Session 1 (VR and IM groups): initial assessment and brief introduction to the rationale and goals of the protocol;

- Sessions 2-4 (VR and IM groups): the patient starts to learn the relaxation techniques guided by a relaxing recorded narrative played by a computer;

- Session 5 (VR and IM groups): each patient discusses with the therapist recent episodes of emotional eating (self-monitoring) and her impressions and comments about the protocol;

- Sessions 6-8 (VR group): the patient is immersed in the Green Valley, a virtual relaxing environment, and is asked to relax herself following the relaxing narrative, after moving around the environment according to the directions suggested by the audio narrative;

- Sessions 6-8 (IM group): the patient is asked to imagine a relaxing environment representing a green valley and to relax herself listening to the audio narrative;

- Sessions 9-11 (VR group): the patient is exposed to a pre-selected virtual environment referring to a real-life situation that usually causes stress and consequently emotional eating episodes (i.e. a kitchen, a restaurant, an office, etc.). Immediately after the virtual exposure to the stressful environment, a relaxing narrative guides the patient through the relaxation process;

- Sessions 9-11 (IM group): the patient is asked to imagine a real-life situation that usually provokes stress and, consequently, emotional eating. After that, a relaxing narrative guides her through the relaxation process;

- Session 12 (VR and IM group): conclusion and final assessment.

Each session lasted about one hour; it usually started with a brief introduction and homework revision, and finished with some comments about the experience (debriefing). Starting form session 2 until the end of the treatment, participants received a portable mp3 player containing the narratives they listened during the sessions (also called mobile narratives). They were asked to use it as many times as they wanted to practice relaxation by their own without the therapist. This task was useful to speed up and boost the learning of the relaxation techniques.

In order to facilitate relaxation, during the treatment sessions patients were 
seated on a comfortable armchair in a dark room. Participants included in the IM group listened to the narrative with their eyes closed, while those in the VR group wore an head mounted display (HMD) for immersion into the virtual environment.

\section{Psychometric assessment}

The following psychometric questionnaires were administered only during the first and the last sessions:

- Beck Depression Inventory - BDI (Beck \& Steer, 1993);

- Weight Efficacy Life-Style Questionnaire - WELSQ (Clark, Abrams, Niaura, Eaton, \& Rossi, 1991).

In addition, the following psychometric questionnaires were administered before and after each treatment session:

- State Anxiety Inventory-STAI (Spielberger, Gorsuch, \& Lushene, 1970);

- Visual Analogue Scales (VAS): a variation of Gross \& Levenson's measure (Gross \& Levenson, 1995) assessing relaxation (VAS-R).

\section{Psychophysiological assessment}

Immediately before and after each treatment session, the Procomp Infiniti Biofeedback system was used to record the heart rate (HR) in order to obtain an objective measure of the internal state of the patients.

\section{The relaxing narratives}

The narratives consisted of a combination of different relaxation techniques mainly based on the Progressive Muscular Relaxation (PMR) (Borkovec \& Costello, 1993) and the Applied Relaxation (AR) (Ost \& Breitholtz, 2000). The PMR directly targets muscular tension and relaxation, while the aim of AR is to demonstrate relaxation as an effective coping strategy for significantly reducing anxiety under many daily-life circumstances. During the second week of treatment, in the VR group the narratives were presented together with the Green Valley in order to create an audio-video experience that enhanced the effect of the relaxing experience (D. Freeman, 2003; J. Freeman et al., 2004).

\section{The virtual environment software}

Two different virtual environments included in the open-source software NeuroVR 1.5 were used (Riva, Gaggioli et al., 2007). For the relaxation sessions, the Green Valley, a very relaxing environment showing a mountain landscape around a calm lake, is presented together with a 
relaxing narrative and soft sounds (birds' songs, water flowing, etc). Participants were asked to walk around the lake, to observe the nature and, after few minutes, to virtually seat on a comfortable deck chair, in order to become easily relaxed. For the final part of the protocol, patients were presented with specific virtual stressful environments simulating real-life situations that usually cause stress and emotional eating episodes: a kitchen, a restaurant, a supermarket, an office and so on. Each of these environments could be modified by the therapist with objects and persons depending on patients' personal characteristics and needs.

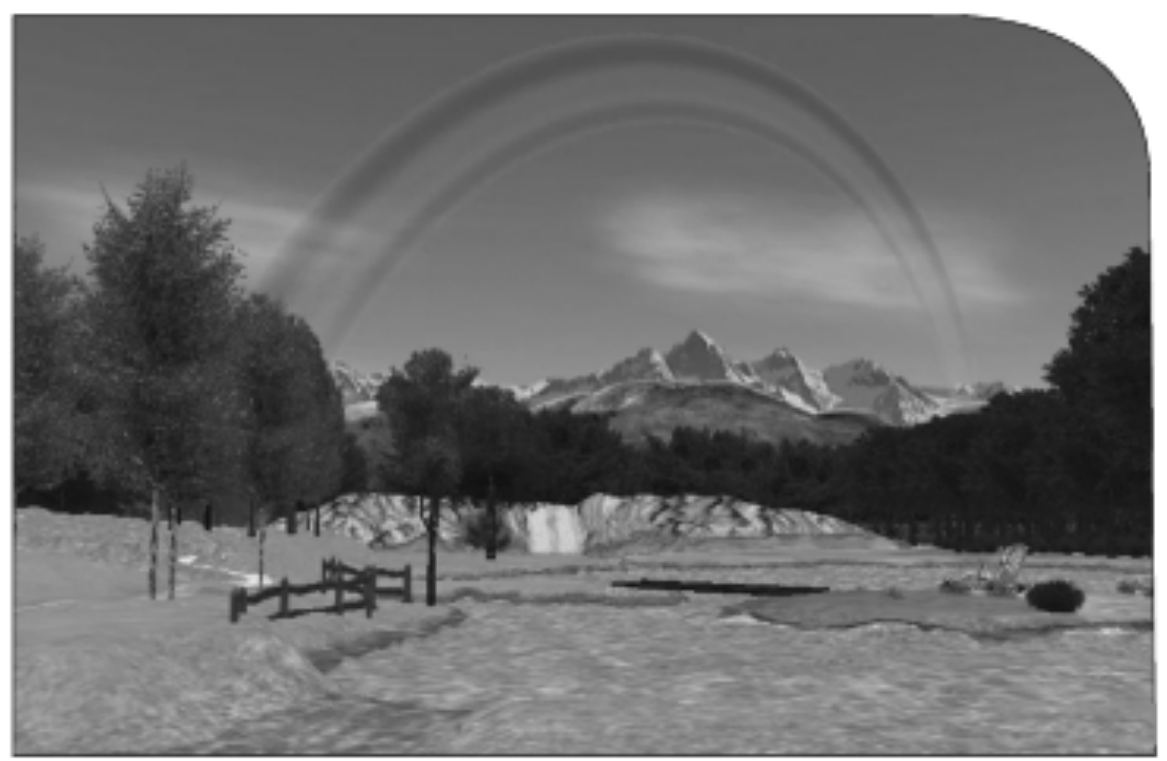

(a) 


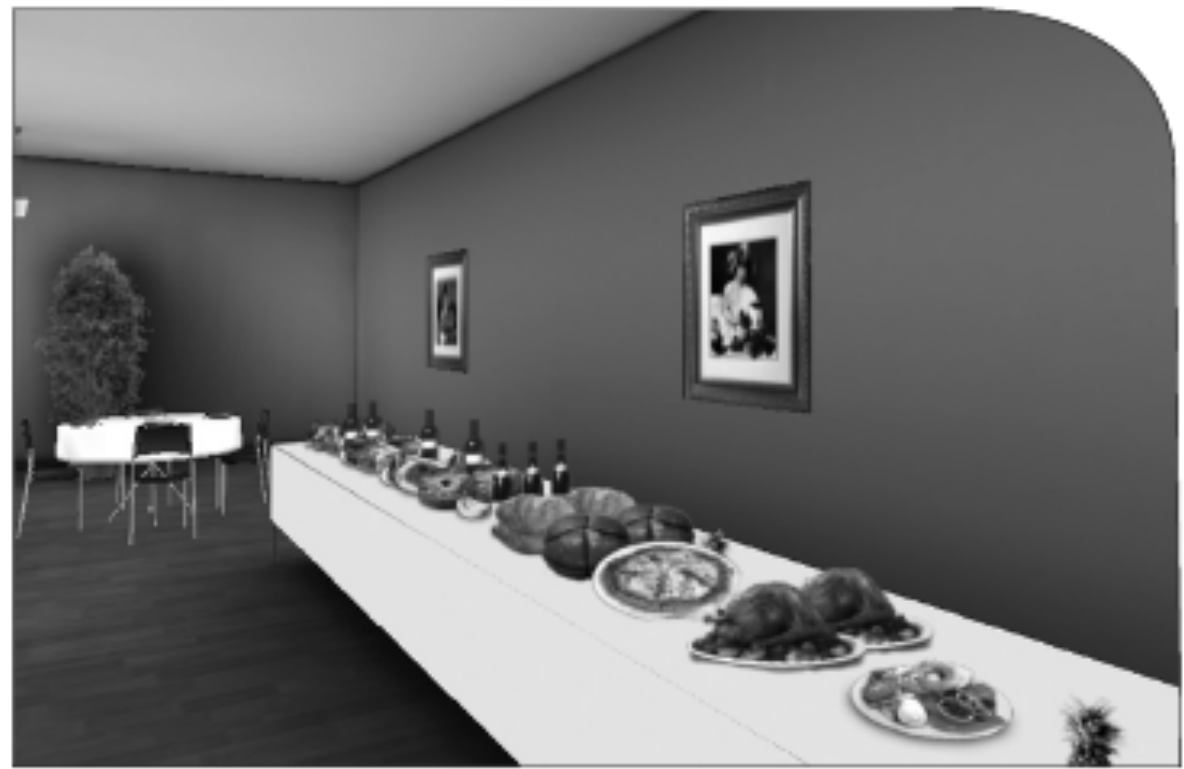

(b)

Fig. 1. Two screen shots showing (a) the Green Valley, and (b) the virtual buffet.

\section{Technological devices}

The VR system is composed by:

- a laptop (Asus G2S; Intel ${ }^{\circledR}$ Core $^{\mathrm{TM}} 2$ Extreme Processor X7800);

- an Head Mounted Display (HMD), Sony Glasstron PLM S-700, equipped with a visual device for a $3 \mathrm{D}$ view of the virtual environment and an audio device (earphones) to listen to the narratives;

- a position tracker, Intersense Intertrax $256 \mathrm{~Hz}$, that allows the user to modify his/her point of view in the virtual environment according to his/her movements in the real world;

- a joystick.

\section{Statistical Analysis}

Power analysis with alpha $=0.05$ showed a statistical power of 0.80 and a total sample size of 51 to detect a large difference $(\mathrm{f}=0.45)$ between the three groups (VR, IM and WL). Power analyses were made using GPOWER (Faul \& Erdfelder, 1992).

Normality of distributions was tested with Kolmogorov-Smirnov test, which showed the violation of the assumption for many variables in all the three groups. As noted by Hogan and Peipert (Hogan \& Peipert, 1998), when the 
variables are not normally distributed, but rather skewed in some direction or kurtosis, as in this case, it is more appropriate to compare the median than the mean. The most common statistic test for doing so is the MannWhitney U statistic, which typically requires large samples for powerful group comparisons.

Having a larger sample size $(n=60)$ and expecting to find a large difference between the two experimental groups and the WL condition, we decided to use exact non-parametrical tests with Monte Carlo estimate both for between and within groups comparisons. In statistics, an exact (significance) test is a test where all assumptions upon which the derivation of the distribution of the test statistic is based are met, as opposed to an approximate test, in which the approximation may be made as close as desired by making the sample size big enough. For example, an exact test at significance level 5\% will in the long run reject true null hypothesis exactly $5 \%$ of the time (Fisher, 1925), avoiding Type I Errors. The Monte Carlo method (Manley, 1991) provides an unbiased estimate of the exact $\mathrm{p}$ value, without the requirements of the asymptotic method. From the Mann Whitney U statistic for the pre and post treatment analysis, we calculated Cohen's $d$, in which a value of 0.20 may be interpreted as a small effect, 0.50 as a medium effect, and 0.80 and greater as a large effect (Cohen, 1988). The Kruskall- Wallis test with post hoc analysis (Siegel \& Castellan, 1992) was used for between groups comparisons of independent measures and the Wilcoxon rank-sum test was used for repeated measures. Chi-square test was used for categorical data, with $\mathrm{p}=0.05$, two-tailed. Data were analyzed using SPSS 11.0.

\section{Results}

Pre-treatment characteristics of the three groups were compared. As a check of the random assignment to conditions, Kruskal-Wallis test were carried out on all the epidemiological and clinical variables. None of the tests showed significant statistical differences between the three groups.

\section{Pre and post treatment analysis}

The Wilcoxon Rank-Sum test on the pre vs post treatment scores showed significant changes in the three groups for the WELSQ and the BDI (see table 2). 


\begin{tabular}{|c|c|c|c|c|c|c|c|c|}
\hline & Group & $\mathbf{N}$ & $\begin{array}{c}\text { Median } \\
\text { (PRE) }\end{array}$ & $\begin{array}{c}\text { chi square' } \\
\text { (PRE) }\end{array}$ & $\begin{array}{c}P \\
\text { (PRE) }\end{array}$ & $\begin{array}{l}\text { Median } \\
\text { (POST) }\end{array}$ & $\begin{array}{c}\text { chi square }^{\prime} \\
\text { (POST) }\end{array}$ & $\begin{array}{c}\mathrm{P} \\
\text { (POST) }\end{array}$ \\
\hline \multirow[t]{3}{*}{ WELSQ } & $\overline{\text { VR }}$ & 20 & $\overline{6.1}$ & 213 & 0.343 & $7.275^{\star \star}$ & 7.65 & 0.022 \\
\hline & IM & 20 & 5.85 & & & $7.775^{\star \star}$ & & \\
\hline & $\overline{W L}$ & 20 & 5.7 & & & $6.45^{\hbar \hbar}$ & & \\
\hline \multirow[t]{3}{*}{ BDI } & VR & 20 & $\overline{14.5}$ & 0.97 & 0.616 & $6.5^{\ldots \ldots \hbar}$ & 5.3 & 0.069 \\
\hline & IM & 20 & 11.5 & & & $6^{* \hbar}$ & & \\
\hline & $\overline{W L}$ & 20 & 13 & & & $11^{\text {k* }}$ & & \\
\hline
\end{tabular}

Tab. 2. Wilcoxon Rank-Sum test on the pre vs post treatment scores. (Wilcoxon Rank-Sum test between the pre and post scores: $*=\mathrm{p}<.05 ; * *=\mathrm{p}<.01 ; * * *=\mathrm{p}<.001)$.

Between groups, analyses on post-treatment scores showed significant differences between the WL group and the two treatment groups for the WELSQ (VR vs WL: $U=125, p<0.05$, effect size $d=0.81$; IM vs WL: $\mathrm{U}=101.5, \mathrm{p}<0.01$, effect size $\mathrm{d}=1.13$ ) and the BDI (VR vs WL: $\mathrm{U}=127$, $p<0.05$, effects size $d=0.78$; IM vs $W L: U=126, p<0.05$, effect size $d=0.79$ ), while no significant differences were found between the VR and IM groups (see figures 3 and 4).

With respect to weight data, median weight significantly decreased within the three groups $(\mathrm{p}<0.01)$, but no significant difference in weight reduction was found among them.

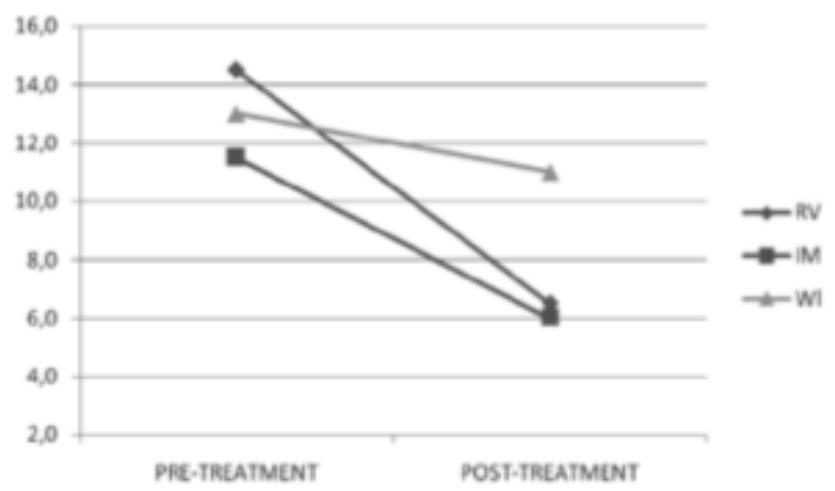

(a) 


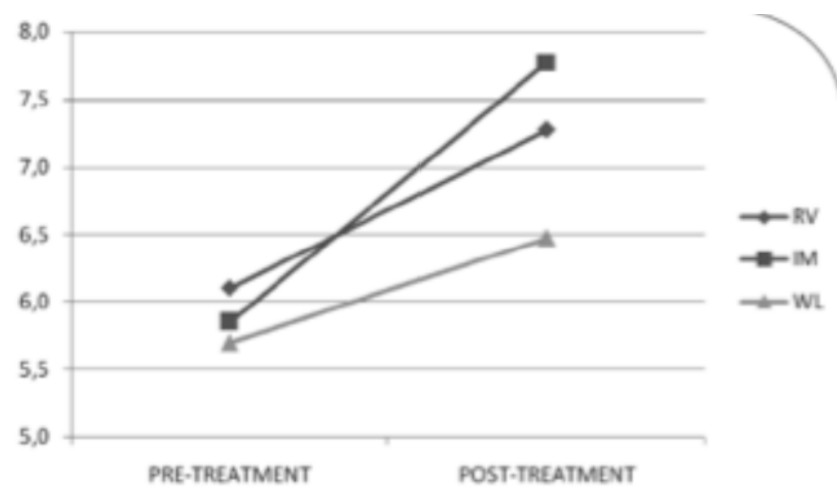

(b)

Fig. 2. (a) BDI median scores for the 3 groups before and after treatment; (b) WELSQ median scores in the 3 groups before and after treatment.

Pre- and post- sessions analysis

The Wilcoxon Rank-Sum test showed a significant decrease of state anxiety (STAI-Y1) and a significant increase of relaxation (VAS-R) before and after each treatment session both in the VR and in the IM groups, while no differences were found between them.

Accordingly to these data, we found significant decreases in heart rate values both in the VR and IM groups after each session.

\section{Discussion}

This explorative study evaluates the effectiveness of a relaxation training supported by the use of virtual environments and portable $\mathrm{mp} 3$ players in a sample of female obese inpatients with emotional eating. During the first part of the treatment, patients included in the VR group were immersed in a very relaxing virtual environment in which calm images associated to relaxing narratives helped them to reach a good level of relaxation. During the second part of the protocol, the same patients experienced some critical situations related to the maintaining/relapse mechanisms (Kitchen, Supermarket, Pub, Restaurant, etc) and were guided to react to them with the previous acquired relaxation techniques. Patients included in the IM group followed a very similar 
relaxation training in which virtual environments were replaced by imagination. In both cases, at the beginning of the treatment, participants received an $\mathrm{mp} 3$ player and were instructed to listen to the narratives anytime they felt stressed, anxious or sad (or at least once a day). These mobile devices allowed patients to practice relaxation techniques regularly, even when they did not have the possibility to meet the therapist (for example during the week-end days).

The analyses performed on the psychological variables at the beginning and at the end of the treatment indicated that the relaxation training increased the perceived self-efficacy for eating control as measured with the WELSQ and reduced the depression level as shown by the BDI scores in both the experimental groups (VR and IM). We argue that these positive results do not exclusively depend on the residential weight control treatment, since we found them only in the two experimental groups, but not in the WL group. In particular, the effectiveness of relaxation training on the perceived selfefficacy for eating control means that patients improved beliefs about being able to cope functionally with critical situations and supports the hypothesis that obese patients with emotional eating tend to use eating to cope with stressful situations and related negative emotions (Lindeman \& Stark, 2001; Ozier et al., 2008; Volkow \& Wise, 2005). These results are quite impressive since they have been obtained in only three weeks of relaxation training.

Weight significantly decreased in all the three groups, without difference among them. This result was largely expected because, during the residential treatment, food intake, physical exercise and eating behavior were strictly controlled.

Regarding the within sessions effects, the relaxation training was effective in reducing state anxiety, as measured by the STAYY1, and in increasing the relaxation level, as measured by the VAS-R, in the two groups of patients. Similar results have been found in the last three sessions when they were exposed to virtual simulated or imagined stressful situations and asked to relax using the previous acquired techniques. Coherently with the psychological observations, along the entire treatment, we observed a significant decrease in heart rate values indicating an objective reduction of the physiological arousal. Regarding the two different conditions (VR vs IM), they appear to have different effects neither on post-treatment nor on within-sessions outcomes. This result is in contradiction with Freeman's (J. Freeman et al., 2004) and Riva's findings (Riva et al., 2008) that showed that VR is more effective than the narratives alone in facilitating relaxation. A possible explanation of this apparent lack of effectiveness of VR 
immersion regards the use of a quite uncomfortable head-mounted display, of a position tracker often characterized by a poor movements accuracy and of a virtual environment not totally feasible in its technical and graphical features. Since in their study Freeman et al. (J. Freeman et al., 2004) provided immersion using a large projection screen instead of an headmounted display, we hypothesize that the HMD with an embedded position tracker, usually adopted for VR exposure, is not indicated for relaxation purposes. Moreover, the virtual environment we used is graphically less realistic compared to the one used by Freeman (J. Freeman et al., 2004) and Riva (Riva et al., 2008). Furthermore, it is characterized by few moving objects that are not strictly matched with subjects' physical rhythms (i.e. breathing and heart rate). Probably, these critical features negatively moderated the expected enhancing interaction effect of the relaxing narratives and the audio-visual virtual environment. However, this is only a speculation because we did not measure the sense of presence and thus we can say nothing about the subjective quality of experience.

In conclusion, some useful considerations emerge from this exploratory study. First, a brief relaxation training (12 sessions for 3 weeks) provided to obese female inpatients with recurrent emotional eating episodes seems to improve their perceived self-efficacy in eating control and depressive symptoms. We argue that these improvements may have a positive effect on eating behavior (i.e. reduced emotional eating episodes) and on long-term weight loss. However, we need further clinical trials in order to test this hypothesis. Second, even if we did not find any difference between the two conditions (VR and IM), we suppose that VR may play a significant role in providing relaxation when it is well technically and graphically arranged. In his book on "emotioneering" Freeman (D. Freeman, 2003) suggests some possible "recipes" for emotional induction through media, but it is not clear how to manipulate the aspects of form and content of interactive media to induce an emotional response (Riva, Mantovani et al., 2007). From the present experience we conclude that, for relaxation training purposes, providing immersion by an head-mounted display with an embedded tracker device is probably not feasible, that graphical realism is a critical concern and that key moving objects linked to the subjects' physical rhythms (i.e. breathing and heart rate) are necessary. 


\section{Acknowledgments}

The present work was supported by the Italian MIUR FIRB program (Project "Neurotiv Managed care basata su telepresenza immersiva virtuale per l'assessment e riabilitazione in neuropsicologia e psicologia clinica", RBNE01W8WH. www.neurotiv.org). 


\section{References}

1. Beck, A. T., \& Steer, R. A. (1993). Beck depression inventory. San Antonio, TX: The Psychological Corporation.

2. Bekker, M. H. J., van de Meerendonk, C., \& Mollerus, J. (2004). Effects of negative mood induction and impulsivity on self-perceived emotional eating. International Journal of Eating Disorders, 36, 461-469.

3. Blair, A. J., Lewis, V. J., \& Booth, D. A. (1990). Does emotional eating interfere with success in attempts at weight control? . J Clin Psychol., 15, 151-157.

4. Borkovec, T. D., \& Costello, E. (1993). Efficacy of applied relaxation and cognitive-behavioral therapy in the treatment of generalized anxiety disorder. J Consult Clin Psychol, 61(4), 611-619.

5. Braet, C., \& Van Strien, T. (1997). Assessment of emotional, externally induced and restrained eating behaviour in nine to twelveyear-old obese and non-obese children. Behav Res Ther, 35(9), 863873.

6. Carver, C. S., Scheier, M. F., \& Weintraub, J. K. (1989). Assessing coping strategies: A theoretically based approach. Journal of Personality and Social Psychology, 56, 267-283.

7. Clark, M. M., Abrams, D. B., Niaura, R. S., Eaton, C. A., \& Rossi, J. S. (1991). Self-efficacy in weight management. J Consult Clin Psychol, 59(5), 739-744.

8. Cohen, J. (1988). Statistical power analysis for the behavioral sciences (2nd ed.). Hillsdale, New York: Lawrence Earlbaum Associates.

9. Crowther, J. H., Sanftner, J., Bonifazi, D. Z., \& Shepherd, K. L. (2001). The role of daily hassles in binge eating. Int J Eat Disord, 29(4), 449-454.

10. Faul, F., \& Erdfelder, E. (1992). GPOWER: A priori, post-hoc and compromise power analysis for MS-DOS. Bonn: Bonn University, Dep. of psychology.

11. Forety, J. P., \& Carlos Poston II, W. S. (1998). The role of the behavioral counselor in obesity treatment. J Am Diet Assoc, 98 (suppl 2), S27-S30.

12. Freeman, D. (2003). Creating emotions in games. The craft and art of emotioneering. Indianapolis: New Riders Games.

13. Freeman, J., Lessiter, J., Keogh, E., Bond, F. W., \& Chapman, K. 
(2004). Relaxation island: virtual and really relaxing. Paper presented at the 7th International Workshop on Presence, Universitat Politecnica de Valencia, Spain.

14. Geliebter, A., \& Aversa, A. (2003). Emotional eating in overweight, normal weight, and underweight individuals. Eat Behav, 3(4), 341347.

15. Gluck, M. E. (2006). Stress response and binge eating disorder. Appetite, 46(1), 26-30.

16. Gross, J., \& Levenson, R. W. (1995). Emotion elicitation using films. Cognition and Emotion, 9, 87-108.

17. Hogan, J. W., \& Peipert, J. F. (1998). Power and sample size. Clin Obstet Gynecol, 41(2), 257-266.

18. Lindeman, M., \& Stark, K. (2001). Emotional eating and eating disorder psychopathology. Eating Disorders: The Journal of Treatment \& Prevention, 9, 251-259.

19. Masheb, R. M., \& Grilo, C. M. (2006). Emotional overeating and its associations with eating disorder psychopathology among overweight patients with binge eating disorder. International Journal of Eating Disorders, 39(2), 141-146.

20. Ong, L., Linden, W., \& Young, S. (2004). Stress management. What is it? Journal of Psychosomatic Research, 56, 133-137.

21. Ost, L. G., \& Breitholtz, E. (2000). Applied relaxation vs. cognitive therapy in the treatment of generalized anxiety disorder. Behaviour Research and Therapy, 38(8), 777-790.

22. Ozier, A. D., Kendrick, O. W., Leeper, J. D., Knol, L. L., Perko, M., \& Burnham, J. (2008). Overweight and obesity are associated with emotion- and stress-related eating as measured by the eating and appraisal due to emotions and stress questionnaire. J Am Diet Assoc, 108(1), 49-56.

23. Popkess-Vawter, S., Wendel, S., Schmoll, S., \& O’Connell, K. (1998). Overeating, reversal theory and weight cycling. West J Nurs Res, 20, 67-83.

24. Riva, G., Gaggioli, A., Villani, D., Preziosa, A., Morganti, F., Corsi, R., et al. (2007). NeuroVR: an open source virtual reality platform for clinical psychology and behavioral neurosciences. Stud Health Technol Inform, 125, 394-399.

25 Riva, G., Mantovani, F., Capideville, C. S., Preziosa, A., Morganti, F., Villani, D., et al. (2007). Affective interactions using virtual reality: the link between presence and emotions. Cyberpsychol Behav, 10(1), 45-56. 
26. Riva, G., Manzoni, G. M., Villani, D., Gaggioli, A., \& Molinari, E. (2008). Why you really it? Virtual reality in the treatment of obese emotional eaters. In J. D. Westwood, R. S. Haluck, H. M. Hoffman, G. T. Mogel, R. Phillips, R. A. Robb \& K. G. Vosburgh (Eds.), Studies in Health Technology and Informatics (Vol. 132, pp. 417419): IOS Press.

27. Riva, G., Preziosa, A., Grassi, A., \& Villani, D. (2006). Stress Management Using UMTS Cellular Phones: A Controlled Trial. In J. D. Westwood (Ed.), Medicine Meets Virtual Reality 14: IOS Press.

28. Siegel, S., \& Castellan, N. J. (1992). Nonparametric Statistics for the Behavioral Sciences. Milan: McGraw-Hill, Inc.

29. Solomon, M. (2001). Eating as both coping and stressor in overweight control. J Adv Nurs, 36, 563-573.

30. Spielberger, C. D., Gorsuch, R. L., \& Lushene, R. E. (1970). Manual for the state-trait anxiety inventory. Palo Alto, CA: Consulting Psychologists Press.

31. Timmerman, G., \& Acton, G. (2001). The relationship between basic need satisfaction and emotional eating. Issues Ment Health Nurs, 22, 691-701.

32. Troop, N. A. (1998). Eating disorders as coping strategies: a critique. European Eating Disorders Review, 6, 229-237.

33. Vincelli, F. (1999). From Imagination to Virtual Reality: The Future of Clinical Psychology. Cyberpsychol Behav, 2(3), 241-248.

34. Volkow, N., \& Wise, R. (2005). How can drug addiction help us understand obesity. Nature Neuroscience, 8(5), 555-560.

35. Waters, A., Hill, A., \& Waller, G. (2001). Bulimics' responses to food cravings: is binge-eating a product of hunger or emotional state? Behav Res Ther, 39(8), 877-886.

36. Waterworth, J. A., \& Waterworth, E. L. (2004). Relaxation Island: $A$ Virtual Tropical Paradise. Interactive Experience. Paper present ed at the Proceedings of BCS HCI 2004: Designing for Life, Leeds, UK.

37. WHO. (2006). Obesity and overweight, from:

http://www.who.int/mediacentre/factsheets/fs311/en/index.html. 



\section{CHAPTER VI}

\section{A portable immersive system as an alternative medical treatment to reduce anxiety in minor surgical operations: a randomized controlled study}

A. Gorini, J. L. Mosso, D. Mosso, E. Pineda, N. L. Ruíz, G. T. Obrador, G. Riva.

Unpublished data. 



\section{Abstract}

Excessive anxiety is a common problem for patients who undergo surgical operations. As medication treatments alone have frequently proved to be inadequate to reduce anxiety in clinical contexts, there is an increasing interest in non-invasive complementary and alternative medical therapies (CAM) that diminish psychological discomfort during pre- and post-operative phases. Being an advanced imaginative system able to induce emotional responses, and having gained recognition as a means of attenuating distress and pain during various medical procedures, virtual reality (VR) can be also considered a CAM therapy. The aim of this study was to test the efficacy of a small, portable and immersive VR system compared to a music-based relaxation approach in reducing anxiety in a sample of patients who underwent minor surgical procedures (lipoma or cyst removals). Forty-seven patients were randomly divided into three groups: virtual reality group (VR); music group (MU); and standard care (CTR). Psychological and physiological measures were recorded immediately before (T0), 45 minutes after (T1), and 90 minutes after (T2) the operation. The results indicated that the VR group showed a significant decrease of subjective anxiety at T1, while the MU group showed it at T2. No significant differences in subjective anxiety were found between T0, T1 and T2 in the CTR group. Regarding the physiological parameters, we did not find any significant difference between the beginning and the end of the operation in any of the three groups of patients.

Despite some limitations (the small number of sample and the lack of interaction with the virtual environment), this is the first study demonstrating that a portable, low cost, and easy-to-use VR system is more effective than music alone in reducing subjective anxiety caused by minor surgical interventions.

\section{Perspective}

This study suggests a possible role of a virtual reality portable system in reducing anxiety in patients who undergo ambulatory 
surgical operations. Given the limited costs of this approach, these findings should encourage surgeons to adopt it in order to help patients to face at the minor surgical procedures with a reduced level of anxiety. 


\section{Introduction}

The thought of being 'cut open', the fear of pain, and the idea that something could go wrong are common preoperative worries that lead to increased anxiety and stress in surgical patients. Preoperative anxiety (anxiety regarding impending surgical experiences) can be a major problem for patients $^{34}$ being often the cause of a great discomfort before the operation and of a number of maladaptive post surgery outcomes, including postoperative fatigue and pain, ${ }^{44}$ higher risk of surgical site infection, ${ }^{29}$ sleep disturbances, ${ }^{12}$ lack of fully postoperative recovery, ${ }^{26,43}$ and, in the worst cases, post traumatic stress disorder. ${ }^{10}$ Preoperative anxiety can also increase the intensity of pain experienced during surgery. ${ }^{35}$

As medication treatments alone have often proved to be inadequate to reduce stress and anxiety in surgical contexts, in the last decades there has been an increased interest in non-invasive complementary and alternative medical (CAM) therapies, such as music, relaxation, guided imagery, hypnosis, acupuncture, etc., that are usually acceptable to the vast majority of patients. ${ }^{28,53,54}$ Virtual reality (VR) can be considered the most technologically advanced form of CAM therapy, having gained recognition as a means of attenuating distress and pain during medical procedures. ${ }^{15} \mathrm{VR}$ provides a particularly intense form of immersive cognitive and emotional distraction that taxes the patient's limited attention capacity, resulting in the withdrawal of attention from the real, noxious, external stimuli with a subsequent reduction in perceived pain and stress. ${ }^{15,41,57}$

Preliminary evidence that entering an immersive virtual environment acts as a potent non-pharmacologic analgesic on patients undergoing daily burn wound care came from a preliminary study by Hoffman and coll. ${ }^{21}$ on a sample of adolescent burn victims. Subsequently, a series of controlled studies confirmed the efficacy of VR in reducing pain, and anticipatory and procedural anxiety in many other medication conditions such as invasive clinical procedures, including phantom limb pain, ${ }^{6}$ physical therapy in burn victims, ${ }^{19,20}$ venipuncture, ${ }^{40,56}$ paediatric intravenous placement for magnetic resonance imaging and computed tomography, ${ }^{16}$ lumbar puncture in adolescents with cancer, ${ }^{46}$ invasive medical procedures in pediatric oncology patients, ${ }^{14}$ and pre-surgical anesthesia in children. ${ }^{36}$

$\mathrm{VR}$ is also effective in reducing distress in non-painful procedures such as chemotherapy for breast cancer in adult women ${ }^{48}$ and outpatient chemotherapy in children. ${ }^{47}$ Even if Even if patients do not feel pain in these situations, reducing medical-related distress can improve their quality of 
life, increase chances for survival, and enhance their ability to adhere to treatment regimens and cope with their disease.

The efficacy of VR distraction has been also recently investigated using the cold pressure test, a classical test of pain threshold and pain tolerance. ${ }^{45}$ This study showed that VR distraction led to a significant increase in pain threshold and pain tolerance and significant decrease in pain intensity, time spent thinking about pain, and self-reported anxiety, relative to baseline. These results are in accord with the previous data showing significant improvements in both pain tolerance and pain threshold relative to their baseline scores in children exposed to active or passive distraction using a head mounted display (HMD) ${ }^{9}$ and a significant attenuation of pain-related brain activity in subjects exposed to VR during a thermal stimulation. ${ }^{23}$ Several additional studies conducted on healthy subjects undergoing experimentally induced ischemia have demonstrated that VR increases pain tolerance, $^{32,50}$ decreases self-reported pain intensity, ${ }^{22,32}$ and reduces affective unpleasantness and time spent thinking about pain. ${ }^{32}$ These results are strongly supported by recent neuroimaging data obtained by Hoffman and coll. ${ }^{24,25}$ who used subjective pain ratings and functional magnetic resonance imaging to measure pain and pain-related brain activity in subjects receiving opioid and/or VR distraction. Results showed converging evidence for the analgesic efficacy of opioid administration alone and VR distraction alone. Furthermore, patterns of pain-related brain activity support the significant subjective analgesic effects of VR distraction when used as adjunct to opioid analgesia.

However, because VR is often used in hospital settings (operating rooms, hydro tanks, patients' waiting rooms) smaller, more portable, and easy-touse immersive distractive systems are desirable, especially if researchers aspire to transform VR from a research tool to a clinical instrument. As clearly stated in Markus et al. ${ }^{33}$ actual VR systems (in particular, the authors refers to the "SnowWorld" software and its technical apparatus - Patterson and Hoffman, University of Washington) make the clinical implementation difficult because of the time commitment from the staff for equipment setup and clean prior and before each use of VR, the availability of nursing staff to assist with the system, the learning curve for operation of the VR equipment, and the cooperation of patients. As suggested by the authors, potential solutions include on-site technical support for equipment troubleshooting along with dedicated personnel to set-up equipment or the implementation of easy-to-use, portable VR devices that reduce the time spent for set-up and maintenance. 
Stemming from these observations, the aim of this study was to test the efficacy of a small, non-interactive portable virtual reality system to reduce surgical-related anxiety in a sample of patients who underwent ambulatory operations under local or regional anaesthesia. In particular, due to the proven positive effects of relaxation training ${ }^{31}$ and music

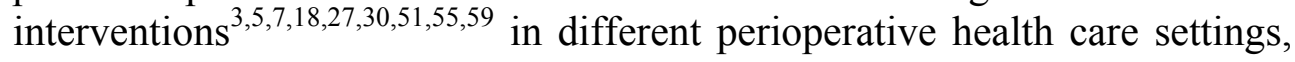
we provided patients an immersive $3-\mathrm{D}$ virtual relaxing environment accompanied by a calm music. The effect of VR exposure in reducing anxiety was compared with the effect of music alone and with a control condition in which patients did not receive any kind of distracting intervention. The hypothesis of the present study is that the immersion in a virtual reality environment accompanied by a calm music will be more effective than music alone to reduce operative anxiety.

Compared to the previous studies in which VR has been used to reduce pain and distress in different medical contexts, the present protocol presents three main innovations. First, this is the first study using VR during a surgical operation; second, the present virtual environment was created not only to distract patients, but also to relax them. A calm lake surrounded by a very quiet mountain landscape was used to induce relaxation in the patients who explored it accompanied by a relaxing music. Third, we provided the VR immersion inside the surgical room through a very small and easy-to-use system made of a portable Playstation (PSP) and a head mounted display (HMD). Being very simple to use, small, and light, this kind of equipment has the advantage of not interfering with surgical instruments or surgical settings, and requires a very short amount of time to be set up and cleaned up.

\section{Materials and Methods}

Subjects. Forty-seven patients, 19 females and 28 males, between 19 and 86 years of age participated in the study. All patients underwent a surgical operation under local or regional anaesthesia at the General and Regional Hospital No. 25 of the IMSS in Mexico City. Patients were randomly divided into three groups matched for age and gender: the Virtual Reality group (VR) $(\mathrm{N}=16 ; 6$ females and 10 males; mean age $=48.25 \pm 16.78)$; the Music group (MU) $(\mathrm{N}=15$; 6 females and 9 males; mean age $=46.73 \pm$ 13.13); and the Control group (CTR) $(\mathrm{N}=16$; 7 females and 9 males; mean age $=47 \pm 21.53)$ (fig. 1 and tab. 1). The age difference between the three 
groups was not statistically significant $(\mathrm{p}=0.97)$. Randomization process was based on the dynamic (adaptive) random allocation method ( $\operatorname{see}^{2,37}$ for a detailed description of the method).

Prior to the operation, patients in the two experimental groups (VR and MU) were instructed about the use of the HMD and the headphones, respectively. The study was approved by the Local Institutional Review Board, and all participants were asked to sign an informed consent, ${ }^{8}$ in accordance to the Declaration of Helsinki ${ }^{58}$.

\section{Flow chart diagram}

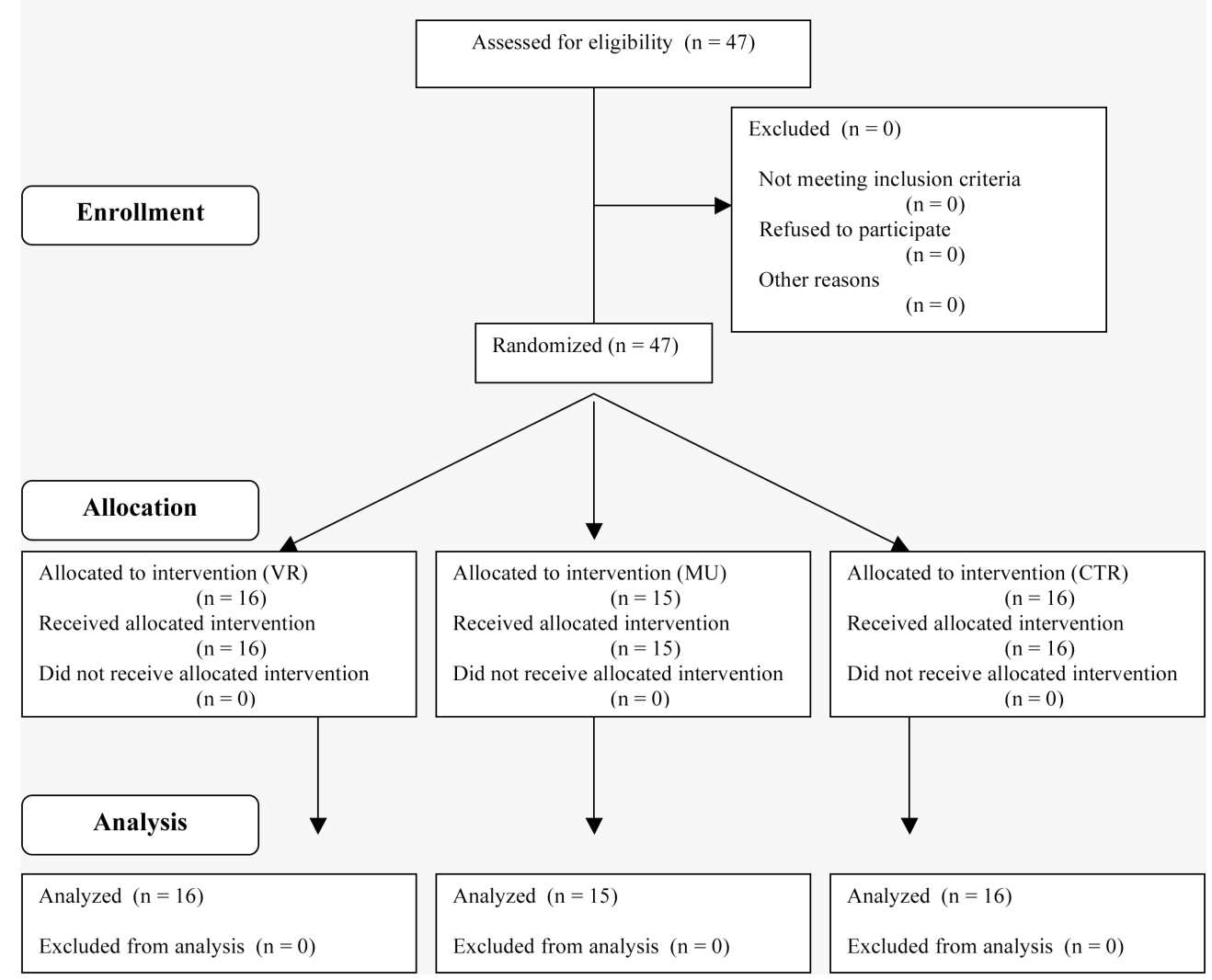

Fig. 1. Flow chart diagram representing the enrolment and allocation procedures. 
A portable VR system to reduce pre-operative anxiety

\begin{tabular}{|c|c|c|c|c|}
\hline & $\begin{array}{c}\text { VR } \\
(N=16)\end{array}$ & $\begin{array}{c}\text { MU } \\
(\mathbf{N}=\mathbf{1 5})\end{array}$ & $\begin{array}{c}\text { CTR } \\
(N=16)\end{array}$ & $\begin{array}{l}\text { TOTAL } \\
(\mathrm{N}=47)\end{array}$ \\
\hline $\begin{array}{l}\text { Age in years } \\
\text { Mean } \\
\text { Range } \\
\end{array}$ & $\begin{array}{l}48.25 \\
19-74 \\
\end{array}$ & $\begin{array}{l}46.73 \\
24-71 \\
\end{array}$ & $\begin{array}{l}47.00 \\
21-86 \\
\end{array}$ & $\begin{array}{l}47.34 \\
19-86 \\
\end{array}$ \\
\hline & $\mathbf{N}$ & $\mathbf{N}$ & $\mathbf{N}$ & $\mathbf{N}$ \\
\hline $\begin{array}{l}\text { Gender } \\
\text { Males } \\
\text { Females }\end{array}$ & $\begin{array}{c}10 \\
6 \\
\end{array}$ & $\begin{array}{l}9 \\
6 \\
\end{array}$ & $\begin{array}{l}9 \\
7 \\
\end{array}$ & $\begin{array}{l}28 \\
19 \\
\end{array}$ \\
\hline $\begin{array}{l}\text { Diagnosis } \\
\text { Cyst } \\
\text { Lipoma } \\
\end{array}$ & $\begin{array}{l}8 \\
8 \\
\end{array}$ & $\begin{array}{l}9 \\
6 \\
\end{array}$ & $\begin{array}{c}10 \\
6 \\
\end{array}$ & $\begin{array}{l}27 \\
20 \\
\end{array}$ \\
\hline $\begin{array}{l}\text { Anaesthesia } \\
\text { Local } \\
\text { Regional }\end{array}$ & $\begin{array}{c}12 \\
4\end{array}$ & $\begin{array}{c}12 \\
3\end{array}$ & $\begin{array}{l}8 \\
8\end{array}$ & $\begin{array}{l}32 \\
15\end{array}$ \\
\hline
\end{tabular}

Tab. 1. Demographic, clinical, and procedural characteristics of the sample.

Surgical interventions. Operations consisted of small lipoma or cyst removals and were performed under local or regional anaesthesia (see Table 1 for details). To remove cysts or lipomas, the doctor administers the anaesthesia in the area around them and makes an incision in the skin in order to remove the growth. Usually, this is known to be a non-painful procedure.

Operations reported in the present study typically lasted about 90 minutes and all the patients were discharged from the hospital the same day in which the operation was performed. The duration of operations and the length of hospital stay did not differ between patients.

Technical equipment. The Playstation Portable (PSP) is a Sony handheld game console that measures approximately $17 \times 7.3 \times 2.2 \mathrm{~cm}(6.7 \times 2.9 \times$ 0.9 in), and weighs 280 grams (9.88 ounces). The front of the console is dominated by the system's $11 \mathrm{~cm}$ (4.3 in) LCD screen, which is capable of $480 \times 272$ pixel video playback with 16.77 million colours. The PSP includes an $1800 \mathrm{mAh}$ battery that will provide about 4-6 hours of gameplay, 4-5 hours of video playback, or 8-11 hours of audio playback. The PSP was connected to the HMD Vuzix iWear AV 920, a high resolution head mounted display, in order to allow the patients a $3 \mathrm{D}$ immersion in the virtual environment. The iWear weighs only 2.9 ounces, 
pivots up to 15 degrees for comfortable viewing angle and has integrated speakers that allow the user to plug in his/her own headsets.

$V R$ condition. The Green Valley, ${ }^{42}$ a relaxing environment showing a mountain landscape around a calm lake, is presented to the patient together with relaxing music and soft sounds (bird songs, sounds of water flowing, etc.) (Fig. 2). Patients can virtually sit on a comfortable deck chair and observe the natural scene, which can help them feel relaxed. Unlike some virtual reality systems, The Green Valley is immersive but non-interactive, meaning that users are able to see the virtual reality environment as if they are actually in the environment, but are unable to move around or otherwise interact with the environment. Wearing the HMD, patients were immersed in the virtual environment for the entire length of the operation.

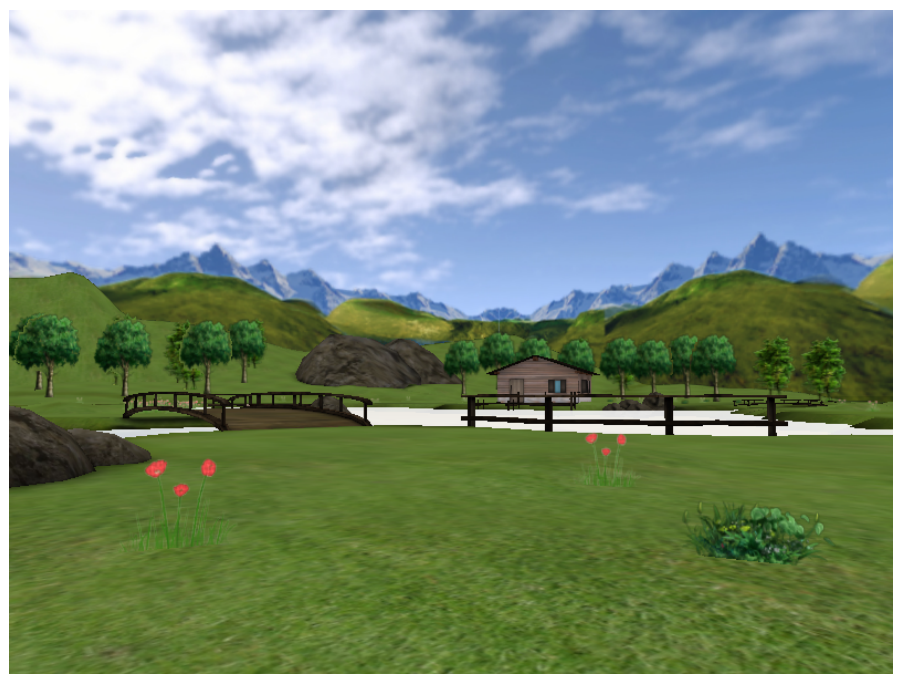

Fig. 2. A screenshot of the Green Valley, the virtual environment used to help patients to become relaxed during the surgical operation.

Music condition. Relaxing music and nature sounds were used to provide a calm atmosphere and reduce stress. In the VR group music accompanied the virtual environment, while in the MU group it was provided to the patients through earphones without any visual stimulation.

Control condition (standard care). Patients in the CTR condition did not received any kind of complementary treatment. 
Experimental procedure. Patients in the two experimental groups wore the HMD and the headphones connected to the PSP (VR group) (fig. 3) or the earphones alone (MU group) few minutes before the anaesthetic injection. The total length of the virtual relaxation session was 90 minutes, corresponding, more or less, to the duration of the intervention.

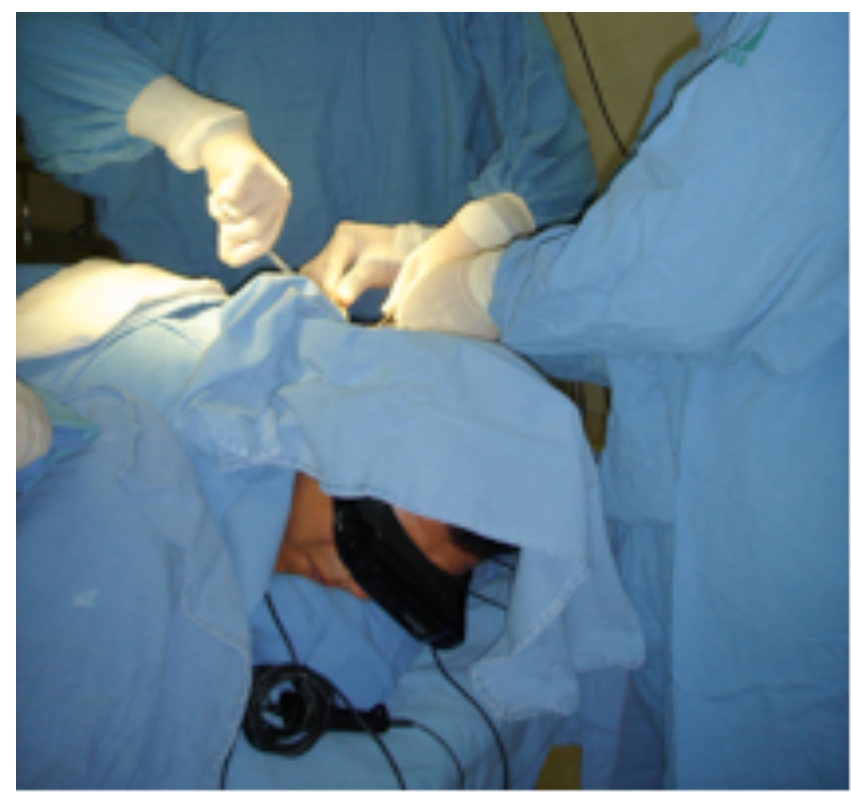

Fig. 3. A patient included in the VR group wearing the HMD during the operation. She is immersed in the virtual environment being isolated from the threatening surgical context.

Subjective measure of anxiety. Patients' self-ratings of anxiety served as primary dependent variables. Ratings were administered immediately before (T0), after 45 minutes (T1) and after 90 minutes of operation (T2). Measurements at T1 and T2 were taken during a brief (approximately 2 minute) pause in the operation. Patients gave their ratings using the visual analogue scale for anxiety (VAS-A). The VAS-A is 0 to 10 scale, where 0 indicates "no anxiety" and 10 "anxiety as bad as it could possibly be". The VAS-A is a widely used anxiety measurement with well-established validity and reliability. ${ }^{1,11,38,39,52}$ Among the numerous tools available for assessing anxiety, direct scaling procedures, such as VAS, are popular because of their simplicity, versatility, relative insensitivity to bias effects, and the assumption that the procedures yield numerical values that are valid, reliable, and on a ratio scale. ${ }^{38}$ 
Physiological measurements. In the operating room, patients were attached to the electrocardiograph, noninvasive blood pressure, and pulse oximeter (SpO2) (Datex-Ohmeda S/5, Finland) so that heart rate, blood pressure and saturation were continuously recorded. The mean values corresponding to 3 minutes of registration at $\mathrm{T} 0, \mathrm{~T} 1$ and $\mathrm{T} 2$ were used as dependent variables.

Data analysis. Data were entered into Microsoft ${ }^{\circledR}$ Excel and analyzed using SPSS ${ }^{\circledR}$, version 12 . Two-tailed $p$ values of less than 0.05 were considered to be significant. Repeated measures analysis of variance (ANOVAs) were conducted to test for the differences in the VAS-A scores, heart rate, saturation and blood pressure within groups, and for the between groups effects on the psychological and physiological variables. Two-way mixed model analyses of variance (time by group) with VAS-A score, heart rate, saturation and blood pressure values as dependent variables were used to test main and interaction effects.

\section{Results}

Subjects in the three groups were statistically comparable based on demographical, clinical and procedural characteristics (see tab 1).

$V A S-A$ scores. The level of subjective anxiety was compared between the three groups of patients at T0, T1 and T2. The repeated measures ANOVAs revealed a significant main effect of time $(F[2,88]=15.14, \mathrm{p}<0.01)$ and a significant time $x$ group interaction $(F[1,44]=6.75, p<0.05)$. In particular, post-hoc analyses showed significant differences between VR and MU groups $(\mathrm{p}=0.007)$ and VR and CTR groups $(\mathrm{p}=0.002)$ at T1 and VR and CTR groups $(p=0.005)$ and MU and CTR groups $(p=0.028)$ at T2. No significant differences were found at T0 between the three groups. Post-hoc analyses with two-tailed paired-samples t-tests comparing VAS values at T0 and $\mathrm{T} 2$ also showed that the subjective anxiety significantly decreased in the $\operatorname{VR}(\mathrm{t}(15)=3.61, p=0.003)$ and in the MU group $(\mathrm{t}(14)=4.70, p=0.000)$, but not in the CTR group $(\mathrm{t}(15)=2.02, p=0.080)$ (fig. 4). 
A portable VR system to reduce pre-operative anxiety

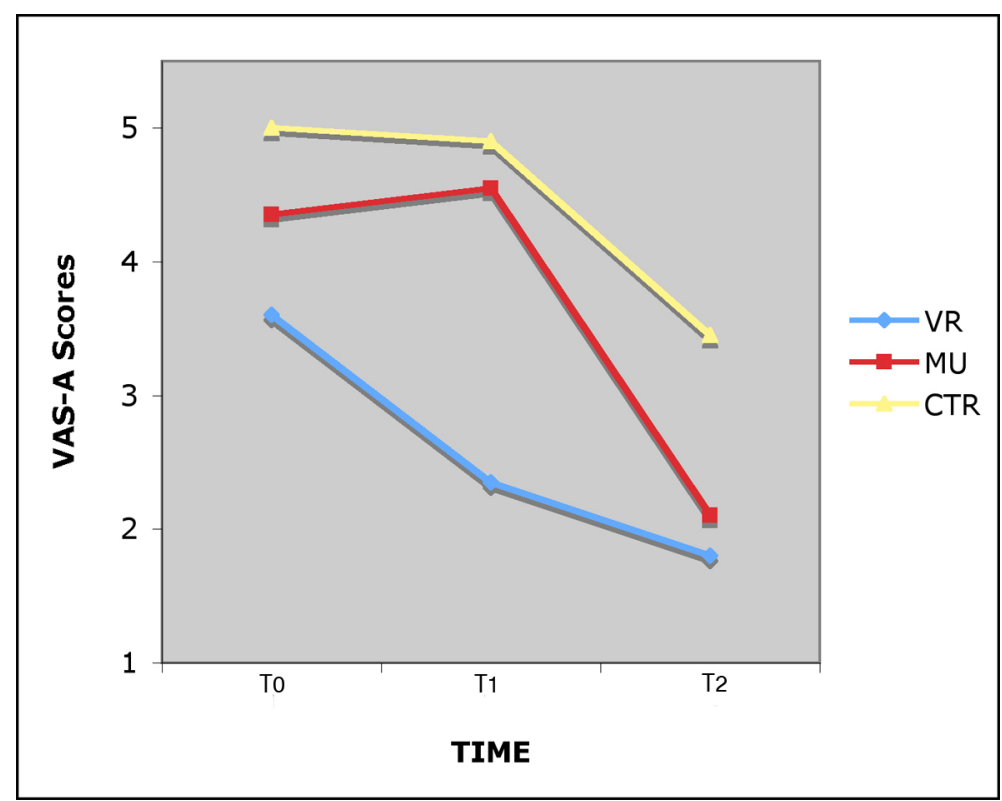

Fig. 4. VAS-A scores obtained from the 3 groups of patients at T0, T1 and T2.

Physiological measurements. Heart rate, saturation and blood pressure values were recorded as physiological measures of anxiety, and compared between the three groups of patients at T0, T1 and T2 using repeated measures ANOVAs. No significant differences were found in any of these measurements.

Although not statistically significant $(\mathrm{F}[2,88]=1.87, \mathrm{p}=0.162)$, heart rate had a tendency to decrease from $\mathrm{T} 0$ to $\mathrm{T} 2$ in the two experimental groups (mean VR at T0 $=78.50 \pm 11.48$; mean VR at $\mathrm{T} 2=73.81 \pm 10.85$; mean $\mathrm{MU}$ at T0 $=77.07 \pm 16.42$; mean MU at T2 $=72.87 \pm 8.65)$, but not in the CTR group (mean CTR at T0 $=74.88 \pm 13.99$; mean CTR at T2 $=74.19 \pm$ 11.78).

\section{Discussion}

The results of this study show that a non-interactive immersive virtual reality environment decreases the level of subjective anxiety sooner than relaxing music alone, even if both these distraction techniques are effective in the long term. In fact, the VAS-A scores indicate that, in the first 45 minutes anxiety significantly decreases in the VR group, remaining high in 
the other two groups. After 90 minutes, the level of anxiety is significantly lower both in the VR and MU groups, but not in the CTR group. These results support the efficacy of virtual reality as a distraction technique that reduces anxiety in minor surgical operations. Its findings are in line with previous studies using VR as an alternative medical treatment for various medical conditions. ${ }^{4,13,14,16,48}$

Regarding the physiological measurements, although no significant differences were found in physiological parameters from T0 and T2 between the three groups, heart rate did have a tendency to decrease in the VR and MU groups, but not in the CTR group. This lack of statistically significant differences indicates that reduction of subjective anxiety is not necessarily associated with a corresponding decrease in physiological parameters. This finding can be explained at least in two ways. First, heart rate, saturation and blood pressure were almost at normal level at the beginning of the study, thus changes could not be large, clinically or statistically relevant. An alternative explanation could be that while distraction is efficacious in reducing subjective sensation of anxiety, it does not really influence the individuals' physical state.

In many perioperative situations in which patients experience significant levels of anxiety despite the administration of standard therapies such as conscious sedation and local anaesthetics, the development of an anxiolytic with minimal psychomotor impairments and no side effects is desirable. The immersion in a relaxation environment is a non-invasive experience, which helps patients to cope with preoperative anxiety by providing them with an attractive, immediate alternative to their excessive worries. Compared to music alone, that is also a commonly used distraction technique, $5,18,27,51,55,59$ a non-interactive VR environment that visually immerses patients in a peaceful world, helps them become easily relaxed in a shorter period of time. Moreover, wearing a HMD that isolates the patient from the surgical context represents a great advantage for those who are particularly scared of medical procedures.

We also hypothesized that during minor surgical procedures, as the ones considered in the present study, the use of VR might result in a general reduction of costs for both the individual and the health care system. In particular, by decreasing the level of operative anxiety, VR could reduce the use of anxiolytic drugs and the time spent for post-operative recovery, thus improving the quality of the patient's experience. Moreover, the results of the present study show that a portable, easy to manage and relatively inexpensive VR system is safe and effective in ameliorating anxiety experienced in ambulatory surgical procedures. By using this kind of 
portable, easy to use equipment, a patient's immersion in the virtual experience would have fewer costs (VR set-up and clean-up time, and technical preparation of the staff members) than what is usually required by other VR systems. ${ }^{33}$

Future studies focusing on these speculative observations should be performed in order to determine the costs and benefits of this innovative approach.

The main limitations of this study are the relatively small number of patients per group and the lack of interaction with the virtual environment. Although, the total number of patients in the study is large, the number of patients per group is small. Moreover, in order to accurately evaluate the effect of VR compared to other distraction techniques, we considered it necessary to have not only a control group who did not receive any kind of extra treatment, but also a sample that received a different distraction intervention (i.e. music alone). Regarding the lack interaction between the patient and the virtual environment, the limitation is minimal based on Sherman and Craig's definition ${ }^{49}$ of a virtual world. Sherman and Craig deemed a virtual world to be "an imaginary space often manifested through a medium" (pg. 7), and the virtual experience provided in the present study fits this definition. The Green Valley is a 3-D generated virtual world, which includes a number of selected virtual objects able to provide emotional induction. The efficacy of the Green Valley as a non-interactive virtual environment able to modify emotional experience was recently verified in a controlled study ${ }^{17}$ in which it was compared with traditional passive videos. Researchers found that only the Green Valley virtual environment was able to reduce the anxiety level in stressed subjects.

Nevertheless, in order to further improve the positive effects of virtual reality in reducing anxiety in operative settings, the next step should be the development of an interactive portable virtual reality system that allows patients to interact with the environment using an external game device (i.e. a gamepad) or, perhaps, the accelerometer integrated in the new generation of mobile phones (such as IPhone). In fact, although different kinds of distraction conditions are effective in reducing anxiety, the interactive distraction has been demonstrated to be significantly more effective than the others. ${ }^{9}$ Finally, because one possible explanation for the efficacy of the VR can be attributed to the visual occlusion provided by the HMD, future studies should control for this variable by creating visual occlusion in all experimental conditions. This would enable investigators to more confidently attribute significant results to the VR itself and not simply to the fact that the patient cannot see the medical procedure. 
In conclusion, this study highlights the advantages of using a relaxing VR system to reduce subjective anxiety commonly experienced by patients who undergo minor surgical operations. Due to the high frequency of these procedures in clinical practice, our suggestion is to improve the VR-based approach in order to obtain an easy-to-use, non-pharmacologic adjunct to conventional standards of care in order to reduce anxiety and possibly reduce costs associated with minor surgical procedures.

\section{Acknowledgments}

The present work was supported by the Italian MIUR FIRB program (Project "IVT2010 - Immersive Virtual Telepresence (IVT) for Experiential Assessment and Rehabilitation - RBIN04BC5C) - and by the European Union IST Program (Project "INTREPID - A Virtual Reality Intelligent Multi-sensor Wearable System for Phobias' Treatment" - IST-2002507464). 


\section{References}

1. Abu-Saad H, Holzemer WL: Measuring children's self-assessment of pain. Issues Compr Pediatr Nurs 5:337-349, 1981.

2. Beller EM, Gebski V, Keech C: Randomisation in clinical trials. The Medical Journal of Australia 177:565-567, 2002.

3. Berbel P, Moix J, Quintana S: [Music versus diazepam to reduce preoperative anxiety: a randomized controlled clinical trial]. Rev Esp Anestesiol Reanim 54:355-358, 2007.

4. Chan EA, Chung JW, Wong TK, Lien AS, Yang JY: Application of a virtual reality prototype for pain relief of pediatric burn in Taiwan. J Clin Nurs 16:786-793, 2007.

5. Chetta HD: The effect of music and desensitization on preoperative anxiety in children. J Music Ther 18:74-87, 1981.

6. Cole J, Crowle S, Austwick G, Henderson Slater D: Exploratory findings with virtual reality for phantom limb pain; from stump motion to agency and analgesia. Disabil Rehabil:1-9, 2009.

7. Cooke M, Holzhauser K, Jones M, Davis C, Finucane J: The effect of aromatherapy massage with music on the stress and anxiety levels of emergency nurses: comparison between summer and winter. $\mathrm{J}$ Clin Nurs 16:1695-1703, 2007.

8. Council for International Organizations of Medical Sciences. International ethical guidelines for biomedical research involving human subjects. In: CIOMS. Geneva, 2002.

9. Dahlquist LM, McKenna KD, Jones KK, Dillinger L, Weiss KE, Ackerman CS: Active and passive distraction using a head-mounted display helmet: effects on cold pressor pain in children. Health Psychol 26:794-801, 2007.

10. de Jongh A, Olff M, van Hoolwerff H, Aartman IH, Broekman B, Lindauer R, Boer F: Anxiety and post-traumatic stress symptoms following wisdom tooth removal. Behav Res Ther 46:1305-1310, 2008.

11. Downie WW, Leatham PA, Rhind VM, Wright V, Branco JA, Anderson JA: Studies with pain rating scales. Annals of the Rheumatic Diseases 37:378-381, 1978.

12. Gallagher R, McKinley S, Dracup K: Post discharge problems in women recovering from coronary artery bypass graft surgery. Aust Crit Care 17:160-165, 2004. 
13. Gershon J, Zimand E, Lemos R, Rothbaum BO, Hodges L: Use of virtual reality as a distractor for painful procedures in a patient with pediatric cancer: a case study. Cyberpsychol Behav 6:657-661, 2003.

14. Gershon J, Zimand E, Pickering M, Rothbaum BO, Hodges L: A pilot and feasibility study of virtual reality as a distraction for children with cancer. J Am Acad Child Adolesc Psychiatry 43:12431249, 2004.

15. Gold JI, Belmont KA, Thomas DA: The neurobiology of virtual reality pain attenuation. Cyberpsychol Behav 10:536-544, 2007.

16. Gold JI, Kim SH, Kant AJ, Joseph MH, Rizzo AS: Effectiveness of virtual reality for pediatric pain distraction during i.v. placement. Cyberpsychol Behav 9:207-212, 2006.

17. Grassi A, Gaggioli A, Riva G: The green valley: the use of mobile narratives for reducing stress in commuters. Cyberpsychol Behav 12:155-161, 2009.

18. Haun M, Mainous RO, Looney SW: Effect of music on anxiety of women awaiting breast biopsy. Behav Med 27:127-132, 2001.

19. Hoffman HG, Patterson DR, Carrougher GJ: Use of virtual reality for adjunctive treatment of adult burn pain during physical therapy: a controlled study. Clin J Pain 16:244-250, 2000.

20. Hoffman HG, Patterson DR, Carrougher GJ, Sharar SR: Effectiveness of virtual reality-based pain control with multiple treatments. Clin J Pain 17:229-235, 2001.

21. Hoffman HG, Doctor JN, Patterson DR, Carrougher GJ, Furness TA: Virtual reality as an adjunctive pain control during burn wound care in adolescent patients. Pain 85:305-309, 2000.

22. Hoffman HG, Garcia-Palacios A, Kapa V, Beecher J, Sharar SR: Immersive virtual reality for reducing experimental ischemic pain. International Journal of Human-Computer Interaction 15:469-486, 2003.

23. Hoffman HG, Richards TL, Coda B, Bills AR, Blough D, Richards AL, Sharar SR: Modulation of thermal pain-related brain activity with virtual reality: evidence from fMRI. Neuroreport 15:1245-1248, 2004.

24. Hoffman HG, Richards TL, Bills AR, Van Oostrom T, Magula J, Seibel EJ, Sharar SR: Using FMRI to study the neural correlates of virtual reality analgesia. CNS Spectr 11:45-51, 2006.

25. Hoffman HG, Richards TL, Van Oostrom T, Coda BA, Jensen MP, Blough DK, Sharar SR: The analgesic effects of opioids and immersive virtual reality distraction: evidence from subjective and 
functional brain imaging assessments. Anesth Analg 105:1776-1783, 2007.

26. Kagan I, Bar-Tal Y: The effect of preoperative uncertainty and anxiety on short-term recovery after elective arthroplasty. J Clin Nurs 17:576-583, 2008.

27. Kain ZN, Wang SM, Mayes LC, Krivutza DM, Teague BA: Sensory stimuli and anxiety in children undergoing surgery: a randomized, controlled trial. Anesth Analg 92:897-903, 2001.

28. Kshettry VR, Carole LF, Henly SJ, Sendelbach S, Kummer B: Complementary alternative medical therapies for heart surgery patients: feasibility, safety, and impact. Ann Thorac Surg 81:201205, 2006.

29. Levandovski R, Ferreira MB, Hidalgo MP, Konrath CA, da Silva DL, Caumo W: Impact of preoperative anxiolytic on surgical site infection in patients undergoing abdominal hysterectomy. Am J Infect Control 36:718-726, 2008.

30. Lopez V: Commentary on Lee D, Henderson A and Shum D (2004) The effect of music on preprocedure anxiety in Hong Kong Chinese day patients. Journal of Clinical Nursing 13, 297-303. J Clin Nurs 14:278-279, 2005.

31. Lovas JG, Lovas DA: Rapid relaxation--practical management of preoperative anxiety. Tex Dent J 124:1120-1125, 2007.

32. Magora F, Cohen S, Shochina M, Dayan E: Virtual reality immersion method of distraction to control experimental ischemic pain. Isr Med Assoc J 8:261-265, 2006.

33. Markus LA, Willems KE, Maruna CC, Schmitz CL, Pellino TA, Wish JR, Faucher LD, Schurr MJ: Virtual reality: Feasibility of implementation in a regional burn center. Burns, 2009.

34. Mitchell M: Patient anxiety and modern elective surgery: a literature review. J Clin Nurs 12:806-815, 2003.

35. Muglali M, Komerik N: Factors related to patients' anxiety before and after oral surgery. J Oral Maxillofac Surg 66:870-877, 2008.

36. Patel A, Tran MCJ, Schieble T, Davidson M, Schoenberg C, Bennet $\mathrm{H}$, Delphin E. Preanesthetic distraction with a Game Boy ${ }^{\mathrm{TM}}$ is more effective than midazolam in controlling pediatric anxiety: a randomized controlled study. 58th Postgraduate Assembly in Anesthesiology, New York, 2004.

37. Pocock S: Clinical trials. A practical approach. New York, John Wiley, 1984. 
38. Price DD, McGrath PA, Rafii A, Buckingham B: The validation of visual analogue scales as ratio scale measures for chronic and experimental pain. Pain 17:45-56, 1983.

39. Reading AE: A comparison of pain rating scales. J Psychosom Res 24:119-124, 1980.

40. Reger GM, Rizzo AA, Buckwalter JG, Gold J, Allen R, Augustine $\mathrm{R}$, Mendelowitz E. Effectiveness of virtual realty for attentional control to reduce children's pain during venipuncture. 2nd International Workshop in Virtual Rehabilitation, 2003.

41. Riva G. Being-in-the-world-with: presence meets social and cognitive neuroscience. In: From communication to presence: cognition, emotions and culture towards the ultimate communicative experience. Festschrift in honor of Luigi Anolli, edited by Riva G, Anguera MT, Wiederhold BK and Mantovani F. Amsterdam: IOS Press, 2006, p. 47-80.

42. Riva G, Gaggioli A, Villani D, Preziosa A, Morganti F, Corsi R, Faletti G, Vezzadini L: NeuroVR: an open source virtual reality platform for clinical psychology and behavioral neurosciences. Stud Health Technol Inform 125:394-399, 2007.

43. Rosenberger PH, Jokl P, Ickovics J: Psychosocial factors and surgical outcomes: an evidence-based literature review. J Am Acad Orthop Surg 14:397-405, 2006.

44. Rubin GJ, Hardy R, Hotopf M: A systematic review and metaanalysis of the incidence and severity of postoperative fatigue. J Psychosom Res 57:317-326, 2004.

45. Rutter CE, Dahlquist LM, Weiss KE: Sustained efficacy of virtual reality distraction. J Pain 10:391-397, 2009.

46. Sander Wint S, Eshelman D, Steele J, Guzzetta CE: Effects of distraction using virtual reality glasses during lumbar punctures in adolescents with cancer. Oncol Nurs Forum 29:E8-E15, 2002.

47. Schneider SM, Workman ML: Virtual reality as a distraction intervention for older children receiving chemotherapy. Pediatr Nurs 26:593-597, 2000.

48. Schneider SM, Prince-Paul M, Allen MJ, Silverman P, Talaba D: Virtual reality as a distraction intervention for women receiving chemotherapy. Oncol Nurs Forum 31:81-88, 2004.

49. Sherman WR, Craig A: Understanding Virtual Reality: Interface, Application, and Design. San Francisco, CA, Morgan Kaufmann, 2002. 
50. Tse MM, Ng JK, Chung JW, Wong TK: The application of eyeglass displays in changing the perception of pain. Stud Health Technol Inform 85:532-535, 2002.

51. Updike PA, Charles DM: Music Rx: physiological and emotional responses to taped music programs of preoperative patients awaiting plastic surgery. Ann Plast Surg 19:29-33, 1987.

52. Vessey JA, Carlson KL, McGill J: Use of distraction with children during an acute pain experience. Nurs Res 43:369-372, 1994.

53. Wang SM, Peloquin C, Kain ZN: Attitudes of patients undergoing surgery toward alternative medical treatment. J Altern Complement Med 8:351-356, 2002.

54. Wang SM, Caldwell-Andrews AA, Kain ZN: The use of complementary and alternative medicines by surgical patients: a follow-up survey study. Anesth Analg 97:1010-1015, table of contents, 2003.

55. Wang SM, Kulkarni L, Dolev J, Kain ZN: Music and preoperative anxiety: a randomized, controlled study. Anesth Analg 94:14891494, 2002.

56. Windich-Biermeier A, Sjoberg I, Dale JC, Eshelman D, Guzzetta $\mathrm{CE}$ : Effects of distraction on pain, fear, and distress during venous port access and venipuncture in children and adolescents with cancer. J Pediatr Oncol Nurs 24:8-19, 2007.

57. Wismeijer AA, Vingerhoets AJ: The use of virtual reality and audiovisual eyeglass systems as adjunct analgesic techniques: a review of the literature. Ann Behav Med 30:268-278, 2005.

58. World Medical Association: Declaration of Helsinki. 2000.

59. Yung PM, Chui-Kam S, French P, Chan TM: A controlled trial of music and pre-operative anxiety in Chinese men undergoing transurethral resection of the prostate. J Adv Nurs 39:352-359, 2002. 



\section{CHAPTER VII}

\section{Emotional response to virtual reality exposure across different cultures: the role of the attribution process}

A. Gorini, J. L. Mosso, D. Mosso, E. Pineda, N. L. Ruíz, M. Ramìez, J. L. Morales, G. Riva.

CyberPsychology and Behavior. 2009, Dec; 12(6):699-705. 



\section{Abstract}

Many studies have shown the ability of media - television, movies, and virtual reality (VR) experiences - to elicit emotions. Nevertheless, it is still unclear how the different factors involved user related and medium related - play a role in producing an emotional response during a VR experience.

In this paper we will investigate this issue, analyzing the role played by the cultural and technological background of the users in the emotional responses to VR. Specifically, we will use the "core affect" model of emotions developed by Russell (2003) to explore how these factors influence the way in which subjects experience virtual worlds.

Our sample includes 20 Mexican patients: 8 living in El Tepeyac, a small rural and isolated Mexican village characterized by a very primitive culture, and 12 high civilized inhabitants of Mexico City. The "Green Valley", a non interactive, immersive relaxing environment showing a mountain landscape around a calm lake was used to induce relaxation in the two groups of patients during an ambulatory surgical operation. To investigate the effects of VR on the relaxation process we measured the physiological (heart rate) and the emotional (VAS-A) patients' response before, during and after the operation.

The results show that VR was able to significantly modify the core affect (reduced arousal) in all subjects, but that the final emotional response produced by this change was influenced by the attribution process: the civilized inhabitants of Mexico City who were able to attribute the reduced arousal to the VR experience, reported a significant reduction in the self reported level of anxiety, while people from El Tepeyac showed a reduction in their physiological reactions, but not in their perceived anxiety. 


\section{Introduction}

Many studies have shown the ability of media - films, TV programs and virtual reality (VR) experiences - to elicit emotions ${ }^{1-7}$. In particular, the characteristics of a virtual reality experience - the high level of control of the interaction, and the enriched experience provided to the user - transform it in an advanced imaginative system ${ }^{8}$ : an experiential form of imagery that is as effective as reality in inducing emotional responses. This feature has been extensively used for clinical purposes ${ }^{9,10}$. On one side, VR has been used as a new medium for exposure therapy in the treatment of anxiety disorders ${ }^{9}$. The rationale is simple: in VR the patient is intentionally confronted with the feared stimuli while allowing anxiety to attenuate. Avoiding dreaded situations that reinforce specific phobias, a series of controlled exposures reduces the anxiety through the processes of habituation and extinction. VR has been also used as distraction technique in different clinical contexts in which the subject's attention to the medical procedures can increase his/her stress level and/or the perceived pain ${ }^{11-13}$. As demonstrated by different experimental studies, VR has a positive effects in attenuating pain ${ }^{14-24}$ and anxiety ${ }^{14,25,26}$ before and during different kinds of medical interventions.

Recently, Riva and colleagues ${ }^{5}$ affirmed that virtual environments can be considered as "affective media" being effective in inducing moderately to intense emotional, behavioural, and physiological responses coherent with the contents of the experienced environment; and providing a good context for assessing those dynamic changes in emotional responses. Nevertheless, even if some authors suggested possible "recipes" 27,28 , it is still unclear how the different factors involved ${ }^{29-31}$ - user related and medium related - play a role in producing an emotional response during a VR experience.

In this paper we will investigate this issue, analyzing the role of the cultural and technological background of the users in the emotional responses to VR. Specifically, we will try to explore how these factors influence the way in which the users experience virtual worlds. As a starting point of our analysis, we will use the "core affect" model of emotions developed by James Russell ${ }^{32}$ (Tab. 1). This theory refuses the traditional dichotomy that only accepts the existence of emotions and non-emotions. More, it considers the complexity of the human experiences, as well as the individual and cultural differences, in the creation of different prototypical emotional episodes. According to this author, the "core affect" is a neuropsychological category corresponding to the combination of valence and arousal levels 
that endow the emoters with a kind of "core knowledge" about the emotional features of objects and events.

\begin{tabular}{|l|l|}
\hline Term & Definition \\
\hline Core affect & $\begin{array}{l}\text { A neurophysiological state that is consciously accessible as a } \\
\text { simple, non-reflective feeling that is an integral blend of hedonic } \\
\text { (pleasure-displeasure) and arousal. Core affect responds to virtual } \\
\text { reality experiences. }\end{array}$ \\
\hline Affective quality & The ability to cause a change in core affect. \\
\hline Attributed affect & $\begin{array}{l}\text { Core affect attributed to an Object. This process, that is isolated } \\
\text { from any judgment of the reality of the Object, is typically quick } \\
\text { and automatic but can be deliberate. }\end{array}$ \\
\hline Affect regulation & $\begin{array}{l}\text { Action aimed directly at altering core affect. This process does } \\
\text { not rely on the Object. }\end{array}$ \\
\hline Object & $\begin{array}{l}\text { The person, condition, thing, or event at which a mental state is } \\
\text { directed. An Object is a psychological representation, and } \\
\text { therefore mental states can be directed at fictions, the future, and } \\
\text { other forms of virtual reality. }\end{array}$ \\
\hline
\end{tabular}

Table 1. The main features of "core affect" model (adapted by Russell, 2003).

The "core affect" can be experienced as free floating (mood) or attributed to some cause (and thereby begins an emotional episode). In this view, an emotional response is the attribution of a change in the core affect given to a specific object (affective quality).

The main hypotheses behind this study are: i) the VR experience is able to modify the core affect; ii) the final emotional response produced by this change is necessarily influenced by the attribution process. In particular, we expect that the attribution process, being related to the previous knowledge of the users, will be different in different cultures.

To test these hypotheses we investigated the differences in the core affects and emotional episodes produced by the immersion in a distracting virtual world. To verify the role played by culture we selected two samples of ambulatory patients. Both are composed by Mexican subjects, but they differ for the cultural background: the first sample includes subjects living in El Tepeyac, a small rural and isolated Mexican village characterized by a very primitive culture; the second sample is composed by highly civilized inhabitants of Mexico City.

El Tepeyac is located at 2220 meters over the sea level, 12 hours away by car from Mexico City. Its inhabitants belong to the Tlapanecos community, a big and very poor community of Mexican indigenous who live on the mountains located at north-east of Acapulco ${ }^{33,34}$. In El Tepeyac there are 
five families, for a total of 200 persons. They live in a very marginalized condition without any kind of integration with the rest of the civilized world, and speak only a local dialect called Tlapaneco. More, they have present reliance upon subsistence-based production (based on pastoral, horticultural and/or hunting and gathering techniques), and a predominantly non-urbanized society. Only small and uncomfortable roads connect this region to the others Mexican areas. A very basic level of education (primary school) is provided to the children. Even in the school the official language is Tlapaneco dialect and education is only oral. Their most advanced technological equipments are two-way radio and closed-circuit television transmitting only local information between villages, but no news about the rest of the world. Regarding health services, few years ago they built themselves a small clinic, in which a local nurse delivers basic medications. Medical services are provided only occasionally by a voluntary medical doctor (J. L. M.) coming from Mexico City, who performs medical procedures and ambulatory surgical operations.

\section{Materials and Methods}

Subjects. Eight patients from El Tepeyac (ET), 5 females and 3 males, aged between 33 and 77 years (mean age: $50.75 \pm 16.30$ ), were involved in the study. All patients underwent an ambulatory surgical operation under local anaesthesia to remove a lipoma (3 cases) or a cyst (5 cases). Operations were performed in a small operating room in El Tepeyac, fully equipped with all medical materials needed for surgery.

The recorded physiological and psychological measures were compared with those obtained in 12 patients living in Mexico City (MC), 7 females and 5 males, aged between 19 and 74 (mean age: $49.08 \pm 16.57$ ) who were operated to remove a lipoma (5) or a cyst (7) by the same surgeon at the General and Regional Hospital No. 25 of the IMSS in Mexico City (see Tab. 2 for details).

All the operations were ambulatory interventions performed under local anaesthesia and lasted about 90 minutes. From the beginning to the end of the surgical procedure, patients were asked to wear a Head Mounted Display (HMD) and the headphones that allowed them to be fully immersed in a relaxing virtual environment ${ }^{35}$.

To participate in the study, all patients were asked to sign an informed consent. 
Technical equipment. The Playstation Portable (PSP), a Sony handheld game console that measures approximately $17 \times 7.3 \times 2.2 \mathrm{~cm}(6.7 \times 2.9 \times$ $0.9 \mathrm{in}$ ), and weighs 280 grams (9.88 ounces) was used to run the noninteractive VR environment (see below). The PSP was connected to the HMD Vuzix iWear AV 920, a high resolution head mounted display, in order to allow patients a 3D immersion in the virtual environment. The iWear weighs only 2.9 ounces, pivots up to 15 degrees for comfortable viewing angle and has integrated speakers that allow user to plug in his/her own headsets.

The relaxing environment. The Green Valley ${ }^{36}$ a non-interactive, relaxing environment showing a mountain landscape around a calm lake was presented to the patient together with a relaxing music and soft sounds (birds' songs, the water flowing, etc) (Fig. 1). Having the impression of walking around the lake, patients could observe the nature and virtually seat on a comfortable deck chair, in order to become easily relaxed.

All patients were exposed to the virtual environment for the entire length of the operation.

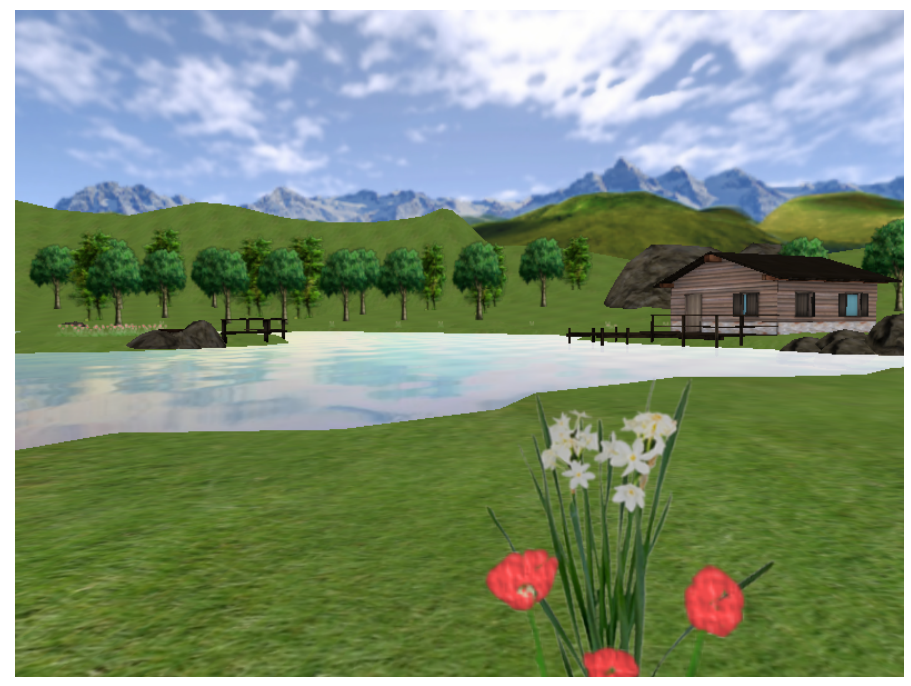

Fig. 1. Relaxing environment of the Green Valley.

Experimental procedure. All patients were asked to wear the HMD and the headphones connected to the PSP few minutes before the anaesthetic injection. The total length of the virtual relaxing session lasted about 90 
minutes, corresponding to the duration of the intervention.

Subjective measure of anxiety. Patients gave their ratings using the visual analogue scale for anxiety (VAS-A). The VAS-A is a $100-\mathrm{mm}$ vertical line with end points anchored as "no anxiety" at the bottom of the scale and "anxiety as bad as it could possibly be" at the top. The VAS anxiety scores range from 0 to 10 . The VAS-A is a widely used anxiety measurement with well-established validity and reliability ${ }^{37-41}$. Among the numerous tools available for assessing anxiety, direct scaling procedures, such as the VAS, are popular because of their simplicity, versatility, relative insensitivity to bias effects, and the assumption that the procedures yield numerical values that are valid, reliable, and on a ratio scale ${ }^{39}$. All these attributes make the VAS a very good instrument to measure discomfort even in very basic educated individuals. Ratings were administered immediately before (T0), in the middle (T1) and immediately after the operation (T2). Measurement at $\mathrm{T} 1$ was taken during a brief (approximately 2 minutes) pause in operation.

Physiological measurements. Heart rate, that is considered one of the best physiological indexes of stress, was recorded for each patient at T0, T1 and T2.

Questionnaire. After T2, only patients from El Tepeyac were asked to orally answer to the questions reported in the Tab. 3 .

\section{Results}

Data were entered into Microsoft ${ }^{\circledR}$ Excel and analyzed using $\operatorname{SPSS} \AA$, version 16. Due to the reduced number of subjects of the sample, we analyzed the data using non parametric statistic tests.

Two-tailed $p$ values of less than 0.05 were considered to be significant. Age and gender of the two groups were statistically comparable (see Tab. 2 for details). 
Emotional response to VR across different cultures

\begin{tabular}{l|lrrrrrrr}
\hline Group & Gender & Age & VAS_T0 & VAS_T1 & VAS_T2 & HR_T0 & HR_T1 & HR_T2 \\
\hline ET & male & 46 & 5 & 2 & 3 & 90 & 92 & 84 \\
ET & male & 45 & 10 & 10 & 8 & 70 & 72 & 67 \\
ET & female & 42 & 1 & 2 & 3 & 75 & 76 & 75 \\
ET & female & 73 & 1 & 1 & 1 & 78 & 78 & 75 \\
ET & female & 36 & 1 & 1 & 1 & 82 & 80 & 60 \\
ET & female & 54 & 1 & 1 & 1 & 78 & 80 & 60 \\
ET & male & 77 & 1 & 1 & 1 & 60 & 62 & 60 \\
ET & female & 33 & 1 & 4 & 1 & 100 & 85 & 85 \\
\hline Mean & & 50,75 & 2,63 & 2,75 & 2,38 & 79,13 & 78,13 & 70,75 \\
SD & & 16,30 & 3,29 & 3,11 & 2,45 & 12,14 & 8,85 & 10,53 \\
\hline & & & & & & & & \\
MC & male & 45 & 9 & 7 & 2 & 88 & 85 & 80 \\
MC & male & 53 & 6 & 3 & 1 & 64 & 67 & 72 \\
MC & male & 43 & 4 & 7 & 1 & 80 & 69 & 68 \\
MC & female & 44 & 5 & 3 & 2 & 66 & 93 & 70 \\
MC & male & 74 & 6 & 4 & 1 & 72 & 70 & 70 \\
MC & female & 19 & 5 & 1 & 1 & 95 & 95 & 93 \\
MC & male & 33 & 1 & 1 & 1 & 60 & 65 & 61 \\
MC & female & 66 & 7 & 2 & 1 & 75 & 77 & 75 \\
MC & female & 65 & 5 & 1 & 1 & 88 & 80 & 80 \\
MC & female & 68 & 4 & 1 & 1 & 72 & 87 & 85 \\
MC & female & 34 & 7 & 1 & 1 & 66 & 74 & 67 \\
MC & female & 45 & 8 & 2 & 1 & 77 & 75 & 76 \\
\hline Mean & & 49,08 & 5,58 & 2,75 & 1,17 & 75,25 & 78,08 & 74,75 \\
SD & & 16,57 & 2,11 & 2,22 & 0,39 & 10,84 & 10,04 & 8,75 \\
\hline
\end{tabular}

Table 2. This table shows demographic data (gender and age) as well as the VAS and the HR values (including means and SD) of each one of the participants (ET and MC) in the different moments (T0, T1 and T2).

Subjective measure - VAS-A scores

ET group: regarding the VAS-A scores there were no significant differences between $\mathrm{T} 1$ and $\mathrm{T} 0(\mathrm{z}=-0.272, \mathrm{~N}-$ Ties $=3, \mathrm{p}=0.785), \mathrm{T} 2$ and $\mathrm{T} 1(\mathrm{z}=-$ $0.736, \mathrm{~N}-$ Ties $=4, \mathrm{p}=0.461)$ and $\mathrm{T} 2$ and $\mathrm{T} 0(\mathrm{z}=-0.577, \mathrm{~N}-$ Ties $=3, \mathrm{p}=$ $0.564)$.

MC group: differently from what we found in the ET group, in the MC group we observed significant differences between T1 and T0 $(\mathrm{z}=-2.502, \mathrm{~N}$ - Ties $=11, \mathrm{p}=0.012), \mathrm{T} 2$ and $\mathrm{T} 1(\mathrm{z}=-2.384, \mathrm{~N}-$ Ties $=7, \mathrm{p}=0.017)$ and $\mathrm{T} 2$ and $\mathrm{T} 0(\mathrm{z}=-2.946, \mathrm{~N}-$ Ties $=11, \mathrm{p}=0.003)$.

Comparing the difference between the VAS-A values calculated from the beginning to the end of the operation (T0 - T2) we found a significant difference between the two groups of patients $\left(\mathrm{U}=4.500, \mathrm{~N}_{1}=8, \mathrm{~N}_{2}=12, \mathrm{p}\right.$ $=0.0001)$ indicating that the difference in the VAS-A scores between T0 
and $\mathrm{T} 2$ was greater in the MC group than in the ET group.

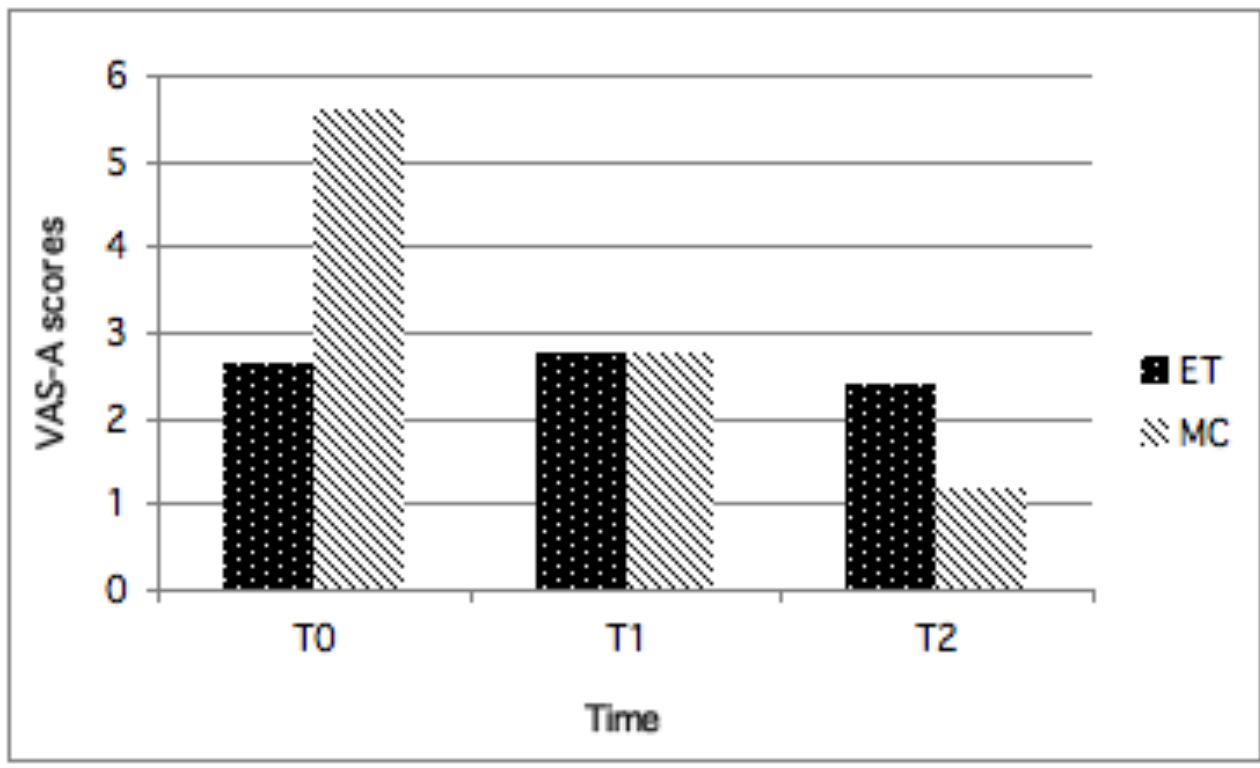

Fig. 2. VAS-A scores in the two groups of patients from the beginning (T0) to the end of the operation (T2).

Physiological measure - heart rate values.

ET group: regarding the HR there were significant differences between T2 and $\mathrm{T} 1(\mathrm{z}=-2.371, \mathrm{~N}-\mathrm{Ties}=7, \mathrm{p}=0.018)$ and between $\mathrm{T} 2$ and $\mathrm{T} 0(\mathrm{z}=-$ 2.207, $\mathrm{N}-$ Ties $=6, \mathrm{p}=0.027)$, but not between T1 and T0 $(\mathrm{z}=-0.526, \mathrm{~N}-$ Ties $=7, \mathrm{p}=0.599$ ).

MC group: differently from what we found in the ET group, in the MC group we observed no significant differences in HR between $\mathrm{T} 1$ and $\mathrm{T} 0$ ( $\mathrm{z}=$ $-0.713, \mathrm{~N}-$ Ties $=11, \mathrm{p}=0.476), \mathrm{T} 2$ and $\mathrm{T} 1(\mathrm{z}=-1.893, \mathrm{~N}-$ Ties $=10, \mathrm{p}=$ $0.06)$ and $\mathrm{T} 2$ and $\mathrm{T} 0(\mathrm{z}=-0.357, \mathrm{~N}-$ Ties $=11, \mathrm{p}=0.721)$.

Comparing the difference between the HR values calculated from the beginning to the end of the operation ( $\mathrm{T} 0-\mathrm{T} 2)$ we found a significant difference between the two groups of patients $\left(\mathrm{U}=30.000, \mathrm{~N}_{1}=8, \mathrm{~N}_{2}=12\right.$, $\mathrm{p}=0.049$ ) indicating that the difference in the HR values between T0 and $\mathrm{T} 2$ was greater in the ET group than in the MC group. 


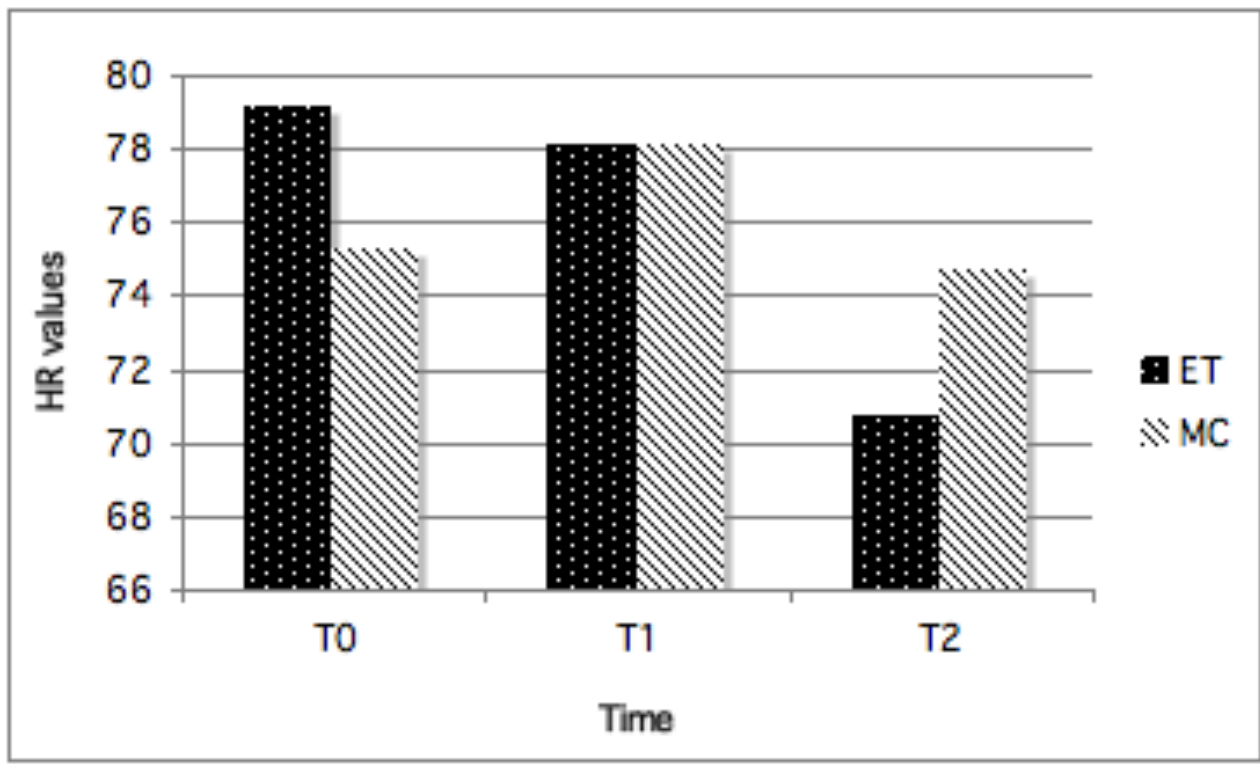

Fig. 3. VAS-A scores in the two groups of patients from the beginning (T0) to the end of the operation (T2).

Questionnaire. Because one of the goals of the study was to investigate the effect of VR on people without any previous experiences with technology, we proposed the questionnaire presented in the Tab. 3 only to the ET group. Questions and answers were given orally.

What we noticed from their answers is that: seven out of eight experienced closed-circuit television before; none of them played with videogames before, and nobody have ever heard about VR. Despite this, only one declared to be worried about technology. One patient was so absorbed by VR that he said he did not realize that he was undergoing an operation and the majority of them said that they did not feel pain and anxiety during the operation. All patients answered that they were interested in the virtual landscape, that they would like to repeat the experience with VR and that they would recommend the VR experience to some else. They did not passively observed the virtual environment as suggested by the fact that they also suggested how to modify it: some of them expressed the desire to see animals moving around, one said she would like to see a seaside landscape populated by fishes and dolphins and another one would to include landscape of the region in which they live as well as pictures of his family. In particular, the answers to the question number 10 demonstrate that after the experience, they have clearly understood the VR concept: a virtual 
reality environment is "a place" that can be modified on the basis of the user's needs and tastes. This is exactly the same desire that in the last decades has moved technological societies to the creation of "virtuality". 


\section{Questions}

\section{Patients' Answers}

\begin{tabular}{|c|c|c|c|c|}
\hline 1 & $\begin{array}{l}\text { Do you use to watch } \\
\text { television? }\end{array}$ & Yes (6) & Less than 2 hours per week (1) & No (1) \\
\hline 2 & $\begin{array}{l}\text { Have you ever played with } \\
\text { videogames before? }\end{array}$ & Yes & & No $(8)$ \\
\hline 3 & $\begin{array}{l}\text { Have you heard about virtual } \\
\text { reality before this experience? }\end{array}$ & Yes & & No $(8)$ \\
\hline 4 & $\begin{array}{l}\text { Are you worried/scared about } \\
\text { technology? }\end{array}$ & Yes (1) & A little & No (7) \\
\hline 5 & $\begin{array}{l}\text { Wearing the HMD, did you } \\
\text { realize you were undergoing } \\
\text { an operation? }\end{array}$ & Yes (7) & A little & No (1) \\
\hline 6 & $\begin{array}{l}\text { Did you feel pain during the } \\
\text { operation? }\end{array}$ & Yes & A little (1) & No (7) \\
\hline 7 & $\begin{array}{l}\text { Did you feel anxious during } \\
\text { the operation? }\end{array}$ & Yes & A little (2) & No (6) \\
\hline 8 & $\begin{array}{l}\text { Were you interested in the } \\
\text { virtual landscape we showed } \\
\text { you? }\end{array}$ & Yes (8) & A little & No \\
\hline 9 & $\begin{array}{l}\text { While there, did you fell } \\
\text { asleep? }\end{array}$ & Yes (1) & A little & No (7) \\
\hline 10 & $\begin{array}{l}\text { Would you propose a different } \\
\text { type of virtual environment? }\end{array}$ & Yes (8) & Don't know & No \\
\hline 11 & $\begin{array}{l}\text { Would you repeat the virtual } \\
\text { experience if necessary? }\end{array}$ & Yes (8) & Don’t know & No \\
\hline 12 & $\begin{array}{l}\text { Were you relaxed during the } \\
\text { immersion in the virtual } \\
\text { environment? }\end{array}$ & Yes (8) & A little & No \\
\hline 13 & $\begin{array}{l}\text { Are you going to recommend } \\
\text { VR to other patients? }\end{array}$ & Yes (8) & A little & No \\
\hline 14 & $\begin{array}{l}\text { Do you think VR is a useful } \\
\text { tool to reduce anxiety and } \\
\text { discomfort during a surgical } \\
\text { operation? }\end{array}$ & Yes (8) & A little & No \\
\hline
\end{tabular}

Tab. 3. Oral questionnaire administered to El Tepeyac participants. 


\section{Discussion}

Our data show that, from the beginning to the end of the operation, there was a significant decrease in the level of perceived anxiety in the MC group but not in the ET group and, vice versa, a significant decrease in HR in the ET group, but not in the MC group. The decrease in the VAS-A and HR was significantly different in the two groups indicating that the VR exposure had a significant relaxing effect on both groups, but in one case (MC) the effect caused a reduction in the perceived anxiety, while in the other (ET) it resulted in a decrease of HR, that is the arousal component of the core affect theory. A possible explanation of these results is that an emotion directed to an object (VR in this case) is not a primitive element, but a complex event characterized by different components ${ }^{42}$ such as autonomic and psychological factors. According to the Russell's theory, core affect automatically responds to the contents of consciousness whether based on reality or fiction. So, our relaxing environment activated a relaxation state in all the patients, regardless their cultural background or their confidence with technology. On the contrary, culture and subjects' previous experiences altered their attribution process. Specifically, the lack of a significant reduction in the VAS-A found in the ET group does not necessarily mean they did not benefit from the VR relaxing experience, but that they were not able to attribute the modifications in their physiological response (core affect) to it. In this view, these data are an empirical demonstration that the traditional sharp boundary between emotions and non-emotions can be changed in favor of a model in which each of the components that concurs in creating a complex emotion can also occur alone.

Nevertheless, because at the level of perceived anxiety experienced by people from El Tepeyac was relatively low even at the beginning of the operation, other two alternative cultural-related explanations to the lack of reduction in the VAS-A score in this group of patients are possible. First: the total lack of medical knowledge that characterizes people from El Tepeyac could be a factor that significantly limits their worries about the possible negative consequences of the operation and the illness itself. Second: we asked them to report their level of anxiety on the VAS trying to explain them what anxiety is. Again, due to their lack of medical information, it is possible that they incorrectly interpreted what we were referring to. If this was the case, the VAS did not really measured what we intended to evaluate. All these explanations are possible and congruent with 
the very different cultural background that characterizes the two samples of patients.

The main limitation of this study is that the sample is small: as clearly stated in the introduction, El Tepeyac is a very isolated place with no stable medical services. Unfortunately, testing a larger number of patients would be a matter of years. So we started to collect and analyze data from a small sample of subjects assuming that they were representative of the entire population, with the intention to collect more data in the future. The consequence of such a small number of subjects is the lack of a control experimental condition, such as the lack of treatment or the administration of a different relaxation technique not based on virtual reality. Moreover, the strength of this study is that it represents the first attempt to analyze the effects of VR exposure in two samples of patients characterized by a huge different cultural and technological background: the few available crosscultural studies on VR investigated how the user's character and his/her cultural background influence the sense of presence ${ }^{43,44}$ in two different cultures (i.e. Dutch and Chinese), but very similar regarding the use of interactive media and technology.

In conclusion, this study shows that the VR exposure to a relaxing environment have different physiological and psychological effects according to the cultural and technological background of the users. In particular, we interpret the different emotional responses between the two groups with a difference in the subjects' attribution process mainly caused by their different cultural background. Further researches are needed to investigate the effects of other, and possibly more interactive VR systems in such kind of rural isolated populations. 


\section{References}

1. Lang PJ. The emotion probe: Studies of motivation and attention. American Psychologist. 1995;50(5):372-85.

2. Lang PJ. Negative video as structure: Emotion, attention, capacity and memory. Journal of Broadcasting and Electronic Media. 1996;40(4):460-77.

3. Lazarus RS, Speisman JC, Mordkoff AM, Davison LA. A laboratory study of psychological stress produced by a motion picture film. Psychological Monographs. 1962;76(553):1-122.

4. Gross JJ, Levenson RW. Emotion elicitation using films. Cognition \& Emotion. 1995;9(1):87-108.

5. Riva G, Mantovani F, Capideville CS, et al. Affective interactions using virtual reality: the link between presence and emotions. Cyberpsychology and Behavior. 2007 Feb;10(1):45-56.

6. Botella C, Garcİa-Palacios A, Villa H, et al. Virtual reality exposure in the treatment of panic disorder and agoraphobia: A controlled study. Clinical Psychology \& Psychotherapy. 2007;14(3):164-75.

7. Han $\mathrm{K}, \mathrm{Ku}$ J, Kim K, et al. Virtual reality prototype for measurement of expression characteristics in emotional situations. Computers in biology and medicine. 2009 Feb;39(2):173-9.

8. Vincelli F. From imagination to virtual reality: the future of clinical psychology. CyberPsychology \& Behavior. 1999;2(3):241-8.

9. Gorini A, Riva G. Virtual reality in anxiety disorders: the past and the future. Expert Review of Neurotherapeutics. 2008;8(2):215-33.

10. Riva G. Virtual reality in psychotherapy: review. CyberPsychology \& Behavior. 2005 Jun;8(3):220-30; discussion 31-40.

11. Levandovski R, Ferreira MB, Hidalgo MP, Konrath CA, da Silva DL, Caumo W. Impact of preoperative anxiolytic on surgical site infection in patients undergoing abdominal hysterectomy. Am J Infect Control. 2008 Dec;36(10):718-26.

12. Muglali M, Komerik N. Factors related to patients' anxiety before and after oral surgery. J Oral Maxillofac Surg. 2008 May;66(5):8707.

13. Rubin GJ, Hardy R, Hotopf M. A systematic review and metaanalysis of the incidence and severity of postoperative fatigue. $\mathrm{J}$ Psychosom Res. 2004 Sep;57(3):317-26. 
14. Gold JI, Belmont KA, Thomas DA. The neurobiology of virtual reality pain attenuation. Cyberpsychol Behav. 2007 Aug;10(4):53644.

15. Sharar SR, Carrougher GJ, Nakamura D, Hoffman HG, Blough DK, Patterson DR. Factors influencing the efficacy of virtual reality distraction analgesia during postburn physical therapy: preliminary results from 3 ongoing studies. Arch Phys Med Rehabil. 2007 Dec;88(12 Suppl 2):S43-9.

16. Sharar SR, Miller W, Teeley A, et al. Applications of virtual reality for pain management in burn-injured patients. Expert Rev Neurother. 2008 Nov;8(11):1667-74.

17. Hoffman HG. Virtual-reality therapy. Sci Am. 2004 Aug;291(2):5865 .

18. Hoffman HG, Doctor JN, Patterson DR, Carrougher GJ, Furness TA, 3rd. Virtual reality as an adjunctive pain control during burn wound care in adolescent patients. Pain. 2000 Mar;85(1-2):305-9.

19. Hoffman HG, Patterson DR, Seibel E, Soltani M, Jewett-Leahy L, Sharar SR. Virtual reality pain control during burn wound debridement in the hydrotank. Clin J Pain. 2008 May;24(4):299-304.

20. Hoffman HG, Patterson DR, Soltani M, Teeley A, Miller W, Sharar SR. Virtual Reality Pain Control during Physical Therapy Range of Motion Exercises for a Patient with Multiple Blunt Force Trauma Injuries. Cyberpsychol Behav. 2008 Nov 19.

21. Hoffman HG, Richards TL, Bills AR, et al. Using FMRI to study the neural correlates of virtual reality analgesia. CNS Spectr. 2006 Jan;11(1):45-51.

22. Hoffman HG, Richards TL, Van Oostrom T, et al. The analgesic effects of opioids and immersive virtual reality distraction: evidence from subjective and functional brain imaging assessments. Anesth Analg. 2007 Dec;105(6):1776-83, table of contents.

23. Hoffman HG, Seibel EJ, Richards TL, Furness TA, Patterson DR, Sharar SR. Virtual reality helmet display quality influences the magnitude of virtual reality analgesia. J Pain. 2006 Nov;7(11):84350.

24. Hoffman HG, Sharar SR, Coda B, et al. Manipulating presence influences the magnitude of virtual reality analgesia. Pain. 2004 Sep;111(1-2):162-8.

25. Schneider SM, Prince-Paul M, Allen MJ, Silverman P, Talaba D. Virtual reality as a distraction intervention for women receiving chemotherapy. Oncol Nurs Forum. 2004 Jan-Feb;31(1):81-8. 
26. Gold JI, Kim SH, Kant AJ, Joseph MH, Rizzo AS. Effectiveness of virtual reality for pediatric pain distraction during i.v. placement. Cyberpsychol Behav. 2006 Apr;9(2):207-12.

27. Freeman D. Creating emotion in games. The craft and art of emotioneering. Indianapolis, IN: New Riders; 2003.

28. Fogg BJ. Persuasive technology: Using computers to change what we think and do. San Francisco, CA: Morgan Kaufmann Publishers; 2003.

29. Botella C, Quero S, Banos RM, Perpina C, Garcia Palacios A, Riva G. Virtual reality and psychotherapy. Stud Health Technol Inform. 2004;99:37-54.

30. Riva G, Molinari E, Vincelli F. Interaction and presence in the clinical relationship: virtual reality (VR) as communicative medium between patient and therapist. IEEE Transactions on Information Technology in Biomedicine. 2002 Sep;6(3):198-205.

31. Riva G, Waterworth JA, Waterworth EL. The Layers of Presence: a bio-cultural approach to understanding presence in natural and mediated environments. Cyberpsychology \& Behavior. 2004;7(4):405-19.

32. Russell JA. Core Affect and the Psychological Construction of Emotion. Psychological Review. 2003;110(1):145-72.

33. Instituto Nacional de estadistica GeI.

$<\mathrm{http}$ ://www.google.com.mx/search?hl=es\&q=inegi $+\mathrm{e}+$ indigenas $+\mathrm{tl}$ apanecos\&btnG=Buscar\&meta $>$. Accessed.

34. Instituto Nacional de estadistica GeI.

35. Riva G, Gaggioli A, Villani D, et al. NeuroVR: an open source virtual reality platform for clinical psychology and behavioral neurosciences. Stud Health Technol Inform. 2007;125:394-9.

36. Grassi A, Gaggioli A, Riva G. The green valley: the use of mobile narratives for reducing stress in commuters. Cyberpsychol Behav. 2009 Apr;12(2):155-61.

37. Abu-Saad H, Holzemer WL. Measuring children's self-assessment of pain. Issues Compr Pediatr Nurs. 1981 Sep-Dec;5(5-6):337-49.

38. Downie WW, Leatham PA, Rhind VM, Wright V, Branco JA, Anderson JA. Studies with pain rating scales. Annals of the Rheumatic Diseases. 1978;37:378-81.

39. Price DD, McGrath PA, Rafii A, Buckingham B. The validation of visual analogue scales as ratio scale measures for chronic and experimental pain. Pain. 1983 Sep;17(1):45-56. 
40. Reading AE. A comparison of pain rating scales. J Psychosom Res. 1980;24(3-4):119-24.

41. Vessey JA, Carlson KL, McGill J. Use of distraction with children during an acute pain experience. Nurs Res. 1994 NovDec;43(6):369-72.

42. Oatley K, Johnson-Laird PN. Towards a cognitive theory of emotions. Cognition and Emotion. 1987;1:29-50.

43. Chang T, Wang X, Lim KJ. Cross-cultural communication, media and learning processes in asynchronous learning networks. 35th Annual Hawaii International Conference on System Sciences. Maui, Hawaii, 2002:113-22.

44. $\mathrm{Hu}$ J, Bartneck C. Culture matters: a study on Presence in an interactive movie. Cyberpsychology and behaviour 2008;11(5):52937. 



\section{CHAPTER VIII}

\section{A Second Life for eHealth: prospects for the use of $3 \mathrm{D}$ virtual worlds in clinical psychology}

A. Gorini, A. Gaggioli, C. Vigna, G. Riva.

Journal of Medical Internet Research. 2008, Aug 5;10(3):e21. 



\section{Abstract}

The aim of the present paper is to describe the role played by threedimensional (3-D) virtual worlds in eHealth applications, addressing some potential advantages and issues related to the use of this emerging medium in clinical practice. Due to the enormous diffusion of the World Wide Web (WWW), telepsychology, and telehealth in general, have become accepted and validated methods for the treatment of many different health care concerns. The introduction of the Web 2.0 has facilitated the development of new forms of collaborative interaction between multiple users based on 3-D virtual worlds. This paper describes the development and implementation of a form of tailored immersive e-therapy called phealth whose key factor is interreality, that is, the creation of a hybrid augmented experience merging physical and virtual worlds. We suggest that compared with conventional telehealth applications such as emails, chat, and videoconferences, the interaction between real and 3-D virtual worlds may convey greater feelings of presence, facilitate the clinical communication process, positively influence group processes and cohesiveness in groupbased therapies, and foster higher levels of interpersonal trust between therapists and patients. However, challenges related to the potentially addictive nature of such virtual worlds and questions related to privacy and personal safety will also be discussed. 


\section{Introduction}

Since the introduction of the Web 2.0 in 2004 [1], there has been a huge increase in the potential of Web applications, allowing users to create, modify, and share contents using multiple computers in various locations. The Web 2.0 is a read-write Web that facilitates social networking, collaboration, and participation between users [2,3]. One hugely successful application of the Web 2.0 is represented by three-dimensional (3-D) virtual worlds (e.g., Second Life [4], There [5], and Active Worlds [6]). These computer-based, simulated environments are characterized by the simultaneous presence of multiple users who inhabit and interact via avatars within the same simulated space. The computer-simulated world typically appears similar to the real world, with real world rules such as gravity, topography, locomotion, real-time actions, and communication. Over the last few years, the number of virtual world users has increased dramatically, and today, Second Life, the largest 3-D online digital world, boasts some 12 million subscribers. 3-D virtual worlds can be considered as 3-D social networks, where people can collaborate to create and edit objects (like a collaborative 3-D wiki space) besides meeting each other and interacting with existing objects. Compared with the conventional Web 1.0 applications, virtual worlds offer novel ways to develop social skills; socialize and interact with other people via customizable, realistic, 3-D, fully textured, and animated avatars; attend and participate in live events like lectures and conferences; build communities, including learners' communities and patient support groups; relax and visit new places; and browse document collections in 3-D virtual libraries. 3-D virtual reality (VR) worlds also show great potential for health purposes. In particular, Second Life currently features a number of medical and health education projects. By way of example, the Nutrition Game proposed by the Ohio University $[7,8]$ simulates choices a user can make in various restaurants and informs the player about the health impacts of those choices. The Heart Murmur Sim $[9,10]$ provides an educational virtual world for cardiac auscultation training that enables clinical students to tour a virtual clinic and test their skills at identifying the sounds of different types of heart murmurs. The Second Life Virtual Hallucinations Lab $[11,12]$ aims to educate people about schizophrenic hallucinations. The Gene Pool [13] is an interactive genetics lab and learning area featuring simulated lab experiments, tutorials, and simple videos to enhance the learning experience. The Virtual Neurological Education Centre (VNEC) [14] demonstrates a virtual 
simulated online experience where people are able to actively expose themselves to the most common symptoms that a person suffering from a neurological disability may encounter, and the HealthInfo Island [15] is funded by the US National Library of Medicine (NLM) to provide consumer health information services. All of these virtual initiatives are mainly centered on the promotion of an innovative form of public health consisting of the diffusion of medical information and the education of therapists and patients [16].

The aim of the present paper is to introduce and discuss the use of 3-D virtual worlds for an innovative online health service called p-health. Phealth provides personalized immersive e-therapy whose key factor is interreality [17], ie the creation of a hybrid-augmented experience merging the physical and virtual world. In p-health, the interreality experience is achieved through the following:

- an extended sense of presence [18-20]: P-health uses advanced simulations (3-D virtual worlds) to transform health guidelines and provisions into experience. In p-health, users do not receive abstract information but live meaningful experiences.

- an extended sense of community (social presence): P-health uses hybrid social interaction and dynamics of group sessions to provide each user with targeted-but also anonymous, if required-social support in both the physical and virtual world.

- real-time feedback between the physical and virtual worlds: P-health uses bio and activity sensors and devices (eg, PDAs, mobile phones) to track both the behavior and the health status of the user in real time and to provide targeted suggestions and guidelines. The feedback activity is twofold: (1) behavior in physical world influences the experience in the virtual one (eg, if I eat too much and I do not exercise, my avatar will become fatter), and (2) behavior in the virtual world influences the experience in the real one (eg, if I participate in the virtual support group, I can exchange SMS messages with the other participants during the day).

Our hypothesis is that the introduction of the p-health approach in eHealth services could extend the potential of the Web 2.0 and shared 3-D worlds to therapists and patients. To support this claim, the paper will describe how the use of avatars can improve social presence. Further, we will focus on the existing applications of 3-D worlds in clinical settings and address some ethical considerations and possible pitfalls of using 3-D worlds for therapeutic purposes. Finally, we will introduce a possible p-health scenario we are developing in Second Life for the treatment of addiction disorders. 


\section{Psychological features in avatar-based interaction}

The p-health approach suggests that providing remote patients with a feeling of social presence [21] plays a crucial role in improving therapeutic effectiveness. Through social presence, users experience a feeling of inhabiting a shared space with one or more others, and their awareness of mediation by technology recedes into the background [22]. Social presence required participants to experience themselves as co-located and mutually aware of, responsive to, and responsible to one another [23]. As suggested by Casanueva and Blake [24], the sense of social presence consists of the belief that the other people in the virtual environment are real and really present and that the user and the others are part of a group and process.

We suggest that 3-D virtual worlds are able to convey strong feelings of social presence through avatar interaction, enhancing the feeling of togetherness of remote users who are connected through some form of telecommunication medium. Results of recent studies about avatar-based social interaction lend support to this hypothesis [25]. In their research, Bente and colleagues [26] measured social presence and interpersonal trust in avatar-based collaborative net communications, comparing this condition with face-to-face communication as well as with audio-based (phone) and text-based Web communication. The results from 48 participants showed that the level of co-presence was higher in avatar-based interactions than in phone or chat interactions. In a subsequent study, Bente and colleagues [27] investigated the experience of social presence as a relevant effect dimension of avatar-mediated Web communication. A total of 142 participants were randomly assigned to one of five possible communication settings: (1) text only, (2) audio only, (3) audio and video, (4) audio and low fidelity avatar, (5) audio and high fidelity avatar. Results revealed a significant difference between text and all other communication modes, indicating that audio, video, and avatar systems work similarly and better than text alone in creating the experience of social presence. However, according to the authors, avatar platforms offer new potentials to overcome many of the restrictions related to audio and video communication modes. In particular, they suggest that virtual worlds and avatars play a critical role in contextualizing social interaction and fostering the salience of nonverbal information by providing active filtering and contingency management systems as opposed to being just the virtual equivalents of a video conferencing system.

Other studies have suggested that even avatars with rather primitive 
expressive abilities may elicit strong emotional responses in users sharing a collaborative virtual environment. Experiments have shown that the avatar can readily take on a personal role, increasing the sense of community feeling and becoming a genuine representation of the underlying individual, not only visually, but also within a social context [28]. Moreover, Yee and colleagues [29] investigated whether norms about social space in the real world map onto how avatars act in relation to each other in virtual space. In an observational study, the authors collected data from avatars in order to explore whether social norms of gender, interpersonal distance (IPD), and eye gaze transfer into virtual environments even though the modality of movement is entirely different. They found that, as in the real world, malemale dyads tend to stand further apart and look at each other much less than female-female dyads: (1) male-male dyads have larger IPDs than femalefemale dyads, (2) male-male dyads maintain less eye contact than femalefemale dyads, and (3) decreases in IPD are compensated with gaze avoidance. In summary, all these preliminary findings suggest that avatarbased interaction in virtual worlds may have the potential to enrich the level of emotional connections and social presence conveyed by conventional telehealth tools, such as Internet, videoconferencing, email, and telephone.

From a clinical perspective, the advantages presented by the $3-\mathrm{D}$, avatarbased interactions serve to facilitate the communication process between therapists and patients, to positively influence group cohesiveness in groupbased therapies and to create greater levels of interpersonal trust, which is a fundamental requirement to establish a successful therapeutic alliance.

\section{3-D virtual worlds as a support tool for psychological interventions}

The strong sense of presence and social connection elicited by avatar interaction suggests two possible clinical applications of 3-D virtual worlds. The first regards the potential to provide VR-based treatments within the online virtual worlds, and the second regards the creation of online virtual communities of patients.

\section{3-D Virtual Worlds and Virtual Reality Exposure Therapy}

In recent years, a number of studies have suggested the efficacy of VR exposure therapy in the diagnosis and treatment of various psychological disorders. Positive results have been obtained in the treatment of specific 
phobias (in particular, aviophobia, acrophobia, fear of driving, claustrophobia, and arachnophobia), eating disorders, social anxiety disorders, sexual disorders, posttraumatic stress disorder, and panic disorder with or without agoraphobia [30,31].

During the VR exposure, the patient is immersed in a virtual environment containing the critical stimulus. This procedure, which has been shown to be at least as effective as traditional techniques in reducing phobic symptoms [32], presents some practical advantages offered by the use of VR technology. As stimuli are computer generated, the therapist has full control over their intensity, and the risk of unpredictable effects is significantly lower than in vivo exposure since subjects have the opportunity to explore threatening aspects of reality in a safe environment where consequences are not real [33]. Further, virtual exposure provides an opportunity to present the patient with realistic 3-D visualization of the feared situation, which is more effective than imagination, especially when the patient is unable to recreate the critical scenarios because of pathological avoidance of problematic memories, as is often the case in posttraumatic stress disorder [34]. When used in combination with specific instruments, the VR exposure has the added advantage of allowing therapists to record different psychophysiological parameters before, during, and after exposure to the feared stimuli in order to obtain objective measures of the individual modifications. 3-D virtual worlds appear to have much to offer to exposure therapy of this kind. The therapist and patient share the same online virtual space and, in this way, the therapist can accompany the patient through a particular threatening experience just by logging onto a specific website and adopting a preferred avatar. Interaction can be modified on the basis of therapeutic needs. In the case of social phobia, for example, after practicing with the therapist within a closed environment (i.e., the therapist's virtual office), the patient can be taken to a virtual world populated by other avatars and asked to initiate a conversation and obtain feedback from them in real-time audio through the use of a microphone. Similarly, patients with agoraphobia can be exposed to a variety of unfamiliar worlds different from those the clinician can provide in an office setting. Patients suffering from addiction disorders (e.g., drug abuse, pathological gambling, food craving) can be exposed to specific kinds of dangerous stimuli without running the risk of "succumbing to temptation" [35].

\section{3-D Virtual Worlds for Creating Virtual Communities of Patients}

3-D virtual worlds may have the potential to bring several innovative features to virtual patient communities by providing mediated environments 
with appropriate social, nonverbal, and contextual information that previous Web applications (Web 1.0) were unable to convey. Winkelman and Choo [36] surmised that patients with chronic diseases possess a particular tacit knowledge gleaned from their personal experience of illness and experientially acquired by having to cope with the daily challenges and needs posed by a chronic disease. These needs include information on the disease, treatment side effects, treatment plans, professional contacts, as well as supportive information for family and friends. According to the authors, if this tacit knowledge can be shared or socialized through a program, tool, or medium, a patient's sense of self-efficacy can improve, thereby positively affecting health outcomes as well as social functioning. This approach argues for a shift in the role of chronic disease patients from external consumers of health care services to a community of practice of internal customers. Introduced by Wenger (1998), communities of practice are social constructs that bring learning into lived experience of participation in the world [37]. They are defined as self-organizing, informal groups, whose members work together toward common goals, face common needs, share best practices, and have a common identity. Drawing on these concepts, Winkelman and Choo [36] suggest that with the implicit support of health care organizations, patients can benefit from gaining access to the expertise of peers by integrating knowledge gained from the experiences of living with chronic disease into their self-management. In particular, they claim that virtual patient communities can become effective tools of communication if (1) members have common interests, needs, goals, as well as an aspiration for mutual communication and the furthering of relationships, and (2) they are able to supplement already existing face-toface communication opportunities. Even in this case, the possibility to share specific virtual environments from different parts of the world and to interact via customizable avatars can presumably facilitate the development and the diffusion of online communities of practice allowing an efficient exchange of medical and experiential information between patients and experts.

\section{Existing therapeutic applications in Second Life}

In this section we will briefly explore some of the Second Life virtual environments specifically created for therapeutic purposes. Inspired by the therapeutic success obtained with different kinds of virtual treatments 
$[38,39]$, and taking advantage of the potential of the Second Life platform, Brain Talk Industries, the largest nonprofit organization in the world dedicated to providing online communities for patients and caregivers dealing with neurological issues, has created Brigadoon, a private island in Second Life specifically designed for patients with Asperger's syndrome. Brigadoon aims at providing an ideal place for people with a form of highfunctioning autism, characterized by enormous difficulties in social interactions, to develop their social skills by interacting with other people dealing with the same problems [40]. After their initial experiences inside Brigadoon, many patients began venturing into Second Life proper and mixing with non-autistic people. Some of them are now active participants in other communities, including two autistic women who have formed "the autistic liberation front," a Second Life space where autistic people can organize, educate, and advocate for themselves [41-43]. A similar aim underlies the creation of Live2Give [44], a Second Life island dedicated to people affected by cerebral palsy. Like Brigadoon, this virtual place brings people together, giving them the possibility to help each other cope with their common struggles. According to Lester, the experience appears to be empowering and revolutionizes the way the users feel about themselves and the part they have to play in the world [45]. Similarly, a British organization called ARCI has developed a virtual environment in Second Life to help abused children learn important life skills. The children enter the virtual world to learn to socialize and work as a team and to learn essential computer skills [46]. A very interesting therapeutic experience related to Second Life is described by Roberto Salvatierra, a medical student suffering from agoraphobia. Within Second Life, he created an avatar that closely resembled his own real-life appearance. By seeing himself in a simulated 3$\mathrm{D}$ environment, Roberto felt he could become more comfortable with unfamiliar open spaces and this was exactly what happened. Thanks to his personal positive experience he decided to set up an in-world group called the "Agoraphobia Support Group," which he hopes other people with agoraphobia will join to discuss their shared difficulties [47,48].

These examples show how 3-D online virtual worlds can provide a richer variety of tools than email or typing text onto bulletin boards, including the opportunity to build new customized environments, create avatars, interact with others without revealing one's real identity (i.e., the real physical disabilities one has in the real world), and communicate with people in a way that more closely resembles face-to-face meetings. Moreover, the possibility to buy gestures - animations of avatars making faces - enriches the way in which users can communicate and represent themselves in these 
experiential virtual worlds. So, even if the main aim of these virtual online communities is to support rather than treat patients, their success proves the potential of 3-D virtual worlds to become very useful tools for an innovative form of eHealth dedicated to patients with mental illness $[16,49]$.

Despite the positive data we have presented, the use of the Internet to provide mental health services is controversial, and, in the ongoing debate about the value and ethics of therapeutic virtual environments, there are proponents at both extremes. Some conceive of technology as means to a bright future where anyone's emotional needs can be instantaneously addressed; others are obstinately opposed to the use of distance psychology for any kind of intervention. In our view, virtual therapy is most effective when it is used as an adjunct to traditional therapy or as part of an aftercare plan. For these reasons, we advise against any kind of therapy being practiced exclusively on the Web because of its supportive rather that exhaustive nature. This point must be made clear to online therapy providers and the general public.

\section{Ethical considerations and important caveats in the use of 3- D virtual worlds for eHealth}

Although the therapeutic potential of 3-D virtual worlds is quite promising, there are challenges associated with an approach of this kind that need to be addressed. In fact, if it is true that people can explore threatening aspects of reality in a safe environment, it also is true that if the use of online worlds becomes excessive, there is a risk that it will prevent people from forming meaningful real-world relationships. In fact, as observed by Allison et al [33], an increased substitution of cyberspace-based relationships at the expense of face-to-face interaction may create a developmental doubleedged sword. In the case of socially anxious patients, for example, the Internet is useful to modify peer group interactions, while it does little to foster the development of genuine intimacy. When exposing patients to virtual environments, therapists should consider the risk of Web addiction and encourage patients to participate in real-life social interaction as much as possible.

Another critical point regards anonymity: the chance to remain anonymous offers a less intimidating opportunity for social interaction and psychological reflection and would allow more people to discreetly seek help on their own. On the other side, anonymity represents a significant risk 
for patients and therapists. The computer-based interface does not guarantee that the person on the other side of the screen is really who we expect, and anybody can enter the virtual environment and interact with patients, producing negative effects on their experience and introducing uncontrollable and disturbances variables in the environment. These aspects can be overcome, for example, by creating private servers specific for controller environments designed and dedicated to therapy and using protection codes personally given by the therapist to the patients.

Regarding the therapists, they need to first conduct self-assessment and then enhance their knowledge and skills in using these alternative forms of therapy [50] since the provision of eHealth services is not simply a click of the mouse [51].

Besides the previous more clinical considerations, there are some very challenging issues that need to be resolved to ensure the safe and ethical use of eHealth in general. These include complex and interrelated questions of security, confidentiality, and privacy; licensure requirements; competency; standards of care; and reimbursement that must be considered by practitioners, researchers, consumers, health care organizations, managed care companies, and federal and state legislatures [52].

The American Psychological Association (APA) has published a statement entitled "Services by Telephone, Teleconferencing, and Internet" [53]. This statement stipulates that in the absence of specific telehealth standards, psychologists must take reasonable steps to ensure competence in providing services and to protect patients, clients, and research participants from harm. The APA is also developing recommendations for the board regarding ethical, legal, and clinical concerns related to the practice of telehealth, with the aim of providing practitioners with information about electronic activities. While conducting interventions via telehealth applications, patients may believe that the Internet sessions are secure and completely private and confidential. To safeguard against a breach in confidentiality, therapists and clinicians should fully inform patients of the limits of confidentiality associated with telehealth and other forms of telecommunications. In sum, the use of 3-D virtual worlds as an advanced form of eHealth holds great promise as long as their limitations and associated risks are taken into consideration as well. 


\section{The use of 3-D virtual worlds in clinical practice}

In the Introduction, we presented $\mathrm{p}$-health as a possible new paradigm for eHealth. From a technological viewpoint, a possible p-health scenario would be based on the following three technologies: 3-D virtual worlds, bio and activity sensors, and personal digital assistants (PDAs) and/or mobile phones. Each will be considered in turn below.

\section{3-D Virtual Worlds}

As we have discussed previously, 3-D virtual worlds enable their users to interact with each other through motional avatars. Residents can explore the world, meet other users, socialize, participate in individual and group activities, and buy items (virtual property) and services from one another.

Bio and Activity Sensors (Connection from the Real World to the Virtual One)

Typically 3-D virtual worlds are closed worlds and in no way reflect the real activity and status of the users. In p-health, bio and activity sensors are used to track the health status of users and to influence their experiences in the virtual world (avatar, activity, and access). The link between real and virtual worlds would be in real time, allowing the development of advanced biofeedback settings, but would also ensure health tracking even in situations where an Internet connection is unavailable.

\section{PDAs and/or Mobile Phones (Connection from the Virtual World to the} Real One)

In p-health, the social and individual user activities in the virtual world have a direct link with his or her life through a PDA and/or mobile phone. This link is at three levels:

1. follow-up: It is possible to assess and improve the out come of the virtual experience through the PDA/mobile phone, eventually also using information from the bio and activity

sensors.

2. training and homework: Due to the advanced graphic and communication capabilities now available on PDAs/smart phones, they can be used as simulation devices to facilitate the real-world transfer of knowledge acquired in the virtual world.

3. community: The social links created in the virtual world can be continued in the real one even without revealing the real identity of the user; for 
example, I can send an SMS to a virtual friend in my own real context to ask for support.

It is our view that in p-health the creation of a direct link between the real world experience and the virtual one would serve to improve the accessibility of relevant information, the real-time monitoring of relevant health parameters, the motivation for change, the transfer of acquired knowledge in the real world, the social support, and the availability of anonymous expert guidance.

\section{A possible scenario: addiction}

P-health is an approach to health that, in theory, can be used for any kind of health concern. However, to discuss its feasibility, we decided to identify one possible area of intervention: addiction. The term addiction indicates a recurring by an individual to engage in some specific activity despite harmful consequences to the individual's health, mental state, or social life. The term was originally reserved for drug addictions, but it is now also applied to other compulsions such as pathological gambling, compulsive overeating, alcoholism, and so on. Addiction is a disease [54], a state of physiological or psychological dependence on something manifesting as a condition in which medically significant symptoms liable to have a damaging effect are present. Treatment of dependency is usually conducted by a wide range of medical and allied professionals, including addiction medicine specialists, psychiatrists, appropriately trained nurses, social workers, and counselors, and is focused on the individual's ultimate decision to pursue an alternate course of action. Behavioral treatments usually involve the planning of specific ways to avoid the addictive stimulus and therapeutic interventions intended to help a patient learn healthier ways to find satisfaction.

Literature on behavioral analysis and behavioral psychology shows that behavioral therapy, community reinforcement approaches, cue exposure therapy, social skills training, and contingency management strategies are useful approaches for the treatment of addiction [55]. Following these indications, we are developing Eureka [56], a Second Life island for addiction prevention and treatment. Eureka is a virtual immersive environment organized around three different but interconnected areas: the Learning area, the Community area, and the Experience area.

The goal of the Learning area is to use motivation provided by virtual 
worlds to teach users about how to improve their living habits. The Learning area is organized around different learning areas (Figure 1), both without and with teachers (classes). In this area, users learn how to manage daily choices and activities, acquire general and specific information about addiction, and get the information needed to succeed, with daily tips and expert ideas.

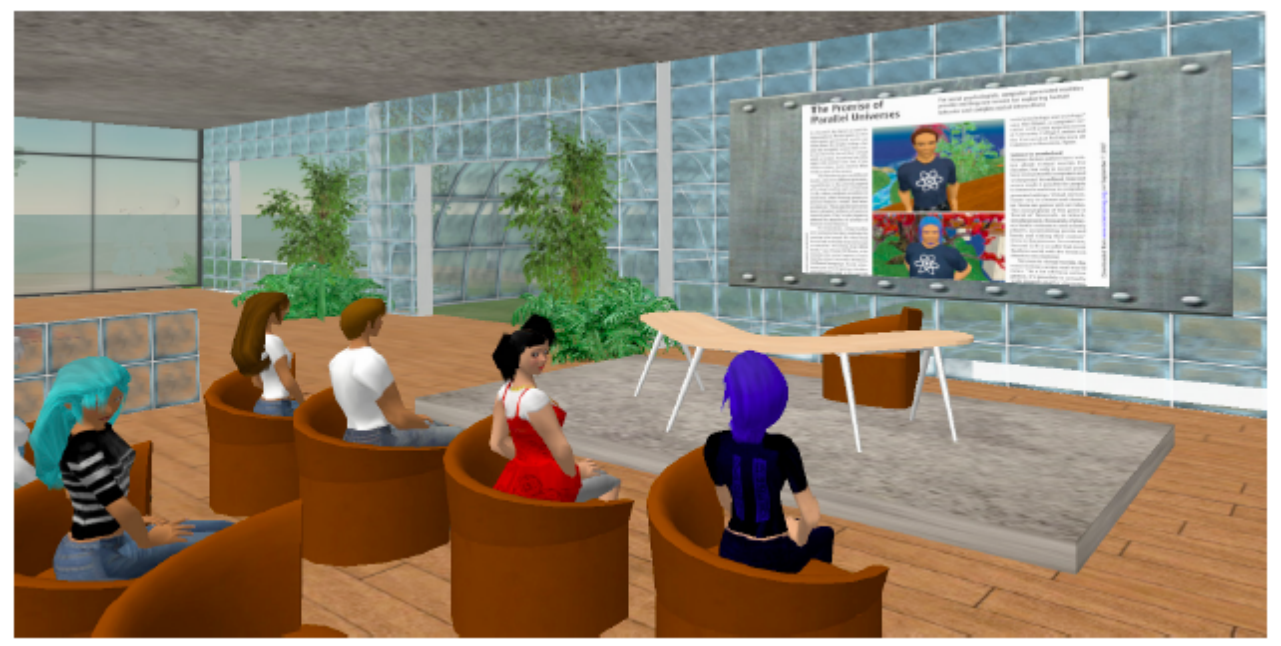

Fig. 1. A screenshot from the Learning area [56].

The goal of the Community area is to use the strength of virtual communities to provide real-life insights aimed at improving living habits. The Community area is organized around different zones (Figure 2) in which users discuss and share experiences among themselves with or without the supervision of an expert (physician, psychologist, nutritionist, etc). 


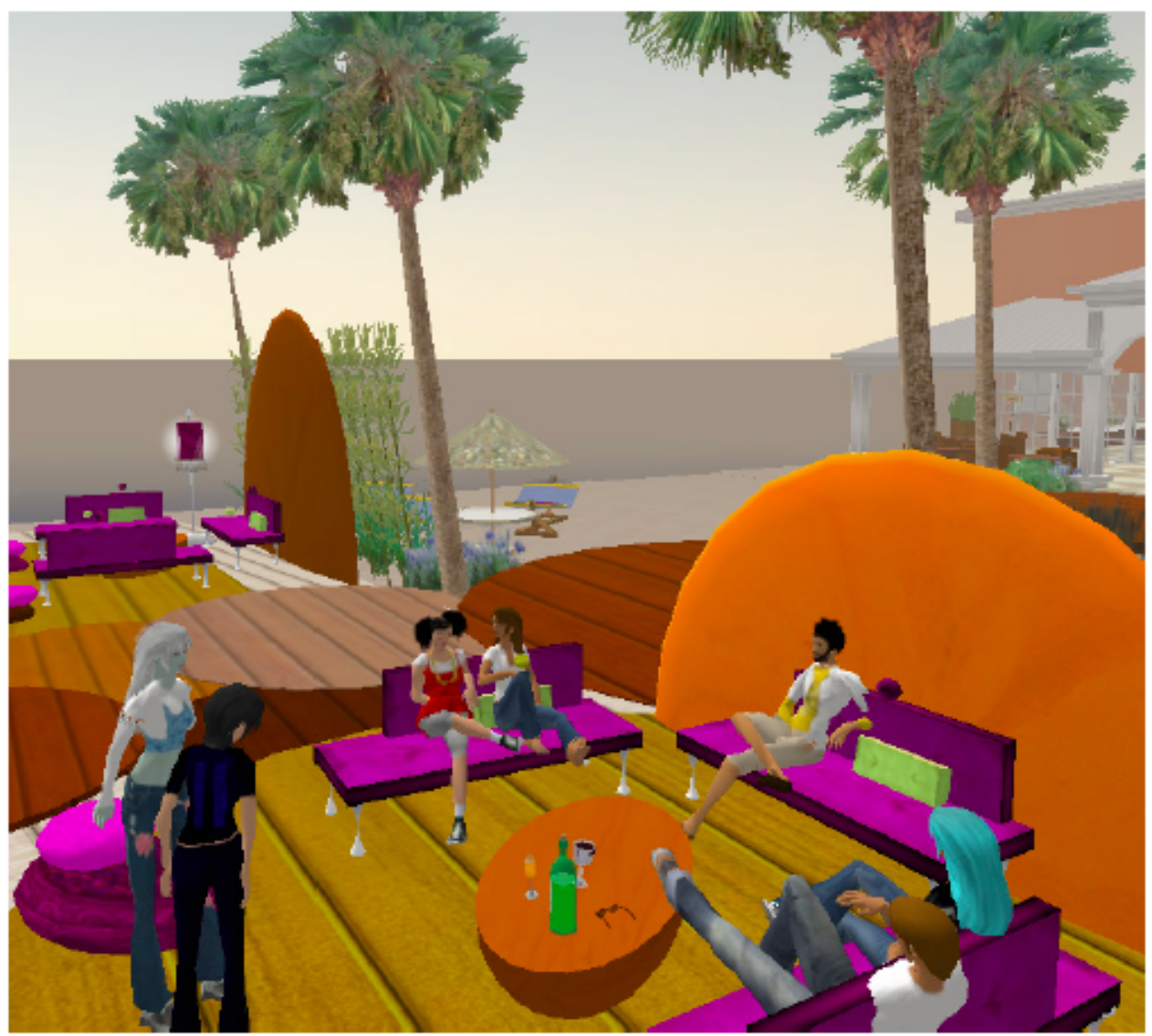

Fig. 2. A screenshot from the Community area.

In the Learning and Community areas, users enjoy support and guidance, learn how to make wise choices and live healthily, and benefit from the exchange of practical experiences and tips from other users.

The goal of the Experience area is to use the feeling of presence provided by the virtual experience to practice both emotional and relational management and general decision-making and problem-solving skills. This area includes different zones (Figure 3) presenting critical situations related to the maintenance and relapse mechanisms (Mall, Supermarket, Pub, Restaurant, Kitchen, etc). Each of these environments is experienced only under supervision. 


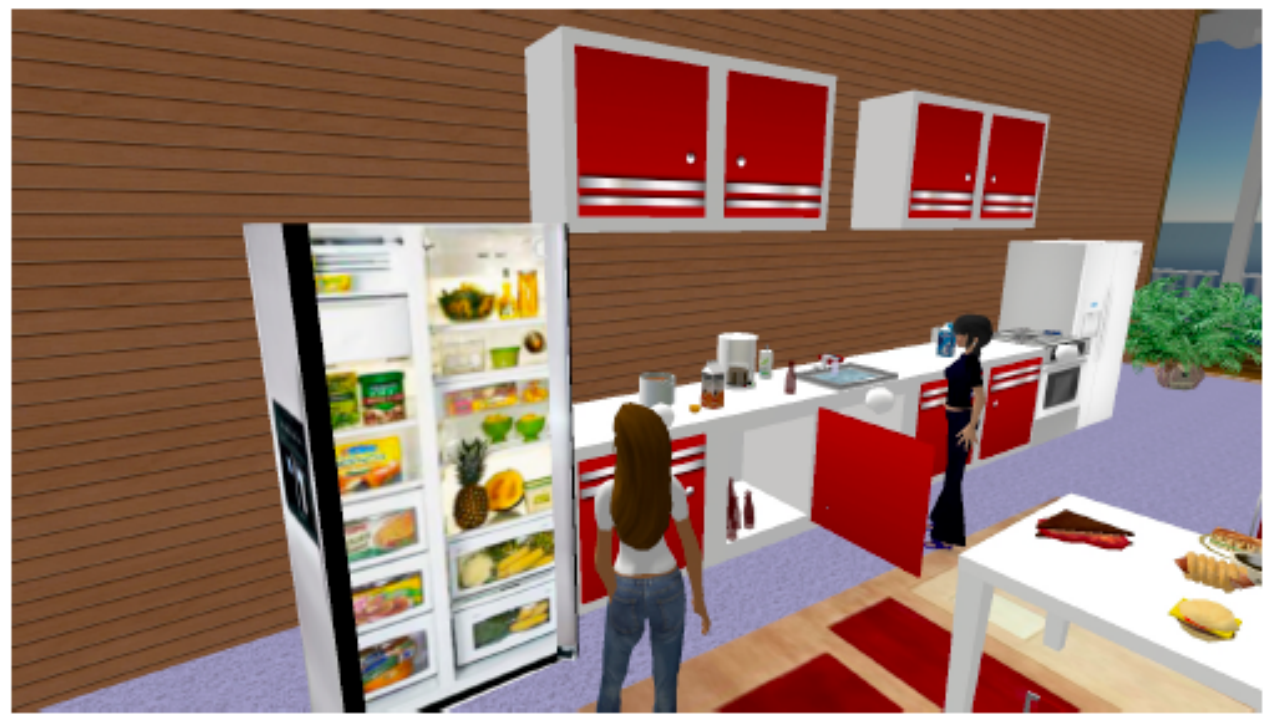

Fig. 3. A screenshot from the Experience area (in particular, the kitchen has been created for people affected by food craving).

In all three of these areas, the user is helped to develop specific strategies for avoiding and/or coping with their problems. After the experience, the coach explores the patient's under standing of what happened in the virtual experience and the specific reactions - emotional and behavioral - to the different situations experienced. If needed, some new strategies for coping with the situations are presented and discussed. In all three areas, type and intensity of care will vary depending on the type of intervention (e.g., prevention vs treatment).

In our vision, Eureka could be an interesting starting point to test the efficacy of online virtual worlds in the prevention and treatment of different psychological disorders.

\section{Conclusions}

This paper addresses a broad and emerging idea in the field of eHealth: the use of 3-D virtual worlds for online mental health applications. As we have recently discussed elsewhere [57], 3-D online worlds have become not only fertile ground for psychologists exploring human behavior [58], but they are also starting to play an emergent role in health services. Why should this be so? Compared with traditional telehealth systems (videoconferencing, email, 
telephone, Web 1.0 applications, etc) and other available technologies (e.g., CD or DVD), 3-D virtual worlds provide users with a more immersive and socially interactive experience, as well as a feeling of embodiment that has the potential to facilitate the clinical communication process and to positively influence group interaction and cohesiveness in group-based therapies. Moreover, unlike the available VR software (see, for example, NeuroVR [59]), 3-D virtual worlds, being Internet-based applications, can be used by different people from different places without physical limitations.

Although this new medium has the potential to improve existing eHealth applications, there are several challenges that need to be addressed. First, more basic psychological research is needed in order to gain a clearer understanding of psychological, communicative, and interpersonal aspects of avatar-based interactions and of the differences between this and other interaction modes. Second, to date, there is scant encouraging data coming from traditional telepsychology applications [60-63] and online communities $[39,44]$ and no experimental or controlled data about the therapeutic effectiveness of online virtual worlds in patients with mental health disorders. Third, 3-D virtual worlds were not created with clinical purposes in mind. This means that clinicians and researchers have to create specific and protected environments to meet their clinical needs as well as the needs of patients. Further, as for any kind of eHealth system, it is important to define international guidelines for the development of 3-D virtual world-based clinical applications in order to reduce the risk of abuse and to guarantee appropriate levels of privacy. Finally, online virtual worlds have open access, meaning that it may be difficult to create safe therapeutic environments in which patients can interact with therapists without external interferences and with privacy protection. Also, cost issues should not be overlooked. The vast majority of virtual worlds have high subscription costs, which may be too expensive for private therapists; in February 2008, the price for an island in Second Life was US \$1675 plus a US \$295 monthly fee. Finally, most online worlds provide users with building tools (editors) that are not easy to use for non-experts as they often require the user to learn script-based programming languages.

In conclusion, despite technical, ethical, and economic issues,

we suggest that 3-D virtual worlds, used as an adjunct to face-to-face settings, may represent a valid opportunity for the future developments in eHealth. Our hope is that the present paper will stimulate a discussion within the research community about the potential, the limitations, and the risks that this emerging medium offers for cybertherapy applications. 


\section{Acknowledgments}

This paper was partially supported by the European Union "Information Society Technologies - IST" Programme through its PASION (Psychologically Augmented Social Interaction Over Networks, IST-27654) and INTREPID (IST-2002-507464) research projects. 


\section{References}

1. Graham P. Web 2.0. 2005. URL:

http://www.paulgraham.com/web20.html [accessed $2008 \mathrm{Jul} 25$ ]

[WebCite Cache ID 5VzpkrigE]

2. Eysenbach G. Medicine 2.0: social networking, collaboration, participation, apomediation, and openness. J Med Internet Res 2008;10(3):e22 (forthcoming). [doi: 10.2196/jmir.1030]

3. Kamel Boulos MN, Wheeler S. The emerging Web 2.0 social software: an enabling suite of sociable technologies in health and health care education. Health Info Libr J 2007 Mar;24(1):2-23. [Medline: 17331140] [doi: 10.1111/j.1471-1842.2007.00701.x]

4. Second Life. URL: http://secondlife.com/ [accessed 2008 Jul 25] [WebCite Cache ID 5VzqNH3yL]

5. There. URL: http://www.there.com/ [accessed 2008 Jul 25] [WebCite Cache ID 5Vzqxg401]

6. Active Worlds. URL: http://www.activeworlds.com/ [accessed 2008 Jul 25] [WebCite Cache ID 5Vzr9TKi3]

7. Second Life - Nutrition Game. URL: http://slurl.com/secondlife/ohio\%25university/161/175/25/ [WebCite Cache]

8. Vital Lab. Second Life Development Service from the VITAL Lab @ Ohio University. Vital Wiki. URL:

http://vital.cs.ohiou.edu/vitalwiki/index.php/Second_Life_\%05\%05

Development [accessed 2008 Jul 25] [WebCite Cache ID 5VzreV6M1]

9. Kemp J. Second Life Heart Murmur Sim video demonstration. 2006. YouTube. URL: http://it.youtube.com/ watch?v=xJY2Iwbzop4 [accessed $2008 \mathrm{Jul}$ 25] [WebCite Cache ID 5Vzs3C2LJ]

10. Second Life - Heart Murmur Sim. URL: http://slurl.com/secondlife/waterhead/124/56/32/ [WebCite Cache]

11. Second Life - Virtual Hallucinations (UC Davis). URL: http://slurl.com/secondlife/sedig/26/45/21/ [WebCite Cache]

12. Yellowlees PM, Cook JN. Education about hallucinations using an internet virtual reality system: a qualitative survey. Acad Psychiatry 2006;30(6):534-539 [FREE Full text] [Medline: 17139026] [doi: 10.1176/appi.ap.30.6.534]

13. Second Life - The Gene Pool in Second Life. URL: http://slurl.com/secondlife/genome/137/87/29 [WebCite Cache] 
14. Second Life - VNEC-Virtual Neurological Education Centre in Second Life. URL: http://slurl.com/secondlife/

Lost $\% 20$ Islands\%20NW/48/83/22 [WebCite Cache]

15. Second Life - Healthinfo Island. URL:

http://slurl.com/secondlife/Healthinfo\%20Island/184/61/22

[WebCite Cache]

16. Boulos MNK, Hetherington L, Wheeler S. Second Life: an overview of the potential of 3-D virtual worlds in medical and health education. Health Info Libr J 2007 Dec;24(4):233-245. [Medline: 18005298] [doi: 10.1111/j.1471-1842.2007.00733.x]

17. van Kokswijk J. Hum@n, Telecoms \& Internet as Interface to Interreality. Hoogwoud, The Netherlands: Bergboek; 2003.

18. Lee KM. Why presence occurs: evolutionary psychology, media equation, and presence. Presence 2004;13(4):494-505. [doi: 10.1162/1054746041944830]

19. Riva G. Virtual reality and telepresence. Science 2007 Nov 23;318(5854):1240-1242. [Medline: 18033867] [doi: $10.1126 /$ science. $318.5854 .1240 \mathrm{~d}]$

20. Riva G, Anguera MT, Wiederhold BK, Mantovani F. From Communication to Presence: Cognition, Emotions and Culture towards the Ultimate Communicative Experience. Amsterdam, The Netherlands: IOS Press; 2006.

21. Zhao S. Toward a taxonomy of copresence. Presence 2003;12(5):445-455. [doi: 10.1162/105474603322761261]

22. Biocca F, Harms C, Burgoon J. Toward a more robust theory and measure of social presence: review and suggested criteria. Presence 2003;12(5):456-480. [doi: 10.1162/105474603322761270]

23. Reeves B. "Being there": Television as Symbolic versus Natural Experience. Stanford, CA: Institute for Communication Research, Stanford University; 1991.

24. Casanueva JS, Blake EH. The Effects of Avatars on Co-presence in a Collaborative Virtual Environment. Technical Report CS01-02-00 TR. Cape Town, South Africa: Department of Computer Science, University of Cape Town; 2001.

25. Schroeder R. The Social Life of Avatars. New York: Springer; 2002.

26. Bente G, Rüggenberg S, Krämer NC. Social presence and interpersonal trust in avatar-based, collaborative netcommunications. In: 7th Annual International Workshop on Presence 2004. Valencia, Spain: Editorial Universidad Politécnica de Valencia; 2004. 
27. Bente G, Rüggenberg S, Krämer NC. Virtual encounters. Creating social presence in net-based collaborations. In: Slater M, editor. 8th Annual International Workshop on Presence. London, UK: University College London; 2005:97-102.

28. Fabri M, Moore DJ, Hobbs DJ. The emotional avatar: nonverbal communication between inhabitants of collaborative virtual environments. In: Braffort A, editor. Gesture-Based Communication in Human-Computer Interaction: Springer Lecture Notes in Artificial Intelligence 1739. New York: Springer; 1999:245-248.

29. Yee N, Bailenson JN, Urbanek M, Chang F, Merget D. The unbearable likeness of being digital: the persistence of nonverbal social norms in online virtual environments. Cyberpsychol Behav 2007 Feb;10(1):115-121. [Medline: 17305457] [doi:

10.1089/cpb.2006.9984]

30. Gorini A, Riva G. Virtual reality in anxiety disorders: the past and the future. Expert Rev Neurother 2008 Feb;8(2):215-233. [Medline: 18271709] [doi: 10.1586/14737175.8.2.215]

31. Riva G, Gaggioli A, Villani D, Preziosa A, Morganti F, Corsi R, et al. NeuroVR: an open source virtual reality platform for clinical psychology and behavioral neurosciences. Stud Health Technol Inform 2007;125:394-399. [Medline: 17377310]

32. Pull CB. Current status of virtual reality exposure therapy in anxiety disorders: editorial review. Curr Opin Psychiatry 2005 Jan;18(1):714. [Medline: 16639177]

33. Allison SE, von Wahlde L, Shockley T, Gabbard GO. The development of the self in the era of the internet and role-playing fantasy games. Am J Psychiatry 2006 Mar;163(3):381-385 [FREE Full text] [Medline: 16513856] [doi: 10.1176/appi.ajp.163.3.381]

34. Rizzo A, Pair J, McNerney PJ, Eastlund E, Manson B, Gratch J, et al. Development of a VR therapy application for Iraq war military personnel with PTSD. Stud Health Technol Inform 2005;111:407413. [Medline: 15718769]

35. Wiederhold BK, Wiederhold MD. Virtual Reality Therapy for Anxiety Disorders. Washington, DC: American Psychological Association Press; 2004.

36. Winkelman WJ, Choo CW. Provider-sponsored virtual communities for chronic patients: improving health outcomes through organizational patient-centred knowledge management. Health Expect 2003 Dec;6(4):352-358. [Medline: 15040797] [doi: 10.1046/j.1369-7625.2003.00237.x] 
37. Wenger E. Communities of Practice: Learning, Meaning, and Identity. Cambridge, UK: Cambridge University Press; 1998:318.

38. Fildes J. Virtual world 'tackles paranoia'. BBC. 2003. URL: http://news.bbc.co.uk/2/hi/health/3251365.stm [accessed $2008 \mathrm{Jul}$ 25] [WebCite Cache ID 5VztrlCVU]

39. Fildes J. Virtual treatment for US troops. BBC. 2007. URL: http://news.bbc.co.uk/2/hi/science/nature/6375097.stm [accessed 2008 Jul 25] [WebCite Cache ID 5VztcArDh]

40. Lester J. About Brigadoon. Brigadoon: An Innovative Online Community for People Dealing with Asperger's Syndrome and Autism. Braintalk Blog. URL:

http://braintalk.blogs.com/brigadoon/2005/01/about_brigadoon.html [accessed 2008 Jul 25] [WebCite Cache ID 5ZdXqHvIx]

41. Biever C. Web removes social barriers for those with autism. 2007. New Scientist. URL: http://technology.newscientist.com/ channel/tech/mg19426106.100-web-removes-social-barriers-forthose-with-autism.html [accessed 2008 Jul 25] [WebCite Cache ID 5VzuV4H7B]

42. Brady J. How 'Second Life' therapy helps Asperger's patients. 2008. WFAA TV News. URL:

http://www.wfaa.com/sharedcontent/dws/wfaa/localnews/news8/stor ies/wfaa080111_lj_brady.11fb5bac.html [accessed 2008 Jul 25] [WebCite Cache ID 5Vzu9DCSQ]

43. Second Life - Autistic Liberation Front in Second Life. URL: http://slurl.com/secondlife/Porcupine/29/177/113 [WebCite Cache]

44. Second Life - Live2give. URL:

http://slurl.com/secondlife/144/210/28 [WebCite Cache]

45. Lester J. All about Live2Give. Live2Give: An innovative Online Community for people dealing with Cerebral Palsy and similar conditions. Braintalk Blog. URL:

http://braintalk.blogs.com/live2give/2005/01/all_about_live2.html [accessed 2008 Jul 25] [WebCite Cache ID 5ZdXuH9Wf]

46. Terdiman D. Second Life Teaches Life Lessons. Second Life Herald. 2005. URL: http://www.secondlifeherald.com/slh/2007/02/interview [accessed 2008 Apr 10] [WebCite Cache ID 5VzvNQHyR]

47. Aquacade A. Interview With the Agoraphobic: Fighting the Fear in Second Life. Second Life Herald. 2007. URL: http:/ /www.secondlifeherald.com/slh/2007/02/interview_with_1.html [accessed 2008 Aug 2] [WebCite Cache ID 5ZnYhV7 $7 \mathrm{w} \overline{\mathrm{V}}$ ]

48. Second Life - Agoraphobia Support Headquarters. URL: 
http://slurl.com/secondlife/Neptune/128/110/30 [WebCite Cache]

49. Luo JS. Virtual reality therapy: on your desktop today. Primary Psychiatry 2007;14:20-22.

50. Glueckauf RL, Pickett TC, Ketterson TU, Loomis JS, Rozensky RH. Preparation for the delivery of telehealth services: a self-study framework for expansion of practice. Prof Psychol: Res Pract 2003;34(2):159-163. [doi: 10.1037/0735-7028.34.2.159]

51. Koocher GP, Morray E. Regulation of telepsychology: a survey of state attorneys general. Prof Psychol: Res Pract 2000;31(5):503-508. [doi: 10.1037/0735-7028.31.5.503]

52. Jerome LW, DeLeon PH, James LC, Folen R, Earles J, Gedney JJ. The coming of age of telecommunications in psychological research and practice. Am Psychol 2000 Apr;55(4):407-421. [Medline: 10812693] [doi: 10.1037/0003-066X.55.4.407]

53. American PAEC. Services by Telephone, Teleconferencing, and Internet. Washington, DC: APA; 1997.

54. American Psychiatric Association. Diagnostic and Statistical Manual of Mental Disorders. Fourth Edition (Text Revision). Washington, DC: APA; 2000.

55. O'Donohue W, Ferguson KE. Evidence-based practice in psychology and behavior analysis. Behav Analyst Today 2006;7:335-350.

56. Second Life - Eureka. URL: http://slurl.com/secondlife/eureka/203/113/29 [WebCite Cache]

57. Gorini A, Gaggioli A, Riva G. Virtual worlds, real healing. Science 2007 Dec 7;318(5856):1549. [Medline: 18063769] [doi:

$10.1126 /$ science. $318.5856 .1549 \mathrm{~b}]$

58. Miller G. Living in societies. The promise of parallel universes. Science 2007 Sep 7;317(5843):1341-1343. [Medline: 17823341] [doi: 10.1126/science.317.5843.1341]

59. NeuroVR. URL: http://www.neurovr.org/ [accessed 2008 Jul 25] [WebCite Cache ID 5VzvaCHeA]

60. Alcañiz M, Botella C, Baños R, Perpiñá C, Rey B, Lozano JA, et al. Internet-based telehealth system for the treatment of agoraphobia. Cyberpsychol Behav 2003 Aug;6(4):355-358. [Medline: 14511446] [doi: 10.1089/109493103322278727]

61. Goldfield GS, Boachie A. Delivery of family therapy in the treatment of anorexia nervosa using telehealth. Telemed J E Health 2003;9(1):111-114. [Medline: 12699614] [doi:

10.1089/153056203763317729]

62. Miller TW, Kraus RF, Kaak O, Sprang R, Burton D. Telemedicine: a 
child psychiatry case report. Telemed J E Health 2002;8(1):139-141. [Medline: 12020414] [doi: 10.1089/15305620252933482]

63. Nelson E, Barnard M, Cain S. Treating childhood depression over videoconferencing. Telemed J E Health 2003;9(1):49-55. [Medline: 12699607] [doi: 10.1089/153056203763317648]. 



\section{CHAPTER IX}

Summary and concluding remarks 

The aim of the present thesis was to investigate the effectiveness of VR as an innovative tool to assess and treat anxiety (Tab. 1 summarizes the key issues discussed in the different chapters).

\section{KEY ISSUES}

- VR based treatments represent a new emerging treatment for anxiety that integrates different virtual reality experiences with traditional cognitivebehavioral techniques.

- VR based treatments consist on the use of computer-generated environments to recreate the feared and stressful stimuli and provide the opportunity for habituation in a controlled and safe environment.

- The feeling of actual presence offered by VR allows patients to experience the exposure in a more vivid and realistic manner than they could do through their own imagination.

- The therapist sees what the patient sees. In this way it is easy to identify and monitor the patient's reactions. To the different stimuli.

- The use of VR seems to increase the patients' adherence to treatment

- VR based treatments provide graded exposure so that patients can start at the easiest level and progress to the most difficult.

- VR is an efficient distraction tool for patients exposed to stressful situations.

- Different controlled studies have verified the efficacy of VR based treatments in the treatment of anxiety disorders.

- The diffusion of Web 2.0 has facilitated the creation of customized, online virtual environments incredibly less expensive than off-line VR software.

Tab. 1. A brief summary of the key issues discussed in the different chapters. 
After a general introduction on virtual reality (Chapter one), we discussed the role of immersion and narrative in eliciting the sense of presence observing that both these components are important to create an effective virtual reality experience (Chapter two).

The third chapter was then mainly devoted to experimentally verify the hypothesis that virtual stimuli can really replace the real ones in exposurebased approaches, and that they are more effective than static pictures in eliciting emotional responses in users. Even if never directly experimentally demonstrated, this hypothesis represents the premises of any clinical protocol that uses virtual exposure instead of in-vivo exposure to treat different kinds of anxiety disorders. As expected, we found that VR is effective as in-vivo exposure, and more effective than exposure to static pictures in eliciting a psychological and physiological response of anxiety in patients (i.e. patients affected by eating disorders) when exposed to the stimuli that are critical for their disease (i.e. high-calorie food).

Even if obtained from a very specific category of patients, these findings support the use of VR as an efficient and ecological clinical instrument that induces emotional responses very similar to those induced by real life contexts. Using virtual environments we can perform sophisticated neuropsychological tests, such as the C-G Arena (see Chapter four) in a very controlled and replicable situation.

Chapter five, six and seven are mainly devoted to demonstrate the efficacy of VR as a more efficacious instrument than pure imagination and traditional relaxing and distraction techniques (such as music or meditation) to help patients to reduce their pathological or situational anxiety. In particular, when used in patients who suffer from excessive anxiety caused by the fear of undergoing a surgical operation under local anesthesia, VR provides a particularly intense form of immersive cognitive and emotional distraction that taxes the patient's limited attention capacity, resulting in the withdrawal of attention from the real, noxious, external stimuli with a subsequent reduction in perceived pain and stress ${ }^{1-3}$.

Our data, together with the rest of the literature in the field, strongly supports the use of VR as empowering tool for personal change. The concept of empowerment includes the expansion of freedom of subject's choice and action, that is the increasing of the subject's authority and control over the resources and decisions that affect his/her life. When we make real choice, we gain increased control over our lives and are able to change ourselves. However, many subjects have great difficulties in exercising effective choices: they often do not have knowledge, skills, assertiveness, or self esteem needed to make the correct choice. In these 
cases an artificial reality that projects the user into a 3D space generated by the computer, may offer him/her an enriched experience under the full control of the therapist that transforms VR in an "empowering environment", a special, sheltered setting where patients can start to act, experiment and think without feeling actually threatened.

Thus, although it is indisputable that VR has come of age for research and clinical applications, there are still some important limitations that should be overcome for its use in the daily clinical practice ${ }^{4}$ :

- the lack of standardization in VR hardware and software, and the limited possibility of tailoring the virtual environments to the specific requirements of the clinical or experimental setting ${ }^{5}$;

- the lack of accepted standards for the ergonomic/usability evaluation of virtual environments given the clinical nature of the applications and users ${ }^{6}$; - the low availability of standardized protocols that can be shared by the community of researchers;

- the high costs required for designing and testing a new clinical VR application;

- the fact that most of the existing VR applications for mental health run on a single PCs located in the office of the therapists or in the research labs.

However, as we have discussed in Chapter eight, the enormous diffusion of the World Wide Web together with the introduction of the Web 2.0 have enormously facilitated the inexpensive creation of customized virtual worlds accessible from any computer connected to Internet, as well as the development of new forms of collaborative interactions between multiple users, even if physically distant. Compared with conventional VR systems, 3D shared virtual worlds like Second Life (http://www.secondlife.com) may convey greater feelings of presence, facilitate the clinical communication process, positively influence group cohesiveness in group-based therapies, and foster higher levels of interpersonal trust between therapists and patients $^{7,8}$.

\section{Possible limitations in the use of VR}

Even if short, it could be useful to make a list of the possible contraindications that can emerge from the use of VR.

Some medical conditions represent a risk for the use of VR: the presence of migraine, headache, seizure disorder and vestibular abnormalities must be investigated before VR exposure. There is also some evidence that the use 
of 3D environments provokes changes in heart rate, and increases systolic and diastolic blood pressure and oxygen consumption, suggesting caution when these tools are used with patients with hypertension, cardiovascular and circulatory diseases. In addition, since VR might interfere with normal psychological processes, a careful observation is necessary when using it with patients with schizophrenia, or with serious personality disorders who are pathologically predisposed to become confused by real versus virtual worlds ${ }^{9}$.

\section{Contraindications}

\section{Cybersickness}

There is a tendency for some VR users to exhibit symptoms that parallel those of classical motion sickness both during and after the virtual experience. Cybersickness is distinct from motion sickness in that the user is often stationary but has a compelling sense of self-motion through moving visual imagery. The symptoms related to cybersickness regard different target areas: visual (visual blurring, double vision, tearing, irritation redness), auditory (tinnitus and decreased hearing), vestibular (dizziness, nausea, vomiting and sweating), CNS (headache, seizures, flashbacks, disorientation and instability), musculoskeletal (neck strain, wrist strain and back pain).

Unfortunately, there is currently no foolproof method for eliminating the problem, but a gradual introduction to virtual environments and shorter exposure time can help to prevent symptoms. Several existing questionnaires can be administered to assess for the risk of cybersickness before the beginning of the treatment. The most commonly used, the Simulator Sickness Questionnaire, has been shown to be a reliable indicator for predicting cybersickness in military pilots and in undergraduate populations ${ }^{10}$. This questionnaire is a reasonable starting point for the assessment of discomfort in patients.

\section{Long-term effects}

Very little is known about the long-term effects of VR exposure. Ungs reported that a very small number of subjects ( $4.6 \%$ of the sample) experienced VR side effects, including visual flashbacks, balance disorder and lack of hand-eye coordination or longer than $24 \mathrm{~h}$ after the conclusion of the session ${ }^{11}$. In some cases, VR induces subjects to confuse the virtual experience with the real one, resembling the effects of drugs such as hallucinogens ${ }^{12}$. When this happens, the main risk is that people may prefer 
the virtual world to the real one and completely withdraw from society or, if the sense of reality becomes blurred, patients may become unable to distinguish safe from dangerous behaviors.

Addiction and social isolation are other risks that are connected to the use of VR, but they are more frequent in children and adolescents ${ }^{13}$.

\section{Looking forward to the future}

Looking at the future we can think to two different possible critical developments of VR. One is related to the continue and fast technological advances that will allow the creation of complex VR systems that, projecting artificial stimuli upon the five senses, will incrementally reshape our way of life, our social interactions, and possibly also the therapeutic approaches to mental disorders. These systems will be also integrated one with the others in a way in which their content can be moved across platforms and where separate worlds can be linked together, progressively increasing our daily use of technology. Moreover, the integration of these "parallel" worlds with new input/output technologies, such as braincomputer interfaces systems, will dramatically change the way we interact with the virtual environments, in particular facilitating the use of technology by those persons with important physical disabilities.

In a more clinical perspective, the second important possible development of the virtual reality systems regards the creation of personalized virtual trainings that combine the traditional therapeutic approaches with a hybrid, closed-loop empowering experience bridging real and virtual worlds. Using portable devices, such as mobile phones and PDAs (Personal Digital Assistant) the patient's psychopysiological reactions occurring in the reallife will be recorded by a fusion module that analyzes and sends them to the therapist's computer. The collected information will be then used to modify the virtual environments according to the needs of the single patient in order to give them the possibility to exercise his/her coping abilities during the following virtual sessions (this kind of personalized virtual experience is the core objective of the "Interstress Project", recently funded by the Seventh EU Framework Programme and coordinated by Istituto Auxologico Italiano).

In conclusion, despite any possible issue related to the use of a new technological tool, we suggest that VR, used in respect of the basic psychological principles and in adjunct to face-to-face settings, may 
represent a valid and very promising opportunity to complement and improve the efficacy of existing clinical approaches.

Our hope is that the papers collected in the present thesis will contribute to the ongoing discussion within the clinical community about the potential applications, and also the eventual risks that VR can offer to clinicians and therapists. 


\section{References}

1. Gold JI, Belmont KA, Thomas DA. The neurobiology of virtual reality pain attenuation. Cyberpsychology \& Behavior 2007; 10(4):536-44.

2. Riva G. (2006) Being-in-the-world-with: presence meets social and cognitive neuroscience. In: Riva G, Anguera MT, Wiederhold BK, Mantovani F (eds). From communication to presence: cognition, emotions and culture towards the ultimate communicative experience Festschrift in honor of Luigi Anolli. Amsterdam: IOS Press, 47-80.

3. Wismeijer AA, Vingerhoets AJ. The use of virtual reality and audiovisual eyeglass systems as adjunct analgesic techniques: a review of the literature. Ann Behav Med 2005; Dec; 30(3):268-78.

4. Riva G, Botella C, Légeron P, Optale G (eds). Cybertherapy: Internet and Virtual Reality as Assessment and Rehabilitation Tools for Clinical Psychology and Neuroscience. Amsterdam: IOS Press; 2004.

5. Botella C, Baños R, Guerrero B. Using a flexible virtual environment for treating a storm phobia. Psychnology Journal 2006; 4(2):129-44.

6. Galimberti C, Belloni G, Cantamesse M. The development of an integrated psychosocial approach to effective usability of 3D Virtual Environments for Cybertherapy. Psychnology Journal 2006; 4(2):161-80.

7. Gorini A, Gaggioli A, Riva G. Virtual worlds, real healing. Science 2007; Dec 7; 318(5856):1549.

8. Gorini A, Gaggioli A, Vigna C, Riva G. A Second Life for eHealth: Prospects for the Use of 3-D Virtual Worlds in Clinical Psychology. Journal of Medical Internet Research 2008; 10(3):e:21.

9. Cartwright GF. Virtual or real? The mind in cyberspace. The Futurist 1994; 28(2):22-6.

10. Stanney KM, Kennedy RS. The psychometrics of cybersickness. Commun ACM 1997; 40(8):67-8.

11. Ungs TJ. Simulator induced syndrome: evidence for long-term aftereffects. Aviat Space Environ Med 1989; 60(3):252-5.

12. Mantovani G. Virtual reality as a communication environment: consensual hallucination, fiction, and possible selves. Hum Relat 1995; 48:669-83. 
Chapter IX

13. Plusquellec M. Are virtual worlds a threat to the mental health of children and adolescents? Arch Pediatr 2000; 7(2):209-10. 


\section{APPENDIX A}

\section{Virtual Reality for the treatment of anxiety disorders}

From: A. Gorini, G. Riva. Virtual Reality in anxiety disorders: the past and the future.

Expert Review of Neurotherapeutics. 2008, 8(2): 215-233. 



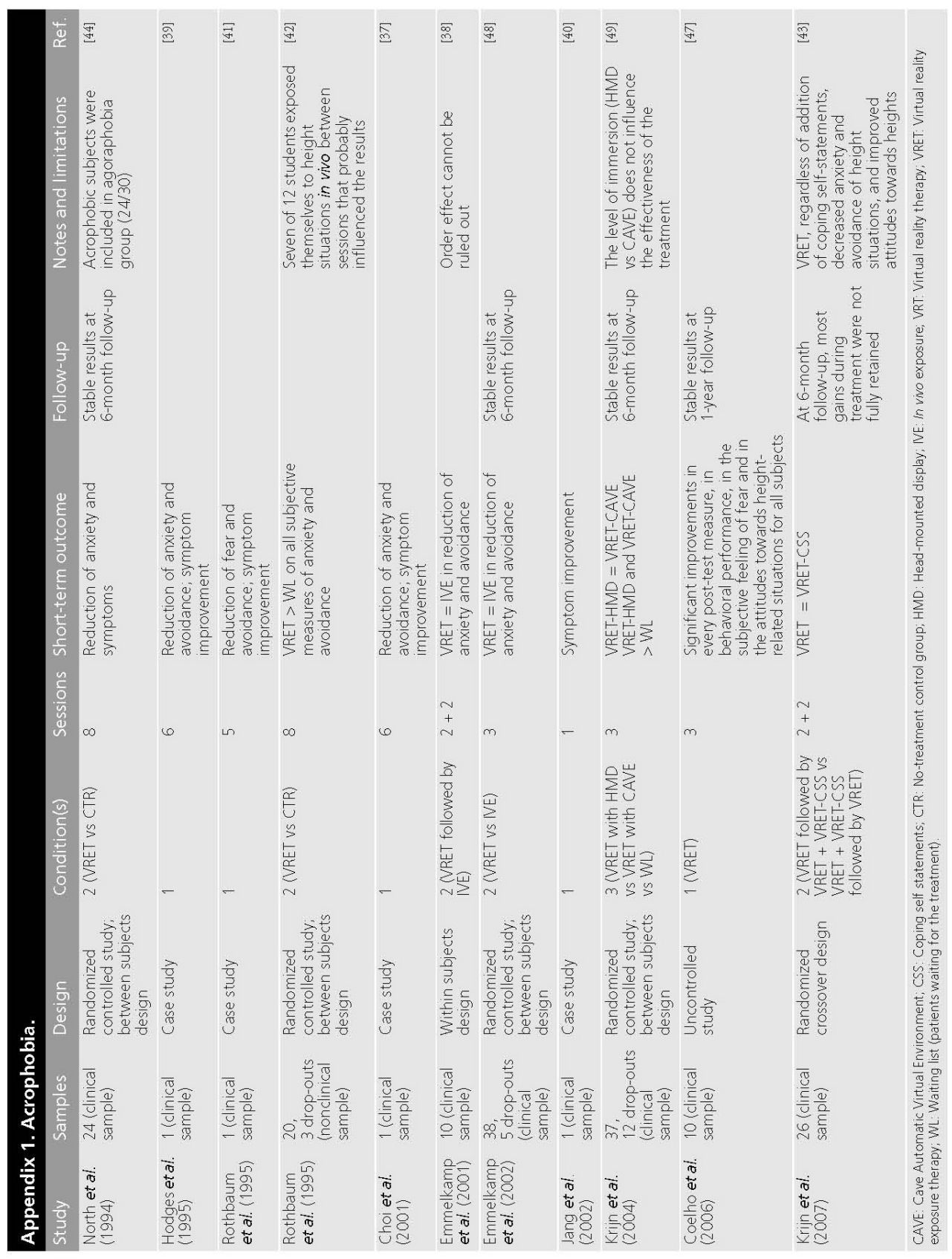




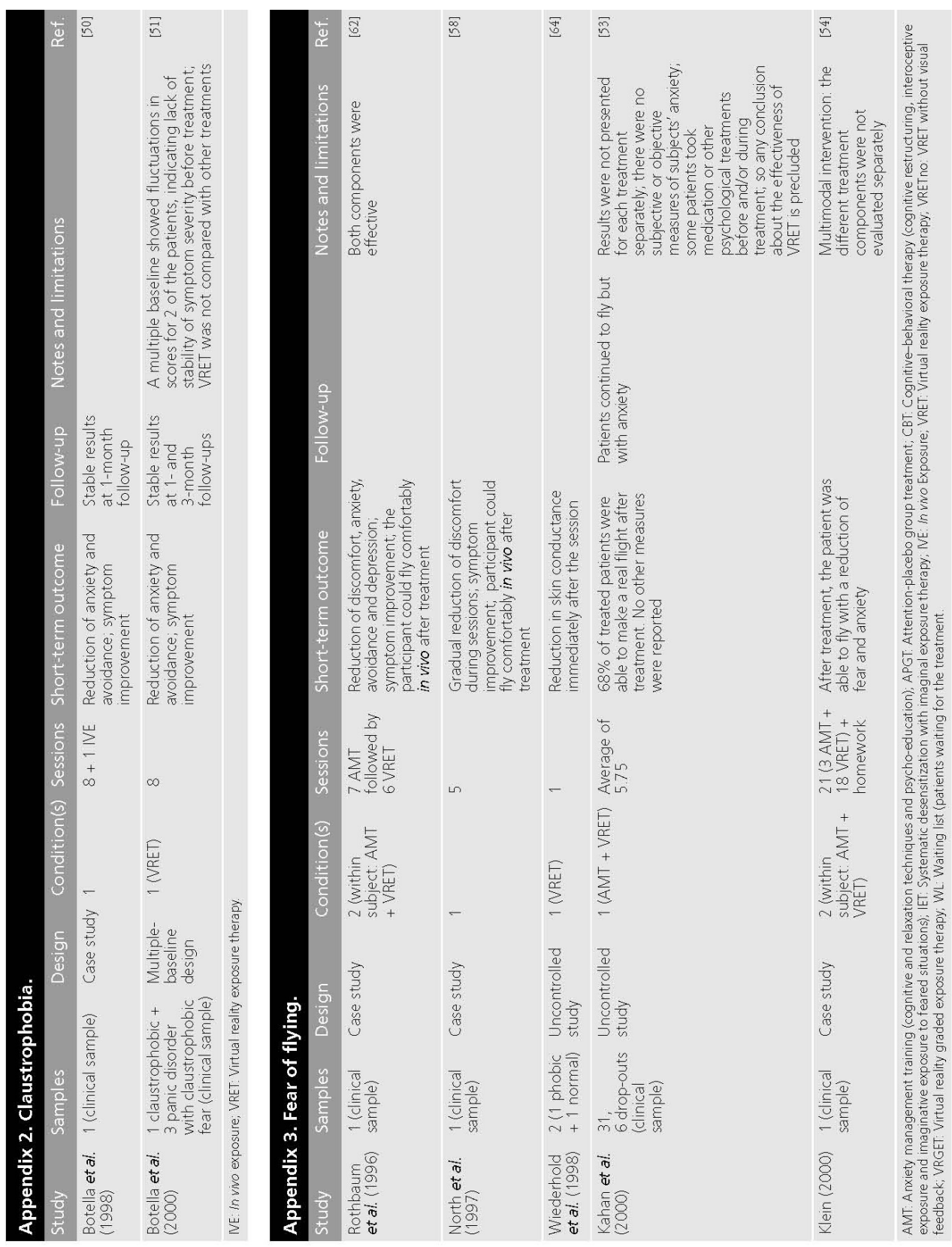




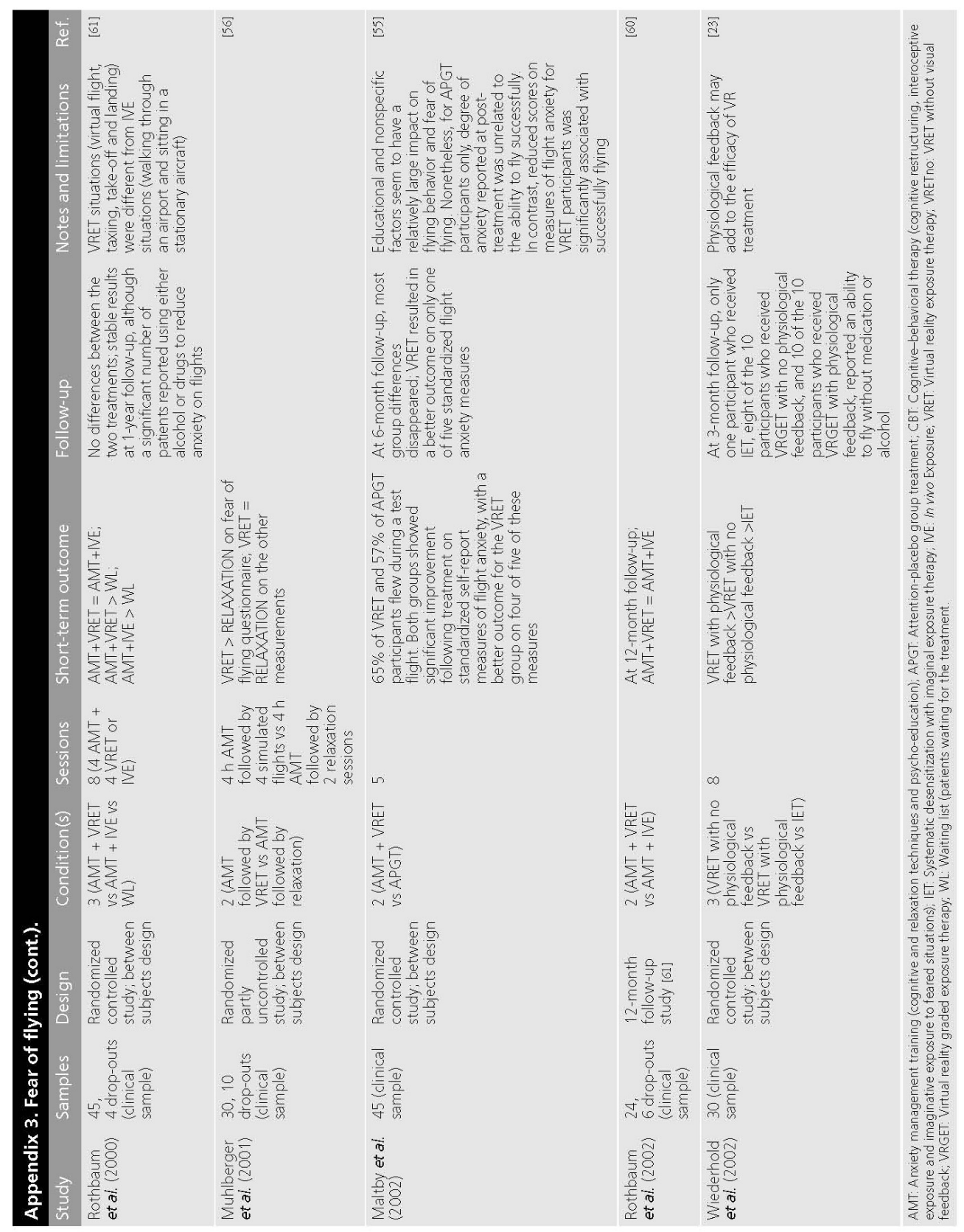




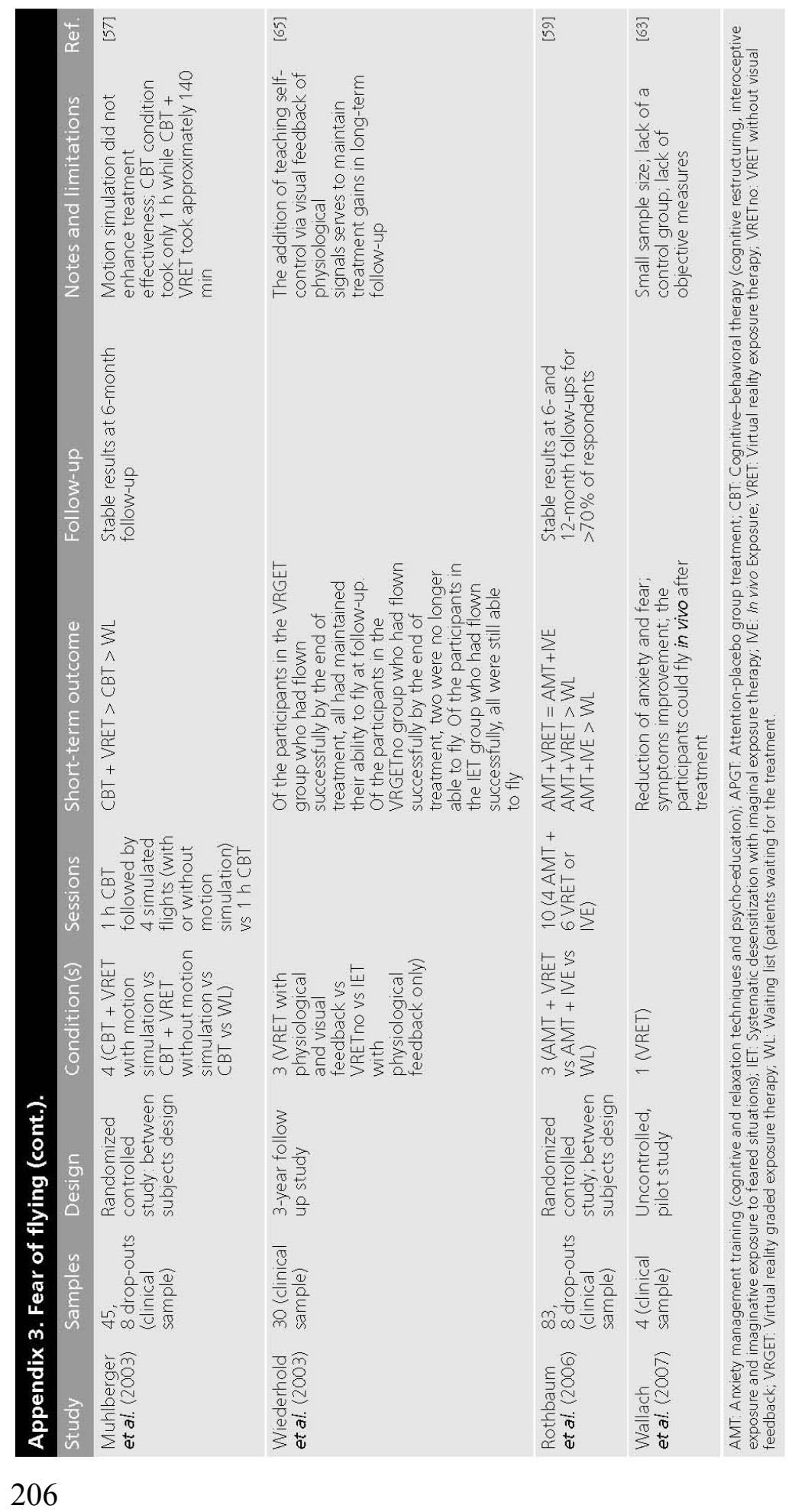



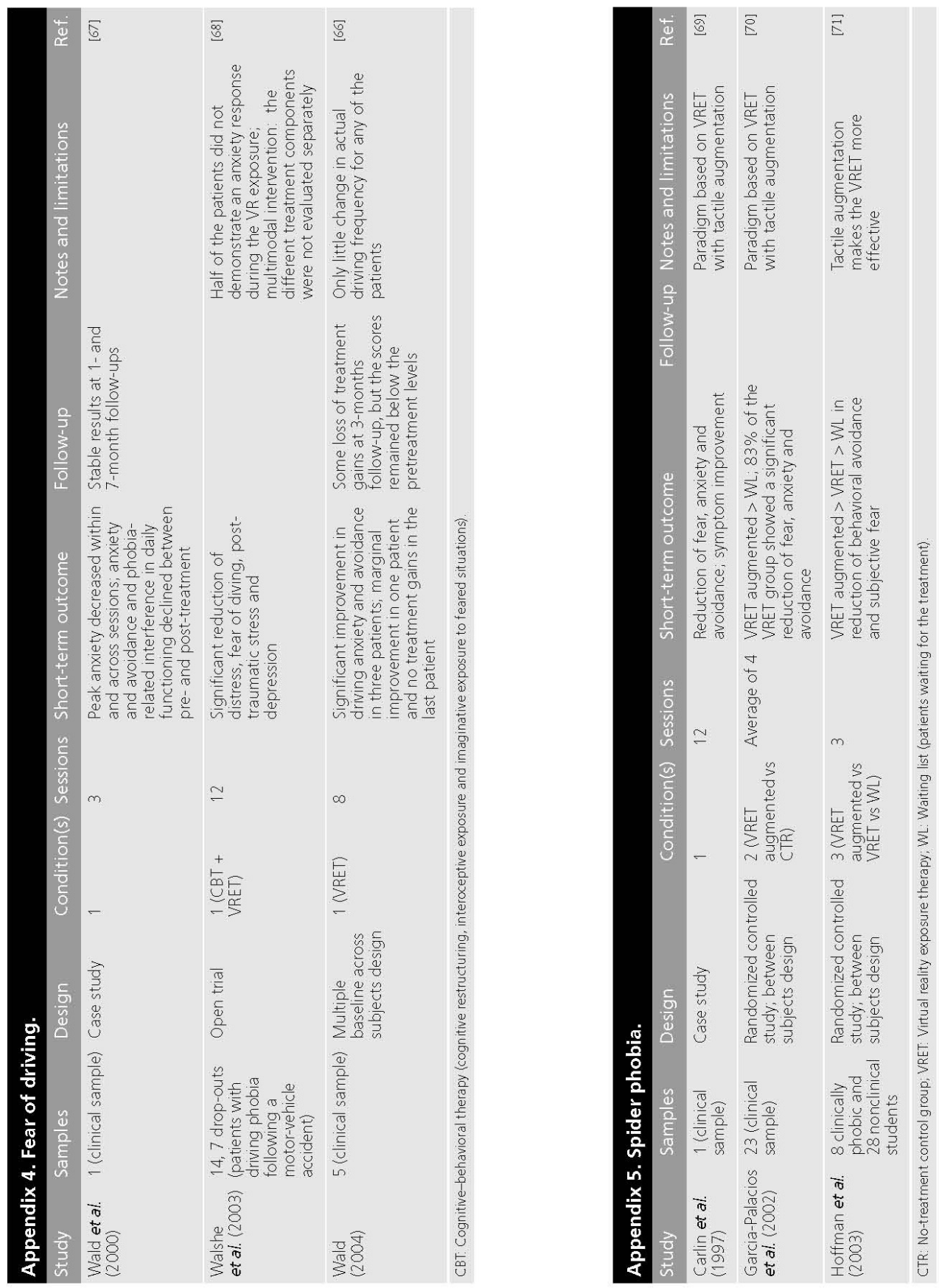


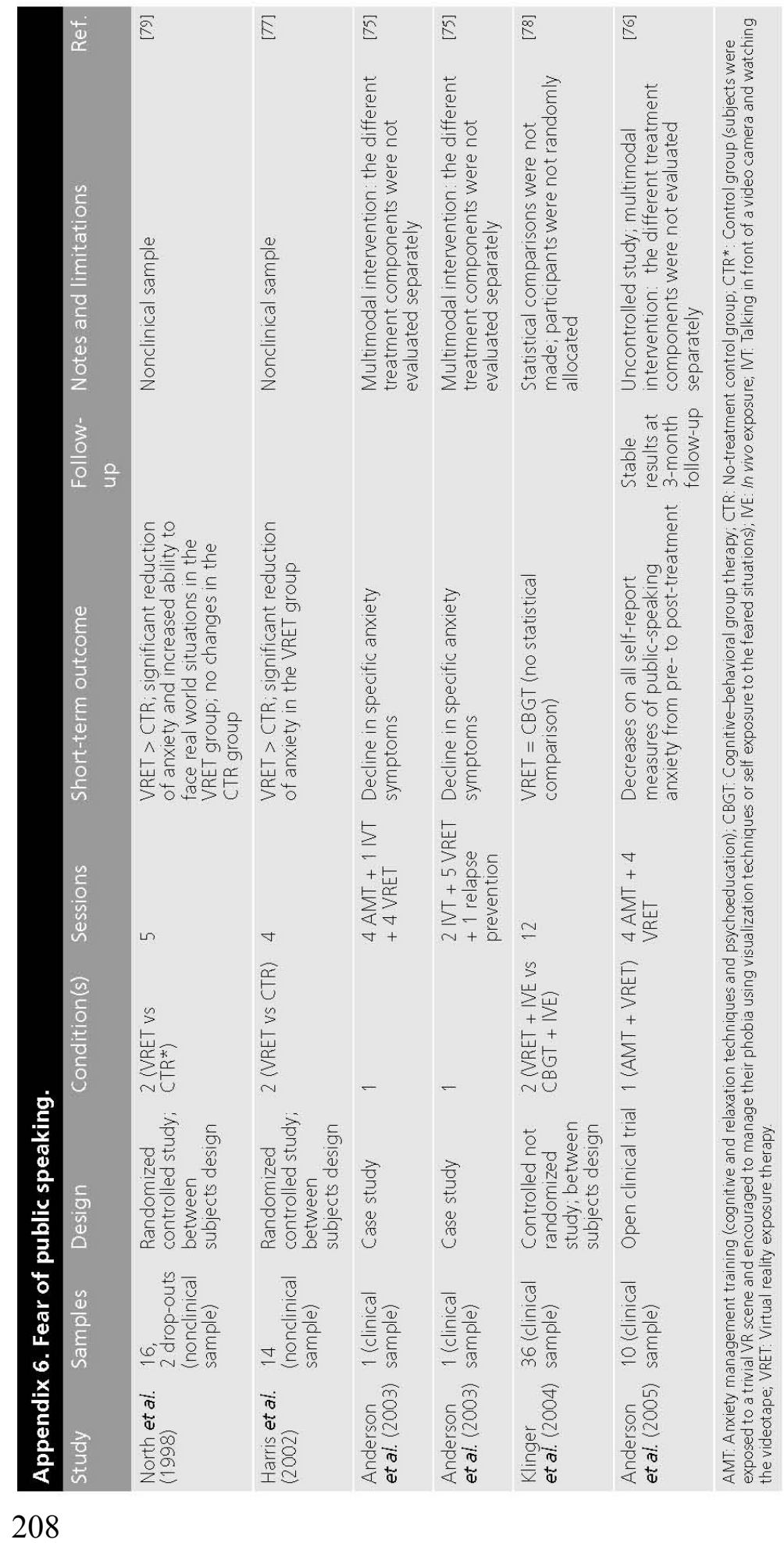




\section{Appendix A}

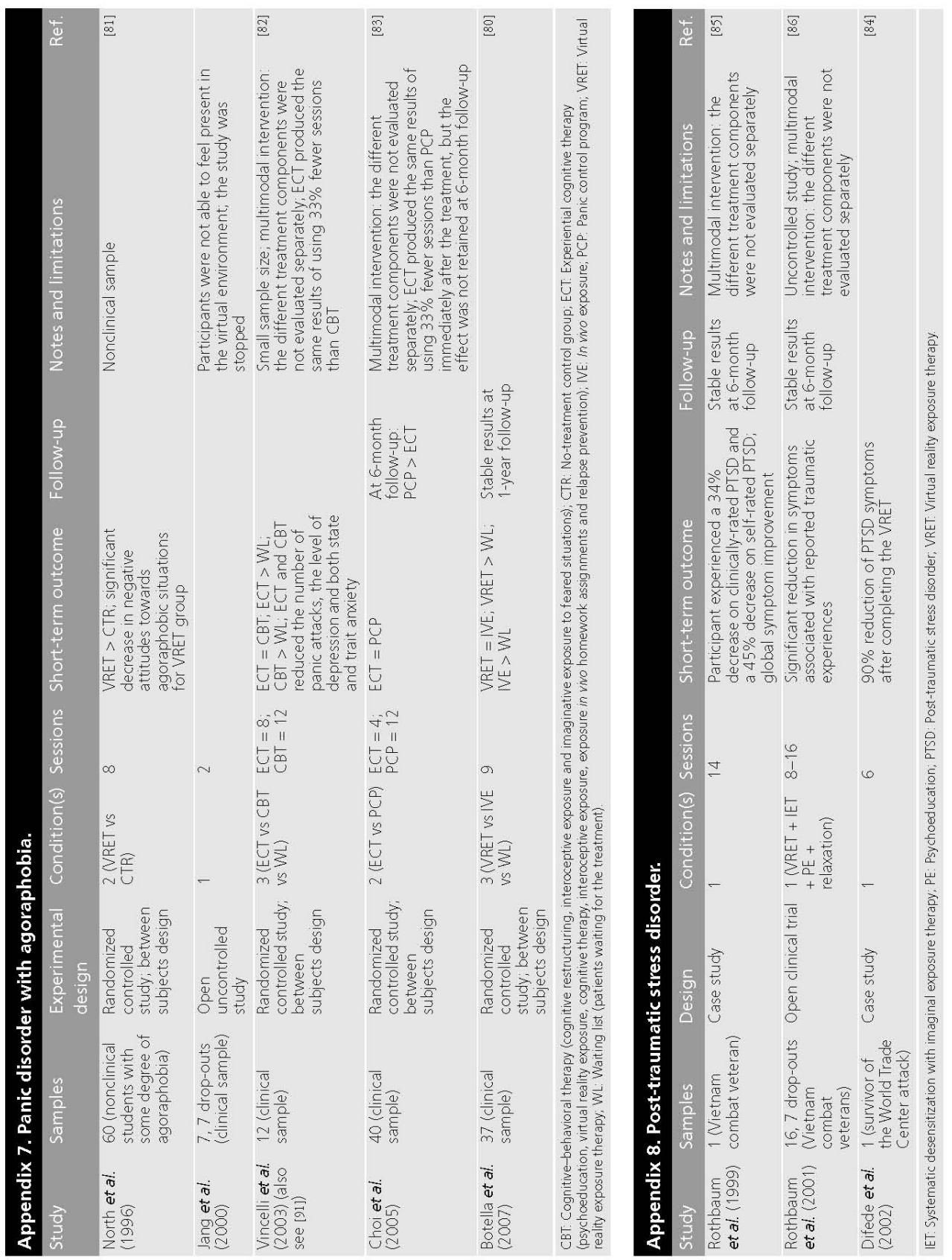



Summary 

Virtual Reality (VR) is an advanced form of human-computer interface that allows users to interact with and become immersed in a computer generated environment in a naturalistic way. Starting from about twenty years ago, clinical psychologists have used VR for the treatment of different psychological disorders taking advantage of the possibility offered by it to create fully controlled environments in which patients can be exposed to anxious stimuli under the direct supervision of their therapists.

The present thesis is focused on some very recent experimental studies aimed to investigate the characteristics of VR as a clinical tool for the treatment of stress and anxiety.

Chapter one provides a general introduction about VR: its technological components are described in details together with a brief history of its applications in clinical practice. The rationale about the use of VR in healthcare is explained, and the advantages of using it for the treatment of anxiety are described.

The following two chapters discuss the psychological aspects involved in a virtual experience. In particular, Chapter two introduces the concept of presence, a psychological construct that refers to the illusion of being present in one (simulated) place when one is actually present in another (physical) place. Presence is a key feature of any virtual experience and is generated by different technological, cognitive and emotional factors. In this first study we tested how to optimize the virtual experience and to increase the sense of presence manipulating some of these factors. Specifically, we tested if an immersive technology and/or a meaningful narrative context influence the users' sense of presence providing a more compelling experience than a non-immersive and non-contextualized virtual space. As expected, we found that both immersion and narrative are important to create an effective virtual reality experience, differently contributing to increase the sense of presence. Immersion increases the place illusion, while the narrative contributes to generate an emotional response and to strengthen the subjects' sense of inner presence.

Chapter three is aimed to experimentally verify the theoretical rationale behind the 'virtual approach', that is that real and virtual exposures elicit a comparable emotional reaction in users. Exposing two samples of patients affected by eating disorders to real food, virtual food and photographs of food, we found that the virtual exposure elicits emotional responses comparable to those produced by the real exposure, and that the sense of presence induced by VR immersion makes the virtual experience more 
ecological, and consequently more effective than static pictures in producing emotional responses in humans. The importance of this study derives from the fact that it is the first attempt, in the entire VR literature, to experimentally compare the effects produced by real and virtual exposure.

From Chapter four to Chapter seven we described four studies in which VR has been used to evaluate and treat different psychological disorders related to stress and anxiety.

In particular, Chapter four analyzes the place learning abilities in a group of patients affected by panic disorder with agoraphobia using a virtual space. Results show that healthy subjects rapidly learned to locate the invisible target and consistently returned to it, while patients behaved in two different ways: some of them were as good as controls in place learning, while some others were unable to orientate themselves in the environment. Further analyses showed that age and duration of illness are critical factors that influence the place learning abilities. This study shows that a virtual space can be a very useful ecological instrument to detect and measure slight neuropsychological differences that could not be noticed in real life contexts, possibly providing new insight into the mechanisms of panic.

In Chapter five we described an explorative study that evaluates the effectiveness of a relaxation training supported by the use of technology (virtual reality and portable mp3 players) compared with relaxation based on imagination in a sample of female obese inpatients affected by emotional eating. The main results of this study is that a short relaxation training (12 sessions for 3 weeks) improves the patients' perceived self-efficacy producing a positive effect on their eating behavior and long-term weight loss. Thus, the lack of significant differences between the virtual reality and the imaginative condition suggests that VR may play a significant role in providing relaxation only if it is well technically and graphically arranged. Perhaps, for relaxation training purposes, providing immersion by a headmounted display with an embedded tracker device is not feasible, while the graphical realism and the interaction with the environment are critical factors that make the experience effective.

Chapter six and seven are about two experimental studies in which virtual reality has been used as an alternative medical treatment to reduce anxiety in minor surgical operations. In both studies we used a small, portable and immersive VR system to induce relaxation in patients who underwent minor surgical procedures. In one of the two studies we tested it on a sample of high-civilized people living in Mexico City, while in the other one we investigated the effect of the exposure to VR in a very primitive culture 
analyzing the role played by the cultural and technological background of the users in their emotional responses to technology. The "core affect" model of emotion developed by James Russell helped us to interpret the results: VR significantly modified the core affect in all subjects (as shown by the significant reduction of arousal), but the final emotional response produced by this change was influenced by the attribution process. The civilized inhabitants of Mexico City, who were able to attribute the reduced arousal to the VR experience, reported a significant reduction in their selfreported level of anxiety, while primitive people showed a reduction in their physiological reactions, but not in their perceived anxiety.

Differently form the previous chapters including experimental data, Chapter eight is a perspective study in which we discuss the role played by the threedimensional (3-D) virtual worlds in eHealth applications, addressing some potential advantages and issues related to the use of this emerging medium in clinical practice. The chapter describes the development and implementation of a form of tailored immersive e-therapy called $p$-health whose key factor is interreality, that is the creation of a hybrid personalized augmented experience merging physical and virtual worlds.

Finally, Chapter nine provides a general discussion that integrates the results of the different chapters. Limitations as well as possible risks and contraindications associated to the virtual approach are also presented. Furthermore, some suggestions for future research are proposed. 

Samenvatting 

Virtual Reality (VR) is een geavanceerde vorm van human-computerinterface waarmee gebruikers kunnen communiceren met de computer en worden ingebed in een gegenereerde omgeving in een naturalistische manier. Ongeveer twintig jaar geleden zijn psychologen clincal VR gaan gebruiken voor de behandeling van verschillende psychische stoornissen om zo te profiteren van de geboden mogelijkheid die de therapeuten hebben om in een volledig gecontroleerde omgeving patiënten onder directe toezicht bloot te stellen aan angstige stimuli. Dit proefschrift richt zich op een aantal zeer recente experimentele studies gericht op de VR-onderzoek naar de kenmerken van een klinische hulpmiddel voor de behandeling van stress en angst.

Hoofdstuk een geeft een algemene inleiding over VR: de technologische componenten worden in detail beschreven te samen met een korte geschiedenis van haar toepassingen in de klinische praktijk. De grondgedachte van het gebruik van VR in de gezondheidszorg wordt toegelicht en de voordelen van het gebruik daarvan voor de behandeling van angst worden beschreven.

In de daarop volgende twee hoofdstukken worden de psychologische aspecten in een virtuele ervaring besproken. In het bijzonder hoofdstuk twee introduceert het concept van aanwezigheid; een psychologische constructie die verwijst naar de illusie van anwezigheid in een (gesimuleerde) plaats wanneer men ook daadwerkelijk aanwezig is in een andere (fysieke) plaats. Aanwezigheid is een belangrijk kenmerk van een virtuele ervaring en wordt gegenereerd door verschillende technologische, cognitieve en emotionele factoren. In deze eerste studie hebben we getest hoe we de virtuele beleving kunnen optimaliseren en het gevoel van aanwezigheid van het manipuleren van een aantal van deze factoren te vergroten. We hebben concreet getest of een immersieve technologie en / of een zinvolle verhalende context van invloed is op gebruikers gevoel van aanwezigheid met een meer meeslepende ervaring, dan een niet-meeslepende en niet-gecontextualiseerd virtuele ruimte. Het resultaat was - zoals verwacht - dat beide blootstellingen en het verhaal belangrijk zijn om een effectieve virtual reality ervaring te creëren, waarbij elk anders bij dragen tot het vergroten van het gevoel van aanwezigheid. Immersion verhoogt de plaats illusie, terwijl het verhaal bij draagt om zo een emotionele respons te genereren en om persoons' gevoel van innerlijke aanwezigheid te versterken. Hoofdstuk drie is gericht op het experimenteel verifiëren van de theoretische gedachte achter de' virtuele anpak ", dat wil zeggen dat de reële en virtuele 
vorderingen een vergelijkbare emotionele reactie veroorzaken bij de gebruikers. Het blootstellen van twee soorten patiënten die lijden aan eetstoornissen door het eten van echt voedsel, vituele voeding en foto's van voedsel, resulteerden in het feit dat de virtuele blootstelling emotionele reacties ontlokken, welke vergelijkbaar zijn met een reële blootstelling en dat het gevoel van aanwezigheid veroorzaakt door VR onderdompeling, de virtuele ervaring meer ecologische, en bijgevolg efficiënter is dan het statische beeld van emotionele responsen in de mens. Het belang van deze studie vloeit voort uit het feit dat het de eerste poging binnen de gehele VR literatuur is in het experimenteel vergelijken van de effecten door middel van echte en virtuele blootstelling.

Vanaf hoofdstuk vier tot zeven beschrijven we vier studies waarin de VR is gebruikt om de angst van verschillende psychische stoornissen gerelateerd aan stress te evalueren en te behandelen. Met name hoofdstuk vier analyseerd de 'place learning vaardigheden' in een groep patiënten met paniekstoornissen met agorafobie met behulp van een virtuele ruimte. Resultaten tonen aan dat gezonde personen snel leerden om het onzichtbare object te lokaliseren en consequent naar teruggekeerden, terwijl de patiënten zich op twee verschillende manieren gedroegen: sommigen van hen waren goed in het controleren van place learning, terwijl andere niet in staat waren om zich te oriënteren in de omgeving. Verdere analyses hebben aangetoond dat leeftijd en duur van de ziekte kritische factoren zijn die de place learning vaardigheden beïnvloeden. Deze studie toont aan dat een virtuele ruimte een zeer nuttig ecologisch instrument kan zijn bij het detecteren en meten lichte neuropsychologische verschillen welke anders niet zou worden opgemerkt in 'real life contexts' en welke eventueel nieuwe inzichten in de mechanismen van paniek geeft.

In hoofdstuk vijf evalueren en beschrijven we een verkennende studie die de doeltreffendheid van een ontspanningstraining, ondersteund door het gebruik van de technologie (virtual reality en draagbare mp3-spelers) in vergelijking tot een ontspanning welke gebaseerd is op de verbeelding van zwaarlijvige vrouwelijke patiënten die lijden aan 'emotioneel eten'. De belangrijkste resultaten van deze studie zijn dat bij een korte ontspanningsoefening training (12 sessies in 3 weken), de patiënten zelf de positieve effecten van hun eetgedrag ervaren en het op de lange termijn resulteerd in gewichtsverlies. Dus het ontbreken van significante verschillen tussen de virtuele realiteit en de inbeeldings-situatie suggereerd dat de VR een belangrijke rol kan spelen bij creëren van ontspanning, alleen als het technisch en grafisch goed geregeld is. Misschien, voor 
ontspanningstraining doeleinden waarbij door middel van een onderdompeling met een head-mounted display middels een ingebedde tracker apparaat het gewenste effect niet haalbaar is, kunnen het grafische realisme en de interactie met de omgeving kritische factoren zijn die de ervaring effectief maken.

Hoofdstuk zes en zeven gaan over twee experimentele studies waarbij de virtual reality is gebruikt als alternatief voor een medische behandeling, om zo de angst bij kleine chirurgische operaties te verminderen. In beide studies gebruikten we een klein, draagbaar VR-systeem om ontspanning te induceren bij patiënten bij wie een kleine chirurgische ingreep heeft plaats gevonden. In een van de twee studies, welke getest is op de wat 'meerbeschaafde' mensen in Mexico City, terwijl we in de andere studie onderzocht hebben wat het effect is van blootstelling aan VR bij een zeer primitieve cultuur, waarbij we analyseerden welke rol de culturele en technologische achtergrond van de gebruikers speelt in hun emotionele reacties op technologie. Het 'core effect' model van emotie, ontwikkeld door Russell James heeft ons geholpen met de resultaten: VR heeft het 'core effect' aanzienlijk veranderd op alle onderwerpen (zoals blijkt uit de aanzienlijke vermindering van de opwinding), maar de uiteindelijke emotionele reactie, bewerkstelligd door deze verandering werd beïnvloed door het toekennings proces. De beschaafde inwoners van Mexico City, die in staat waren de verminderde opwinding toe te schrijven aan de VRervaring, rapporteerde een aanzienlijke verlaging van hun niveau van angst, terwijl de primitieve mensen een vermindering van hun fysiologische reacties lieten zien maar niet in hun vermeende angst.

Anders dan de voorgaande hoofdstukken, met inbegrip van de experimentele gegevens, beschrijft hoofdstuk acht een perspectieve studie waarin we ingaan op de rol welke gespeeld wordt door de drie-dimensionale (3-D) virtuele werelden in de eHealth-applicaties in samenhang met het een aantal potentiële voordelen en problemen gerelateerd aan het gebruik van dit opkomende medium in de klinische praktijk. Het hoofdstuk beschrijft de ontwikkeling en implementatie van een vorm van een op maat gemaakte immersieve e-therapie, p-gezondheidszorg genaamd, waarvan de belangrijkste factor interrealiteit is; de creatie van hybride persoonlijke ervaringen aangevuld met fuserende fysieke en virtuele werelden.

Ten slotte, voorziet hoofdstuk negen in een algemene discussie over de resultaten van de verschillende hoofdstukken. Beperkingen als ook mogelijke risico's en contra-indicaties verbonden aan de virtuele aanpak 
worden gepresenteerd. Voorts worden enkele suggesties voor toekomstig onderzoek voorgesteld. 


\section{Dankwoord}



My parents and a lot of friends of mine considered my Ph.D. as a "neverending story". But finally I've done it! One of the reasons of their distrust was that, in the last year, every time I came to Maastricht my Promotor, Professor Eric Griez, sent me back telling me: "Alessandra, you miss one more paper to conclude your thesis!" It was a hard work, but at the end of this story I'm really grateful to him who has sustained me spending many hours discussing about protocols and results on a topic that, at least at the beginning, was not so familiar for him. I hope he has enjoyed virtual reality!

I want to thank Koen Schruers, who accepted to be my Co-promotor, and all the members of the Reading Committee, who carefully read and judged this thesis.

Thanks to Brenda Wiederhold, one of the first psychologists who introduced the use of virtual reality for the treatment of anxiety, but also a good friend of mine. I'm sure she knows how glad I am to have an American scientist in the Reading Committee of my Ph.D., and I'm sure she is glad too because this makes her more European!

Thanks to Leni, the perfect secretary, who, at the beginning of this story, told me: "You have to finish your Ph.D. before my retirement". She has helped me a lot, especially in the last months, and I'm really grateful to her. Leni, now you are free to retire!

Thanks to Lies, who tried to teach me Dutch...unfortunately with no results (but I'm completely sure it was my fault!)

Motje, Annemie and Thijs: thank you very much for your great hospitality. Every time I come to your house I feel at home. That's great! I'll never forget the long relaxing and funny walks in the fields together with Motje... I've spent a really good time with all of you!

And now, after more than 200 pages in English, some words in Italian.

Un grazie a Giampaolo Perna che un giorno mi disse: "E' ora che tu vada a Maastricht a fare un'esperienza internazionale". Ora posso dirgli che è stata un'ottima idea. 
E grazie a Giuseppe Riva che mi ha introdotto all'affascinante mondo della realtà virtuale, e che mi ha fatto capire quanto sia importante scrivere, scrivere, scrivere... E questa tesi ne è la dimostrazione.

Grazie a Laura che ha ascoltato per intere serate davanti a Sushi e Philadelphia Maki le mie ansie per questo dottorato che sembrava non finire mai. Non ha mai smesso di dirmi che ce l'avrei fatta, e aveva ragione.

E un grazie particolare a Gabriella, che fino a ieri non c'era, ma che oggi mi ha fatto capire, grazie alla sua "laboriosità, generosità e umanità", dove sicuramente voglio essere domani.

E infine, un grazie molto speciale a chi mi ha permesso di arrivare fino a qui, lasciando che seguissi le mie inclinazioni e le mie passioni e che facessi ciò che mi piace davvero, senza mai farmi sentire il peso di una scelta professionale spesso difficile e tortuosa. Grazie Mamma e Papà!

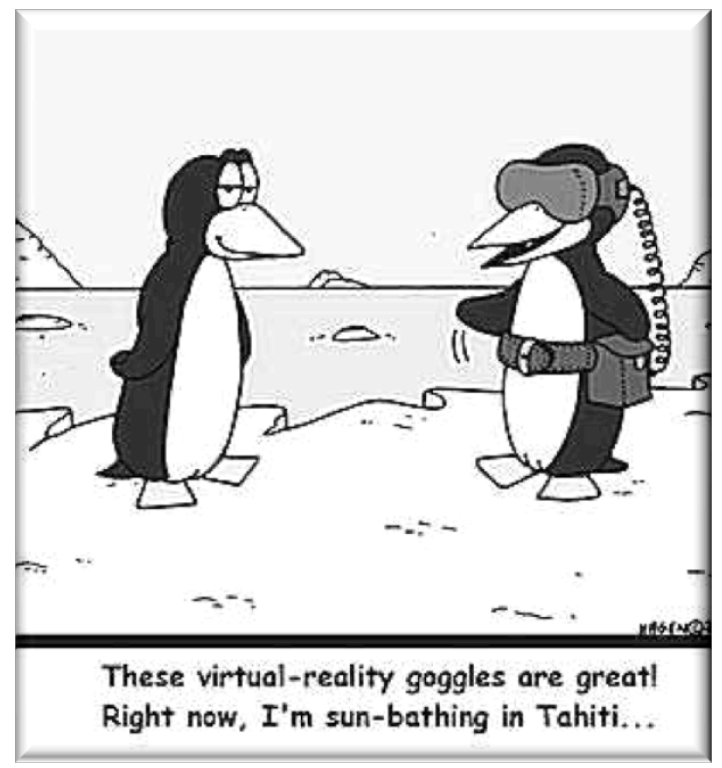




\section{Publications}





\section{Papers}

1. Carlesimo GA, Turriziani P, Paulesu E, Gorini A, et al. Brain activity during intra- and cross-modal priming: new empirical data and review of the literature. Neuropsychologia 2004; 42(1):14-24.

2. Canessa N, Gorini A, Cappa SF, et al. The effect of social content on deductive reasoning: an fMRI study. Hum Brain Mapp 2005; Sep; 26(1):30-43.

3. Cavedini P, Gorini A, Bellodi L. Understanding obsessivecompulsive disorder: focus on decision making. Neuropsychol Rev 2006; Mar; 16(1):3-15.

4. Cavedini P, Zorzi C, Bassi T, Gorini A, et al. Decision-making functioning as a predictor of treatment outcome in anorexia nervosa. Psychiatry Res 2006; Dec 7; 145(2-3):179-87.

5. Gorini A, Gaggioli A, Riva G. Virtual worlds, real healing. Science 2007; Dec 7; 318(5856):1549.

6. Gorini A, Gaggioli A, Vigna C, Riva G. A second life for eHealth: prospects for the use of 3-D virtual worlds in clinical psychology. J Med Internet Res 2008; 10(3):e21.

7. Gorini A, Riva G. The potential of Virtual Reality as anxiety management tool: a randomized controlled study in a sample of patients affected by Generalized Anxiety Disorder. Trials 2008; 9:25.

8. Gorini A, Riva G. Virtual reality in anxiety disorders: the past and the future. Expert Rev Neurother 2008; Feb; 8(2):215-33.

9. Manzoni GM, Gorini A, Preziosa A, et al. New technologies and relaxation: an explorative study on obese patients with emotional eating. Journal of Cybertherapy and Rehabilitation 2008; 1(2):18292.

10. Perani D, Garibotto V, Gorini A, et al. In vivo PET study of 5HT(2A) serotonin and $\mathrm{D}(2)$ dopamine dysfunction in drug-naive obsessive-compulsive disorder. Neuroimage 2008; Aug 1; 42(1):306-14.

11. Gorini A, Mosso JL, Mosso D, et al. Emotional Response to Virtual Reality Exposure across Different Cultures: The Role of the 
Attribution Process. Cyberpsychol Behav 2009; Nov 3.

12. Manzoni GM, Pagnini F, Gorini A, et al. Can relaxation training reduce emotional eating in women with obesity? An exploratory study with 3 months of follow-up. J Am Diet Assoc 2009; Aug; 109(8):1427-32.

13. Mosso JL, Gorini A, De La Cerda G, et al. Virtual reality on mobile phones to reduce anxiety in outpatient surgery. Stud Health Technol Inform 2009; 142:195-200.

14. Repetto C, Gorini A, Algeri D, Vigna C, Gaggioli A, Riva G. The use of biofeedback in clinical virtual reality: the intrepid project. Stud Health Technol Inform 2009; 144:128-32.

15. Repetto C, Gorini A, Vigna C, Algeri D, Pallavicini F, Riva G. The use of biofeedback in clinical virtual reality: the INTREPID project. J Vis Exp 2009; (33).

16. Riva G, Carelli L, Gaggioli A, Gorini A, et al. NeuroVR 1.5 in Practice: Actual Clinical Applications of the Open Source VR System. Stud Health Technol Inform 2009; 144:57-60.

17. Riva G, Carelli L, Gaggioli A, Gorini A, et al. NeuroVR 1.5 - a free virtual reality platform for the assessment and treatment in clinical psychology and neuroscience. Stud Health Technol Inform 2009; 142:268-70.

18. Riva G, Gorini A. CyberEurope. Cyberpsychol Behav 2009; Dec; 12(6):773.

19. Riva G, Gorini A, Gaggioli A. The Intrepid project - biosensorenhanced virtual therapy for the treatment of generalized anxiety disorders. Stud Health Technol Inform 2009; 142:271-6.

20. Garibotto V, Scifo P, Gorini A, et al. Disorganization of anatomical connectivity in Obsessive Compulsive Disorder: a multi-parameter Diffusion Tensor Imaging study in a subpopulation of patients. Neurobiol Dis 2010; Nov 11; 37(2):468-76.

21. Gorini A, Pallavicini F, Algeri D, Repetto C, Gaggioli A, Riva G. Virtual reality in the treatment of generalized anxiety disorders. Stud Health Technol Inform 2010; 154:39-43.

22. Riva G, Raspelli S, Algeri D, Pallavicini F., Gorini A., et al. Interreality in Practice: Bridging Virtual and Real Worlds in the 
Treatment of Posttraumatic Stress Disorders. Cyberpsychol Behav 2010; Feb 13(1): 55-65.

23. Gorini A, Griez E, Petrova A, Riva G. Assessment of the emotional responses produced by exposure to real food, virtual food and photographs of food in patients affected by eating disorders. Annals of General Psychiatry 2010; Jul 5; 9(1): 30.

24. Gorini A, Schruers K, Riva G, Griez E. Nonhomogeneous results in place learning among panic disorder patients with agoraphobia. Psychiatry Res 2010; May 28.

25. Gorini A, Capideville CS, De Leo G, Mantovani F, Riva G. The role of immersion and narrative in mediated presence: the virtual hospital experience. Cyberpsychol Behav 2010; Jul 22.

\section{Books}

1. Erzegovesi S, Gorini A. (2004) Bella come sei. Affrontare la bulimia. Milano: San Paolo. Translated in: Polish (2004), Spanish (2005).

2. Gorini A. (2006) La memoria: una, nessuna, centomila. Milan: San Paolo.

3. Canessa N, Gorini A (eds). Manuale di Scienze Cognitive. Torino: Einaudi; 2008.

\section{Book Chapters}

1. Cavedini P, Gorini A, Bassi T, Zorzi C, Bellodi L. (2005) A neuropsychological investigation of decisional processes in obsessive-compulsive spectrum disorders Progress in ObsessiveCompulsive Disorder Research: Nova Science Publishers.

2. Bellodi L, Gorini A. (2005) La memoria In: Bellodi L (ed). Manuale di Psicopatologia: Masson. 
3. Bellodi L, Gorini A. (2005) L'attenzione. In: Bellodi L (ed). Manuale di Psicopatologia: Masson.

4. Bellodi L, Gorini A. (2005) La coscienza. In: Bellodi L (ed). Manuale di Psicopatologia: Masson.

5. Tundo A, Bellodi L, Gorini A, Mauri M. (2006) I disturbi di personalità. In: Cassano GB, Tundo A (eds). Psicopatologia e Clinica Psichiatrica: Utet.

6. Gorini A, Gaggioli A, Riva G. (2010) Virtual Reality as an Experiential Tool: The Role of Virtual Worlds in Psychological Interventions. In: Mohammed S, Flaidhi J (eds). Ubiquitous Health and Medical Informatics.

7. Masiero M, Gorini A, (in press) Il burn-out negli operatori sanitari. In: Pravettoni G, Vago G (eds). Medical Decision Making. Decidere in Ambito Sanitario: McGraw-Hill: Milano 


\section{Curriculum Vitae}



Alessandra Gorini was born on June 151976 in Milan, Italy. In 1996 she started her studies in experimental psychology at the Università Vita e Salute San Raffaele in Milan, where she obtained her candidate diploma in Psychology Cum Laude in 2001. For this degree she performed a research internship at the department of neuroimaging concerning functional MRI experiments on healthy volunteers and psychiatric patients. It was during this internship that she gained interest in performing research in the field of Neuroscience.

In December 2004 she obtained a Master in Clinical Neuropsychology at the Università di Padova, Italy.

After some research and clinical experience in the field of Affective Neuroscience in Italy, she moved to Maastricht, where she obtained the European Certificate in Affective Neuroscience in July 2005 and the Master in Affective Neuroscience in July 2006 at the School of Mental Health and Neurosciences (MHeNS, division Mental Health) of the Maastricht University under the supervision of Professor Eric Griez.

In 2007 she moved back to Milan, where she worked as young researcher on a European Project regarding the use of virtual reality for the treatment of anxiety disorders at the Istituto Auxologico Italiano. Her growing interest in the field of virtual reality and new technologies has produced a significant number of publications on International peer reviewed journals.

She is actually collaborating with IRIDe (Centro Interdipartimentale di Ricerca e Intervento sui Processi Decisionali), at the Università degli Studi di Milano, where she investigates cognitive and emotional factors involved in the decision-making processes. 